\title{
Guía de Práctica Clínica sobre el abordaje de síntomas vasomotores y vaginales asociados a la menopausia y la postmenopausia
}





\section{Guía de Práctica Clínica sobre el abordaje de síntomas vasomotores y vaginales asociados a la menopausia y la postmenopausia}

GUÍAS DE PRÁCTICA CLÍNICA EN EL SNS

MINISTERIO DE SANIDAD, SERVICIOS SOCIALES E IGUALDAD

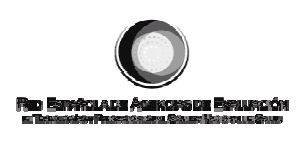


Esta Guía de Práctica Clínica es una ayuda para la toma de decisiones en la atención sanitaria. No es de obligado cumplimiento ni sustituye el juicio clínico del personal sanitario.

Año de edición: 2017

Edita: Ministerio de Sanidad, Servicios Sociales e Igualdad.

Edita: Agencia de Evaluación de Tecnologías Sanitarias de Andalucía. Consejería de Salud - JUNTA DE ANDALUch

NIPO: Ministerio de Sanidad, Servicios Sociales e Igualdad 860-11-183-7. Ministerio de Ciencia e Innovación 477-11-056-4 ISBN: 978-84-694-8236-0 
Este documento se ha realizado al amparo del convenio de colaboración suscrito por el Instituto de Salud Carlos III, organismo autónomo del Ministerio de Economía y Competitividad, y la Consejería de Salud de la Junta de Andalucía, en el marco del desarrollo de actividades de la Red Española de Agencias de Evaluación de Tecnologías Sanitarias y Prestaciones del SNS, financiadas por el Ministerio de Sanidad, Servicios Sociales e Igualdad.

\section{Esta gula debe citarse:}

Grupo de trabajo de la Guía de Práctica Clínica sobre el abordaje de síntomas vasomotores y vaginales asociados a la menopausia y la postmenopausia. Guía de Práctica Clínica sobre el abordaje de síntomas vasomotores y vaginales asociados a la menopausia y la postmenopausia. Ministerio de Sanidad, Servicios Sociales e Igualdad. Agencia de Evaluación de Tecnologías Sanitarias de Andalucía (AETSA); 2017. Guías de Práctica Clínica en el SNS. 



\section{Índice}

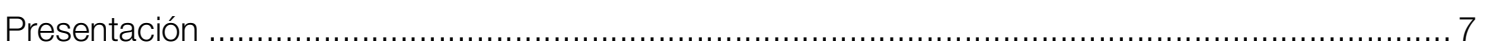

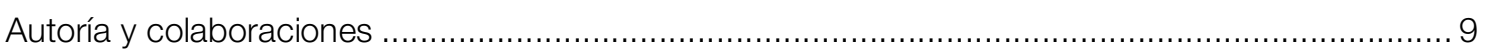

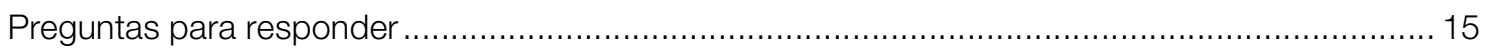

Método GRADE: Calidad de la evidencia y fuerza de la recomendación ....................................... 17

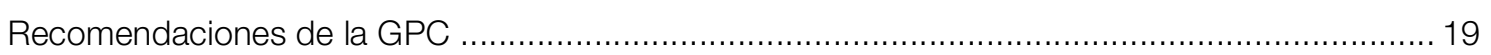

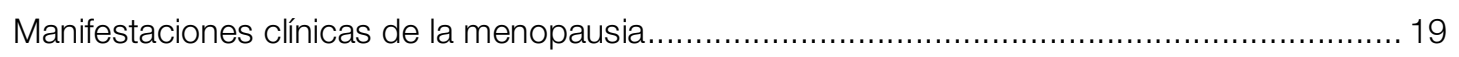

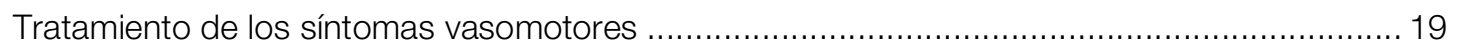

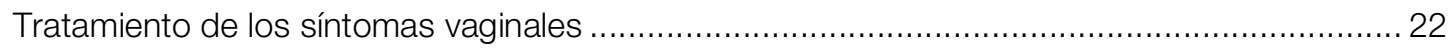

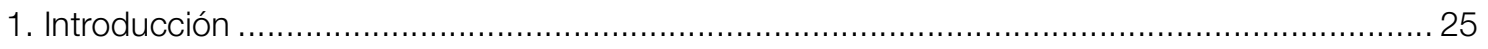

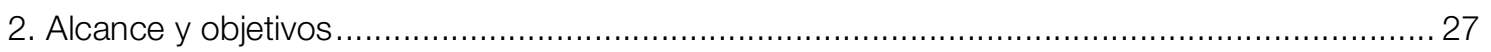

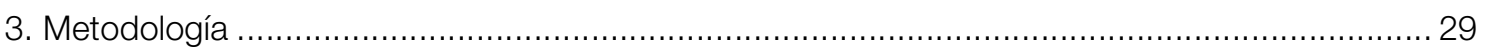

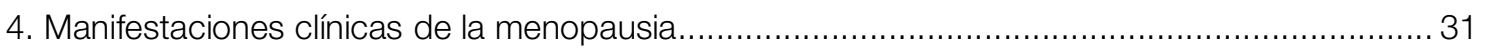

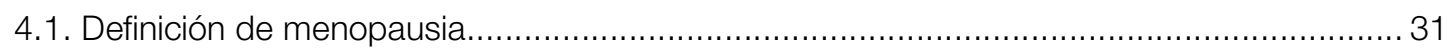

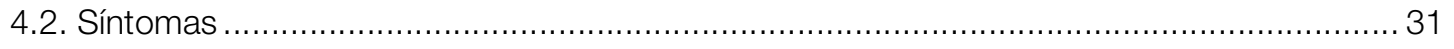

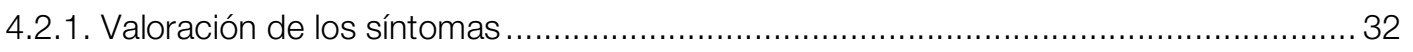

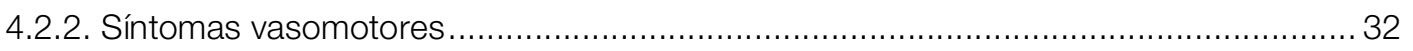

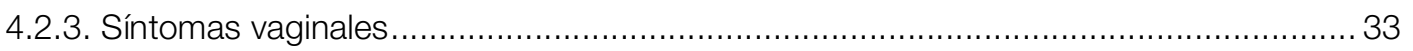

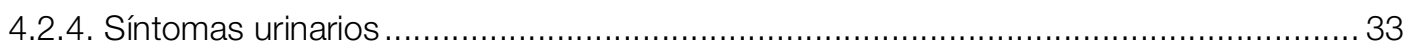

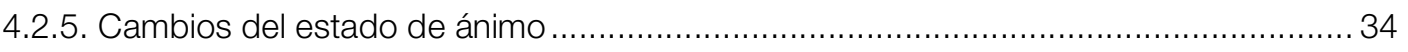

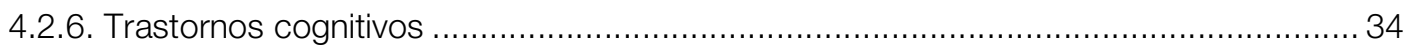

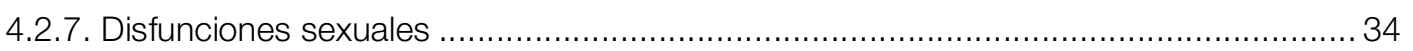

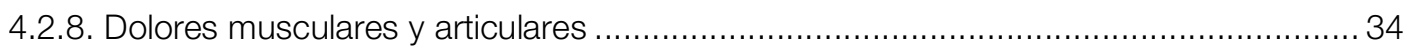

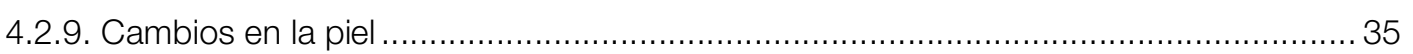

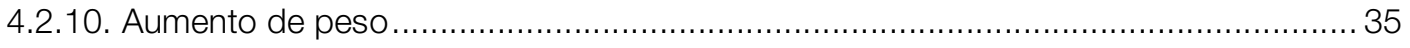

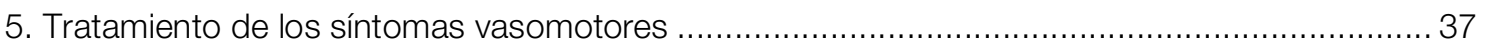

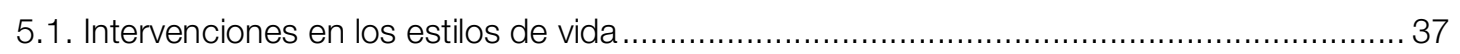

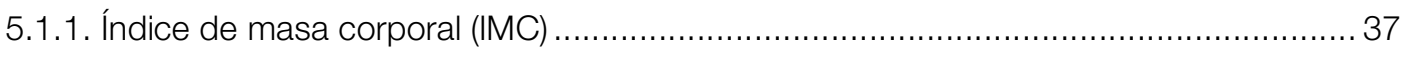

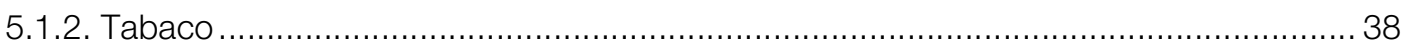

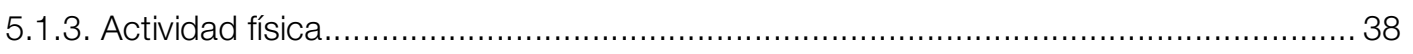

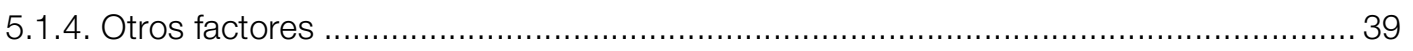

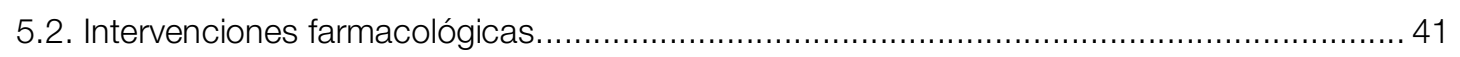

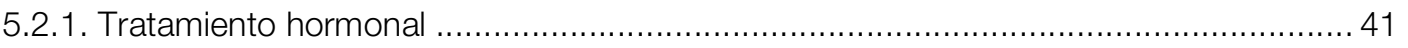

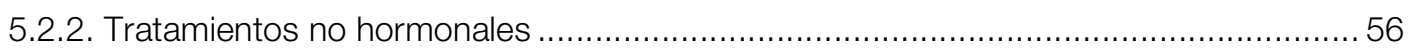

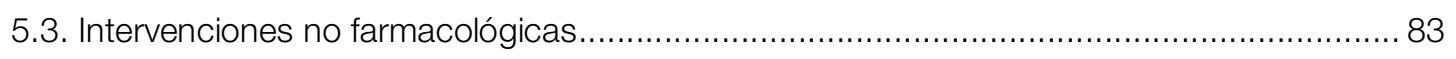

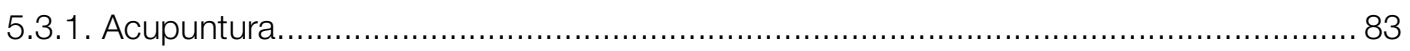

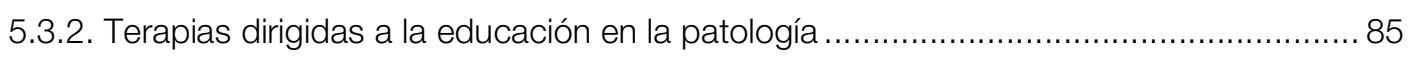

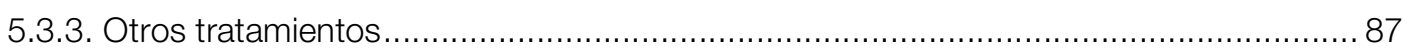




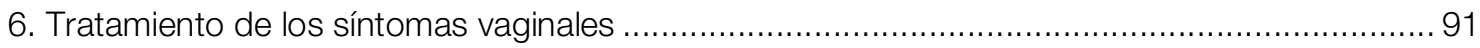

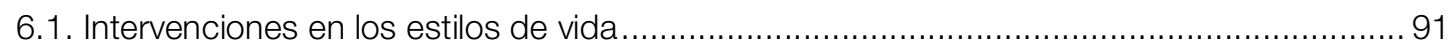

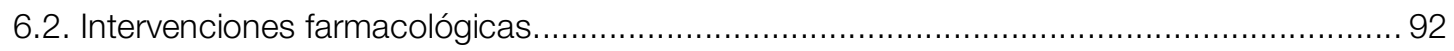

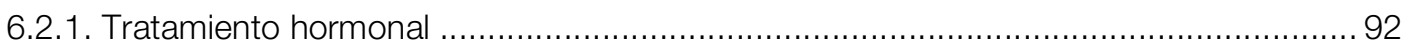

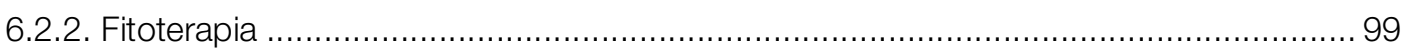

6.2.3. Moduladores selectivos de los receptores estrogénicos (SERM) …......................... 103

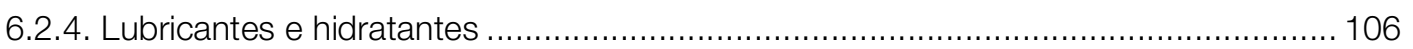

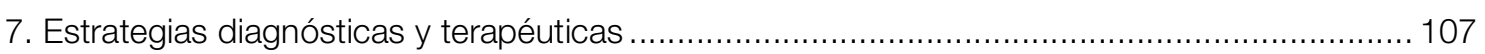

Algoritmo 1. Mujeres con síntomas vasomotores .......................................................... 107

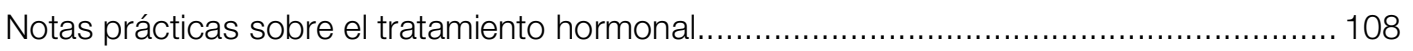

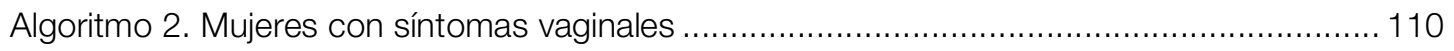

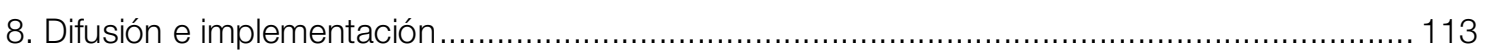

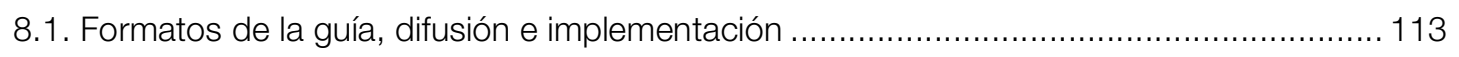

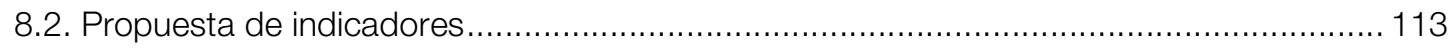

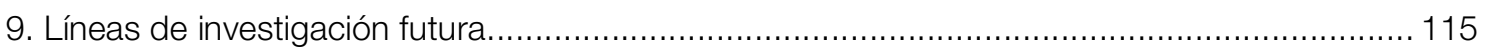

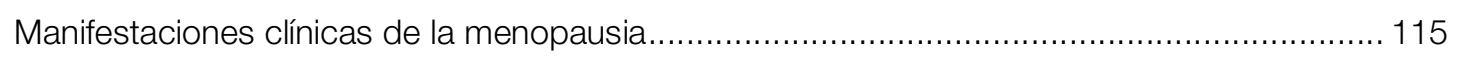

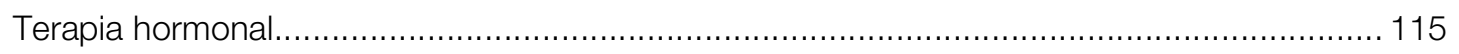

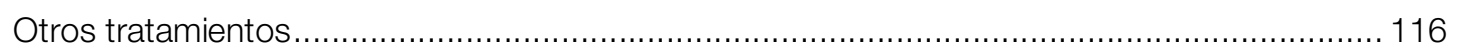

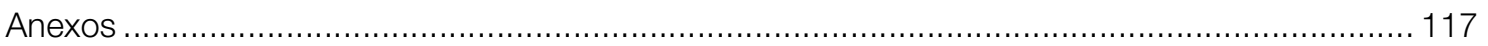

Anexo 1. Evaluación de la calidad de la evidencia y la graduación de las recomendaciones

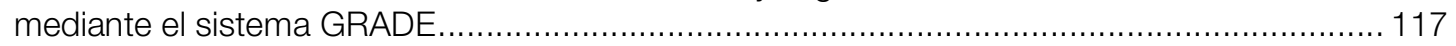

A) Clasificación de la importancia relativa de las variables de resultado ............................ 117

B) Evaluación de la calidad de la evidencia científica ........................................................ 118

C) Graduación de la fuerza de las recomendaciones ........................................................... 119

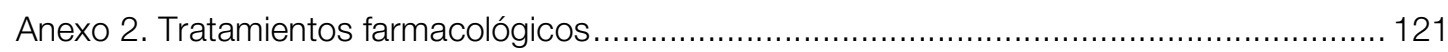

Anexo 3. Principales guías de práctica clínica y otros recursos de utilidad ........................... 123

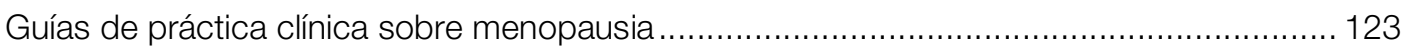

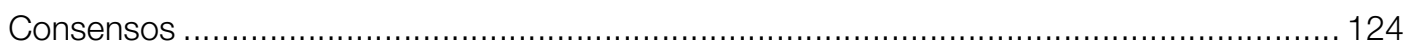

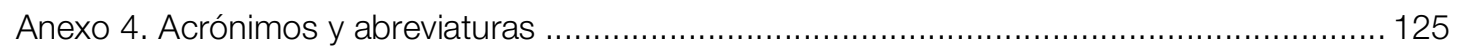

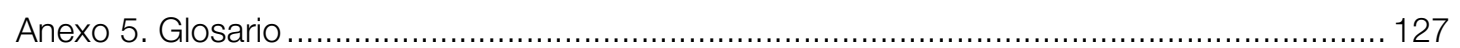

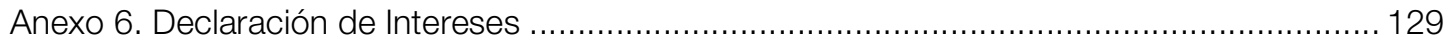

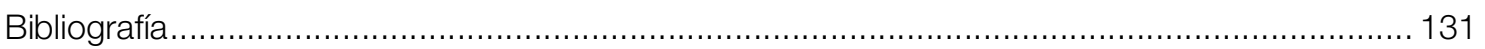




\section{Presentación}

Documentar la variabilidad de la práctica clínica, analizar sus causas y adoptar estrategias orientadas a eliminarla, han demostrado ser iniciativas que fomentan la toma de decisiones efectivas y seguras, centradas en las y los pacientes, por parte de los profesionales sanitarios. Entre dichas estrategias destaca la elaboración de Guías de Práctica Clínica (GPC), «conjunto de recomendaciones basadas en una revisión sistemática de la evidencia y en la evaluación de los riesgos y beneficios de las diferentes alternativas, con el objetivo de optimizar la atención sanitaria a los pacientes».

Entre las prioridades del Ministerio de Sanidad, Servicios Sociales e Igualdad, se encuentra consolidar la elaboración de GPC, coordinada desde GuíaSalud, en el marco de la Red Española de Agencias de Evaluación de Tecnologías Sanitarias y Prestaciones del Sistema Nacional de Salud (SNS). Es en este contexto en el que se enmarca la presente Guía de Práctica Clínica sobre el abordaje de síntomas vasomotores y vaginales asociados a la menopausia y la postmenopausia.

La menopausia es una etapa fisiológica en la vida de las mujeres que viene determinada por la disminución y cese definitivo de la actividad ovárica. Los cambios hormonales que se producen como consecuencia pueden asociarse a la aparición de síntomas vasomotores y vaginales que con el tiempo, normalmente, van perdiendo intensidad y terminan desapareciendo. No obstante, en algunos casos, estos síntomas pueden persistir más de cinco años y, en ocasiones, afectar la calidad de vida de las mujeres.

El objetivo de esta GPC es proporcionar una síntesis de la evidencia científica disponible y recomendaciones clínicas elaboradas en base a éstas sobre las posibles intervenciones, sus beneficios y sus riesgos -ya sean farmacológicas o no farmacológicas-, dirigidas a minimizar el impacto de la llegada de la menopausia en la calidad de vida de la mujer.

Este conocimiento servirá a los profesionales de la salud tanto como herramienta de ayuda a la toma decisiones sobre la mejor alternativa a recomendar en cada caso, como para poder trasmitir a la mujer información objetiva y rigurosa que facilite su participación en la decisión final.

La GPC responde a preguntas clínicas concernientes a epidemiología de los posibles síntomas asociados a la menopausia, los beneficios de los estilos de vida saludable, los beneficios y riesgos de tratamientos farmacológicos y de determinadas terapias considerada medicina alternativa.

Se dirige tanto a profesionales de los equipos de atención primaria como a los equipos de centros o servicios de atención a la salud sexual y reproductiva, y tiene como fin adicional disminuir la variabilidad de práctica clínica y facilitar la coordinación entre el ámbito de Atención Primaria y Hospitalaria.

Esta guía es el resultado de la labor desarrollada por el grupo de trabajo constituido por profesionales sanitarios pertenecientes a distintas especialidades y representantes de varias Sociedades Científicas.

Elena Andradas Aragonés. Directora General de Salud Pública, Calidad e Innovación. 



\section{Autoría y colaboraciones}

\section{Grupo de trabajo de la GPC sobre la menopausia y la postmenopausia}

Francesc Baró-Mariné. Médico especialista en ginecología y obstetricia, Hospital Universitario Vall d'Hebron, Barcelona. Profesor Asociado de la Cátedra de Ginecología y Obstetricia de la UAB

(Barcelona).

Emilia Bailon-Muñoz. Médica especialista en Medicina Familiar y Comunitaria. Centro de Salud Albaycin. Vocal de la Comision Nacional de la Especialidad de Medicina Familiar y Comunitaria, Ministerio de Sanidad y Consumo (Granada).

Jacobo Cambil-Martín. Profesor de Enfermería Familiar y Comunitaria. Departamento de Enfermería de la Universidad de Granada (Granada).

Joaquín Calaf-Alsina. Médico especialista en ginecología y obstetricia, Hospital de la Santa Creu i Sant Pau, Barcelona.

M. a Jesús Cancelo-Hidalgo. Médica especialista en ginecología y obstetricia, Hospital Universitario de Guadalajara, Profesora asociada Universidad de Alcalá (Madrid).

M. Jesús Cornellana-Puigarnau. Médica especialista en ginecología y obstetricia, Hospital del Mar. Presidenta de la Sección de Menopausia de la Societat Catalana d'Obstetrícia i Ginecologia

(Barcelona).

M. José Iglesias-Piñeiro. Médica especialista en medicina familiar y comunitaria, Centro de Salud Vicente Soldevilla. Grupo de la mujer del PAPPS. Sociedad Española de Medicina de Familia y Comunitaria

(Madrid).

Victoria Jiménez-Espínola. Farmacéutica, técnico de evaluación de Centro Andaluz de Documentación e Información de Medicamentos (CADIME) (Granada).

M. a Dolores Juliá-Mollá. Médica especialista en ginecología y obstetricia, Servicio de Ginecología y Reproducción, Hospital Universitario La Fe. Universidad de Valencia (Valencia). 
Laia Llort-i-Samsó. Médica especialista en medicina familiar y comunitaria, ABS Les Borges Blanques, Sap Segrià-Garrigues

(Lleida).

Alberto López-García-Franco. Médico especialista en medicina familiar y comunitaria, Centro de Salud Mendiguchía Carriche, Leganés, Madrid. Coordinador del Grupo de la Mujer del PAPPS

(Madrid).

Juliana Ester Martín-López. Médica de familia y técnico de investigación, Agencia de Evaluación de Tecnologías Sanitarias de Andalucía (AETSA) (Sevilla).

Teresa Molina-López. Directora de la Agencia de Evaluación de Tecnologías Sanitarias de Andalucía (AETSA) (Sevilla).

Núria Nadal-Braqué. Médica especialista en medicina familiar y comunitaria, SAP Segrià-Garrigues (Lleida).

Maria Teresa Nieto-Rodríguez. Farmacéutica, técnico de evaluación de Centro Andaluz de Documentación e Información de Medicamentos (CADIME) (Granada).

Sonia Peláez-Moya. Jefa de servicio del observatorio de salud de las mujeres, Ministerio de Salud, Servicios Sociales e Igualdad

(Madrid).

Esther Redondo-Margüello. Médica especialista en medicina familiar y comunitaria, Centro de Salud Internacional, Madrid Salud. Grupo de Actividades preventivas y de la mujer de SEMERGEN

(Madrid).

María de las Nieves Respaldiza-Salas. Médica especialista en inmunología y técnico de investigación, Agencia de Evaluación de Tecnologías Sanitarias de Andalucía (AETSA) (Sevilla).

Mercedes Ricote-Belinchon. Médica especialista en medicina familiar y comunitaria. SERMAS Centro de Salud Mar Báltico. Vocal de docencia de la Junta Autonómica de SEMERGEN-MADRID

(Madrid).

Dolores Ruiz-Berdún. Matrona y documentalista, profesora ayudante doctora en Historia de la Ciencia en la Universidad de Alcalá, Alcalá de Henares (Madrid).

M. ${ }^{a}$ Ángeles Ruiz-Algora. Enfermera gestora de casos del Distrito Sevilla (Sevilla). 
M. ${ }^{a}$ Dolores Saavedra-Llobregat. Enfermera de gestión comunitaria Dto. Alicante/Hospital General, Profesora Universidad de Alicante (Alicante).

Juan José Sagarzazu-Goenaga. Médico especialista en medicina familiar y comunitaria, miembro del Grupo de MBE de Osatzen, Centro de Salud de Oñati (Gipuzkoa).

Rafael Sánchez-Borrego. Médico especialista en ginecología y obstetricia, Clínica DIATROS de Atención a la Mujer. Presidente de la Asociación Española para el Estudio de la Menopausia.

(Barcelona).

Carmen Suárez-Alemán. Farmacéutica de atención primaria, Agencia de Evaluación de Tecnologías Sanitarias de Andalucía (AETSA) (Sevilla).

\section{Coordinación}

Carmen Suárez-Alemán. Farmacéutica de atención primaria, Agencia de Evaluación de Tecnologías Sanitarias de Andalucía (AETSA) (Sevilla).

Juliana Ester Martín-López. Médica de familia y técnico de investigación, Agencia de Evaluación de Tecnologías Sanitarias de Andalucía (AETSA) (Sevilla).

Teresa Molina-López. Directora de la Agencia de Evaluación de Tecnologías Sanitarias de Andalucía (AETSA) (Sevilla).

\section{Revisión externa}

Paloma Casado-Pérez. Médica especialista en medicina familiar y comunitaria, Servicio Madrileño de Salud (Madrid).

Gema Coira-Fernández. Médica especialista en medicina familiar y comunitaria, Coordinadora del Grupo de Trabajo de Atención a la Mujer de la Sociedad Española de Medicina Familiar y Comunitaria (SEMFyC), Servicio de Salud del Principado de Asturias (SESPA)

(Asturias).

Arritxu Etxeberria-Agirre. Farmacéutica de Atención Primaria, Centro de Salud de Hernani, Osakidetza, Sociedad Española de Farmacéuticos de Atención Primaria (SEFAP) (País Vasco). 
Carlos Fernandez-Oropesa. Farmacéutico en atención primaria y especialista en farmacia hospitalaria, Área de gestión sanitaria Nordeste de Granada, Servicio Andaluz de Salud (Granada).

M. ' José Gimeno-Tortajada. Médica especialista en medicina familiar y comunitaria, Centro de salud de San Marcelino. Valencia.

Grupo de osteoporosis y locomotor de Semergen (Valencia).

Misericordia Guinot-Gasull. Médica especialista en ginecología y obstetricia, Responsable de la Unidad de Menopausia. Hospital de la Santa Creu i Sant Pau (Barcelona).

Rosa María López-Rodríguez. Coordinadora de programas. Subdirección General de Calidad e Innovación. Dirección General de Salud Pública, Calidad e Innovación. Ministerio de Sanidad, Servicios Sociales e Igualdad (Madrid).

Soledad Marquez-Calderón. Médico especialista en medicina preventiva, Consejería de Igualdad, Salud y Políticas Sociales. Servicio de Calidad y Procesos (Sevilla).

Pilar Miranda-Serrano. Médica especialista en Obstetricia y Ginecologia, Desarrollos Sanitarios Madrileños, Sociedad española de ginecología y Obstetricia (SEGO) (Madrid).

Paula Quesada-Luiáñez. Enfermera especialista en obstetricia y ginecología, Servicio Andaluz de Salud (Sevilla). 


\section{Agradecimientos}

Pablo Alonso-Coello. Médico especialista en medicina familiar y comunitaria, Centro Cochrane Iberoamericano, Hospital de la Santa Creu i Sant Pau y CIBER de Epidemiología y Salud Pública

(CIBERESP) (Barcelona).

Laura Martínez-García. Médica especialista en medicina preventiva y salud pública, Centro Cochrane Iberoamericano, Hospital de la Santa Creu i Sant Pau (Barcelona).

David Rigau-Comas. Médico especialista en farmacología clínica, Centro Cochrane Iberoamericano, Hospital de la Santa Creu i Sant Pau (Barcelona).

Ivan Solà-Arnau. Área de documentación, Centro Cochrane Iberoamericano, Hospital de la Santa Creu i Sant Pau (Barcelona).

Sera Tort-Donada. Médica especialista en medicina familiar y comunitaria, Centro Cochrane Iberoamericano, Hospital de la Santa Creu i Sant Pau (Barcelona).

Carme Valls-Llobet. Médica directora del Programa Mujer, Salud y Calidad de Vida del CAPS (Barcelona).

\section{Sociedades y Asociaciones colaboradoras}

Sociedad Española de Farmacéuticos de Atención Primaria (SEFAP). Sociedad Española de Medicina Familiar y Comunitaria (SEMFYC).

Sociedad Española de Ginecología y Obstetricia (SEGO).

Declaración de intereses: Todos los miembros del grupo de trabajo, así como las personas que han participado en la colaboración experta y en la revisión externa, han realizado la declaración de intereses que se presenta en el Anexo 6. 



\section{Preguntas para responder}

\section{DEFINICIÓN}

1. ¿Qué síntomas presentan una asociación causal con la disminución de estrógenos en la mujer durante la postmenopausia?

2. ¿Cuál es la mejor manera de valorar los síntomas vasomotores? ¿Hay alguna escala diagnóstica?

3. ¿Qué otras causas de sofocos existen?

\section{TRATAMIENTO DE LOS SINTOMAS VASOMOTORES}

4. En mujeres con síntomas vasomotores durante la peri y postmenopausia, ¿cuál es la eficacia y la seguridad de la modificación en los estilos de vida?

5. En mujeres con síntomas vasomotores durante la peri y postmenopausia, ¿cuál es la eficacia y la seguridad de la terapia hormonal con estrógenos y estrógenos combinados con gestágenos?

6. En mujeres con síntomas vasomotores durante la peri y postmenopausia, ¿cuál es la eficacia y la seguridad de la terapia hormonal con gestágenos?

7. En mujeres con síntomas vasomotores durante la peri y postmenopausia, ¿cuál es la eficacia y la seguridad de la terapia hormonal con andrógenos?

8. En mujeres con síntomas vasomotores durante la peri y postmenopausia, ¿cuál es la eficacia y la seguridad de la terapia hormonal con tibolona?

9. En mujeres con síntomas vasomotores durante la peri y postmenopausia, ¿cuál es la eficacia y la seguridad de los antidepresivos?

10. En mujeres con síntomas vasomotores durante la peri y postmenopausia, ¿cuál es la eficacia y la seguridad de otros fármacos como la gabapentina, metildopa y clonidina?

11. En mujeres con síntomas vasomotores durante la peri y postmenopausia, ¿cuál es la eficacia y la seguridad de la fitoterapia?

12. En mujeres con síntomas vasomotores durante la peri y postmenopausia, ¿cuál es la eficacia y la seguridad de los tratamientos no farmacológicos?

13. En mujeres con síntomas vasomotores durante la peri y postmenopausia, ¿cuál es la eficacia y la seguridad de otras terapias alternativas? 


\section{TRATAMIENTO DE LOS SÍNTOMAS VAGINALES}

14. En mujeres con síntomas vaginales durante la peri y postmenopausia, ¿cuál es la eficacia y la seguridad de la modificación de los estilos de vida?

15. En mujeres con síntomas vaginales durante la peri y postmenopausia, ¿cuál es la eficacia y la seguridad de los estrógenos?

16. En mujeres con síntomas vaginales durante la peri y postmenopausia, ¿cuál es la eficacia y la seguridad de los gestágenos?

17. En mujeres con síntomas vaginales durante la peri y postmenopausia, ¿cuál es la eficacia y la seguridad de la tibolona?

18. En mujeres con síntomas vaginales durante la peri y postmenopausia, ¿cuál es la eficacia y la seguridad de la fitoterapia?

19. En mujeres con síntomas vaginales durante la peri y postmenopausia, ¿cuál es la eficacia y la seguridad de los moduladores selectivos de los receptores estrogénicos (SERM)?

20. En mujeres con síntomas vaginales durante la peri y postmenopausia, ¿cuál es la eficacia y la seguridad de los lubricantes e hidratantes vaginales? 


\section{Método GRADE: Calidad de la evidencia y fuerza de la recomendación}

\begin{tabular}{|c|c|c|c|}
\hline \multicolumn{4}{|c|}{ Clasificación de la calidad de la evidencia en el sistema GRADE } \\
\hline $\begin{array}{c}\text { Calidad de } \\
\text { la evidencia } \\
\text { científica }\end{array}$ & $\begin{array}{l}\text { Diseño del } \\
\text { estudio }\end{array}$ & Disminuir la calidad si & Aumentar la calidad si \\
\hline Alta & ECA & \multirow{6}{*}{$\begin{array}{l}\text { Limitación en el diseño: } \\
\text { Importante (-1) } \\
\text { Muy importante (-2) } \\
\text { Inconsistencia (-1) } \\
\text { Evidencia directa: } \\
\text { Alguna (-1) incertidumbre } \\
\text { Gran (-2) incertidumbre } \\
\text { acerca de que la evidencia } \\
\text { sea directa } \\
\text { Datos imprecisos (-1) } \\
\text { Sesgo de notificación: } \\
\text { Alta probabilidad de (-1) }\end{array}$} & \multirow{6}{*}{$\begin{array}{l}\text { Asociación: } \\
\text { - Evidencia científica de una fuerte } \\
\text { asociación (RR }>20<0,5 \\
\text { basado en estudios } \\
\text { observacionales sin factores de } \\
\text { confusión) (+1) } \\
\text { - Evidencia científica de una muy } \\
\text { fuerte asociación (RR > } 50<0,2 \\
\text { basado en estudios sin } \\
\text { posibilidad de sesgos) (+2) } \\
\text { Gradiente dosis respuesta (+1) } \\
\text { Todos los posibles factores } \\
\text { confusores podrían haber } \\
\text { reducido el efecto observado (+1) }\end{array}$} \\
\hline Moderada & & & \\
\hline Baja & $\begin{array}{c}\text { Estudios } \\
\text { observacionales }\end{array}$ & & \\
\hline Muy baja & $\begin{array}{l}\text { Otros tipos de } \\
\text { diseño }\end{array}$ & & \\
\hline & & & \\
\hline & & & \\
\hline
\end{tabular}

\begin{tabular}{|c|c|c|}
\hline \multicolumn{3}{|c|}{ Implicaciones de la fuerza de recomendación en el sistema GRADE } \\
\hline \multicolumn{3}{|c|}{ Implicaciones de una recomendación fuerte } \\
\hline Pacientes & $\begin{array}{c}\text { Profesionales asistenciales / } \\
\text { Personal Clínico }\end{array}$ & $\begin{array}{c}\text { Profesionales } \\
\text { de Gestión / Planificación }\end{array}$ \\
\hline $\begin{array}{l}\text { La inmensa mayoría de las } \\
\text { mujeres estarían de acuerdo con } \\
\text { la acción recomendada y } \\
\text { únicamente una pequeña parte } \\
\text { no lo estarían }\end{array}$ & $\begin{array}{l}\text { La mayoría de las mujeres } \\
\text { deberían recibir la intervención } \\
\text { recomendada }\end{array}$ & $\begin{array}{l}\text { La recomendación puede ser } \\
\text { adoptada como política } \\
\text { sanitaria en la mayoría de las } \\
\text { situaciones }\end{array}$ \\
\hline \multicolumn{3}{|c|}{ Implicaciones de una recomendación débil } \\
\hline Pacientes & $\begin{array}{c}\text { Profesionales asistenciales / } \\
\text { Personal Clínico }\end{array}$ & $\begin{array}{c}\text { Profesionales } \\
\text { de Gestión / Planificación }\end{array}$ \\
\hline $\begin{array}{l}\text { La mayoría de las mujeres } \\
\text { estarían de acuerdo con la } \\
\text { acción recomendada pero un } \\
\text { número importante de ellas no }\end{array}$ & $\begin{array}{l}\text { Reconoce que diferentes } \\
\text { opciones serán apropiadas } \\
\text { para diferentes mujeres y que el } \\
\text { médico tiene que ayudar a } \\
\text { cada mujer a llegar a la } \\
\text { decisión más consistente con } \\
\text { sus valores y preferencias }\end{array}$ & $\begin{array}{l}\text { Existe necesidad de un debate } \\
\text { importante y la participación de } \\
\text { los grupos de interés }\end{array}$ \\
\hline
\end{tabular}





\section{Recomendaciones de la GPC}

La evaluación de la calidad de la evidencia y la graduación de las recomendaciones se ha realizado mediante el sistema GRADE (Grading of Recommendations of Assessment Development and Evaluations) (ver Anexo 1). Las recomendaciones pueden ser fuertes, débiles o de buena práctica clínica (BPC). A continuación se incluyen las recomendaciones propuestas en esta GPC.

\section{Manifestaciones clínicas de la menopausia}

\begin{tabular}{|l|l|}
\hline \multirow{3}{*}{ BPC } & $\begin{array}{l}\text { Se sugiere informar a las mujeres de que la menopausia solo se ha } \\
\text { demostrado que se asocie a síntomas vasomotores y vaginales, y no a } \\
\text { otros síntomas que habitualmente se han creído asociados a la } \\
\text { menopausia como los síntomas urinarios, la ansiedad, los cambios de } \\
\text { humor, la irritabilidad y la depresión. }\end{array}$ \\
\hline
\end{tabular}

\section{Tratamiento de los síntomas vasomotores}

\section{Intervenciones en los estilos de vida}

\begin{tabular}{|c|l|}
\hline FUERTE & $\begin{array}{l}\text { En mujeres que presentan síntomas vasomotores durante el climaterio, en } \\
\text { la etapa de la perimenopausia y postmenopausia, se recomienda evitar la } \\
\text { obesidad, realizar ejercicio físico y evitar el consumo de tabaco para } \\
\text { reducir los síntomas vasomotores. }\end{array}$ \\
\hline DÉBIL & $\begin{array}{l}\text { En mujeres con síntomas vasomotores durante la peri y postmenopausia } \\
\text { se sugiere procurar un ambiente fresco, disminuir el consumo de cafeína, } \\
\text { bebidas calientes, alcohol y comidas picantes para no exacerbar la } \\
\text { sintomatología. }\end{array}$ \\
\hline
\end{tabular}




\section{Intervenciones farmacológicas}

Estrógenos solos y combinados con gestágenos

\begin{tabular}{|c|c|}
\hline FUERTE & $\begin{array}{l}\text { No se recomienda el tratamiento hormonal en mujeres con síntomas } \\
\text { vasomotores leves. }\end{array}$ \\
\hline DÉBIL & $\begin{array}{l}\text { En mujeres durante la etapa de la peri y postmenopausia con síntomas } \\
\text { vasomotores intensos que afecten de manera importante su calidad de } \\
\text { vida, se sugiere ofrecer la posibilidad de realizar tratamiento hormonal, } \\
\text { informando con claridad sobre su efectividad para reducir los síntomas y } \\
\text { sobre todos los riesgos asociados, facilitando que sea la mujer quien } \\
\text { decida con esta información si iniciar el tratamiento. }\end{array}$ \\
\hline FUERTE & $\begin{array}{l}\text { La terapia hormonal está contraindicada en mujeres con antecedentes de } \\
\text { cáncer de mama o de endometrio, enfermedad coronaria, tromboembolismo, } \\
\text { ictus, hepatopatía activa, enfermedad pancreática y de vesícula biliar o bien } \\
\text { con alto riesgo de padecer estas enfermedades. }\end{array}$ \\
\hline FUERTE & $\begin{array}{l}\text { En las mujeres que conservan el útero se recomienda añadir gestágenos al } \\
\text { tratamiento con estrógenos. }\end{array}$ \\
\hline BPC & $\begin{array}{l}\text { El tratamiento hormonal se mantendrá mientras persista la } \\
\text { sintomatología y durante el menor tiempo posible, limitándose a un } \\
\text { periodo máximo de un año. Posteriormente, el tratamiento debería ser } \\
\text { individualizado. Se recomienda iniciar el tratamiento hormonal con dosis } \\
\text { bajas e incrementarlas periódicamente hasta la remisión o mejora } \\
\text { importante de la sintomatología. Una vez alcanzado el efecto deseado } \\
\text { puede intentarse un proceso inverso de disminución gradual de la dosis y } \\
\text { en caso de recidiva de la sintomatología, volver a la última dosis eficaz. }\end{array}$ \\
\hline BPC & $\begin{array}{l}\text { La elección de la vía y el tipo de tratamiento puede realizarla la mujer } \\
\text { debidamente informada, teniendo en cuenta que, utilizando dosis bajas o } \\
\text { ultrabajas, las pautas secuenciales raramente inducen sangrado por } \\
\text { deprivación. }\end{array}$ \\
\hline BPC & $\begin{array}{l}\text { El/la profesional puede adaptar las dosis a las necesidades individuales de } \\
\text { la mujer (administrar el tratamiento a intervalos más largos, dividir } \\
\text { comprimidos o parches). }\end{array}$ \\
\hline BPC & $\begin{array}{l}\text { Se recomienda implicar a la mujer en la decisión de iniciar el tratamiento } \\
\text { hormonal. }\end{array}$ \\
\hline
\end{tabular}




\begin{tabular}{|c|l|}
\hline DÉBIL & $\begin{array}{l}\text { En mujeres en la etapa de la peri y postmenopausia con contraindicaciones } \\
\text { en el uso de estrógenos y síntomas vasomotores intensos, se sugiere valorar } \\
\text { la utilización de los gestágenos por vía oral o intramuscular, individualizando } \\
\text { el balance beneficio/riesgo para el tratamiento de los síntomas vasomotores. }\end{array}$ \\
\hline
\end{tabular}

\section{Andrógenos}

\begin{tabular}{l|l} 
DÉBIL & $\begin{array}{l}\text { Se sugiere no utilizar el tratamiento con andrógenos para tratar los síntomas } \\
\text { vasomotores en mujeres durante la etapa de la peri y postmenopausia. }\end{array}$
\end{tabular}

\section{Tibolona}

\begin{tabular}{|c|l|}
\hline DÉBIL & $\begin{array}{l}\text { En mujeres en la etapa de la postmenopausia con síntomas vasomotores } \\
\text { intensos que afecten su calidad de vida y en las que el tratamiento } \\
\text { hormonal con estrógenos no haya sido efectivo o no se tolere, se puede } \\
\text { valorar iniciar el tratamiento con tibolona, teniendo en cuenta el } \\
\text { riesgo/beneficio de esta intervención. }\end{array}$ \\
\hline BPC & $\begin{array}{l}\text { En las mujeres en las que sea necesario iniciar el tratamiento con tibolona } \\
\text { durante la etapa de la postmenopausia, se sugiere administrarla después } \\
\text { del primer año de menopausia. Si el tratamiento se inicia antes, aumentan } \\
\text { las posibilidades de sangrado vaginal irregular o manchado. }\end{array}$ \\
\hline
\end{tabular}

Antidepresivos y otros fármacos

\begin{tabular}{|c|l|}
\hline DÉBIL & $\begin{array}{l}\text { En mujeres durante la etapa de la peri y postmenopausia con síntomas } \\
\text { vasomotores moderados o intensos que empeoren su calidad de vida y en } \\
\text { las que esté contraindicado el tratamiento hormonal o que no deseen } \\
\text { tomarlo, y en las que las modificaciones del estilo de vida resultan } \\
\text { ineficaces, se sugiere valorar el tratamiento con antidepresivos (paroxetina } \\
\text { o desvenlafaxina) o gabapentina* }\end{array}$ \\
\hline DÉBIL & $\begin{array}{l}\text { En las mujeres durante la peri o postmenopausia con síntomas } \\
\text { vasomotores moderados o intensos que afecten a su calidad de vida, en las } \\
\text { que el tratamiento hormonal, los antidepresivos y la gabapentina están } \\
\text { contraindicados o no deseen tomarlos, se puede valorar la administración } \\
\text { de clonidina. }\end{array}$ \\
\hline
\end{tabular}

* En el momento de la publicación de esta guía, la paroxetina, desvenlafaxina y gabapentina no tiene autorizada en España esta indicación. Según el Real Decreto 1015/2009 que regula la disponibilidad de medicamentos en situaciones especiales, los medicamentos utilizados fuera de sus indicaciones aprobadas requieren informar al paciente y/o a su familia, solicitar el consentimiento y reflejarlo en la historia clínica. 


\begin{tabular}{|c|l|}
\hline DÉBIL & $\begin{array}{l}\text { En mujeres en etapa peri y postmenopáusica que no realizan tratamiento } \\
\text { hormonal (o que éste está contraindicado) y que presentan síntomas } \\
\text { vasomotores que afectan a su calidad de vida, se sugiere valorar el tratamiento } \\
\text { con fitoestrógenos (extractos naturales o sintéticos de soja, concentrados de } \\
\text { genisteína* o suplementos dietéticos de soja). En la elección del preparado, } \\
\text { debe considerarse y conocerse la composición así como la dosis empleada. }\end{array}$ \\
\hline DÉBIL & $\begin{array}{l}\text { Se sugiere no utilizar el tratamiento con otros fitoestrógenos, Cimifuga } \\
\text { Racemosa y otros tratamientos de hierbas medicinales en mujeres con } \\
\text { síntomas vasomotores en etapa peri y postmenopáusica. }\end{array}$ \\
\hline FUERTE & $\begin{array}{l}\text { No se debe administrar Cimifuga Racemosa a aquellas mujeres con } \\
\text { enfermedad hepática conocida o en tratamiento con fármacos hepatotóxicos. }\end{array}$ \\
\hline
\end{tabular}

* Los concentrados de genisteína no se encuentran comercializados en España.

\section{Intervenciones no farmacológicas}

\begin{tabular}{|c|l|}
\hline DÉBIL & $\begin{array}{l}\text { En mujeres con síntomas vasomotores durante la etapa de la peri y } \\
\text { postmenopausia, se sugiere valorar como tratamientos complementarios } \\
\text { la acupuntura o las intervenciones educativas (terapia cognitivo- } \\
\text { conductual y la reducción del estrés basada en la atención plena) sobre } \\
\text { los síntomas vasomotores. }\end{array}$ \\
\hline
\end{tabular}

\section{Tratamiento de los síntomas vaginales}

\section{Intervenciones en los estilos de vida}

\begin{tabular}{|l|l|}
\hline FUERTE & $\begin{array}{l}\text { En mujeres durante la peri y postmenopausia se recomienda evitar la } \\
\text { obesidad y realizar ejercicio físico para prevenir la aparición de } \\
\text { síntomas vaginales. }\end{array}$ \\
\hline
\end{tabular}

\section{Intervenciones farmacológicas}

Estrógenos

\begin{tabular}{|l|l|}
\hline FUERTE & $\begin{array}{l}\text { En mujeres en la etapa de postmenopausia con síntomas vaginales } \\
\text { moderados o intensos, se recomienda ofrecer la posibilidad de realizar } \\
\text { tratamiento con estrógenos vaginales en forma de crema, óvulos o anillo } \\
\text { vaginal, informando con claridad sobre su efectividad para reducir los } \\
\text { síntomas y sobre todos los riesgos asociados, facilitando que sea la mujer } \\
\text { quien decida si iniciar el tratamiento con esta información. }\end{array}$ \\
\hline
\end{tabular}




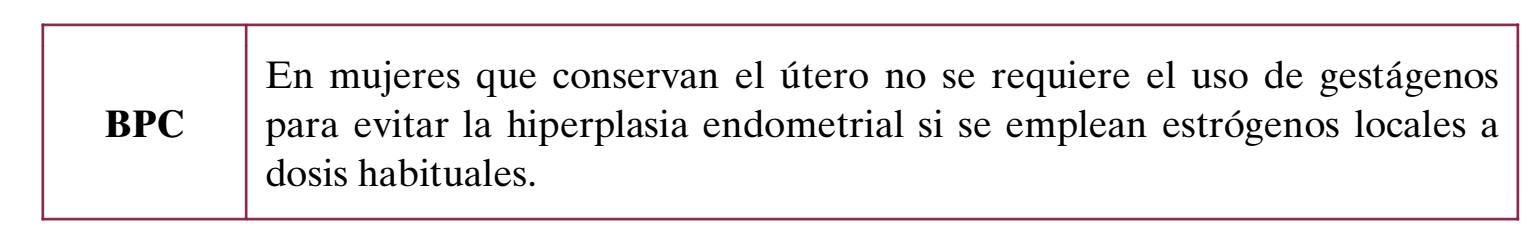

\section{Tibolona}

\begin{tabular}{|l|l|}
\hline DÉBIL & $\begin{array}{l}\text { En mujeres en la etapa de la postmenopausia, con síntomas vaginales } \\
\text { moderados o intensos y las que el tratamiento hormonal con estrógenos } \\
\text { no haya sido efectivo o no se tolere, se sugiere valorar el tratamiento con } \\
\text { tibolona para los síntomas vaginales, teniendo en cuenta el riesgo / } \\
\text { beneficio de esta intervención }\end{array}$ \\
\hline
\end{tabular}

Fitoterapia

\begin{tabular}{|c|l|}
\hline DÉBIL & $\begin{array}{l}\text { Se sugiere no utilizar el tratamiento con fitoestrógenos, Cimifuga } \\
\text { Racemosa o Pueraria mirifica para tratar los síntomas vaginales en las } \\
\text { mujeres durante la peri y postmenopausia. }\end{array}$ \\
\hline
\end{tabular}

Moduladores selectivos de los receptores estrogénicos (SERM)

\begin{tabular}{|c|l|}
\hline DÉBIL & $\begin{array}{l}\text { Se sugiere no utilizar el tratamiento con moduladores selectivos de los } \\
\text { receptores estrogénicos (ospemifeno, bafedoxifeno y raloxifeno) para } \\
\text { mejorar los síntomas vaginales en mujeres durante las etapas peri y } \\
\text { postmenopáusica. }\end{array}$ \\
\hline
\end{tabular}

\section{Lubricantes e hidratantes}

DÉBIL

En mujeres con síntomas de dispareunia durante la peri y postmenopausia se sugiere valorar el uso de lubricantes e hidratantes vaginales.

a Ver Recomendación de BPC referida a Tibolona en "Tratamiento de los síntomas vasomoteres". 



\section{Introducción}

La menopausia es el cese definitivo de la menstruación por pérdida de la actividad folicular ovárica. Es una etapa fisiológica de la vida y como tal no puede ser considerada una enfermedad. Los síntomas asociados a la menopausia que pueden presentarse en esta etapa de la vida de la mujer tienen una importancia relativa ya que, aunque son prevalentes, tienen una mortalidad nula y una morbilidad variable ${ }^{1}$.

La menopausia es la consecuencia de la depleción de folículos en el ovario. Por esta razón su edad media de aparición se mantiene constante en el tiempo independientemente de las mejorías en el estado de salud o las expectativas de vida de la mujer. En algunas ocasiones, las pérdidas significativas de salud debidas a intervenciones médicas (quimioterapia, radioterapia), a la cirugía, a ciertos factores de riesgo (como el tabaquismo) o a diversas patologías, pueden adelantar su aparición respecto a la edad esperada ${ }^{2}$. Algunas mujeres tienen una transición asintomática hacia el climaterio*, pero la mayoría experimentan síntomas que pueden comenzar desde antes del cese de la menstruación y prolongarse en el tiempo, incluso hasta la senectud.

Debido al aumento de la esperanza de vida en España, en los próximos años se incrementará considerablemente el número de mujeres que estén en este periodo de la vida, donde paralelamente, tanto para hombres como para mujeres, el perfil epidemiológico se caracteriza por una mayor prevalencia de patologías crónicas.

A pesar de que los cambios biológicos asociados con la menopausia son universales, existen variaciones notables en la experiencia subjetiva entre las mujeres, entre diferentes culturas y en la manera en que estos afectan a su calidad de vida ${ }^{3}$. Los estudios muestran que las diferentes actitudes, creencias y percepciones hacia la menopausia se explican por la combinación de factores personales, culturales y ambientales ${ }^{4}$. La interacción entre la genética, que determinará el número de ovocitos presentes en el momento del nacimiento, el entorno, influido por aspectos culturales y socioeconómicos que determinaran el nivel cultural, el tipo de dieta, el consumo o no de tabaco y la práctica de ejercicio, contribuirán a que se produzcan diferencias en la aparición y percepción de los síntomas entre las diferentes mujeres 5 .

La menopausia se ha visto implicada en el fenómeno de la medicalización de la vida, de forma que este proceso fisiológico se ha visto expuesto a la intervención desde el ámbito sanitario. Sin embargo, la vivencia de la menopausia varía enormemente dependiendo de las actitudes personales y de la consideración del entorno sobre el envejecimiento en general y sobre el papel que ocupa la mujer mayor en la sociedad ${ }^{6}$. Muchos síntomas se han asociado a los cambios hormonales que ocurren en la menopausia, pero los únicos con una base epidemiológica sólida y consistente son la atrofia urogenital y los trastornos vasomotores ${ }^{7,8}$. Otros síntomas comunes como cambios en el estado de ánimo, trastornos del sueño, incontinencia urinaria, cambios cognitivos,

*Ver definición en el Glosario. 
o las molestias somáticas, pueden ser secundarios a otras causas. El aumento de la morbilidad de ciertas enfermedades en esta etapa de la vida (hipertensión arterial, diabetes mellitus, etc) está más ligado al proceso en sí de envejecimiento y los hábitos de vida que con la menopausia ${ }^{1}$.

Hasta hace unos 30 años la menopausia había pasado desapercibida para el ámbito sanitario. Pero en consonancia con la evolución sociocultural de los países desarrollados (el aumento de la esperanza de vida, la búsqueda de una mejor calidad de vida, etc.) el abordaje de la menopausia se vio también influenciado por estos factores.

Debido a la evidencia de que los estrógenos podrían mejorar no sólo los síntomas más frecuentes y molestos del climaterio (sofocos, sequedad vaginal, etc.), a partir de los años 80 se comenzó a utilizar la terapia hormonal para tratar los síntomas clínicos asociados a la menopausia. Paralelamente se potenciaron los cambios en los estilos de vida y el autocuidado como un pilar básico en la salud en esta etapa de la vida. En el año 2002 el estudio WHI (Women's Health Initiative) mostró que el tratamiento con terapia hormonal presentaba más riesgos que beneficios. Debido a esto, disminuyó drásticamente la prescripción de la terapia hormonal drásticamente quedando reservada sólo para mujeres sintomáticas, a la mínima dosis eficaz y durante el menor tiempo posible ${ }^{9}$. Actualmente las estrategias más habituales para tratar los síntomas leves derivados de la menopausia son los tratamientos no farmacológicos y potenciar el cambio de estilo de vida a hábitos saludables. Pero en situaciones en los que los síntomas disminuyen mucho la calidad de vida, la terapia hormonal sigue siendo el tratamiento farmacológico más efectivo, individualizando su utilización en cada caso y sopesando siempre el balance beneficio-riesgo.

Esta Guía de Práctica Clínica (GPC) forma parte del plan de elaboración de GPC del Ministerio de Sanidad, Servicios Sociales e Igualdad ${ }^{10}$. El propósito del Programa de GPC en el Sistema Nacional de Salud (SNS) es promover la elaboración, adaptación y/o actualización de GPC basadas en la mejor evidencia científica disponible con una metodología homogénea y contrastada y difundir las GPC en el SNS. Esta guía pretende clarificar qué síntomas son derivados del déficit hormonal secundario a la pérdida de actividad folicular del ovario, y cuál es la eficacia y la seguridad de los tratamientos disponibles. De esta forma, se espera contribuir a la disminución de la variabilidad de la práctica clínica y a la mejora de la atención sanitaria, proporcionando el mejor cuidado para las mujeres en esta etapa de la vida. 


\section{Alcance y objetivos}

Esta GPC aborda la identificación y el tratamiento de los síntomas vasomotores y vaginales asociados con la menopausia.

La población diana de esta guía son las mujeres en las etapas de la peri y postmenopausia, ya sea con un inicio espontáneo de la misma o secundario a causas médicas (tratamientos iatrogénicos) o quirúrgicas. Se excluye explícitamente el tratamiento de la osteoporosis. Tampoco se tratará en esta guía el uso de la terapia hormonal en mujeres con demencia y/o problemas cardiovasculares, si bien estas patologías serán tenidas en cuenta por lo que respecta a efectos secundarios y seguridad de la terapia hormonal. Tampoco se considerarán los síntomas asociados con la menopausia en subpoblaciones especiales (como mujeres con cáncer de mama), las irregularidades del ciclo menstrual asociadas a la transición menopáusica, ni el tratamiento específico de la menopausia precoz, más allá del tratamiento de su sintomatología. En estos grupos poblacionales excluidos las recomendaciones de esta guía pueden no ser aplicables.

El objetivo de esta GPC es establecer un conjunto de recomendaciones basadas en la evidencia científica acerca de la atención de las mujeres que presentan síntomas asociados con la menopausia.

La guía va dirigida específicamente a profesionales médicos/as generales/de familia, ginecólogos, de enfermería y matronas que desarrollan su actividad en atención primaria y en atención especializada. Son también posibles destinatarios de la misma otros profesionales de la salud involucrados en el cuidado de la mujer, como fisioterapeutas, psicólogos, psiquiatras, trabajadores sociales, etc., así como las personas responsables de estrategias de salud y a los gestores. Asimismo, la guía va dirigida a las mujeres, especialmente a aquellas con síntomas asociados con la menopausia. Por último, esta guía puede servir al público en general con interés en la menopausia y postmenopausia. 



\section{Metodología}

La metodología empleada se recoge de forma detallada en el Manual Metodológico para la elaboración de Guías de Práctica Clínica del Sistema Nacional de Salud, disponible en http://www.guiasalud.es $/{ }^{10}$.

Etapas:

- Constitución del grupo elaborador de la guía, integrado por profesionales de atención primaria (medicina familiar y comunitaria, enfermería comunitaria, matronas) y especializada (en ginecología y obstetricia). Las personas expertas y profesionales fueron contactadas a través de las distintas Sociedades Científicas relacionadas con el tema de la GPC. El material para las usuarias fue supervisado por una asociación de mujeres (Asociación Mujeres y Salud de Villena [Alicante]).

- Formulación de preguntas clínicas siguiendo el formato PICO: Paciente, Intervención, Comparación y resultado (Outcome). En el anexo 2 figuran los principales tratamientos farmacológicos y alternativos abordados por esta guía.

- Búsqueda bibliográfica, priorizando la identificación de revisiones sistemáticas (RS) y otros documentos de síntesis crítica de literatura científica, como informes de evaluación de tecnologías sanitarias. Para ello, en una primera fase se realizó una búsqueda de otras GPC para comprobar qué RS consideraron para apoyar sus recomendaciones. Las principales GPC utilizadas como fuentes secundarias están incluidas en el anexo 3. Posteriormente, se identificaron RS adicionales a partir de la fecha de búsqueda de las GPC seleccionadas. En esta primera etapa se consultaron las siguientes bases de datos electrónicas:

- TRIP Database.

○ NHS National Library of Guidelines.

- AHRQ National Guideline Clearinghouse.

- Cochrane Database of Systematic Reviews (The Cochrane Library).

- Database of Abstracts of Reviews of Effects (DARE).

- Health Technology Assessment (HTA) Database.

- NHS Economic Evaluation Database (NHS EED).

○ MEDLINE (accedido mediante PubMed).

- EMBASE (accedido mediante Ovid).

Además, se consultaron las publicaciones de una serie de agencias de evaluación de tecnologías como el National Institute for Clinical Excellence (NICE) y agencias productoras de GPC como la Scottish Intercollegiate Guidelines Network (SIGN). 
- En una segunda fase, se realizó una búsqueda ampliada de estudios individuales para actualizar las RS relevantes y contestar a las preguntas de la GPC. Principalmente, se identificaron ensayos clínicos aleatorizados (ECA) y estudios observacionales respetando las estrategias de búsqueda originales de las RS relevantes. Cuando éstas no estuvieron disponibles, se diseñó una estrategia específica para cada una de las preguntas, añadiendo en cada caso filtros validados para la identificación de ECA y estudios observacionales. En esta fase se consultaron las siguientes bases de datos electrónicas: The Cochrane Central Register of Controlled Trials (CENTRAL) (The Cochrane Library), MEDLINE, EMBASE, Cumulative Index to Nursing and Allied Health Literature (CINAHL) (a las que se accedió mediante Ovid).

No se estableció ningún límite lingüístico para las búsquedas llevadas a cabo. Se llevaron a cabo búsquedas hasta marzo de 2014, aunque se identificaron estudios relevantes en las revistas biomédicas de mayor impacto durante todo el proceso de elaboración de la GPC.

La Guía de Práctica Clínica sobre la menopausia y postmenopausia del año 2004 ha constituido un documento de referencia para el desarrollo de la actual guía ${ }^{11}$.

- La evaluación de la calidad de la evidencia y la graduación de las recomendaciones se ha realizado mediante el sistema GRADE (Grading of Recommendations of Assessment Development and Evaluations) ${ }^{12}$ (ver Anexo 1).

- El texto ha sido revisado por un grupo multidisciplinar de revisores externos. La versión final del texto de la guía ha sido aprobado por el grupo de autoras y autores.

- En www.guiasalud.es se describe el proceso metodológico de la GPC (estrategias de búsquedas para cada pregunta clínica, tablas de calidad metodológica y tablas de síntesis de la evidencia).

- Está prevista una actualización de la guía cada tres años, o en un plazo de tiempo inferior si aparece nueva evidencia científica que pueda modificar algunas de las recomendaciones ofrecidas en esta guía. Las actualizaciones se realizarán sobre la versión electrónica de la guía ${ }^{\mathrm{b}}$.

Durante el proceso de revisión se detectó la publicación de una revisión sistemática elaborada por la Agency for Healthcare Research and Quality (AHRQ) ${ }^{\mathrm{c}}$ sobre la efectividad comparativa de los tratamientos usados para tratar los síntomas de la menopausia. No se encontraron discrepancias relevantes con la evidencia y recomendaciones recogidas por esta guía.

\footnotetext{
${ }^{\mathrm{b}}$ Disponible en la URL: http://www.guiasalud.es.

c Grant MD, Marbella A, Wang AT, Pines E, Hoag J, Bonnell C, Ziegler KM, Aronson N. Menopausal Symptoms: Comparative Effectiveness of Therapies. Comparative Effectiveness Review No. 147. AHRQ Publication No. 15-EHC005-EF. Rockville, MD: Agency for Healthcare Research and Quality; March 2015. www.effectivehealthcare.ahrq.gov/reports/final.cfm.
} 


\section{Manifestaciones clínicas de la menopausia}

\section{Preguntas para responder}

- ¿Qué síntomas presentan una asociación causal con la disminución de estrógenos en la mujer en la fase postmenopáusica?

- ¿Cuál es la mejor manera de valorar los síntomas asociados a la menopausia? ¿Hay alguna escala diagnóstica?

- ¿Qué otras causas de sofocos existen?

\subsection{Definición de menopausia}

La menopausia se define como el cese de la menstruación durante 12 meses consecutivos sin otra causa aparente. Su causa natural radica en la reducción de la reserva folicular hasta un nivel crítico estimado en unos 1.000 ovocitos. Esta situación lleva asociada modificaciones endocrinológicas, especialmente déficit de estradiol y aumento de FSH y LH ${ }^{1}$.

El periodo de transición desde la época fértil de la mujer hacia la menopausia, se caracteriza endocrinológicamente por la pérdida de la actividad ovárica, biológicamente por la pérdida de la fertilidad y clínicamente por la alteración de los ciclos menstruales y la aparición de síntomas vasomotores y urogenitales.

La edad de presentación de la menopausia natural se sitúa entre los 45 y 55 años, con una media alrededor de los 50 años, tanto en la población mundial ${ }^{13}$ como en nuestro entorno $^{14}$. La menopausia precoz hace referencia a aquella que ocurre antes de los 40 años ${ }^{1}$. La edad de aparición de la menopausia puede influir en el riesgo de sufrir ciertas enfermedades y en la mortalidad, observándose un ligero incremento de la misma en las mujeres con menopausia en edades jóvenes ${ }^{2}$. Algunos factores que se relacionan con la edad de aparición de la menopausia son: la menarquia temprana, el bajo índice de masa corporal (IMC) y el consumo de tabaco (que pueden adelantarla), así como la edad de la menopausia en la madre y hermanas ${ }^{15}$.

\subsection{Síntomas}

En la transición menopáusica y la postmenopausia pueden aparecer síntomas, que aunque son generalmente autolimitados, pueden causar molestias y comprometer la calidad de vida. Se dispone de algunos estudios observacionales que han encontrado asociaciones de algunos de estos síntomas con la menopausia, aunque no en todos ellos se ha podido establecer una asociación clara. En la mayoría de las mujeres estos síntomas 
se resuelven entre los seis meses y los cinco años, aunque en un $25 \%$ de los casos persisten más de cinco años y en un $10 \%$, más de diez años.

En cualquier caso, la experiencia de cada mujer puede estar modulada por múltiples factores: etnia y cultura, genética, estilo de vida, paridad, índice de masa corporal (IMC), actividad física, etc., de forma que las variaciones biológicas y culturales modulan o influencian la transición menopáusica ${ }^{5}$.

\subsubsection{Valoración de los síntomas}

En la transición menopáusica y la postmenopausia pueden aparecer síntomas como los trastornos vasomotores y la atrofia vaginal que pueden causar malestar y empeorar la calidad de vida de las mujeres. Se han desarrollado varios métodos (listas o cuestionarios) para evaluar estos síntomas y otros que se cree están asociados con la menopausia, como los cambios en el estado de ánimo y/o en la calidad de vida, función sexual y síntomas somáticos. Algunos ejemplos son la escala climatérica de Greene ${ }^{16}$, el Índice de Kupperman ${ }^{17,18}$ (IK) y el cuestionario de calidad de vida en relación con la menopausia ${ }^{19}$. Estos cuestionarios, al evaluar distintos síntomas con distintos métodos, no permiten comparaciones entre estudios y a menudo no ajustan sus resultados por variables como la edad, patología previa o el uso de terapia hormonal. El más conocido, el índice de Kupperman, omite síntomas como la sequedad vaginal y presenta términos mal definidos, por lo que no se considera un método válido para evaluar los síntomas menopáusicos en la práctica clínica diaria. En España existe la Escala Cervantes, una escala validada para la población española en la que se evalúan los síntomas y su influencia en la calidad de vida mediante 31 preguntas ${ }^{20}$.

\subsubsection{Síntomas vasomotores}

Los síntomas vasomotores o sofocos se describen como una sensación de calor, percibida generalmente en la parte superior del tórax, el cuello y la cara, a menudo asociada con transpiración abundante. Su frecuencia, duración e intensidad es variable, aunque habitualmente duran menos de cinco minutos ${ }^{21}$. A menudo estos síntomas se presentan durante el sueño (sofocación nocturna) y propician el insomnio ${ }^{22}$. En ocasiones los ambientes calurosos, la ropa ajustada, las bebidas calientes y el estrés pueden desencadenarlos. Para muchas mujeres, estos episodios afectan a su calidad de vida y les llevan a consultar con su médico/a.

La clínica vasomotora aumenta en frecuencia e intensidad durante la transición menopáusica. Aunque estos síntomas pueden ir desapareciendo de forma paulatina en meses, en algunas mujeres pueden persistir durante varios años después del último periodo menstrual. La sintomatología suele ser de mayor intensidad en la menopausia quirúrgica ${ }^{23,24}$.

Los sofocos y la sudoración nocturna se asocian de manera consistente Calidad baja con la disminución de estrógenos, como han mostrado algunos estudios observacionales ${ }^{725-27}$. Los episodios de sudoración nocturna pueden provocar alteraciones del sueño $\mathrm{O}^{28}$, pero no se ha demostrado que el insomnio esté directamente asociado con la disminución de estrógenos ${ }^{7,29}$. 
Algunos problemas de salud deben considerarse en el diagnóstico diferencial de los síntomas vasomotore ${ }^{30}$ :

- El hipertiroidismo cursa con menstruaciones irregulares, sudores y cambios de humor.

- El hipotiroidismo da lugar a una sintomatología inespecífica que se puede solapar con la sintomatología menopáusica.

- Otras etiologías de cambios en el ciclo menstrual, como embarazo e hiperprolactinemia.

- Los sofocos y los episodios de sudoración profusa pueden ser debidos a la toma de ciertos medicamentos (diltiazem, levodopa) o a la existencia de otros trastornos como el síndrome carcinoide, feocromocitoma, infecciones (brucelosis, tuberculosis) o ciertos tipos de cáncer.

\subsubsection{Síntomas vaginales}

Los síntomas vaginales, como la sequedad, la irritación, el picor, el Calidad baja escozor y la dispareunia están relacionados con la atrofia vaginal asociada a la menopausia ${ }^{31}$.

Un estudio longitudinal con 438 mujeres observadas durante 7 años mostró que la sequedad vaginal aumentaba progresivamente a medida que la mujer se acercaba a la menopausia (de un $3 \%$ en la premenopausia a un $25 \%$ un año después de la menopausia y $47 \%$ tres años después de la menopausia $)^{7,29}$. Estos síntomas aparecen en alrededor del $30 \%$ de las mujeres en el periodo postmenopáusico inicial y hasta en un $47 \%$ de las mujeres en el periodo postmenopáusico tardío ${ }^{32}$.

\subsubsection{Síntomas urinarios}

Las infecciones urinarias de repetición no se ha demostrado que estén asociadas Calidad baja con la menopausia ${ }^{29}$. En mujeres durante la etapa de la postmenopausia los factores de riesgo principales para padecerlas son: prolapso vaginal, cistocele, residuo postmiccional, cambios en la flora vaginal e incontinencia urinaria ${ }^{33,34}$.

Aunque a menudo la incontinencia urinaria coincide en el tiempo con la menopausia, su etiología es compleja y multifactorial. Algunos estudios han mostrado una posible asociación con la menopausia ${ }^{26}$, pero otros atribuyen su desarrollo a factores mecánicos, como la obesidad, la cirugía ginecológica o la multiparidad ${ }^{7,35}$. La etiología de los factores hormonales en la función vesical y uretral no está plenamente establecida, y de hecho, no hay constancia de que haya mejoría con el tratamiento hormonal ${ }^{36}$. 


\subsubsection{Cambios del estado de ánimo}

Clásicamente se ha creído que la menopausia se asociaba a ansiedad, los Calidad baja cambios de humor, la irritabilidad y la depresión, sin embargo esta relación no se ha demostrado y existen estudios que sugieren que la mayoría de mujeres no sufren grandes cambios en el estado de ánimo durante la transición menopáusica ${ }^{37-40}$. Algunos estudios observacionales no han mostrado una asociación entre la transición menopáusica y la aparición de depresión mayor ${ }^{41-44}$, mientras que otros estudios han sugerido una relación entre ambos, así como entre la menopausia y el ánimo depresivo ${ }^{45-49}$.

\subsubsection{Trastornos cognitivos}

Los trastornos cognitivos, como la pérdida de memoria o la falta de Calidad baja concentración es poco probable que estén relacionados con la menopausia $^{38}$. A pesar de que se ha sugerido que el aporte de estrógenos podría proteger a la mujer del deterioro cognitivo ${ }^{50,51}$, se ha demostrado que la terapia hormonal no lo previene cuando se administra hasta un máximo de cinco años ${ }^{52}$ ni lo mejora en mujeres con demencia ${ }^{53}$.

No hay evidencia suficiente para determinar si algunos subgrupos de mujeres con tipos específicos de terapia hormonal se podrían beneficiar del tratamiento ${ }^{52}$, aunque algunos estudios sugieren que los estrógenos (sin gestágenos) podrían tener un efecto beneficioso en la memoria en mujeres $<65$ años $^{54}$.

\subsubsection{Disfunciones sexuales}

El deseo sexual hipoactivo puede relacionarse con la deficiencia hormonal, Calidad baja aunque otros factores como la depresión y la sequedad vaginal están también implicados en su desarrollo ${ }^{55,56}$. En cualquier caso, factores no hormonales, como conflictos con la pareja, insomnio, falta de estimulación, estrés o depresión son factores que contribuyen a su desarrollo y deberían tenerse en cuenta $^{39}$. En un estudio de cohortes, los problemas sexuales o de pareja tuvieron una influencia mayor que los niveles bajos de estrógenos en la función sexual ${ }^{57}$.

La disfunción sexual por dolor (dispareunia) guarda una directa relación con la atrofia vulvovaginal asociada a la carencia de estrógenos.

\subsubsection{Dolores musculares y articulares}

No se ha encontrado una asociación de los dolores musculares y Calidad baja articulares con la menopausia ${ }^{7}$. Aunque estos síntomas se han relacionado 
con una disminución de los estrógenos, la osteoartritis, la artritis reumatoide, la fibromialgia, los síndromes por somatización y los traumatismos mecánicos suelen ser sus causas habituales. En cualquier caso, no están relacionados con la pérdida de masa ósea ${ }^{58,59}$.

\subsubsection{Cambios en la piel}

En la transición climatérica, el contenido de colágeno de la piel y el grosor Calidad baja de la piel disminuyen. Por otra parte, la laxitud de la piel y las arrugas aumentan ostensiblemente. Es difícil separar los cambios relacionados con el envejecimiento, el tabaquismo y la exposición solar de los cambios relacionados con la disminución hormonal y la menopausia ${ }^{60}$.

\subsubsection{Aumento de peso}

El aumento de peso es poco probable que sea debido a la menopausia. El Calidad baja peso corporal en las mujeres tiende a aumentar con la edad, aunque algunos estudios muestran una mayor predisposición a la obesidad central en las mujeres durante la postmenopausia ${ }^{61-63}$. Un estudio longitudinal con 418 mujeres americanas mostró que el aumento de peso no se asoció con la transición menopáusica, sino con el estilo de vida ${ }^{64}$.

\section{Resumen de la evidencia - Manifestaciones clínicas}

\begin{tabular}{|c|c|}
\hline $\begin{array}{l}\text { Calidad } \\
\text { MUY } \\
\text { BAJA }\end{array}$ & $\begin{array}{l}\text { Existen varias escalas para evaluar los síntomas menopáusicos pero al } \\
\text { emplear distintos métodos no permiten comparaciones entre estudios y } \\
\text { a menudo no ajustan sus resultados por variables como la edad, } \\
\text { patología previa o el uso de terapia hormonal }{ }^{16-20} \text {. }\end{array}$ \\
\hline $\begin{array}{l}\text { Calidad } \\
\text { BAJA }\end{array}$ & $\begin{array}{l}\text { Los síntomas vasomotores }{ }^{7,25-27} \text { y los vaginales }{ }^{31} \text { son los síntomas más } \\
\text { claramente asociados con la menopausia. }\end{array}$ \\
\hline $\begin{array}{l}\text { Calidad } \\
\text { BAJA }\end{array}$ & $\begin{array}{l}\text { Las infecciones urinarias de repetición no están asociadas con la } \\
\text { menopausia. La incontinencia urinaria es compleja y multifactorial y su } \\
\text { posible asociación con la menopausia es controvertida }\end{array}$ \\
\hline $\begin{array}{l}\text { Calidad } \\
\text { BAJA }\end{array}$ & $\begin{array}{l}\text { Los cambios en el estado de ánimo }{ }^{37-40} \text {, los trastornos cognitivos }{ }^{38} \text {, las } \\
\text { disfunciones sexuales }{ }^{39,57} \text { (excepto la sequedad vaginal y la dispareunia } \\
\text { por ésta), los dolores musculares y articulares }{ }^{7} \text {, los cambios en la piel }{ }^{60} \text { y } \\
\text { el aumento de peso no están asociados con la menopausia }{ }^{61-64} \text {. }\end{array}$ \\
\hline
\end{tabular}




\section{Recomendación}

\begin{tabular}{|c|l|}
\hline \multirow{3}{*}{ BPC } & $\begin{array}{l}\text { Se sugiere informar a las mujeres de que la menopausia solo se ha } \\
\text { demostrado que se asocie a síntomas vasomotores y vaginales, y no a } \\
\text { otros síntomas que habitualmente se han creído asociados a la } \\
\text { menopausia como los síntomas urinarios, la ansiedad, los cambios de } \\
\text { humor, la irritabilidad y la depresión. }\end{array}$ \\
\hline
\end{tabular}




\title{
5. Tratamiento de los síntomas vasomotores
}

\author{
Preguntas para responder \\ En mujeres con síntomas vasomotores durante la peri y postmenopausia, \\ - ¿Cuál es la eficacia y la seguridad de la modificación en los estilos de vida? \\ - ¿Cuál es la eficacia y la seguridad de la terapia hormonal con estrógenos y \\ estrógenos combinados con gestágenos? \\ - ¿Cuál es la eficacia y la seguridad de la terapia hormonal con gestágenos? \\ - ¿Cuál es la eficacia y la seguridad de la terapia hormonal con andrógenos? \\ - ¿Cuál es la eficacia y la seguridad de la terapia hormonal con tibolona? \\ - ¿Cuál es la eficacia y la seguridad de los antidepresivos? \\ - ¿Cuál es la eficacia y la seguridad de otros fármacos como la gabapentina, metildopa \\ y clonidina? \\ - ¿Cuál es la eficacia y la seguridad de la fitoterapia? \\ - ¿Cuál es la eficacia y la seguridad de los tratamientos no farmacológicos? \\ - ¿Cuál es la eficacia y la seguridad de otras terapias alternativas?
}

En este apartado se revisan las distintas intervenciones propuestas para mejorar los síntomas vasomotores.

\subsection{Intervenciones en los estilos de vida}

El estudio observacional 'Study of Women's Health Across the Nation' ${ }^{65,66}$ ha mostrado una asociación entre determinados factores de riesgo y estilos de vida con los síntomas vasomotores. Algunos factores de riesgo identificados (raza, edad, factores socioeconómicos, etc.) no son modificables, mientras que otros (estilos de vida o IMC) sí son susceptibles de ser sometidos a unas estrategias de prevención.

\subsection{1. Índice de masa corporal (IMC)}

En una revisión narrativa ${ }^{67}$ se identificaron seis estudios que analizaban la Calidad baja relación entre el IMC y los síntomas vasomotores. Cinco de éstos hallaron que un mayor IMC estaba asociado con una mayor notificación de síntomas vasomotores. Cuando el IMC era analizado como una variable continua, la odds ratio (OR) ajustada de síntomas vasomotores aumentaba de un 
$5 \%$ a un $10 \%$ por unidad de IMC. Cuando el IMC era analizado por categorías, las mujeres con un IMC $>25$ presentaban un riesgo 1,7 veces mayor de presentar síntomas vasomotores que aquellas con un IMC $\leq 22$.

El aumento de la adiposidad se ha relacionado con una disminución de los síntomas vasomotores durante la menopausia, debido a la conversión de andrógenos en estrógenos en el tejido adiposo. Sin embargo, los últimos modelos de termorregulación, postulan que el aumento de tejido adiposo se asocia con una mayor probabilidad de síntomas vasomotores ${ }^{68-70}$. Los resultados del estudio observacional Study of Women's Health Across the Nation ${ }^{68}$ (muestra de 1.776 mujeres) también mostraron que un mayor porcentaje de grasa corporal se asoció con una mayor probabilidad de presentar síntomas vasomotores (por aumento de la desviación estándar en el porcentaje de grasa corporal, OR 1,27, intervalo de confianza (IC) $95 \% 1,14$ a 1,42) en modelos ajustados por edad y lugar.

En un ensayo clínico aleatorizado (ECA) en el que se evaluó, de forma secundaria, la eficacia de la pérdida de peso (mediante ejercicio y dieta hipocalórica) sobre los sofocos, se concluyó que la reducción de peso, IMC y circunferencia abdominal se asociaba a una disminución modesta de los sofocos; sin embargo, esta asociación no era significativa cuando el análisis se ajustaba por posibles factores de confusión (OR 1,92; IC $95 \% 0,95-3,89)^{71}$.

\subsubsection{Tabaco}

En una revisión narrativa ${ }^{67}$ se identificaron diversos estudios observacionales Calidad baja que mostraban un aumento del riesgo de sofocos en mujeres fumadoras. La mayoría de estudios mostraban una mayor OR (rango de 1,5 a 2,0) en la asociación de fumar con síntomas vasomotores. Aunque el único estudio que controlaba por la exposición al humo de tabaco encontró que el tabaquismo activo no era un factor de predicción de los síntomas vasomotores.

\subsubsection{Actividad física}

La revisión sistemática Cochrane de Daley et al., de $2007^{72}$ identificó un sólo Calidad baja $\mathrm{ECA}^{73}$ que incluía 25 mujeres (sedentarias, con síntomas vasomotores y de 48 a 63 años) y que comparaba una intervención de ejercicio físico (12 semanas de sesiones de ejercicio de intensidad moderada) frente al tratamiento hormonal. El estudio mostró en el análisis intragrupo que las dos intervenciones fueron efectivas en la reducción de los síntomas vasomotores, aunque el análisis entre grupos observó que el grupo con terapia hormonal experimentó significativamente menos sofocos en comparación con el grupo de ejercicio durante el seguimiento (diferencia media (DM) del número de sofocos cada 24 horas a las 36 semanas: 3,0, IC $95 \% 0,77$ a 5,22). 
Se ha identificado un ECA posterior con limitaciones Calidad metodológicas ${ }^{74}$ que evalúa el efecto de los estrógenos y el ejercicio físico muy baja de moderada intensidad (bicicleta estática 3 veces/semana) sobre la calidad de vida. El estudio incluyó 44 mujeres sin obesidad que habían sido sometidas a histerectomía y que no recibían terapia hormonal. Las participantes fueron aleatorizadas en 4 grupos durante 6 meses: ejercicio y terapia hormonal, no ejercicio y terapia hormonal, ejercicio y placebo, no ejercicio y placebo. Se observó una disminución de los sofocos después de la intervención en todos los grupos, pero sin diferencias entre ellos.

En un ECA también posterior $(\mathrm{N}=176)$ se ha evaluado la eficacia Calidad del ejercicio aeróbico (50 minutos, 4 veces/semana) frente a un grupo moderada / control sin ejercicio, en términos de frecuencia de los síntomas muy baja vasomotores, la calidad de vida ${ }^{75}$, prevalencia de la sudoración nocturna ${ }^{76}$ y calidad del sueño en relación con los sofocos nocturnos ${ }^{77}$. Los resultados mostraron una mayor disminución de los síntomas vasomotores nocturnos $^{75}$, siendo el ejercicio significativamente más eficaz en la reducción de los sudores nocturnos ${ }^{76,77}$.

La revisión sistemática de Lee et al., de $2009^{78}$ identificó dos ECA Calidad baja (232 mujeres) que comparaban los efectos del yoga frente a andar o realizar ejercicio físico. El yoga no se mostró significativamente superior a la actividad física (diferencia de medias 0,34, IC $95 \%-0,27$ a 0,96).

\subsubsection{Otros factores}

Algunos estudios observacionales muestran que los sofocos pueden Calidad baja desencadenarse por un aumento de la temperatura ambiente, el uso de ropas ajustadas, el consumo de bebidas calientes o alcohol o el estrés ${ }^{79}$. Sin embargo, no se han identificado ECA que muestren si evitar estas situaciones o el consumo de estas bebidas disminuyen el riesgo de aparición de sofocos. 
Resumen de la evidencia - intervenciones en los estilos de vida

\begin{tabular}{|c|c|}
\hline \multicolumn{2}{|r|}{ IMC } \\
\hline $\begin{array}{c}\text { Calidad } \\
\text { MUY BAJA }\end{array}$ & $\begin{array}{l}\text { El sobrepeso/obesidad se asocia a un mayor riesgo de presentar } \\
\text { síntomas vasomotores }^{67-70} \text {. }\end{array}$ \\
\hline \multicolumn{2}{|r|}{ Tabaco } \\
\hline $\begin{array}{l}\text { Calidad } \\
\text { BAJA }\end{array}$ & $\begin{array}{l}\text { El tabaquismo se asocia a un mayor riesgo de presentar síntomas } \\
\text { vasomotores }{ }^{67} \text {. }\end{array}$ \\
\hline \multicolumn{2}{|r|}{ Actividad física } \\
\hline $\begin{array}{l}\text { Calidad } \\
\text { BAJA }\end{array}$ & $\begin{array}{l}\text { El ejercicio físico se ha mostrado moderadamente efectivo para la } \\
\text { reducción de los síntomas vasomotores, es más eficaz que placebo y } \\
\text { menos eficaz que la terapia hormonal }{ }^{72-74} \text {. }\end{array}$ \\
\hline $\begin{array}{l}\text { Calidad } \\
\text { MODERADA } \\
\text { / MUY BAJA }\end{array}$ & $\begin{array}{l}\text { El ejercicio físico disminuye ligeramente los síntomas vasomotores } \\
\text { nocturnos, especialmente la sudoración }{ }^{75-77} \text {. }\end{array}$ \\
\hline $\begin{array}{l}\text { Calidad } \\
\text { BAJA }\end{array}$ & $\begin{array}{l}\text { La práctica de yoga no se ha mostrado significativamente superior a } \\
\text { la actividad física para la reducción de los síntomas vasomotores }{ }^{78} \text {. }\end{array}$ \\
\hline \multicolumn{2}{|r|}{ Otros factores } \\
\hline $\begin{array}{l}\text { Calidad } \\
\text { BAJA }\end{array}$ & $\begin{array}{l}\text { Evitar bebidas calientes, cafeína, bebidas alcohólicas o ambientes } \\
\text { calurosos en general podría disminuir la frecuencia y/o la intensidad } \\
\text { de los sofocos }{ }^{79} \text {. }\end{array}$ \\
\hline
\end{tabular}

\section{Recomendaciones}

\begin{tabular}{|c|l|}
\hline FUERTE & $\begin{array}{l}\text { En mujeres que presentan síntomas vasomotores durante el } \\
\text { climaterio, en la etapa de la perimenopausia y postmenopausia, se } \\
\text { recomienda evitar la obesidad, realizar ejercicio físico y evitar el } \\
\text { consumo de tabaco para reducir los síntomas vasomotores. }\end{array}$ \\
\hline DÉBIL & $\begin{array}{l}\text { En mujeres con síntomas vasomotores durante la peri y } \\
\text { postmenopausia se sugiere procurar un ambiente fresco, disminuir } \\
\text { el consumo de cafeína, bebidas calientes, alcohol y comidas } \\
\text { picantes para no exacerbar la sintomatología. }\end{array}$ \\
\hline
\end{tabular}




\subsection{Intervenciones farmacológicas}

\subsubsection{Tratamiento hormonal}

\subsubsection{Estrógenos solos y combinados}

Se ha incluido una RS que evaluó el efecto de la terapia hormonal oral (administrada entre tres meses y tres años) ${ }^{80}$ en comparación con placebo (24 ECA, 3.329 mujeres), evaluando la frecuencia y la intensidad de los sofocos. El rango de edad de las participantes en los estudios incluidos en esta revisión osciló entre los 34 y 64 años con una edad media en la mayoría de los ensayos de aproximadamente 50 años. No se proporcionó información en cuanto a la edad de inicio de los tratamientos. Además, se identificaron tres nuevas RS que evaluaron el balance riesgo/beneficio de la terapia hormonal con estrógenos solos y combinados: dos evaluaron sus efectos (beneficiosos y desfavorables) a largo plazo en mujeres en la etapa de la peri y postmenopausia ${ }^{81,82}$ y una el riesgo de cáncer de pulmón asociado a su uso ${ }^{83}$.

También se han incluido estudios que evalúan el tipo de estrógeno administrado ${ }^{84-87}$, diferentes tipos y dosis de combinaciones de estrógenos más gestágenos ${ }^{88-93}$ y la combinación de estrógenos con bazedoxifeno ${ }^{94}$. Debido a la variabilidad entre los estudios, es difícil valorar comparativamente el efecto de los diferentes tipos, dosis y combinaciones del tratamiento hormonal.

Tres de estos estudios identificados fueron tres ECAs recientes ${ }^{84,88,94}$ y un análisis secundario del estudio $\mathrm{WHI}^{85}$, que evaluaron la eficacia de diferentes tipos y dosis de estrógenos solos y combinados: estrógenos $\operatorname{solos}^{84,85}$, estrógenos con gestágenos ${ }^{88}$ y estrógenos con bazedoxifeno ${ }^{94}$. Debido a la variabilidad entre ellos, es dificil realizar análisis comparativos. Además, se ha identificado un nuevo ECA sobre estradiol trasdérmico ${ }^{95}$.

Uno de estos ECA incluidos, a doble-ciego de 12 semanas $^{88}$ evaluó la eficacia de diferentes combinaciones de estrógenos esterificados (dosis bajas) con metiltestosterona para reducir los sofocos (frecuencia e intensidad), frente a placebo, en 1.288 mujeres en la etapa de la postmenopausia. No se presentaron resultados numéricos sino sólo los valores de significación estadística y no se presentaron resultados finales (semana 12), sino en tiempos parciales (semanas 4 y 5 ).

Otro ECA doble-ciego de 12 semanas $^{84}$ evaluó la eficacia de dos dosis de estrógenos-B sintéticos conjugados $(0,3$ y $0,625 \mathrm{mg} / \mathrm{d})$ para reducir la frecuencia de sofocos y sudores nocturnos, frente a placebo, en 157 mujeres en la etapa de la postmenopausia (30-65 años). Adicionalmente se evaluó la calidad del sueño valorada subjetivamente por las pacientes. La variable primaria fue la reducción media (frecuencia semanal) de sudores nocturnos que causan despertar en las pacientes desde el inicio al final del tratamiento.

Un ECA doble-ciego ${ }^{94}$ evaluó la eficacia de la combinación de estrógenos conjugados $(0,45$ y $0,625 \mathrm{mg} / \mathrm{d})$ con bazedoxifeno $(20 \mathrm{mg} / \mathrm{d})$, para reducir los sofocos, frente a placebo, en 318 mujeres en la etapa de la postmenopausia no histerectomizadas (40-65 años). 
Las principales variables fueron: el número medio de días/semana sin sofocos moderadosgraves y libres de sofocos de cualquier tipo, desde el inicio hasta el final del tratamiento a las 12 semanas. Adicionalmente, se presentó la proporción de mujeres que no experimentaron ningún cambio en el número de sofocos moderados-graves durante el tratamiento. Este estudio fue un análisis secundario de los datos del estudio SMART-2 (Selective Estrogens, Menopause, and Response to Therapy) publicado con anterioridad, sobre el efecto de esta combinación en la frecuencia y gravedad de los sofocos.

Un análisis secundario del estudio WHI 1998 (Women's Health Initiative), evaluó la eficacia de los estrógenos conjugados equinos (ECC 0,625 mg/d) para reducir los sofocos y sudores nocturnos, frente a placebo, en 10.739 mujeres en la etapa de la postmenopausia tras un año de tratamiento ${ }^{85}$. También evaluó el efecto del tratamiento sobre otros síntomas asociados a la menopausia y, de forma complementaria, realizó un seguimiento (estudio de cohorte) de las mujeres que continuaron con el tratamiento hormonal tras la finalización del estudio WHI, analizando la evolución de la sintomatología, los efectos de la suspensión del tratamiento y posibles factores influyentes. Los resultados se expresan como la proporción de mujeres que presentan cada uno de los síntomas o efectos analizados tras un año de tratamiento, calculados en función de la presencia o ausencia de sintomatología basal; lo que dificultó la realización de comparaciones con otros estudios.

\subsection{Terapia hormonal frente a placebo}

Se ha encontrado una reducción significativa en la frecuencia semanal Calidad de sofocos con la terapia hormonal (diferencia media ponderada moderada (DMP) -17,92; IC $95 \%-22,86$ a -12,99) frente a placebo. La intensidad de la sintomatología también se redujo significativamente en comparación con placebo (OR 0,13; IC $95 \%$ 0,06 a 0,27). La magnitud del efecto se relacionó con la intensidad de los síntomas y la duración del tratamiento. Se observó una mayor reducción de la sintomatología con los estrógenos combinados con gestágenos en comparación con placebo (OR 0,10; IC $95 \% 0,06$ a 0,19$)$, y con los estrógenos comparados con placebo $(\text { OR } 0,35 \text {; IC } 95 \% 0,22 \text { a } 0,56)^{80}$.

\subsection{Dosis baja de estrógenos frente a placebo}

La dosis efectiva más baja de estrógenos en el tratamiento de los síntomas vasomotores es todavía motivo de debate/estudio. Considerando $<1 \mathrm{mg} /$ día de estradiol (o 0,3 mg de conjugados equinos) como dosis baja, se han identificado cuatro estudios que comparan dosis bajas de estrógenos para el tratamiento de los síntomas vasomotores ${ }^{96-99}$. Después de la publicación del estudio $\mathrm{WHI}^{100}$, no se ha identificado ninguna $\mathrm{RS}$ ni nuevos estudios que evalúen la eficacia de dosis bajas de estrógenos en el tratamiento de mujeres en la etapa postmenopaúsica.

Las dosis bajas o ultrabajas de estradiol $(25 \circ \mathrm{gg} / \mathrm{d}$ o $14 \circ \mathrm{cg} / \mathrm{d}$ de Calidad estrógenos respectivamente), en parche o gel, comparadas con placebo moderada mejoran los síntomas vasomotores en mujeres durante la etapa de la postmenopausia $^{96-99}$. 


\subsection{Vía de administración}

El tratamiento hormonal se encuentra disponible por vía oral, transdérmica (gel, parches, implantes), intranasal y vaginal. En España no está comercializada la vía intranasal ni la vaginal para terapia sistémica. Se identificaron $11 \mathrm{ECA}$, dos $\mathrm{ECA}^{101,102}$ analizando la eficacia entre el estradiol intranasal (300 ๙ g/día) y transdérmico (50 ๙ g/día), cinco ${ }^{103-107}$ que compararon la vía oral y la nasal y cuatro que compararon la vía oral y transdérmica ${ }^{108-111}$.

Adicionalmente se identificaron dos ECAs doble-ciego, publicados de forma conjunta $^{95}$, que evaluaron la eficacia de diferentes dosis de estradiol transdérmico (gel) en mujeres en la etapa de la postmenopausia, para establecer la dosis más eficaz y segura en el tratamiento de los sofocos (frecuencia e intensidad) y la sintomatología vaginal. El primer estudio en fase $3(n=221)$, evaluó el gel de estradiol $(0,06 \%)$ en dosis de $1,5 \mathrm{mg}(2,5 \mathrm{~g}$ de gel) y $0,75(1,25 \mathrm{~g}$ de gel); y el segundo en fase $4(\mathrm{n}=351)$, el gel de estradiol $(0,03 \%)$ en dosis de $0,375 \mathrm{mg}(1,25 \mathrm{~g}$ de gel $)$ y $0,27 \mathrm{mg}(0,9 \mathrm{~g}$ de gel $)$. La variable principal de medida en ambos estudios fue la diferencia en la frecuencia de los sofocos (moderados-graves) desde el inicio al final del tratamiento a las 12 semanas, y la secundaria la frecuencia y la gravedad de los sofocos totales. En el estudio en fase 4 se consideró también como variable primaria la diferencia media en la frecuencia y la gravedad de los síntomas vasomotores (moderadosgraves) desde el inicio del tratamiento, hasta las semanas 4 y 12.

Tras 12 semanas de tratamiento, el estradiol transdérmico (gel Calidad $0,06 \%$ y $0,03 \%$ ) fue significativamente más eficaz que placebo para muy baja aliviar la frecuencia de los sofocos en mujeres durante la etapa de la postmenopausia, siendo el efecto dosis-dependiente ( 2 ECA en fase 3 y 4 , 221 mujeres en fase 3 y 351 en fase 4,12 semanas $^{95}$ ).

* Gel 0,06 \%:

- 1,5 mg estradiol: DME -2,72; $\mathrm{p}<0,001$.

- 0,75 mg estradiol: DME -1,71; $\mathrm{p}<0,05$.

* Gel 0,03 \%:

- 0,375 mg estradiol: DME -3,82; $\mathrm{p}<0,001$.

- 0,27 mg estradiol: DME -1,45; $\mathrm{p}<0,05$.

Se ha observado una eficacia comparable entre $17 \beta$-estradiol Calidad baja intranasal (300 og/día) y 17ß-estradiol transdérmico $(50 \propto \mathrm{g} / \mathrm{día})^{101,102}$. La vía oral y la nasal y la vía oral y transdérmica han presentado una eficacia semejante.

\subsection{Pauta de administración}

Cuatro ECAs (538 mujeres) han evaluado las pautas de administración del tratamiento hormonal, diferenciando un régimen combinado continuo (estrógenos más gestágenos) o un régimen combinado secuencial (primero estrógenos y posteriormente estrógenos más gestágenos) $)^{112-115}$. 
Ambos regímenes mejoraron los síntomas vasomotores, aunque se observaron Calidad baja diferencias en otras medidas de resultado (riesgo de sangrado, problemas del sueño o función cognitiva) ${ }^{112-115}$.

\subsection{Retirada del tratamiento}

Se han identificado cuatro estudios que evalúan diferentes pautas de retirada del tratamiento hormonal en relación a la recurrencia de los síntomas vasomotores. Uno de los estudios analizaba una disminución gradual de la dosis ${ }^{116}$, en cambio, los otros analizaban una disminución en el número de días de tratamiento ${ }^{117-119}$.

Las diferentes pautas de retirada del tratamiento hormonal no Calidad baja disminuyeron la recurrencia de los síntomas al final del tratamiento sino que parecen posponer su aparición.

\subsection{Balance riesgo/beneficio}

Se identificaron $12 \mathrm{RS}^{80,120-130}$ que analizaron los efectos adversos y la seguridad de la terapia hormonal sustitutiva (THS) (con estrógenos combinados o no con progestágenos) en mujeres durante la etapa postmenopáusica y perimenopáusica. No obstante, la mayoría de los resultados $(70 \%)$ proceden de dos grandes ECA: estudio HERS 1998 (Estrogen/progestin Replacement Study) y estudio WHI 1998 (Women's Health Initiative). Adicionalmente, se han identificado 3 RS más recientes ${ }^{81-83}$ que se describen a continuación.

Una RS con metanálisis (23 ECA doble-ciego, 42.830 mujeres de 26-91 años, con menopausia espontánea o quirúrgica, con al menos un grado de comorbilidad de las variables consideradas) evaluó el efecto a largo plazo de la terapia hormonal sobre la mortalidad, las variables cardiovasculares, el cáncer, los trastornos de la vesícula biliar, los trastornos cognitivos, las fracturas y la calidad de vida; durante y después de la terapia $^{82}$. El efecto de la terapia hormonal también se analizó en función de la edad y determinadas situaciones de morbilidad previa o concomitante. Las terapias hormonales administradas fueron estrógenos solos o combinados con gestágenos por vía oral (régimen contínuo o secuencial), transdérmica o intranasal durante al menos un año. Se excluyeron los ECAs en los que se evaluaron cremas, comprimidos vaginales tópicos y anillos. La TH con estrógenos solos incluyó: 17-B estradiol, estradiol valerato, estradiol transdérmico (parches), 17-B estradiol intranasal $\mathrm{O}$ estrógenos equinos conjugados. La TH combinada incluyó alguno de los estrógenos anteriores + gestágenos: medroxiprogesterona acetato, didrogesterona, noretisterona, progesterona micronizada. En la mayoría de los ECAs se utilizaban dosis moderadas de estrógenos (estradiol $1 \mathrm{mg} / \mathrm{d}$, estrógenos equinos conjugados o EEC 0,625 mg/d o estradiol transdérmico $0,05 \mathrm{mg} 2$ veces/semana). Las variables consideradas fueron: mortalidad total y por causa específica, eventos coronarios (infarto de miocardio o muerte por causa $\mathrm{CV}$ ), ictus o ataque isquémico transitorio, tromboembolismo venoso (TVP o EP), cáncer de mama, colorrectal, de pulmón, endometrio u ovario, trastornos de la vesícula biliar, fracturas, alteraciones de la función cognitiva y/o demencia y calidad de vida. No se consideraron variables relacionadas con la eficacia de la $\mathrm{TH}$ para reducir los síntomas vasomotores. 
Otra RS ${ }^{81}$ analizó 9 ECA doble-ciego que incluían un total de 27.347 mujeres durante la postmenopausia susceptibles de tratamiento con TH. Su objetivo fue evaluar la eficacia de la TH a largo plazo para reducir el riesgo de determinadas situaciones crónicas y sus efectos adversos. Además, se realizó un análisis en diferentes subgrupos poblacionales: menopausia prematura o quirúrgica; edad; tipo, pauta y forma de administración de la TH y comorbilidad. La mayoría de las variables estudiadas coincidieron con las de la revisión anterior ${ }^{82}$ y también se presentan los datos relativos al efecto de la TH sobre la incontinencia urinaria (no incluidos en la RS anterior); y de forma complementaria, se calculó un índice global de riesgos y beneficios, que agrupa los resultados relativos a eventos coronarios, cáncer de mama, ictus, embolia pulmonar, cáncer colorrectal, fractura de cadera y muerte por cualquier causa.

Una RS con metanálisis ${ }^{83}$ analizó 18 estudios (9 caso-control, 4 cohortes, 3 ECA, 2 de registros de casos) para evaluar el impacto de la TH en el riesgo de cáncer de pulmón y sus diferentes tipos histológicos. Solo se incluyeron estudios disponibles a texto completo. Se incluyeron estudios con TH con estrógenos solos y/o asociados a gestágenos, sin especificar cuál; en algunos estudios, se desconoce el tipo de TH administrada. Los subtipos histológicos del cáncer de pulmón son también diferentes según los estudios y la población de pacientes es variada, incluyendo mujeres fumadoras y no fumadoras. No se especifica el número de pacientes incluidos, su edad, ni la duración de los estudios y solo dos de los tres ECA analizados son comparativos (frente a placebo).

La RS de Maclennan no mostró un aumento significativo del Calidad abandono del tratamiento a causa de los efectos adversos (dolor mamario, moderada edema, dolores articulares y síntomas psicológicos), aunque la presencia de efectos adversos fue superior con la terapia hormonal en comparación con placebo (OR 1,41; IC $95 \% 1,00$ a 1,99). Otros efectos adversos frecuentes fueron el sangrado vaginal con la terapia hormonal combinada continua y las náuseas ${ }^{80}$.

En mujeres en la etapa de la postmenopausia relativamente sanas, la Calidad terapia combinada continua con estrógenos equinos conjugados moderada $(0,625 \mathrm{mg} / \mathrm{d})$ y acetato de medroxiprogesterona $(2,5 \mathrm{mg} / \mathrm{d})$, aumentó significativamente el riesgo de eventos coronarios ( 1 año de tratamiento, RA $4 \%$, IC $95 \% 3$ a 7), tromboembolismo venoso (1 año de tratamiento, RA $7 \%$, IC $95 \% 4$ a 11), ictus (3 años de tratamiento, RA $18 \%$, IC $95 \% 14$ a 23), cáncer de mama (5,6 años de tratamiento, RA $23 \%$, IC $95 \% 19$ a 29), trastornos de la vesícula biliar (5,6 años de tratamiento, RA $27 \%$, IC $95 \% 21$ a 34) y muerte por cáncer de pulmón (5,6 años de tratamiento mas 2,4 años de seguimiento, RA $9 \%$, IC $95 \% 6$ a 13$)^{82}$.

En mujeres en la etapa de la postmenopausia mayores de 65 años Calidad relativamente sanas, la terapia combinada continua con estrógenos moderada equinos conjugados $(0,625 \mathrm{mg} / \mathrm{d})$ y acetato de medroxiprogesterona $(2,5 \mathrm{mg} / \mathrm{d})$, aumentó significativamente la incidencia de demencia (4 años de tratamiento, Riesgo Absoluto (RA) $18 \%$, IC $95 \% 11$ a 30) ${ }^{82}$. 
En mujeres en la etapa de la postmenopausia con enfermedad Calidad cardiovascular, la terapia combinada continua con estrógenos (estrógenos moderada equinos conjugados $0,625 \mathrm{mg} / \mathrm{d}$ ) y acetato de medroxiprogesterona $(2,5 \mathrm{mg} / \mathrm{d})$, aumentó significativamente el riesgo de tromboembolismo venoso ( 1 año de tratamiento, RA $9 \%$, IC $95 \% 3$ a 29) ${ }^{82}$.

La TH combinada continua con estrógenos equinos conjugados Calidad $(0,625 \mathrm{mg} / \mathrm{d})$ y acetato de medroxiprogesterona $(2,5 \mathrm{mg} / \mathrm{d})$ a largo plazo en moderada mujeres en la etapa de la postmenopausia sanas (50-79 años), aumentó significativamente la incontinencia urinaria (de estrés, urgencia o mixta) tras 1 año de tratamiento: HR 1,39, IC $95 \%$ 1,27 a 1,52 (1 ECA; 16.608 mujeres; 5,2 años; 872 eventos más por 10.000 mujeres-año; IC $95 \% 591$ a 1153) ${ }^{81}$.

En mujeres en la etapa de la postmenopausia relativamente sanas, la Calidad terapia con estrógenos sin gestágenos (estrógenos equinos conjugados moderada $0,625 \mathrm{mg} / \mathrm{d}$ ), aumentó significativamente el riesgo de tromboembolismo venoso (1-2 años de tratamiento, RA $5 \%$, IC $95 \% 2$ a 10; 7 años de tratamiento, RA $21 \%$, IC $95 \% 16$ a 28), ictus (7 años de tratamiento, RA $32 \%$, IC $95 \% 25$ a 40) y trastornos de la vesícula biliar (7 años de tratamiento, RA $45 \%$, IC $95 \% 36$ a 57). Sin embargo, no se encontraron diferencias significativas en cuanto al riesgo de sufrir cáncer de mama ${ }^{82}$.

La TH con estrógenos sin gestágenos (estrógenos equinos conjugados Calidad $0,625 \mathrm{mg} / \mathrm{d}$ ) a largo plazo en mujeres sanas histerectomizadas (50-79 años), moderada aumentó significativamente la incontinencia urinaria (de esfuerzo, urgencia o mixta) tras 1 año de tratamiento: Hazard ratio (HR) 1,53, IC $95 \%$ 1,37 a 1,71 (1 ECA, 10.739 mujeres, 6,8 años; 1.271 eventos más por 10.000 mujeres-año, IC $95 \% 883$ a 1660). Sin embargo, no se encontraron diferencias significativas en cuanto al riesgo de sufrir cáncer de mama ${ }^{81}$.

La TH combinada continua con estrógenos equinos conjugados Calidad $(0,625 \mathrm{mg} / \mathrm{d})$ y acetato de medroxiprogesterona $(2,5 \mathrm{mg} / \mathrm{d})$ a largo plazo moderada en mujeres en la etapa de la postmenopausia con enfermedad arterial coronaria no histerectomizadas ( $\leq 80$ años), aumentó significativamente la incontinencia urinaria (de estrés o urgencia): (1 ECA, 2.763 mujeres, 4,1 años, OR 1,6; IC $95 \% 1,3$ a 1,71) ${ }^{81}$.

La TH con estradiol trasdérmico $(0,014 \mathrm{mg} / \mathrm{d})$ a largo plazo en Calidad mujeres en la etapa de la postmenopausia no histerectomizadas y con moderada densidad mineral ósea normal, no modificó significativamente el riesgo de sufrir incontinencia urinaria (1 ECA, 417 mujeres, 2 años $)^{81}$.

La TH con estrógenos asociados a gestágenos aumentó Calidad muy significativamente el riesgo de cáncer de pulmón en mujeres en la etapa baja de la menopausia (2 ECAs; RR 1,359; IC $95 \%$ :1,031 a 1,791) ${ }^{83}$.

No hay evidencias suficientes de que la TH tenga un impacto Calidad clinicamente significativo en la incidencia de cáncer colorectal ${ }^{82}$ moderada 
Además de las revisiones de Marjoribank ${ }^{82}$ y Nelson $^{81}$, que abordan un amplio abanico de resultados relevantes para la salud de las mujeres en la etapa de la postmenopausia y cuyos resultados principales se han presentado en los párrafos anteriores, se han encontrado otras revisiones con objetivos más restringidos, que abordan específicamente el efecto de las terapias hormonales en áreas concretas de la salud de mujeres.

La RS de Shah $^{123}$ analizó el efecto en relación al cáncer de mama e incluyó solamente estudios observacionales, la RS de Sare ${ }^{126}$ analizó el efecto en relación a las enfermedades cardiovasculares (31 ECA con 44.113 mujeres). Por otra parte, la RS de Canonico ${ }^{127}$ analizó el efecto en relación a los tromboembolismos venosos (497 eventos; 9 ECA y 15 estudios observacionales) y la elaborada por Allen ${ }^{130}$ analizó el efecto en el área de la salud dental (20 estudios -de cualquier diseño- con 13.735 mujeres). Además, la RS de Greiser ${ }^{124}$ analizó el impacto de la terapia hormonal (estrógenos solos o combinados con gestágenos) sobre el riesgo de cáncer de pulmón y sus diferentes tipos histológicos en mujeres en la etapa de la perimenopausia (9 estudios caso-control, 4 cohortes, 3 ECA, 2 de registros de casos).

En mujeres que usaron estrógenos solos ${ }^{123}$ (ocho estudios de cohortes Calidad y cinco estudios de casos y control, 700.000 mujeres) estimaron un OR de moderada sufrir cáncer de mama de 1,16 (IC $95 \%$ 1,06 a 1,28) para usuarias de terapia hormonal; para menos de 5 años de uso ( 9 estudios) el OR era 1,16 (IC $95 \% 1,02$ a 1,32) y para más de cinco años de uso (10 estudios), 1,20 (IC $95 \%$ 1,06 a 1,37). Para usuarias de terapia combinada (4 estudios de cohortes y cuatro de casos y control, 650.000 mujeres) estimaron un OR de 1,39 (IC $95 \%$ 1,12 a 1,72); para menos de 5 años de uso (seis estudios) el OR era 1,35 (IC $95 \% 1,16$ a 1,57) y para más de cinco años (seis estudios), 1,63 (IC $95 \%$ 1,22 a 2,18).

La terapia hormonal se asocia a un riesgo aumentado de accidente Calidad cerebrovascular (1.034 eventos; OR 1,32 ; IC $95 \% 1,14$ a 1,53) y de moderada tromboembolismo venoso (OR 2,05; IC $95 \%$ 1,44 a 2,92), así como de gravedad de accidente cerebrovascular (OR 1,31; IC $95 \%$ 1,12 a 1,54). El uso de terapia combinada con progesterona duplicaba el riesgo de tromboembolismo venoso ${ }^{126}$.

El riesgo de sufrir tromboembolismos venosos fue superior para Calidad usuarias de terapia estrogénica oral (RR 2,1; IC $95 \%$ 1,4 a 3,1) y no para moderada la terapia transdérmica $(1 \text { metanálisis })^{127}$.

No se encontraron diferencias significativas respecto a la salud Calidad dental, medida por variables como el número de dientes naturales moderada conservados y necesidad de dentadura postiza ${ }^{130}$.

El metanálisis de los resultados mostró que la terapia con estrógenos Calidad asociados a gestágenos aumentó significativamente el riesgo de cáncer de muy baja pulmón (RR 1,359; IC 95 \% 1,031 a 1,791) $)^{124}$. 


\subsection{Hormonas bioidénticas}

En EE.UU. existen las llamadas 'hormonas bioidénticas' que consisten en fórmulas magistrales con hormonas químicamente idénticas a las producidas por la mujer como el 17beta-estradiol o la progesterona, de venta en farmacias. Para ver los niveles en sangre se utilizan tests de saliva. Estos compuestos pueden presentar distintas dosis e ingredientes y no se ha demostrado su eficacia ni se conoce su seguridad ${ }^{131}$.

\section{Resumen de la evidencia - estrógenos y estrógenos combinados con gestágenos}

\begin{tabular}{|c|l|}
\hline Calidad & $\begin{array}{l}\text { El tratamiento hormonal con estrógenos o estrógenos combinados } \\
\text { con gestágenos es efectivo para disminuir la frecuencia y la } \\
\text { intensidad de los sofocos }{ }^{80} \text { frente a placebo. }\end{array}$ \\
\hline Calidad & $\begin{array}{l}\text { En cuanto a la seguridad, la terapia hormonal combinada continua } \\
\text { aumenta significativamente el riesgo de tromboembolismo venoso, } \\
\text { eventos cardiacos (infarto de miocardio o muerte cardiaca) e } \\
\text { incontinencia urinaria después de un año de uso }\end{array}$ \\
Mratamiento, el riesgo de ictus aumenta a los tres años y el de cáncer de \\
mama, trastornos de la vesícula biliar y mortalidad por cáncer de \\
pulmón a los 3-5,6 años. En mujeres mayores de 65 años, aumenta la \\
incidencia de demencia tras 4 años de uso
\end{tabular}




\begin{tabular}{|l|l|}
\hline $\begin{array}{l}\text { Calidad } \\
\text { BAJA }\end{array}$ & $\begin{array}{l}\text { La administración del tratamiento hormonal con un régimen } \\
\text { combinado continuo (estrógenos más gestágenos) o un régimen } \\
\text { combinado secuencial (primero estrógenos y posteriormente } \\
\text { estrógenos más gestágenos) ha mostrado una eficacia similar }{ }^{112-115}\end{array}$ \\
\hline $\begin{array}{l}\text { Calidad } \\
\text { BAJA }\end{array}$ & $\begin{array}{l}\text { Las diferentes pautas de retirada del tratamiento (disminución } \\
\text { gradual de la dosis o bien disminución en el número de días de } \\
\text { tratamiento) posponen la recurrencia de los síntomas pero no } \\
\text { disminuyen su recurrencia }\end{array}$ \\
\hline
\end{tabular}

\section{Recomendaciones}

\begin{tabular}{|c|c|}
\hline FUERTE & $\begin{array}{l}\text { No se recomienda el tratamiento hormonal en mujeres con síntomas } \\
\text { vasomotores leves. }\end{array}$ \\
\hline DÉBIL & $\begin{array}{l}\text { En mujeres durante la etapa peri y postmenopausia con síntomas } \\
\text { vasomotores intensos que afecten de manera importante su calidad } \\
\text { de vida, se sugiere ofrecer la posibilidad de realizar tratamiento } \\
\text { hormonal, informando con claridad sobre su efectividad para reducir } \\
\text { los síntomas y sobre todos los riesgos asociados, facilitando que sea } \\
\text { la mujer quien decida con esta información si iniciar el tratamiento. }\end{array}$ \\
\hline FUERTE & $\begin{array}{l}\text { La terapia hormonal está contraindicada en mujeres con antecedentes } \\
\text { de cáncer de mama o de endometrio, enfermedad coronaria, } \\
\text { tromboembolismo, ictus, hepatopatía activa, enfermedad pancreática y } \\
\text { de vesícula biliar o bien con alto riesgo de padecer estas enfermedades. }\end{array}$ \\
\hline FUERTE & $\begin{array}{l}\text { En las mujeres que conservan el útero se recomienda añadir } \\
\text { gestágenos al tratamiento con estrógenos. }\end{array}$ \\
\hline BPC & $\begin{array}{l}\text { El tratamiento hormonal se mantendrá mientras persista la } \\
\text { sintomatología y durante el menor tiempo posible, limitándose a un } \\
\text { periodo máximo de un año. Posteriormente, el tratamiento debería } \\
\text { ser individualizado. Se recomienda iniciar el tratamiento hormonal } \\
\text { con dosis bajas e incrementarlas periódicamente hasta la remisión o } \\
\text { mejora importante de la sintomatología. Una vez alcanzado el efecto } \\
\text { deseado puede intentarse un proceso inverso de disminución gradual } \\
\text { de la dosis y en caso de recidiva de la sintomatología, volver a la } \\
\text { última dosis eficaz. }\end{array}$ \\
\hline BPC & $\begin{array}{l}\text { La elección de la vía y el tipo de tratamiento puede realizarla la } \\
\text { mujer debidamente informada, teniendo en cuenta que, utilizando } \\
\text { dosis bajas o ultrabajas, las pautas secuenciales raramente inducen } \\
\text { sangrado por deprivación. }\end{array}$ \\
\hline
\end{tabular}




\begin{tabular}{|l|l|}
\hline BPC & $\begin{array}{l}\text { El/la profesional puede adaptar las dosis a las necesidades } \\
\text { individuales de la mujer (administrar el tratamiento a intervalos más } \\
\text { largos, dividir comprimidos o parches). }\end{array}$ \\
\hline BPC & $\begin{array}{l}\text { Se recomienda implicar a la mujer en la decisión de iniciar el } \\
\text { tratamiento hormonal. }\end{array}$ \\
\hline
\end{tabular}

\subsubsection{Gestágenos}

Se identificaron cuatro ECAs (392 mujeres) controlados que comparaban el efecto de los gestágenos administrados vía oral o intramuscular en mujeres durante la peri $\mathrm{y}$ postmenopausia con antecedentes de cáncer de mama ${ }^{136} \mathrm{o}$ de cáncer endometrial ${ }^{137}$ frente a placebo $^{132-135}$. Además se identificaron dos ECA (84 mujeres) que compararon los estrógenos con gestágenos en mujeres en la etapa de la postmenopausia. También se identificó una revisión sistemática ${ }^{138}$ (11 ECA, 1 estudio de cohorte y 1 descriptivo), que analizó la eficacia de la progesterona (natural) en mujeres en la etapa de la menopausia y postmenopausia de cualquier edad (excluyendo las que presentaban cáncer de mama). Se evaluó la eficacia del tratamiento en los síntomas asociados a la menopausia, además del efecto sobre la calidad de vida, huesos, sueño, piel, trastornos cognitivos, lípidos plasmáticos y placa de ateroma (riesgo cardiovascular) y los efectos adversos. La mayoría de los estudios utilizaban la vía de administración oral o transdérmica. Las dosis de progesterona se clasificaron como: altas (> $200 \mathrm{mg}$ oral o $>50 \mathrm{mg}$ transdérmica); medias (101-200 mg oral o $21-50 \mathrm{mg}$ transdérmica); o bajas ( $\leq 100 \mathrm{mg}$ oral o $\leq 20 \mathrm{mg}$ transdémica). Uno de los estudios comparaba progesterona frente a medroxiprogesterona, otro frente a progesterona asociada a estrógenos y la mayoría frente a placebo. La duración de los estudios osciló entre 3 meses y 3 años. La RS siguió la metodología PRISMA y no realizó metanálisis de los resultados para ninguna de las variables consideradas, debido a la variabilidad de los estudios incluidos. La calidad de los ECA se evaluó con la metodología Cochrane (sistema GRADE), resultando con calidad baja o muy baja. Se consideraron como principales limitaciones de los ECA el escaso número de pacientes, la ausencia de estandarización de la intervención y el poco seguimiento ${ }^{138}$. Los resultados relativos a los síntomas vasomotores proceden de 5 ECA, pero sólo se dispone de datos numéricos de dos de ellos: uno de progesterona oral ${ }^{139} \mathrm{y}$ otro de progesterona transdérmica.

Adicionalmente se ha identificado una RS reciente sobre gestágenos en mujeres durante la peri y postmenopausia que evaluó la eficacia y seguridad de la progesterona (natural ${ }^{138}$. También se ha identificado un ECA sobre la eficacia de la progesterona (oral) en el tratamiento de los síntomas vasomotores ${ }^{140}$ y un subanálisis posterior, en un subgrupo de mujeres con sofocos moderados-graves ${ }^{141}$. Este ECA doble-ciego de 12 semanas $^{140}$, evaluó la eficacia de progesterona micronizada oral $(300 \mathrm{mg} / \mathrm{d})$ para reducir los síntomas vasomotores (sofocos y sudores nocturnos), frente a placebo, en 133 mujeres sanas de 4462 años, sin factores de riesgo cardiovascular, en la etapa de la postmenopausia. De forma secundaria se analizó el efecto del tratamiento en la función endotelial, como medida del riesgo cardiovascular y se realizó un análisis por subgrupos en función de los síntomas basales. Los principales resultados se presentan como puntuación diaria en la escala de 
síntomas vasomotores, calculada como: frecuencia $\mathrm{x}$ gravedad (sofocos) + frecuencia $\mathrm{x}$ gravedad (sudores nocturnos). También se presentan de forma independiente la frecuencia $y$ la gravedad de los sofocos y los sudores nocturnos. Las limitaciones del estudio y, en particular, la exclusión de mujeres con factores de riesgo cardiovascular reducen la aplicabilidad y generalización de sus resultados. Un subanálisis del ECA anterior, publicado con posterioridad ${ }^{141}$, evaluó la eficacia de la progesterona micronizada oral $(300 \mathrm{mg} / \mathrm{d})$ para reducir la frecuencia de síntomas vasomotores (sofocos y sudores nocturnos), frente a placebo, en un subgrupo de 46 mujeres durante la etapa de la postmenopausia con sofocos moderados-graves (> 50/semana). Adicionalmente, se evaluó el efecto de la suspensión del tratamiento con progesterona en un estudio de cohortes. Además de las limitaciones del ECA original, cabe señalar el escaso número de pacientes analizadas en este subestudio.

Los gestágenos administrados vía oral o intramuscular reducen Calidad baja significativamente los síntomas vasomotores frente a placebo en mujeres en la etapa de la peri y postmenopausia ${ }^{132-135}$ con antecedentes de cáncer de mama ${ }^{136} \mathrm{o}$ de cáncer endometrial ${ }^{137}$.

Los estrógenos frente a gestágenos han mostrado una reducción similar Calidad baja de los síntomas vasomotores en mujeres en la etapa de la postmenopausia ${ }^{142,143}$.

Se dispone de pocos estudios que permitan evaluar e individualizar Calidad baja los efectos secundarios de los gestágenos y se desconoce si el uso de un gestágeno específico influye en el riesgo de cáncer de mama o lo hace en mayor medida que otro ${ }^{131}$.

La progesterona oral $(300 \mathrm{mg} / \mathrm{d})$ se mostró significativamente más eficaz Calidad que placebo para reducir los síntomas vasomotores (escala de frecuencia y muy baja gravedad) en mujeres en la etapa de la postmenopausia, tras 12 semanas de tratamiento (1 ECA; 114 mujeres; DME -28 \%; $p=0,001)^{141}$.

La progesterona micronizada oral $(300 \mathrm{mg} / \mathrm{d})$ se mostró más eficaz Calidad baja que placebo para reducir los síntomas vasomotores (frecuencia y gravedad de los sofocos y sudores nocturnos) en mujeres en la etapa de la postmenopausia sin riesgo cardiovascular, tras 12 semanas de tratamiento, de forma significativa en la escala de síntomas vasomotores (1 ECA, 133 mujeres $)^{140}$ :

* Puntuación (diaria) en la escala de síntomas vasomotores: DME -4,3; IC 95 \% -6,6 a -1,9.

* Frecuencia (diaria) de síntomas vasomotores: DME -1,6; IC 95 \% -2,4 a -0,8.

* Gravedad (diaria) de síntomas vasomotores: DME -0,2; IC 95 \% - 0,4 a -0,0.

La progesterona micronizada oral $(300 \mathrm{mg} / \mathrm{d})$ se mostró más eficaz Calidad que placebo para reducir la frecuencia diaria de síntomas vasomotores muy baja (sofocos y sudores nocturnos) en mujeres en la etapa de la postmenopausia sin riesgo cardiovascular, con síntomas moderados-graves, tras 12 semanas de tratamiento (1 ECA, 46 mujeres, 12 semanas; DME -0,2; IC $95 \%$-3,5 a - -0,4) $)^{141}$. 
La progesterona transdérmica $(60 \mathrm{mg} / \mathrm{d})$ se mostró más eficaz que Calidad baja placebo para reducir la frecuencia semanal de sofocos y sudores nocturnos en 68 mujeres en la etapa de la postmenopausia tras 24 semanas de tratamiento (1 ECA; 68 mujeres; DM -8 ; IC $95 \%$-17 a 1; p = 0,07) $)^{144}$.

La progesterona transdérmica $(40 \mathrm{mg} / \mathrm{d})$ se mostró más eficaz que Calidad baja placebo para reducir los síntomas vasomotores en 84 mujeres en la etapa de la postmenopausia tras 24 semanas de tratamiento (1 ECA; 84 mujeres; DM -0,6; IC $95 \%-1,2$ a 0,$0 ; p=0,06)^{144}$.

\section{Resumen de la evidencia -gestágenos}

\begin{tabular}{|l|l|}
\hline & Los gestágenos, vía oral, intramuscular o transdérmica, parecen \\
Calidad & reducir los síntomas vasomotores en mujeres en la etapa de la \\
BAJA & $\begin{array}{l}\text { postmenopausia }{ }^{132,137,138,140,142,143} \text {. La seguridad de su empleo en } \\
\text { mujeres con cáncer de mama no ha sido totalmente establecida }\end{array}$ \\
\hline
\end{tabular}

\section{Recomendaciones}

\begin{tabular}{|l|l|}
\hline DÉBIL & $\begin{array}{l}\text { En mujeres en la etapa de la peri y postmenopausia con contraindicaciones } \\
\text { en el uso de estrógenos y síntomas vasomotores intensos, se sugiere } \\
\text { valorar la utilización de los gestágenos por vía oral o intramuscular, } \\
\text { individualizando el balance beneficio/riesgo para el tratamiento de los } \\
\text { síntomas vasomotores. }\end{array}$ \\
\hline
\end{tabular}

\subsubsection{Andrógenos}

Se ha identificado una $\mathrm{RS}^{145}$ y dos $\mathrm{ECAs}^{146,147}$ que analizan la efectividad de los andrógenos como tratamiento de los síntomas vasomotores en mujeres durante la etapa de la peri y postmenopausia . La revisión sistemática Cochrane de Somboonporn et al., de $2005^{145}$ incluyó 35 ECA (4.768 mujeres) que evaluaban el tratamiento con testosterona en mujeres durante la etapa de la peri y postmenopausia. Solamente dos de los estudios fueron incluidos en un metánalisis sobre síntomas vasomotores.

Uno de los $\mathrm{ECA}^{146}$, que incluía 71 mujeres sin patología en la etapa de la postmenopausia, comparaba los tratamientos orales con: Estratab (estrógeno), Estratab y Prometrium (gestágeno micronizado), Estratab y Provera (gestágeno sintético), Estratest (estrógeno y metiltestosterona) o placebo.

El otro $\mathrm{ECA}^{147}$, que incluyó a 95 mujeres con menopausia postquirúrgica y disminución del deseo sexual, comparaba el tratamiento oral con estrógenos (estrógeno esterificado) frente al tratamiento oral con estrógenos más testosterona (estrógeno esterificado y metiltestosterona). 
Un metanálisis de los dos ECA (que incluyó 166 mujeres) no mostró Calidad diferencias en la media de síntomas vasomotores en las mujeres tratadas muy baja con terapia hormonal o con terapia hormonal y testosterona (diferencia de medias 0,09; IC $95 \%-0,18$ a 0,6).

El tratamiento hormonal combinado con testosterona está asociado a Calidad un aumento en la incidencia de crecimiento del vello y de acné y a una muy baja disminución de las lipoproteínas de alta densidad (HDL). Estos efectos adversos pueden ser diferentes según la dosis y la vía de administración de la testosterona. No hay suficiente evidencia para determinar los efectos de la testosterona a largo plazo $^{145}$.

La North American Menopause Society publicó en $2005^{131}$ una serie de recomendaciones sobre el papel de la testosterona exógena en las mujeres en la etapa de la postmenopausia. No encontró datos suficientes para recomendar el uso de testosterona para reducir los sofocos.

\section{Resumen de la evidencia - andrógenos}

\begin{tabular}{|c|l|}
\hline Calidad & $\begin{array}{l}\text { Los andrógenos no han mostrado resultados consistentes en el } \\
\text { tratamiento de los síntomas vasomotores }\end{array}$ \\
MUY A A corto plazo se ha \\
observado un aumento en la incidencia de vello y acné y una \\
disminución de las lipoproteínas de alta densidad (HDL). Se \\
desconocen sus efectos a largo plazo
\end{tabular}

\section{Recomendaciones}

\begin{tabular}{|l|l|}
\hline DÉBIL & $\begin{array}{l}\text { Se recomienda no utilizar el tratamiento con andrógenos para tratar } \\
\text { los síntomas vasomotores en mujeres durante la etapa de la peri y } \\
\text { postmenopausia. }\end{array}$ \\
\hline
\end{tabular}

\subsubsection{Tibolona}

Los resultados de dos $\mathrm{RS}^{148,149}$ y posteriores $\mathrm{ECA}^{150-154}$ (13 ECA, Calidad baja 1.749 mujeres) muestran que la tibolona, comparada con el placebo, reduce de forma significativa los sofocos y las sudoraciones nocturnas. La eficacia de la tibolona es similar a la de los estrógenos con o sin gestágenos ${ }^{155-157}$. La dosis de $2,5 \mathrm{mg} /$ día de tibolona es la óptima y ha sido la más utilizada en la mayoría de los $\mathrm{ECA}^{155,156}$.

El ECA de Somunkiran et al., de $2007^{152}$ incluyó un total de 40 Calidad mujeres con menopausia postquirúrgica y comparó, con un diseño muy baja cruzado, el tratamiento con tibolona frente al tratamiento con 17betaestradiol durante 6 meses. Ambos tratamientos mostraron mejoras comparables en la disminución de los síntomas vasomotores. 
Los diferentes ECA no muestran a corto plazo efectos adversos Calidad baja importantes. Se ha descrito un aumento del riesgo de sangrados irregulares y una disminución de las lipoproteínas de alta densidad. Algunos estudios muestran efectos adversos androgénicos (hirsutismo, piel grasa), pero sin mostrar resultados consistentes ${ }^{155,156}$.

Un estudio de cohortes mostró un incremento significativo de la Calidad incidencia de cáncer de mama en el grupo de mujeres que recibían muy baja tibolona (RR 1,45; IC 95 \% 1,25-1,68) ${ }^{148}$.

La tibolona resultó más eficaz que el placebo para aliviar los Calidad síntomas vasomotores en mujeres en etapa de la postmenopausia (2 moderada ECAs, $\mathrm{n}=847$; OR 0,42; IC $95 \% 0,25$ a 0,69), siendo la reducción significativa con dosis de $2,5 \mathrm{mg} /$ día de tibolona ${ }^{158}$.

La tibolona ha resultado menos eficaz que la THS, a dosis equipotentes, Calidad para reducir la frecuencia de los síntomas vasomotores en mujeres en etapa moderada de la postmenopausia (2 ECAs, $\mathrm{n}=545$; OR 4,16; IC $95 \%$ 1,50 a 11,58) ${ }^{158}$.

La presencia de sangrado vaginal irregular fue más frecuente con Calidad tibolona que con placebo ( 7 ECAs; $n=7.462$; OR 2,75; IC $95 \% 1,99$ a moderada $3,80)^{158}$.

La incidencia de sangrado vaginal irregular fue menor con la tibolona Calidad que la observada frente a TSH (estrógenos junto a gestágenos) (15 ECAs, moderada $\mathrm{n}=6.342$; OR 0,32 ; IC $95 \% 0,24$ a 0,42$)^{158}$.

Se observó un aumento del riesgo de accidente cerebrovascular en Calidad mujeres mayores de 60 años en el seguimiento a largo plazo (un ECA; moderada 3.098 mujeres; OR 2,18; IC $95 \%$ 1,12 a 4,21 $)^{158}$.

No hubo evidencia de diferencias de seguridad a largo plazo entre Calidad tibolona y terapia hormonal sustitutiva combinada ${ }^{158}$.

moderada

En cuanto a su seguridad a largo plazo, un ECA con 3.098 mujeres con Calidad cáncer de mama fue interrumpido prematuramente debido a un aumento del moderada riesgo de recurrencia tumoral (OR 1,50; IC $95 \% 1,21$ a 1,85); sin embargo otro ECA se asoció con una reducción del cáncer de mama frente a placebo (OR 0,32; IC $95 \%$ 0,13 a 0,79). No se detectó evidencia clara del efecto de la tibolona sobre el cáncer de endometrio con respecto a placebo.

La tibolona, a dosis de 2,5 mg/día durante 12 semanas, comparada Calidad frente a placebo, redujo de forma significativa el número diario de sofocos muy baja (1 ECA; 485 mujeres; -8,21 vs. -5,82, respectivamente; $\mathrm{p}<0,001$ ). Del mismo modo, la tibolona redujo la intensidad de los sofocos (Diferencia de medias ajustada 1,14; error estandar 0,$07 ; \mathrm{p}<0,001)^{159}$.

Los pacientes tratados con tibolona presentan mayor frecuencia de Calidad sangrado vaginal irregular en comparación con placebo (1 ECA; $23 \%$ vs. muy baja $9 \% ; \mathrm{p}<0,001)^{159}$. 


\section{Resumen de la evidencia - tibolona}

\begin{tabular}{|c|c|}
\hline $\begin{array}{l}\text { Calidad } \\
\text { MODERADA } \\
\text { /BAJA }\end{array}$ & $\begin{array}{l}\text { La tibolona se ha mostrado superior al placebo para el tratamiento } \\
\text { de los síntomas vasomotores }{ }^{150,152,155,156,158,159} \text {. Los estudios disponibles } \\
\text { sugieren una eficacia inferior }{ }^{158} \text { o similar a la del tratamiento } \\
\text { hormonal }^{155-157} \text {. No obstante, la información disponible sobre la } \\
\text { tibolona es todavía escasa y de peor calidad que la disponible sobre } \\
\text { el tratamiento hormonal. }\end{array}$ \\
\hline $\begin{array}{c}\text { Calidad } \\
\text { MODERADA }\end{array}$ & $\begin{array}{l}\text { La tibolona se ha relacionado con un aumento indeseable en la } \\
\text { frecuencia de sangrados vaginales irregulares en relación al placebo, } \\
\text { pero con una menor frecuencia de sangrados en relación a la THS }{ }^{158} \text {. }\end{array}$ \\
\hline $\begin{array}{l}\text { Calidad } \\
\text { MODERADA } \\
\text { /MUY BAJA }\end{array}$ & $\begin{array}{l}\text { Los resultados sobre la relación de la tibolona y el riesgo de cáncer de } \\
\text { mama no son concluyentes }{ }^{158,160} \text {, aunque se ha detectado mayor índice } \\
\text { de recurrencia en mujeres que habían sufrido cáncer de mama }{ }^{158} \text {. }\end{array}$ \\
\hline $\begin{array}{l}\text { Calidad } \\
\text { MODERADA }\end{array}$ & $\begin{array}{l}\text { La tibolona puede aumentar el riesgo de accidente cerebrovascular, } \\
\text { especialmente en mujeres mayores de } 60 \text { años }^{158} \text {. }\end{array}$ \\
\hline
\end{tabular}

\section{Recomendaciones}

\begin{tabular}{|c|l|}
\hline \multirow{3}{*}{ DÉBIL } & $\begin{array}{l}\text { En mujeres en la etapa de la postmenopausia con síntomas } \\
\text { vasomotores intensos que afecten su calidad de vida en las que el } \\
\text { tratamiento hormonal con estrógenos no haya sido efectivo o no se } \\
\text { tolere, se puede valorar iniciar el tratamiento con tibolona, teniendo } \\
\text { en cuenta el riesgo/beneficio de esta intervención. }\end{array}$ \\
\hline \multirow{3}{\text{BPC}}{} & $\begin{array}{l}\text { En las mujeres en las que sea necesario iniciar el tratamiento con } \\
\text { tibolona durante la etapa de la postmenopausia, se sugiere } \\
\text { administrarla después del primer año de menopausia. Si el } \\
\text { tratamiento se inicia antes, aumentan las posibilidades de sangrado } \\
\text { vaginal irregular o manchado. }\end{array}$ \\
\hline
\end{tabular}




\subsubsection{Tratamientos no hormonales}

\subsubsection{Antidepresivos}

Se han identificado 3 RS y 27 ECA que evalúan la eficacia de los antidepresivos para los síntomas vasomotores en mujeres durante la peri y postmenopausia. No se ha identificado ningún ECA que evalúe la eficacia de bupropion, desipramina, fluvoxamina o trazodona para el tratamiento de los síntomas vasomotores en mujeres en la etapa de la peri y postmenopausia.

Además se han identificado cinco estudios recientes: una RS con metanálisis de inhibidores selectivos de la recaptación de serotonina (ISRS) ${ }^{161}$ y cuatro ECAs sobre desvenlafaxina ${ }^{159,162-164}$.

\subsection{ISRS}

La $\mathrm{RS}^{161}$ identificada analizó la eficacia de los ISRS (paroxetina, escitalopram, citalopram y sertralina) para aliviar los síntomas vasomotores, frente a placebo, en mujeres sanas durante la peri y la postmenopausia reciente. En total incluyó 11 ECA con un seguimiento de 1-9 meses y 2.069 mujeres, con un rango de edad entre 36 a 76 años, un tiempo medio desde la menopausia de 2,3-6,6 años, sin depresión ni cáncer, ni tratamiento con moduladores selectivos de los receptores estrogénicos o SERM (tamoxifeno, raloxifeno) ni terapia hormonal sustitutiva. Puesto que no todos los ECA tenían la misma duración, para el metanálisis se seleccionaron los resultados a la $6^{\mathrm{a}} \mathrm{y}$ $8^{\mathrm{a}}$ semanas de tratamiento.

Los ISRS analizados mostraron una disminución de la frecuencia de los Calidad baja sofocos estadísticamente significativa frente a placebo, aunque modesta (diferencia media ponderada de $-0,93$; IC $95 \%-1,46$ a $-0,37 ; \mathrm{I} 2=21 \%$ ) $^{161}$.

La reducción de la intensidad o gravedad de los sofocos fue significativa Calidad baja con los ISRS frente a placebo, aunque heterogénea (diferencia de medias estandarizada de $-0,34$; IC $95 \%-0,59$ a $-0,10 ;$ I2 $=47 \%)^{161}$.

En cuanto a su seguridad, los efectos adversos de los ISRS cuando se Calidad baja utilizaron para tratar los sofocos fueron leves y aunque fueron más frecuentes, no difirieron significativamente con respecto al placebo ${ }^{161}$.

\subsection{Paroxetina}

Se identificaron una $\mathrm{RS}^{165}$ y $5 \mathrm{ECAs}^{166-170}$ que analizaron la eficacia de la paroxetina como tratamiento para los síntomas vasomotores en mujeres durante la peri y postmenopausia.

La paroxetina a dosis de $10 \mathrm{mg}$ a $25 \mathrm{mg}$ redujo la frecuencia de sofocos Calidad diarios en -1,66 (IC $95 \%-2,43$ a $-0,89$ ) y la intensidad de los mismos en un moderada $30 \%$ (IC $95 \% 13 \%$ al $41 \%$ ) (2 ECA, 316 mujeres) ${ }^{165}$. No se observaron diferencias significativas entre las distintas dosis de paroxetina (de $10 \mathrm{mg}$ a $25 \mathrm{mg}$ ) y la reducción de sofocos diarios y su intensidad. 
En mujeres sintomáticas después de la interrupción de tratamiento Calidad hormonal, la paroxetina de liberación controlada $(12,5 \mathrm{mg}-25 \mathrm{mg})$, moderada comparada con placebo ha resultado efectiva (1 ECA; 64 mujeres) ${ }^{166}$.

La paroxetina, a dosis de 7,5 mg/día, comparada con placebo, redujo Calidad baja la frecuencia de los sofocos en $-0,97$ (IC $95 \%-1,79$ a $-0,15$ ) y en $-1,46$ (IC $95 \%-2,32$ a -0,60) (1 RS; 2 ECA; 1.174 mujeres) ${ }^{161}$.

La paroxetina a dosis de $7,5 \mathrm{mg}$, comparada con placebo, redujo la Calidad baja media semanal de sofocos al mes y a los tres meses del inicio del tratamiento (2 ECAs, 591 mujeres, valores acumulados no proporcionados, $\mathrm{p}<0,0001$ ) así como la intensidad de los mismos en ambos periodos de seguimiento (2 ECAs, 591 mujeres, valores acumulados no proporcionados, $\mathrm{p}=0,0006 \mathrm{y}$ $\mathrm{p}=0,0110$, respectivamente) ${ }^{170}$.

En comparación con otros ISRS, los resultados de eficacia comparada Calidad baja del metanálisis no muestran que paroxetina sea significativamente más eficaz para el tratamiento de los síntomas vasomotores frente a sertralina $(-0,40$; IC $95 \%-3.11$ a 2,46), fluoxetina (-1,09; IC $95 \%-2,94$ a 0,70$)$, escitalopram $\left(0,82 ;\right.$ IC $95 \%-2,06$ a 3,82) y citalopram $\left(-0,69 ;\right.$ IC $95 \%-2,47$ a 1,14) ${ }^{161}$.

Aunque no existen datos concluyentes entre los estudios evaluados, Calidad se ha observado una mayor incidencia de náuseas al aumentar la dosis de moderada paroxetina. En comparación con placebo, la paroxetina a $7,5 \mathrm{mg}$ ha mostrado un mayor riesgo en la aparición de náuseas (paroxetina 3,8 \% $v s$. placebo $1,4 \%)$, fatiga $(3,4 \% v s$. placebo $1,5 \%)$ y mareos $(2,0 \% v s$. placebo $0,8 \%)^{170}$. El riesgo de abandono del tratamiento con paroxetina es superior al del abandono del tratamiento con placebo (1 ECA; 165 mujeres; RR 3,08; IC $95 \%$ 0,71 a 13,30) ${ }^{168}$. Asimismo, la paroxetina se ha relacionado con un aumento del riesgo de mortalidad en mujeres con cáncer de mama en tratamiento con tamoxifeno ${ }^{169}$, mientras que otros antidepresivos como fluoxetina, sertralina, venlafaxina o citalopram no han presentado un aumento significativo de este riesgo.

\subsection{Fluoxetina}

Se identificaron dos RS/metanálisis ${ }^{161,165}$ que analizaban la eficacia de la fluoxetina para el tratamiento de los síntomas vasomotores en mujeres durante la etapa de la peri y postmenopausia.

Tanto en mujeres con y sin historia de cáncer de mama, la fluoxetina Calidad baja a dosis de $20 \mathrm{mg} / \mathrm{d}$ no ha mostrado una reducción significativa en la frecuencia de sofocos en comparación con placebo ${ }^{165}$.

En comparación con otros ISRS, la fluoxetina no ha mostrado ser significativamente más eficaz para el tratamiento de los síntomas vasomotores comparada con paroxetina $(-1,09$; IC $95 \%-2,94$ a 0,70$)$; sertralina (-0,69; IC $95 \%-3,64$ a 2,12); citalopram (0,40; IC $95 \%-1,02$ a $1,92)$ y escitalopram $(1,914 ; \text { IC } 95 \%-1,11 \text { a } 5,06)^{161}$. 
En mujeres sin historia de cáncer de mama, el aumento de la dosis a Calidad baja $30 \mathrm{mg}$ no mostró beneficio en la reducción de $\operatorname{sofocos}^{171}$, aunque redujo la intensidad de los mismos en un $24 \%$ en comparación con placebo $(1 \mathrm{ECA}, \mathrm{p}=0,2)^{172}$.

No se han observado diferencias significativas, en cuanto a efectos adversos, entre mujeres en tratamiento con fluoxetina y placebo ${ }^{172}$. El riesgo de abandono del tratamiento fue similar con la fluoxetina que con el placebo (1 ECA, 100 mujeres, RR 0,95; IC 95 \% 0,58 a 1,55).

\subsection{Sertralina}

Se identificó un metanálisis que analizó 3 ECA, con un total de 230 mujeres con y sin historia de cáncer de mama ${ }^{173}$ y un ECA posterior en mujeres con cáncer de mama o con riesgo de desarrollarlo ${ }^{174}$. Además, se identificó una RS con tres ECA sobre sertralina, a dosis de $50-100 \mathrm{mg} /$ día, en 219 mujeres ${ }^{161}$. Dos de estos $\mathrm{ECA}^{175,176}$ ya habían sido incluidos en otra $\mathrm{RS}^{173}$. El tercer ECA incluido en esta nueva RS no evaluó la eficacia de sertralina en la frecuencia e intensidad de los sofocos ${ }^{161}$.

La sertralina, a dosis de $50 \mathrm{mg} / \mathrm{d}$ a $100 \mathrm{mg} / \mathrm{d}$, no redujo significativamente Calidad baja la frecuencia de sofocos frente a placebo (1 RS con metanálisis; 4 ECAs; 230 mujeres) ${ }^{173,174}$.

La sertralina, a dosis de 50-100 mg/día, no redujo significativamente Calidad baja la frecuencia diaria de los sofocos, frente a placebo (diferencia media ponderada de -4,66; IC $95 \%-17,94$ a 8,62 y $-0,70$; IC $95 \%-2,78$ a 1,38) (3 ECAs, 219 mujeres ${ }^{161,175,176}$.

En comparación con otros ISRS, sertralina no demostró ser significativamente más eficaz en el tratamiento de los síntomas vasomotores que paroxetina $(-0,40$; IC $95 \%-3,11$ a 2,46$)$; fluoxetina $(-0,69$; IC $95 \%-3,64$ a 2,12); escitalopram (1,225; IC $95 \%-2,44$ a 4,89) y citalopram (-0,29; IC $95 \%-3,32$ a 2,57) ${ }^{161}$.

\subsubsection{Venlafaxina}

La efectividad de la venlafaxina para la mejoría de los sofocos se ha evaluado mayoritariamente en mujeres con historia de cáncer de mama en tratamiento con terapia antiestrógena. Se identificó una RS, que analizó 2 ECA con 247 mujeres sobre el efecto de venlafaxina para la reducción de la frecuencia de los sofocos ${ }^{165}, 1 \mathrm{RS}$ (1 ECA) sobre el efecto de venlafaxina a dosis de $75 \mathrm{mg}$ en la reducción de la intensidad de los $\operatorname{sofocos}^{173}$, en comparación con placebo y tres $\mathrm{ECA}^{177-179}$. Se identificaron también estudios que analizaron los efectos adversos de venlafaxina frente a placebo ${ }^{180,181}$ y que comparaban la eficacia de venlafaxina y el riesgo de abandono del tratamiento frente a la de la clonidina ${ }^{177,178}$.

La venlafaxina no mostró efecto en la reducción de la frecuencia de Calidad sofocos $(-0,49 \text { sofocos diarios, IC } 95 \%-2,40 \text { a } 1,41)^{165}$, pero sí mostró un moderada efecto moderado en la reducción de la intensidad de los sofocos (33\%), en comparación con placebo ${ }^{173,180}$. Los otros tres ECA no mostraron resultados consistentes ${ }^{177-179}$. 
En comparación con placebo, la venlafaxina se asoció a la aparición Calidad de forma significativa de sequedad de boca, estreñimiento, náuseas y moderada disminución del apetito ${ }^{180,181}$. Dos ECA compararon la eficacia de la venlafaxina con la de la clonidina y mostraron un riesgo de abandono del tratamiento mayor al del abandono del tratamiento con clonidina (2 ECA, 17 eventos de 70 mujeres, RR 1,89; IC $95 \% 0,90$ a 3,96) $)^{177,178}$.

\subsubsection{Desvenlafaxina}

Se ha identificado una RS (6 ECAs, 1.931 mujeres) ${ }^{182}$ que evaluó la eficacia y seguridad de la administración de desvenlafaxina para reducir la frecuencia y severidad de los sofocos en mujeres durante la etapa de la postmenopausia.

La desvenlafaxina a una dosis de $100 \mathrm{mg}$ redujo de forma significativa Calidad baja la frecuencia de sofocos en comparación con placebo a la semana 12 de tratamiento (6 ECAs, 1.167 mujeres, diferencia de medias estandarizadaDME $=-0,49$; IC $95 \%-0,91$ a $-0,07, p=0,003)$ y en las 26 semanas de tratamiento (3 ECAs, 803 mujeres, DME $=-0,74$, IC $95 \%-1,05$ a $-0,44)^{182}$.

Desvenlafaxina a una dosis de $100 \mathrm{mg}$ redujo la puntuación total a la Calidad baja Greene Climacteric Scale en comparación con placebo (3 ECAs, 794 mujeres, DM $=-0,40$, IC $95 \%-0,70$ a $-0,10)^{182}$.

Desvenlafaxina a dosis de $150 \mathrm{mg}$ redujo el número medio de Calidad baja despertares nocturnos en comparación con placebo (3 ECAs, 749 mujeres, $\mathrm{DM}=-0,56$, IC $95 \%-0,80$ a $-0,32)^{182}$.

La administración de desvenlafaxina a $100 \mathrm{mg}$ incrementó el riesgo de Calidad baja efectos adversos (nauseas, estreñimiento, diarrea, boca seca, hipertensión, somolencia, astenia, mareo, insominio, etc.) (2 ECAs, 940 mujeres, RR 1,22; IC $95 \%$ 1,08-1,38) y de discontinuidad del tratamiento en comparación con placebo (2 ECAs, 940 mujeres, RR 1,68, IC 95 \% 1,26-2,24) ${ }^{182}$.

\subsubsection{Citalopram}

Una reciente RS con metanálisis ${ }^{161}$ incluía tres ECA (2.069 mujeres durante la menopausia y postmenopausia) para evaluar la eficacia de citalopram frente a placebo ( \pm fluoxetina o THS) en el tratamiento de los síntomas climatéricos (frecuencia e intensidad de sofocos). Un ECA de publicación reciente comparó distintas dosis de citalopram $(10,20$ y $30 \mathrm{mg} /$ día $)$ frente a placebo $^{183}$.

En relación a la frecuencia de sofocos, en dos ECAs no se Calidad baja observaron diferencias significativas entre placebo y citalopram $(-0,79$ [IC $95 \%-1,82$ a 0,24 ] y 1,20 [IC $95 \%-0,49$ a 2,89]). En cuanto a las puntuaciones de intensidad de los sofocos, dos ECAs ofrecían resultados, pero sólo uno mostró reducciones significativas en términos de diferencia media ponderada (-1,09 [IC $95 \%-1,69$ a $-0,49])$.

Citalopram, a cualquiera de las dosis comparadas, fue más eficaz que Calidad placebo en relación a la disminución en la puntuación de intensidad de los moderada 
sofocos y en la frecuencia de los mismos, siendo las diferencias estadísticamente significativas en ambas medidas de resultado ${ }^{183}$.

En comparación con otros ISRS, citalopram no ha mostrado ser Calidad baja significativamente más eficaz en el tratamiento de los síntomas vasomotores que fluoxetina $(0,40$; IC $95 \%-1,02$ a 1,92), sertralina $(-0,29$; IC $95 \%-3,32$ a 2,57), paroxetina $(-0,69 ;$ IC $95 \%-2,47$ a 1,14$)$ y escitalopram (1,511; IC $95 \%-1,55$ a 4,58$)^{161}$.

\subsection{Escitalopram}

En una $\mathrm{RS}^{161}$ con metanálisis que analizaba la eficacia de los ISRS para tratar los síntomas vasomotores en mujeres durante la etapa de la menopausia y postmenopausia se incluyeron dos ECA $(\mathrm{n}=231)$, de 8 semanas de duración en los que se comparó escitalopram, a dosis de 10-20 mg/día, frente a placebo.

Escitalopram no mostró diferencias significativas frente a placebo en Calidad baja la disminución de la frecuencia de los sofocos (Diferencia media ponderada [DMP]: -4,50; IC $95 \%-9,63$ a 0,63; DMP: -1,18; IC $95 \%-4,01$ a 1,65). Tampoco ha mostrado diferencias significativas en relación a la intensidad de los sofocos frente a placebo (Diferencia de medias estandarizada [DME]: -0,24; IC $95 \%-0,52$ a 0,05).

En comparación con otros ISRS, escitalopram se mostró más eficaz para el tratamiento de los síntomas vasomotores, aunque sin alcanzar significación estadística, que citalopram (1,511; IC $95 \%$; $-1,55$ a 4,58), fluoxetina (1,914, IC $95 \%-1,11$ a 5,06), sertralina $(1,225$, IC $95 \%-2,44$ a $4,89)$ y paroxetina $(0,82 \text {, IC } 95 \%-2,06 \text { a } 3,82)^{161}$.

\subsubsection{Otros fármacos}

\subsection{Clonidina}

Se identificó una $\mathrm{RS}^{165}$, que incluía también mujeres en tratamiento con terapia antiestrogénica, y 2 ECAs ${ }^{177,178}$ que analizaban la eficacia de la clonidina como tratamiento de los síntomas vasomotores en mujeres durante la etapa de la menopausia y postmenopausia.

La clonidina disminuyó la frecuencia de sofocos por día (-0,95, IC 95 \% Calidad baja -1,44 a -0,47) en comparación con placebo (4 ECA, 444 mujeres) ${ }^{165}$.

En comparación con la venlafaxina (140 mujeres en mujeres con cáncer de mama), la clonidina redujo la intensidad de los sofocos entre un $55 \%$ y un $57 \%$, y la venlafaxina entre un $39 \%$ y $49 \%$, sin diferencias significativas entre ambas ${ }^{177,178}$. Un ECA mostró que la calidad del sueño y el deseo sexual mejoraban a partir de la $8^{\text {a }}$ semana de tratamiento, aunque sin cambios en el estado de ánimo ${ }^{177}$. 
El riesgo de abandono del tratamiento con venlafaxina se ha mostrado mayor al del abandono del tratamiento con clonidina (2 ECA, 17 eventos de 70 mujeres, RR 1,89; IC $95 \%$ 0,90-3,96). Entre los efectos adversos de la clonidina se han descrito sequedad de boca, somnolencia, fatiga o náuseas ${ }^{178}$. La evidencia sobre estos efectos adversos, tanto a medio como a largo plazo, es insuficiente.

\subsection{Gabapentina}

Se identificó una RS reciente, que empleó 3 ECA $(n=173)$ para el análisis conjunto de los datos ${ }^{184}$, un metanálisis ( $\left.3 \mathrm{ECA}, \mathrm{n}=450\right)^{173}$; ambos incluyendo mujeres con y sin historia de cáncer de mama, y 1 ECA que analizaba la eficacia de la gabapentina comparada con estradiol ${ }^{184}$. En la mayoría de los estudios, la dosis de gabapentina se ha dado de forma progresiva a las mujeres, hasta llegar a la dosis clínica de tratamiento requerida según la intervención. Además se identificó un estudio de cohortes que analizaba la seguridad de este fármaco ${ }^{185}$.

Otra $\mathrm{RS}^{184}$ incluyó 7 EC (abiertos, no controlados) y ECA relizados en mujeres con menopausia (natural o inducida por tamoxifeno) con y sin cáncer de mama, y hombres con historial de cáncer de próstata sometidos a deprivación androgénica $(\mathrm{n}=901)$. Los pacientes incluidos podían estar en tratamiento con otros medicamentos como terapias endocrinas y antidepresivos. La gabapentina se administró a dosis de 900-2.400 mg/día y los periodos de ajuste tuvieron una duración de 3-12 días. En el metanálisis sólo se incluyeron 4 ECAs que analizaban la eficacia de gabapentina frente a placebo en mujeres. De ellos, se disponía de datos sólo de 3 ECA que se emplearon para el análisis de datos agrupados de eficacia en la frecuencia y la intensidad de los sofocos.

La gabapentina redujo el número de sofocos en un $26 \%$ (IC $95 \%$ de Calidad $10 \%$ a $40 \%$ ) y, a dosis entre $900 \mathrm{mg} /$ día y $2.400 \mathrm{mg} /$ día, redujo la moderada frecuencia y la intensidad de $\operatorname{los} \operatorname{sofocos}^{173}$ frente a placebo.

La gabapentina redujo tanto la frecuencia (diferencia media ponderada Calidad frente a placebo $=23,72$ [IC $95 \%$ 16,46-30,97]; $\mathrm{p}<0,001$ ) como la intensidad muy baja de los sofocos (diferencia media ponderada frente a placebo $=27,26$ [IC $95 \%$ 21,24-33,29]; p < 0,001); aunque se constató una heterogeneidad significativa entre los estudios $(I 2=97,8 \% \text { y } 95,6 \% \text {, respectivamente })^{184}$.

No se han observado diferencias entre el tratamiento con gabapentina Calidad a dosis de $900 \mathrm{mg} / \mathrm{d}$ o el tratamiento combinado con antidepresivos, ni en la moderada disminución de la frecuencia ni la intensidad de los sofocos ${ }^{186}$.

Atendiendo a la vía de administración, se comparó gabapentina en Calidad comprimidos $(600 \mathrm{mg} / \mathrm{d})$ frente a parche semanal de estradiol muy baja transdérmico a bajas dosis $(25 \mathrm{mcg} / \mathrm{d})^{184}$. Tras 8 semanas, la reducción de la frecuencia e intensidad de los sofocos disminuyó en ambos grupos, respecto a los valores basales, aunque no hubo diferencias significativas de gabapentina frente a estradiol (frecuencia: $58,9 \%$ vs. $70,1 \%$ e intensidad: $60,6 \%$ vs. 68,2 \%, respectivamente, $\mathrm{p}>0,05)(\mathrm{n}=45)$. 
Gabapentina se asoció a efectos adversos como somnolencia, vértigos y fatiga y estradiol a mastodinia, manchado vaginal y reacciones alérgicas locales. El cumplimiento fue superior en el grupo de gabapentina $(95,6 \%)$ que en el grupo de estradiol $(90,9 \%)$.

En los resultados agrupados de seguridad, los abandonos por efectos Calidad adversos fueron más frecuentes con gabapentina que con placebo muy baja (RR 2,09 [IC $95 \%$ 1,13-3,85]; $\mathrm{p}=$ 0,02). El riesgo de efectos adversos, por síntomas agrupados, fue significativamente mayor con gabapentina que con placebo (fatiga/somnolencia: RR 4,78 [IC $95 \%$ 2,23-10,25]; $\mathrm{p}<0,001$; I2 $=0 \%$; y mareos/inestabilidad: RR 6,94 [IC $95 \% 3,19-15,13$ ]; $\mathrm{p}<0,001$; $\mathrm{I} 2=63,1 \%)$.

Un estudio de cohortes reciente ha mostrado un riesgo aumentado de Calidad suicidio asociado con gabapentina, así como con otros antiepilépticos moderada (lamotrigina, oxcarbazepina, tiagabina y valproato) en comparación con topiramato ${ }^{185}$.

\subsection{Metildopa}

No se han identificado ECAs recientes con metildopa para el tratamiento de los síntomas vasomotores. Se dispone de algunos ECA de la década de los años ochenta que mostraban una discreta eficacia de la metildopa ${ }^{187-189}$.

La metildopa se asocia a efectos adversos como somnolencia, Calidad sequedad de boca, hipotensión, pero no se dispone de evidencia al muy baja respecto ni a corto ni a largo plazo.

Resumen de la evidencia - antidepresivos y otros fármacos

\begin{tabular}{|c|c|}
\hline \multicolumn{2}{|l|}{ Antidepresivos } \\
\hline $\begin{array}{c}\text { Calidad } \\
\text { MODERADA } \\
\text { /BAJA } \\
\text { /MUY BAJA }\end{array}$ & $\begin{array}{l}\text { Los antidepresivos han mostrado resultados inconsistentes y } \\
\text { moderados en el tratamiento de los sofocos. }\end{array}$ \\
\hline \multicolumn{2}{|r|}{ Inhibidores Selectivos de la Recaptación de Serotonina (ISRS) } \\
\hline $\begin{array}{c}\text { Calidad } \\
\text { MODERADA } \\
\text { /BAJA } \\
\text { /MUY BAJA }\end{array}$ & $\begin{array}{l}\text { La paroxetina a dosis de } 7,5 \mathrm{mg} \text { a } 25 \mathrm{mg} \text { se ha mostrado efectiva } \\
\text { para la reducción de los sofocos y su intensidad }{ }^{161,165,166} \text {. La fluoxetina } \\
\text { no ha mostrado beneficio en la reducción de la frecuencia de los } \\
\text { sofocos }{ }^{161,165,172} \text {, aunque parece tener un efecto discreto en la } \\
\text { intensidad de los mismos a dosis superiores }(30 \mathrm{mg}) \text {, al igual que } \\
\text { venlafaxina }{ }^{165,173,178-181161} \text { y citalopram } \\
\text { La sertralina y el escitalopram no han mostrado beneficio en la } \\
\text { reducción de los sofocos }{ }^{173,174,176161,191} \text {. }\end{array}$ \\
\hline
\end{tabular}




\begin{tabular}{|c|c|}
\hline & $\begin{array}{l}\text { La desvenlafaxina (a dosis de } 100 \text { y } 150 \mathrm{mg} \text { ) se ha mostrado efectiva } \\
\text { en la reducción de la frecuencia e intensidad (medida con la Greene } \\
\text { Climacteric Scale) de los sofocos y en el número de despertares } \\
\text { nocturnos }^{182} \text {. } \\
\text { Los antidepresivos pueden presentar efectos adversos como náuseas, } \\
\text { sequedad de boca, estreñimiento, insomnio, disfunción sexual y } \\
\text { disminución del apetito }\end{array}$ \\
\hline \multicolumn{2}{|r|}{ Clonidina } \\
\hline $\begin{array}{l}\text { Calidad } \\
\text { BAJA }\end{array}$ & $\begin{array}{l}\text { La clonidina ha mostrado un efecto discreto en la disminución de la } \\
\text { frecuencia de los sofocos }{ }^{165,178,181} \text {. } \\
\text { La clonidina puede presentar efectos adversos como sequedad de boca, } \\
\text { somnolencia, fatiga o náuseas y se desconoce sus efectos a largo plazo }{ }^{178} \text {. }\end{array}$ \\
\hline \multicolumn{2}{|r|}{ Gabapentina } \\
\hline $\begin{array}{c}\text { Calidad } \\
\text { MODERADA }\end{array}$ & $\begin{array}{l}\text { La gabapentina a dosis de } 900-2.400 \mathrm{mg} / \text { día se ha mostrado } \\
\text { moderadamente efectiva para la reducción de la frecuencia y la } \\
\text { intensidad de los sofocos frente a placebo }{ }^{173,192-194} \text {. } \\
\text { No obstante, la gabapentina puede presentar efectos adversos como } \\
\text { vértigo, somnolencia e inestabilidad }{ }^{190} \text {. }\end{array}$ \\
\hline $\begin{array}{c}\text { Calidad } \\
\text { MUY BAJA }\end{array}$ & $\begin{array}{l}\text { Frente a terapia estrogénica transdérmica, la gabapentina a bajas } \\
\text { dosis }(600 \mathrm{mg} / \mathrm{d}) \text { no ha mostrado diferencias significativas en cuanto } \\
\text { a la reducción de la frecuencia y la intensidad de los sofocos }{ }^{184} \text {. }\end{array}$ \\
\hline
\end{tabular}

\section{Recomendaciones}

\begin{tabular}{|c|l|}
\hline DÉBIL & $\begin{array}{l}\text { En mujeres durante la etapa de la peri y postmenopausia con } \\
\text { síntomas vasomotores moderados o intensos que empeoren su calidad } \\
\text { de vida y en las que esté contraindicado el tratamiento hormonal o } \\
\text { que no deseen tomarlo, y en las que las modificaciones del estilo de } \\
\text { vida resultan ineficaces, se sugiere valorar el tratamiento con } \\
\text { antidepresivos (paroxetina o desvenlafaxina) o gabapentina*. }\end{array}$ \\
\hline DÉBIL & $\begin{array}{l}\text { En las mujeres durante la peri o postmenopausia con síntomas } \\
\text { vasomotores moderados o intensos que afecten a su calidad de vida, } \\
\text { en las que el tratamiento hormonal, los antidepresivos y la } \\
\text { gabapentina están contraindicados o no deseen tomarlos, se puede } \\
\text { valorar la administración de clonidina. }\end{array}$ \\
\hline
\end{tabular}

* En el momento de la publicación de esta guía, la paroxetina, desvenlafaxina y gabapentina no tienen autorizada en España esta indicación. Según el Real Decreto 1015/2009 que regula la disponibilidad de medicamentos en situaciones especiales, los medicamentos utilizados fuera de sus indicaciones aprobadas requieren informar al paciente y/o a su familia, solicitar el consentimiento y reflejarlo en la historia clínica. 


\subsubsection{Fitoterapia}

\subsubsection{Fitoestrógenos}

Los fitoestrógenos son un grupo heterogéneo de sustancias químicas de origen vegetal que contienen componentes no esteroideos y que han mostrado actividad estrogénica leve en unos tejidos y antagonista o neutra en otros ${ }^{195}$.

Diversas RS han evaluado la eficacia de los fitoestrógenos en el tratamiento de los síntomas vasomotores ${ }^{120,196-200}$. Una de las RS incluyó 43 ECA con una duración mínima de 12 semanas y evaluó la eficacia y la seguridad de las intervenciones (farmacológicas o dietéticas) con alto contenido de fitoestrógenos ( $\geq 30 \mathrm{mg} / \mathrm{d}$ de isoflavonas) frente a la ausencia de tratamiento, placebo o terapia hormonal ${ }^{120}$. Los ECA incluyeron 4.364 mujeres en periodo peri y postmenopáusico con síntomas vasomotores (sofocos y sudores nocturnos), excluyendo aquellas con cáncer de mama o historia de cáncer de mama. Las intervenciones se clasificaron en: suplementos dietéticos de soja, extractos estandarizados de isoflavonas de soja, isoflavonas de trébol rojo, concentrados de genisteína y otros fitoestrógenos.

Además, se identificaron 9 ECAs, no incluidos en las RS anteriores, que evaluaron la eficacia de los fitoestrógenos para aliviar los síntomas asociados a la menopausia: uno comparando isoflavonas de trébol rojo frente a placebo ${ }^{201}$; cinco de isoflavonas de soja, tres frente a placebo ${ }^{202-204} \mathrm{y}$ dos frente a otros tratamientos ${ }^{200,205} ; \mathrm{y}$ tres de linaza (flaxseed) frente a placebo ${ }^{206-208}$.

\subsection{Isoflavonas de trébol rojo (Trifolium pratense; red clover)}

Se identificaron $1 \mathrm{RS}^{120}$ y 2 ECAs, uno de ellos ${ }^{209}$ incluido en la RS. El otro ECA, a doble-ciego ${ }^{201}$, evaluó la eficacia de las isoflavonas de trébol rojo (en extracto cuyo contenido de isoflavonas de trébol rojo equivale a una ingestión diaria de $160 \mathrm{mg}$ ) para aliviar los síntomas vasomotores y otros síntomas asociados a la menopausia frente a placebo en 113 mujeres tras un tratamiento de 12 semanas. Las pacientes fueron divididas en dos grupos de tratamiento -con isoflavonas o con placebo- durante 3 meses y posteriormente, tras una fase de lavado de una semana, fueron tratadas con el tratamiento contrario durante otros 3 meses. Las variables de medida fueron la frecuencia diaria de sofocos y sudores nocturnos y la escala combinada de síntomas Kupperman (KI). Los resultados se presentaron como reducción media porcentual de cada variable, desde el inicio hasta el final de cada tratamiento (3 meses).

En comparación a placebo, el tratamiento con un extracto Calidad estandarizado de isoflavonas de trébol rojo a dosis de 40-80 mg, mostró muy baja/ una reducción no significativa de 0,93 sofocos al día favorable al moderada tratamiento con isoflavonas (5 ECAs, 300 mujeres, DM -0,93, IC $95 \%$ $1,95$ a 0,10$)^{120}$. Un metanálisis de dos de los ECA anteriores (199 mujeres) $)^{179,181}$ mostró una reducción no significativa del $20 \%$ en el número diario de sofocos favorable al tratamiento, en comparación a placebo (DM 20,15; IC $95 \%-12,08$ a 52,38). 
No se han encontrado diferencias significativas entre el extracto de Calidad isoflavonas de trébol rojo (Promensil®) y el placebo en la proporción de muy baja/ mujeres que experimentaron algún efecto indeseado (RR 0,95; moderada IC $95 \% 0,65$ a 1,40), ni en la incidencia de infecciones del tracto respiratorio, cefalea, mialgias, náuseas, artralgias, diarrea o pérdidas vaginales (1 RS; 169 mujeres; 12 semanas), así como en el grosor endometrial (evaluado por ecografía) (1 RS; 51 mujeres; 12 semanas; DM 0,06; IC $95 \%-4,94$ a 5,06) ${ }^{120}$.

Las isoflavonas de trébol rojo (extracto), a dosis de $80 \mathrm{mg} / \mathrm{d}$, fueron Calidad significativamente más eficaces que placebo para reducir la frecuencia de moderada sofocos (DME -65,3\%; $\mathrm{p}<0,0001)$ y sudores nocturnos (DME -71,3\%; $\mathrm{p}<0,0001$ ) y mejorar la sintomatología de la menopausia (Escala combinada de síntomas (KI): DME $-68,7 \%$; $<<0,0001)$ tras 3 meses de tratamiento. Tras la segunda fase del tratamiento se encontró una reducción de la frecuencia diaria de sofocos (isoflavonas de trébol rojo $3,3 \pm 4$; placebo $9,3 \pm 5,2$ ) y de sudores nocturnos (isoflavonas de trébol rojo $1,7 \pm 1,8$; placebo $4,3 \pm 2,6)$ significativamente superiores $(\mathrm{p}<0,0001)$ a las observadas en el grupo placebo y en la mejora de la sintomatología de la menopausia (Escala combinada de síntomas (KI): isoflavonas de trébol rojo $9,7 \pm 9,4 v s$. placebo $26,5 \pm 14,9)(1 \mathrm{ECA}, 12 \text { semanas, } 113 \text { mujeres })^{201}$.

\subsection{Isoflavonas de soja: extractos, suplementos dietéticos, concentrados (genisteína)}

Trece ECA analizados en la RS ${ }^{120}$ evaluaron la eficacia de suplementos dietéticos de soja con alto contenido de isoflavonas ( $40-120 \mathrm{mg} /$ día), en forma de bebidas, harinas, alimentos con semillas de soja, cereales, productos lácteos, etc; frente a placebo u otros comparadores. De los trece ECA incluidos en esta $\mathrm{RS}^{120}$, doce (1.305 mujeres) evaluaron la eficacia de los extractos estandarizados de soja (isoflavonas $33-120 \mathrm{mg} / \mathrm{d}$ áa) frente a placebo $^{204,205,210-218}$ y frente a terapia hormonal ${ }^{219}$ y cuatro evaluaron la eficacia de los concentrados de genisteína $(30-60 \mathrm{mg} / \mathrm{d})$ frente a placebo o terapia hormonal, en el tratamiento de los sofocos en mujeres durante la etapa de la postmenopausia ${ }^{208,220,221,222}$. Adicionalmente, se han identificado tres revisiones sistemáticas más recientes, dos de ellas con metanálisis ${ }^{198,199}$, que evaluaron la eficacia de las isoflavonas de soja para aliviar los síntomas vasomotores asociados al climaterio (sofocos) frente a placebo, y la tercera, una RS de comparación indirecta ajustada de dos metanálisis de isoflavonas de soja y de THS (ambos frente a placebo) ${ }^{200}$. Una de estas RS/metanálisis ${ }^{198}$ evaluó 19 ECA, de más de 12 semanas de duración (máximo 96 semanas). Se incluyeron un total de 1.415 pacientes, excluyendo las pacientes con cáncer. Los ECA evaluaron la eficacia de las isoflavonas de soja en forma de suplementos dietéticos, extractos o concentrados (genisteína o daidzeína) frente a placebo para reducir la incidencia de sofocos asociados al climaterio. Los ECA incluidos expresaron sus resultados como: número de sofocos, escala de valoración de síntomas vasomotores o reducción porcentual de los sofocos, por unidad de tiempo (día, semana o mes). Otra de estas RS/metanálisis ${ }^{199}$, incluyó 19 ECA de duración superior a 6 semanas, en los que se evaluó la eficacia de los suplementos de isoflavonas de soja (extractos naturales o sintéticos) para aliviar los sofocos en mujeres en la etapa de la peri y postmenopausia, 
frente a placebo. En ellos se incluían pacientes con cáncer de mama. Se excluyeron los ECA de suplementos dietéticos y proteínas de soja, así como de otros productos que contenían isoflavonas, pero no de soja. También se excluyeron ECA con tratamientos adicionales que pudiesen influir en los sofocos. Las variables de eficacia consideradas fueron la frecuencia (diaria o semanal) y/o la gravedad (intensidad) de los sofocos. Los resultados del metanálisis en relación a la eficacia de isoflavonas de soja se presentaron como la diferencia media porcentual entre 6 semanas y 12 meses, en cuanto a la frecuencia (1.196 pacientes) y la gravedad de los sofocos (988 pacientes). De forma complementaria se realizó un análisis de la frecuencia de los sofocos por subgrupos, en función de la dosis de isoflavonas y genisteína, duración de la intervención y frecuencia y gravedad basal de los sofocos. No se dispone de información sobre la posible influencia del contenido de isoflavonas, la duración de la intervención, la gravedad basal de los sofocos u otros factores en los resultados relacionados con la gravedad de los mismos. Los ECA incluidos presentaron cierta heterogeneidad relacionada principalmente con la dosis de isoflavonas, duración, frecuencia y gravedad basal de los sofocos y escalas de medida de la gravedad o intensidad de los mismos, lo que podría limitar sus conclusiones. El contenido de isoflavonas de soja de los suplementos equivale a una ingestión diaria de 30-80 mg (media $54 \mathrm{mg} / \mathrm{d}$ ). Una RS con metanálisis ${ }^{200}$, evaluó la eficacia comparada de suplementos de isoflavonas de soja (extractos estandarizados o concentrados) y THS para aliviar la gravedad de los sofocos en mujeres en la etapa de la postmenopausia, mediante un metanálisis de comparaciones indirectas ajustadas, realizado a partir de un metanálisis de isoflavonas de soja, frente a placebo (10 ECA, 503 pacientes), y de otro metanálisis de TSH frente a placebo (9 ECA, 256 pacientes). El total de pacientes incluidos en el metanálisis final fue de 759 y la duración de los ECA era de 12-56 semanas. Los resultados presentados combinaron la frecuencia y la gravedad de los sofocos.

Se han identificado además 5 ECAs, no incluidos en las RS anteriores, que evaluaron la eficacia de las isoflavonas de soja para aliviar los síntomas asociados a la menopausia, tres frente a placebo ${ }^{202-204} \mathrm{y}$ dos frente a otros tratamientos ${ }^{205,223}$. Un ECA simple-ciego ${ }^{202}$, evaluó la eficacia de las isoflavonas de soja (en extracto, cuyo contenido equivale a una ingestión diaria de $60 \mathrm{mg}$ ) combinadas con berberina y otros componentes (calcio, vitaminas, etc) para mejorar la sintomatología asociada a la menopausia (variable secundaria), frente a placebo, en 120 mujeres con dislipemia. Los resultados se presentaron como reducción media porcentual de la intensidad de los sofocos, sudores nocturnos y otros síntomas, desde el inicio hasta el final del tratamiento (12 semanas). Dos ECA dobleciego $^{203,205}$, evaluaron la eficacia de un suplemento natural de isoflavonas de soja (S-equol) para reducir la frecuencia y la intensidad de los sofocos y de otros síntomas asociados a la menopausia. Uno de ellos ${ }^{203}$, evaluó la eficacia del S-equol (un metabolito de la diadzeina) (experimental) frente a placebo en 160 mujeres japonesas durante la etapa de la postmenopausia (mujeres que experimentaban al menos un sofoco al día). La sintomatología de la menopausia se valoró con las escalas combinadas de síntomas: CSE (Climacteric Symptom Evaluation Form Checklist), VAS (Visual Analog Scale) y subescalas de la GCS (Greene Climateric Scale). Adicionalmente, se valoró la calidad de vida con las escalas SF-36 y VAS. Los resultados se presentaron como reducción media de la frecuencia y de la intensidad de los sofocos, desde el inicio hasta el final del tratamiento a las 12 semanas. El otro, un ECA piloto ${ }^{205}$, evaluó la eficacia del S-equol a dosis de 10, 20 y 
$40 \mathrm{mg} / \mathrm{d}$, para reducir la frecuencia de los sofocos (variable primaria) y mejorar otros síntomas asociados a la menopausia (variables secundarias), frente a isoflavonas de soja (extracto), en 102 mujeres coreanas en la etapa de la postmenopausia. Los resultados se presentaron como reducción media de la frecuencia diaria de sofocos, desde el inicio hasta el final del tratamiento (8 semanas), para cada una de las dosis de S-equol, pero no de forma global. Un ECA simple-ciego ${ }^{204}$, evaluó la eficacia de las isoflavonas de soja (germen), a dosis de 84 y $126 \mathrm{mg}$ diarios, para reducir la frecuencia de los sofocos y mejorar la sintomatología asociada a la menopausia, frente a placebo, en 90 mujeres chinas durante la etapa de la postmenopausia. Las variables de eficacia fueron la frecuencia semanal de sofocos, puntuación en la escala Kupperman (KI) en las semanas 12 y 24, así como la reducción porcentual media de cada variable desde el inicio hasta el final del tratamiento a las 24 semanas, para ambas dosis. Los resultados se presentan de forma gráfica para cada una de las dosis de isoflavonas, pero no de forma global; mientras que los datos numéricos se especifican sólo en el resumen. Un $\mathrm{ECA}^{223}$ evaluó la eficacia de isoflavonas de soja combinadas con Lactobacillus sporogenes para reducir la intensidad de los sofocos y la sudoración nocturna, frente a la misma combinación con magnolia, en 634 mujeres durante la etapa de la postmenopausia que presentaban alteraciones psicoafectivas y/o del sueño (no graves). Los resultados se expresaron como reducción media porcentual de la intensidad de ambos síntomas, desde el inicio hasta el final del tratamiento a las 12 semanas.

Los resultados globales del metanálisis han mostrado una diferencia Calidad estandarizada media a favor de las isoflavonas de soja (en forma de muy baja suplementos dietéticos, extractos o concentrados) frente a placebo, en mujeres en la etapa peri o postmenopáusica: DME -0,391; IC $95 \%-0,529$ a 0,254; I2 =53,5\% (1 RS / metanálisis, 19 EC, duración > 12 semanas, 1.415 mujeres $)^{198}$. El análisis por subgrupos según el tipo de isoflavona considerado, también ha mostrado diferencias significativas frente a placebo:

- DME -0,20; IC 95 \% -0,46 a -0,06 (suplementos dietéticos) (10 ECAs)

- DME -0,51; IC $95 \%-0,79$ a -0,22 (extractos) (6 ECAs)

- DME -0,45; IC 95 \% -0,64 a -0,25 (concentrados) (3 ECAs).

Los suplementos proteicos de soja redujeron la intensidad de los Calidad sofocos (escala Kupperman) en una proporción significativamente mayor muy bajade mujeres $(84 \%)$ que el placebo $(60 \%)$, si bien, no se observaron moderada diferencias significativas entre ambos grupos en la frecuencia diaria de sofocos al final del tratamiento (RS; 1 ECA) $)^{120}$. Cuatro de los ECA incluidos en la $\mathrm{RS}^{120}$ y un ECA posterior ${ }^{204}$, mostraron una disminución de la frecuencia de sofocos (uno también de los sudores nocturnos) respecto a placebo. Dos ECAs incluidos en la $\mathrm{RS}^{120}$ y un ECA posterior ${ }^{205}$ mostraron una disminución de la intensidad de los sofocos medida con la escala de Kupperman y uno con una escala subjetiva de 1 a $3^{215}$. En otro $\mathrm{ECA}^{217}$, los sofocos y sudores nocturnos fueron significativamente más frecuentes en mujeres que habían sido previamente tratadas con isoflavonas de soja (48,4\%) que con placebo (31,7\%); aunque ésta fue una variable secundaria del estudio. Los otros ECA incluidos en la $\mathrm{RS}^{120}$ no mostraron diferencias significativas en variables de eficacia, 
en comparación con placebo ${ }^{211,214}$ y con terapia hormonal ${ }^{219}$. En mujeres tratadas con un suplemento proteico de soja frente a mujeres tratadas con placebo, la reducción fue del 57 \% (escala Kupperman) (RS; 1 ECA) ${ }^{224}$; en mujeres tratadas con un producto lácteo rico en soja (asociado o no al ejercicio físico) frente a un grupo control no especificado la reducción fue del $72 \%$ (RS; $1 \mathrm{ECA})^{207}$. Los suplementos proteicos de soja redujeron significativamente la frecuencia diaria de sofocos frente a placebo $\left(45 \%\right.$ vs. 30 \%) $(-1,59$; IC $95 \%-1,95$ a $-1,2 ; \mathrm{p}<0,01)(\mathrm{RS} ; 1 \text { ECA })^{225}$. Los suplementos dietéticos de soja mostraron resultados favorables, reduciendo la frecuencia $\mathrm{y} / \mathrm{o}$ intensidad de los sofocos de forma significativa (RS; 6 ECAs) ${ }^{207,225-229}$. Los siete ECA restantes no mostraron diferencias significativas frente al grupo control ${ }^{230-236}$.

Los suplementos dietéticos de soja mostraron diferencias significativas favorables para mejorar los sofocos en mujeres en la etapa de la postmenopausia, aunque no se clarifica si se trata de la frecuencia y/o la intensidad de los mismos (RS; 2 ECAs).

En mujeres en la etapa de la postmenopausia, la dieta enriquecida en soja redujo de forma significativa la intensidad de los sofocos frente a una dieta normal $(\mathrm{p}=0,004)$, mediante un cuestionario realizado a las mismas $(\mathrm{RS} ; 1 \mathrm{ECA})^{226}$.

En mujeres tratadas con suplementos proteicos de soja $(-50 \%)$ y con terapia hormonal (estradiol + noretisterona) $(-46 \%)$, frente a placebo (-29 \%) se encontró una mejoría significativa de los síntomas vasomotores asociados a la menopausia (síntomas "somáticos" en la Menopause Rating Scale) (RS; 1 ECA) $)^{228}$.

El tratamiento con un concentrado de genisteína a dosis de $54 \mathrm{mg} /$ día Calidad se asoció a una disminución significativa del número (56,4 \%) e intensidad muy baja$(37,5 \%)$ de los sofocos en comparación con placebo a los 12 y 24 meses (1 moderada ECA; 389 mujeres aleatorizadas en un estudio sobre metabolismo óseo; subanálisis de 236 mujeres con sofocos) $)^{222}$. Los concentrados de genisteína a dosis de 30 y $60 \mathrm{mg} / \mathrm{d}$ redujeron significativamente la frecuencia diaria de sofocos $(51,2 \%$ y $41,2 \%)$ en comparación con placebo $(27,2 \%$ y $29,3 \%$ ) respectivamente, si bien, no hubo diferencias significativas en cuanto a la intensidad de los sofocos, medida con Greene Climateric Scale o valorada por el investigador (RS; 2 ECAs; 264 mujeres; 12 semanas) ${ }^{120}$.

En mujeres tratadas con un concentrado de genisteína a dosis de $54 \mathrm{mg} /$ día y con tratamiento hormonal en comparación a placebo, se observó una disminución del $24 \%$ en los sofocos diarios frente al placebo y una reducción del $30 \%$ con el tratamiento hormonal en comparación a la genisteína $(\mathrm{p}<0,05)(1 \mathrm{ECA} ; 90 \text { mujeres })^{221}$.

Los extractos de isoflavonas de soja (naturales o sintéticos) se Calidad mostraron significativamente más eficaces que placebo para reducir la moderada frecuencia de sofocos en mujeres en la etapa de la peri y postmenopausia: 
DM -20,62 \%; IC $95 \%$-29,38 a -12,86; p < 0,00001; I2 = 67 \% (1 revisión sistemática / metanálisis, 13 ECA, 6 semanas-12 meses, 1.196 mujeres). La reducción de la frecuencia de los sofocos fue significativamente mayor con los suplementos de soja de mayor contenido de isoflavonas y/o con intervenciones de duración superior a 12 semanas; e independiente de la frecuencia basal de sofocos ${ }^{199}$. Los resultados del metanálisis mostraron que los extractos de isoflavonas de soja (naturales o sintéticos) redujeron significativamente frente a placebo la gravedad (intensidad) de los sofocos en mujeres en la etapa de la peri y postmenopausia: DM -26,2\%; IC $95 \%-42,23$ a -10,15; $\mathrm{p}=0,001 ; \mathrm{I} 2$ = $86 \%$ (1 RS / metanálisis, 10 EC, 12 semanas-12 meses, 988 mujeres) ${ }^{199}$.

La terapia hormonal se mostró más eficaz que los extractos de Calidad isoflavonas de soja (estandarizados o concentrados) para aliviar los sofocos muy baja (frecuencia y gravedad) en mujeres en la etapa de la postmenopausia: DME 0,84; IC $95 \%-1,33$ a $-0,35 ;$ I $2=84,7 \%$ (1 metanálisis de comparaciones indirectas ajustadas, $19 \mathrm{EC}, 12-56$ semanas, 759 mujeres $)^{200}$.

Las isoflavonas de soja (extracto) combinadas con berberina se Calidad mostraron significativamente superiores a placebo para reducir la muybaja intensidad de los sofocos (DME $-23,8 \%$; $<0,001$ ) y la sudoración nocturna (DME -50 \%; $<<0,01$ ) en mujeres en climaterio con dislipemia, tras 12 semanas de tratamiento (1 ECA, 120 mujeres, 12 semanas $)^{202}$.

El S-(-) equol (suplemento de isoflavonas de soja) se mostró Calidad significativamente superior al placebo para reducir la frecuencia diaria de muy baja sofocos (DME -0,9 (-24,2\%); $\mathrm{p}=0,009)$ y la intensidad de los mismos (escala CSE) (DME -0,2 (-16,2 \%); $\mathrm{p}=0,012)$ en mujeres japonesas en la etapa de la postmenopausia, tras 12 semanas de tratamiento (1 ECA, 160 mujeres, 12 semanas ${ }^{203}$. El contenido de isoflavonas de soja (suplemento) equivale a una ingestión diaria de $10 \mathrm{mg}$.

El S-equol (suplemento de isoflavonas de soja) no mostró diferencias Calidad significativas frente a isoflavonas de soja (extracto) para reducir la frecuencia muy baja diaria de sofocos en mujeres coreanas durante la postmenopausia, tras 8 semanas de tratamiento, independientemente de la dosis [DME -0,55; $\mathrm{p}=0,528$ (S-equol $10 \mathrm{mg} / \mathrm{d}$ ); DME -0,74; $\mathrm{p}=0,380$ (S-equol $20 \mathrm{mg} / \mathrm{d}$ ); DME 0,17; $\mathrm{p}=0,841$ (S-equol $40 \mathrm{mg} / \mathrm{d}$ )] (1 ECA, 102 mujeres, 8 semanas ${ }^{205}$.

Otro ECA mostró que las isoflavonas de soja (germen) fueron Calidad significativamente superiores a placebo para reducir la frecuencia semanal muy baja de sofocos y mejorar la sintomatología de la menopausia en mujeres chinas en la etapa de la postmenopausia, tras 24 semanas de tratamiento (1 ECA, 90 mujeres, 24 semanas) ${ }^{204}$.

- Frecuencia semanal de sofocos ( $\mathrm{p}<0,01)$ : DME: -16,5 (84 mg/d); DME: -20,7 (126 mg/d)

- Escala combinada de síntomas (KI) (p < 0,01): DME: -23,2 (84 mg/d); DME: -22,1 (126 mg/d). 
La combinación de isoflavonas de soja con Lactobacillus sporogenes Calidad no mostró diferencias significativas frente a la misma combinación con muy baja magnolia, para reducir la intensidad de los sofocos (DM 0,5\%, no significativo) y la sudoración nocturna (DM 7,6\%, no significativo) en mujeres en la postmenopausia, tras 12 semanas de tratamiento (1 ECA, 634 mujeres, 12 semanas ${ }^{223}$. El contenido de isoflavonas de soja equivale a una ingestión diaria de $60 \mathrm{mg}$.

\subsection{Otros fitoestrógenos}

Cinco ECA analizados en la RS ${ }^{120}$ evaluaron la eficacia de otros fitoestrógenos frente a placebo u otros comparadores, en el tratamiento de los síntomas vasomotores. Dos de estos ECA compararon suplementos dietéticos de linaza y de soja con placebo ${ }^{232,235}$ y uno comparó extractos y suplementos dietéticos de linaza frente a placebo ${ }^{206}$. Otro ECA comparó frente a placebo un tipo de extracto de lúpulo ${ }^{237}$ y el último ${ }^{238}$, el extracto $\mathrm{ERr}$ 731 de ruibarbo (Rheum rhaponticum) frente a placebo.

Además se identificaron tres ECAs posteriores a esta RS. Un ECA doble-ciego ${ }^{208}$, con 38 pacientes, evaluó la eficacia de un suplemento dietético de linaza (rebanadas de pan) para reducir la frecuencia de los sofocos y los sudores nocturnos y mejorar la sintomatología asociada a la menopausia, frente a placebo. De forma complementaria, se evaluó el efecto sobre el grosor del endometrio. Los resultados se presentaron como frecuencia diaria media de sofocos y sudores nocturnos y puntuación en la escala KI al final del tratamiento en la semana 12. No se especifica el valor de $\mathrm{p}$ ni el IC de los resultados. Otro $\mathrm{ECA}^{206}$, evaluó la eficacia del extracto de linaza (cápsulas) y de un suplemento dietético de linaza (polvo) para mejorar los sofocos y otros síntomas vasomotores (variables secundarias) y los síntomas vaginales asociados a la menopausia (variable primaria), frente a placebo y entre ambos preparados de linaza, en 90 mujeres brasileñas en la etapa peri y postmenopausia. Los resultados se presentaron como reducción media de la intensidad de los sofocos y de la puntuación en la escala KI, desde el inicio hasta el final del tratamiento a los 6 meses. No se especificó el IC de todos los resultados, ni el valor de $\mathrm{p}$ de las diferencias frente a placebo, ni entre ambos tratamientos. Un ECA doble-ciego ${ }^{207}$, evaluó la eficacia de un suplemento de linaza (barritas) para reducir los sofocos (frecuencia e intensidad), frente a placebo, en 188 mujeres durante la postmenopausia. De forma complementaria (variables secundarias), se evaluaron los efectos adversos y el efecto sobre otros síntomas asociados a la menopausia y sobre la calidad de vida, con diferentes escalas de medida (Hot Flash Related Daily Interference Scale, Profile of Mood States, Menopause Quality of Life Scale, Global Impression of Benefit y cuestionario de efectos adversos). Los resultados se presentaron como reducción media porcentual de la frecuencia de sofocos y de la puntuación en la escala combinada de sofocos, desde el inicio hasta el final del tratamiento a las 6 semanas. Sólo se especifica el IC de algunos resultados.

Los extractos o suplementos dietéticos de linaza cuyo contenido Calidad baja equivale a una ingestión diaria de $25 \mathrm{~g}$ ( $46 \mathrm{mg}$ de lignanos) no mostraron o muy baja diferencias significativas frente a placebo, en la frecuencia o la intensidad de los sofocos (3 ECAs) $)^{206}$. 
La linaza en forma de suplemento dietético no mostró diferencias Calidad significativas frente a placebo para reducir la frecuencia diaria de sofocos muy baja y sudores nocturnos (DME 1,2; no significativo), ni para mejorar la sintomatología de la menopausia (Escala combinada de síntomas (KI): DME -0,3; no significativo) en mujeres en la etapa de la postmenopausia, tras 12 semanas de tratamiento (1 ECA, 12 semamas, 38 mujeres) ${ }^{208}$.

El suplemento dietético de linaza cuyo contenido equivale a una Calidad ingestión diaria de $90 \mathrm{mg}$ ( $270 \mathrm{mg}$ de lignano estandarizado o SDG, no muy baja mostró diferencias significativas frente a placebo para reducir la intensidad de los sofocos (DME - 0,83 ; no significativo) y de otros síntomas vasomotores asociados a la menopausia (Escala combinada de síntomas (KI): DME -2,22; no significativo) en mujeres brasileñas en la etapa de la postmenopausia, tras 6 meses de tratamiento (1 ECA, 6 meses, 90 mujeres) $)^{206}$.

La linaza en forma de suplemento dietético no mostró diferencias Calidad significativas frente a placebo para reducir la frecuencia semanal de muy baja sofocos $(\mathrm{DM}-1 \% ; \mathrm{p}=0,9)$ y la puntuación en la escala combinada de sofocos (frecuencia e intensidad) (DM -1,4; IC $95 \%-0,7$ a $-3,5 ; \mathrm{p}=0,29)$ en mujeres en la postmenopausia, tras 6 semanas de tratamiento (1 ECA, 6 semanas, 188 mujeres) $)^{207}$.

El contenido de linaza (suplemento dietético) equivale a una ingestión diaria de 7,5 g (410 mg de lignanos).

El extracto de linaza no mostró diferencias significativas frente a Calidad placebo para reducir la intensidad de los sofocos (DME -1,38; no muy baja significativo) y de otros síntomas vasomotores asociados a la menopausia (Escala combinada de síntomas (KI): DME -1,22; no significativo) en mujeres brasileñas en la etapa de la postmenopausia, tras 6 meses de tratamiento (1 ECA, 6 meses, 90 mujeres $)^{206}$. El contenido de extracto de linaza equivale a una ingestión diaria de $1 \mathrm{~g}$ (100 $\mathrm{mg}$ de lignano estandarizado o SDG).

No se observaron diferencias significativas entre los extractos o Calidad baja suplementos dietéticos de linaza y los suplementos dietéticos de soja o muy baja frente a placebo (3 ECAs) ${ }^{232,235}$.

Los extractos de lúpulo no mostraron diferencias significativas frente Calidad baja a placebo en la intensidad de los sofocos (escala Kupperman) ${ }^{237}$.

o muy baja

El extracto ERr 731 de ruibarbo (Rheum rhaponticum) redujo Calidad baja significativamente la intensidad (Menopause Rating Scale) y la frecuencia o muy baja de los sofocos y sudores nocturnos (reducción media de la puntuación: 2,5 vs. 1,2) comparado con placebo (RS; 1 ECA) ${ }^{238}$.

No se observaron diferencias significativas entre los dos preparados de Calidad linaza (extracto y suplemento dietético) para reducir la intensidad de los muy baja sofocos (DME -0,55; no significativo) y de otros síntomas vasomotores asociados a la menopausia (Escala combinada de síntomas (KI): DME -1,0; 
no significativo) en mujeres brasileñas en la etapa de la postmenopausia, tras 6 meses de tratamiento (1 ECA, 6 meses, 90 mujeres) ${ }^{206}$.

El contenido de linaza (extracto) utilizado equivale a una ingestión diaria de $1 \mathrm{~g}$ (100 mg de lignano estandarizado o SDG) y el de linaza (suplementos), a una ingestión diaria de $90 \mathrm{mg}$ ( $270 \mathrm{mg}$ de lignano estandarizado o SDG).

\subsection{Seguridad de los fitoestrógenos}

Se identificaron dos RS $(212,213)$ que evaluaron la seguridad de los fitoestrógenos. Una RS evaluó la seguridad de los tratamientos con fitoestrógenos (definidos como productos que contienen cierta cantidad de isoflavonas, lignanos o cumestanos) en mujeres de cualquier edad y que recibían la intervención por cualquier condición. La calidad global de los resultados fue muy baja debido principalmente a la combinación de poblaciones e intervenciones diversas y a limitaciones en el diseño. Se incluyeron 92 estudios con un total de 9.629 participantes ${ }^{239}$. Otra RS evaluó la relación entre el consumo de lignanos (procedentes del consumo en la dieta de productos como las leguminosas, te o café) y el riesgo de cáncer de mama ${ }^{240}$. Esta revisión incluyó un total de 12 estudios observacionales (cohortes y casos y controles). La revisión proporcionó un estimador conjunto de ambos tipos de diseño para el riesgo de cáncer de mama según un consumo reducido o elevado de lignanos. Los puntos de corte considerados variaron de forma importante entre los estudios (de 0,4 microg a 2,3 mg/día para consumo reducido y de 3,4 microg a $653 \mathrm{mg}$ /día para consumo elevado). Ninguno de los estudios ajustó el riesgo de cáncer de mama en función del tratamiento hormonal. No se puede descartar que las mujeres en la etapa de la postmenopausia que realizaron una dieta rica en lignanos (procedentes entre otros de fitoestrógenos) sean precisamente aquellas que no realizaron tratamiento hormonal.

La incidencia global de efectos indeseados fue similar entre los Calidad grupos de intervención (36,7\%) y control (38\%), sin diferencias muy baja significativas entre ellos (RR 1,01; IC $95 \%$ 0,95 a 1,08). Sólo los efectos gastrointestinales fueron más frecuentes entre el grupo de intervención $\left(17,9 \%\right.$ frente a 13,4 \%) (RR 1,28; IC $95 \%$ 1,08 a 1,50; 592 eventos) ${ }^{239}$.

Se observó una reducción no significativa del riesgo de cáncer de Calidad mama en el grupo con un elevado consumo de lignanos, con elevada muy baja variabilidad entre los diferentes estudios. La reducción de riesgo fue significativa en el subgrupo de mujeres en la etapa de la postmenopausia (OR 0,85 ; IC $95 \% 0,78$ a 0,93$)^{240}$.

\subsubsection{Cimifuga Racemosa (Black cohosh)}

Se identificaron tres $\mathrm{RS}^{241-243}$ y un $\mathrm{ECA}^{244}$ posterior que analizaron los resultados de la cimifuga racemosa para el alivio de los síntomas asociados a la menopausia. Dos RS analizaron la eficacia de Cimifuga Racemosa (Black cohosh) para aliviar los síntomas asociados a la menopausia ${ }^{241,242}$ y otra analizó sus efectos sobre la función hepática y posible hepatotoxicidad ${ }^{243}$. Dos de ellas realizan metanálisis de los resultados ${ }^{241,243}$. Una RS / metanálisis ${ }^{241}$ analizó 16 ECA (duración media 23 semanas, 2.027 pacientes), para 
evaluar la eficacia y/o seguridad de Cimifuga Racemosa en monoterapia (dosis media $40 \mathrm{mg} /$ día) en el tratamiento de los síntomas en mujeres en etapa de peri y postmenopausia. Se excluyeron los ECA de preparados combinados. Los ECA utilizaron como comparadores placebo, terapia hormonal, otros preparados de fitoterapia (trébol rojo) y fluoxetina. Las variables primarias fueron los síntomas vasomotores (frecuencia e intensidad de sofocos), síntomas vulvovaginales, escalas combinadas de síntomas de la menopausia y efectos adversos. Las escalas combinadas de síntomas fueron: KI (Kupperman Index), GCS (Greene Climateric Scale) y/o MRS (Menopause Rating Scale). Una RS ${ }^{242}$ analizó 12 ECA (1.573 mujeres), para evaluar la eficacia de Cimifuga Racemosa, Hypericum perforatum y Agnus castus, en monoterapia o combinados, en el tratamiento de los síntomas vasomotores, vaginales y psicosociales asociados a la menopausia. La duración de los ECA oscilaba entre 12 semanas y 12 meses; y la mayoría (11 ECA) eran frente a placebo y uno frente a terapia hormonal y tibolona. Los criterios de inclusión y exclusión de los ECA fueron amplios e imprecisos. La valoración de los EC se realizó con la escala Jadad (todos $>3$ ). Una RS/metanálisis ${ }^{243}$ de 5 ECA (algunos no publicados) evaluó el efecto sobre la función hepática y la posible hepatotoxicidad de Cimifuga Racemosa en un total de 1.117 mujeres en etapa peri y postmenopausica (1.020 finalizaron el estudio), excluyendo pacientes con cáncer. La duración de los ECA era de 3-6 meses y Cimifuga Racemosa se administró en forma de extracto isopropanólico (dosis equivalente a 40-180 mg/día de la planta), en monoterapia o asociación (con hierba de San Juan). Dos ECA compararon diferentes dosis de Cimifuga Racemosa, otros dos eran comparativos frente a placebo y uno frente a tibolona. Los parámetros de función hepática evaluados fueron las enzimas hepáticas: aspartatoaminotransferasa (AST), alaninoaminotransferasa (ALT) y gammaglutamiltranspeptidasa (g-GT). Los resultados se presentaron por subgrupos, para cada una de las enzimas hepáticas analizadas, pero no de forma global.

Se identificó además un ECA reciente, Ross $2012^{244}$, no incluido en las RS anteriores, que evaluó la eficacia de Cimifuga Racemosa para aliviar los síntomas asociados a la menopausia frente a placebo. Un ECA doble-ciego (12 semanas, 304 mujeres) ${ }^{244}$ no incluído en las RS anteriores, evaluó la eficacia y seguridad de Cimifuga Racemosa frente a placebo para mejorar la sintomatología asociada a la menopausia (MRS: Menopause Rating Scale) y su efecto específico sobre los sofocos (frecuencia y/o intensidad), atrofia y alteraciones psíquicas (subescalas de la MRS). De forma complementaria (variables secundarias) se evaluaron la seguridad y el efecto sobre algunas subescalas de la MRS (sofocos, atrofia, alteraciones psíquicas). Los resultados se presentaron como reducción media en la puntuación de la escala MRS y de la subescala de sofocos (frecuencia y/o intensidad), desde el inicio hasta el final del tratamiento a las 12 semanas.

Según los resultados del metanálisis ${ }^{243}$, Cimifuga Racemosa no Calidad modificó significativamente los parámetros de la función hepática muy baja (enzimas AST, ALT y g-GT) en ECA frente a placebo y tibolona, en mujeres en etapa peri y postmenopausica:

$$
\begin{aligned}
& \text { DME } 0,055 \pm 0,062(\mathrm{SEM}) ; \text { IC } 95 \%-0,066 \text { a } 0,176 ; \mathrm{p}=0,37(\mathrm{AST}) \\
& \text { DME } 0,063 \pm 0,062(\mathrm{SEM}) ; \text { IC } 95 \%-0,058 \text { a } 0,185 ; \mathrm{p}=0,31(\mathrm{ALT}) . \\
& \text { DME } 0,073 \pm 0,063(\mathrm{SEM}) ; \text { IC } 95 \%-0,050 \text { a } 0,196 ; \mathrm{p}=0,24(\mathrm{~g}-\mathrm{GT}) .
\end{aligned}
$$


La duración del tratamiento o la dosis administrada no influyeron Calidad en el efecto de Cimifuga Racemosa sobre la función hepática. La DME muy baja máxima encontrada $(0,1)$ fue inferior al límite considerado relevante $(0,2)$. Se concluyó que Cimifuga Racemosa producía un aumento de las enzimas hepáticas entre 5\%-10\%, que podría considerarse marginal y clínicamente no relevante.

Cimifuga Racemosa se mostró significativamente más eficaz que Calidad placebo para reducir la puntuación de la escala combinada de síntomas muy baja (MRS): $\mathrm{p}<0,001 ; \mathrm{y}$ de la subescala de sofocos: $\mathrm{p}=0,007$.

Cimifuga Racemosa no mostró diferencias significativas frente a Calidad placebo en la frecuencia diaria de sofocos en mujeres en la etapa de moderada la peri y postmenopausia (DM 0,07 ; IC $95 \%-0,43$ a 0,56 ; $\mathrm{p}=0,79$; I 2 = 47 \%) (1 revisión sistemática / metanálisis, 3 EC, 393 mujeres $)^{241}$.

Cimifuga Racemosa no mostró diferencias significativas frente a Calidad placebo en la intensidad de los sofocos en mujeres en la etapa de la peri y moderada postmenopausia (DM 0,12; IC $95 \%-0,06$ a 0,$30 ; \mathrm{p}=0,19 ; \mathrm{I} 2=42 \%$ ) (1 revisión sistemática / metanálisis, 3 EC, 214 mujeres) ${ }^{241}$.

Cimifuga Racemosa no mostró diferencias significativas frente a Calidad placebo en la sudoración nocturna (frecuencia) en mujeres en la etapa moderada de la peri y postmenopausia (DM 0,27; IC $95 \%-0,16$ a 0,$70 ; \mathrm{p}=0,21$ ) (1 revisión sistemática / metanálisis, 1 EC, 164 mujeres $)^{241}$.

Cimifuga Racemosa no mostró diferencias significativas frente a Calidad placebo para mejorar la sintomatología de la menopausia (escalas moderada combinadas de síntomas KI, GCS y/o MRS) en mujeres en la etapa de la peri y postmenopausia (DME -0,10; IC $95 \%-0,32$ a 0,$11 ; \mathrm{p}=0,34$; I $2=21 \%$ ) (1 revisión sistemática / metanálisis, 4 EC, 357 mujeres $)^{241}$.

Cimifuga Racemosa no mostró diferencias significativas frente a Calidad baja placebo en los efectos adversos en mujeres en la etapa de la peri y postmenopausia (RR 1,04; IC $95 \% 0,82$ a 1,32; p =0,74) (1 revisión sistemática / metanálisis, 2 EC, 344 mujeres) ${ }^{241}$.

Cimifuga Racemosa no modificó significativamente los parámetros Calidad de la función hepática (enzimas AST, ALT y g-GT), en EC frente a muy baja placebo y tibolona, en mujeres en la etapa de la peri y postmenopausia [DME 0,055 $\pm 0,062$ (SEM); IC $95 \%-0,066$ a 0,176; $\mathrm{p}=0,37$ (AST); DME $0,063 \pm 0,062$ (SEM); IC $95 \%-0,058$ a 0,$185 ; \mathrm{p}=0,31$ (ALT); DME $0,073 \pm 0,063 \quad(\mathrm{SEM}) ; \quad$ IC $95 \%-0,050$ a 0,$196 ; \mathrm{p}=0,24 \quad$ (g-GT)]. La duración del tratamiento o la dosis administrada no influyó en el efecto de Cimifuga Racemosa sobre la función hepática. La DME máxima encontrada $(0,1)$ es inferior al límite considerado relevante $(0,2)$. Se concluye que Cimifuga Racemosa produce un aumento de las enzimas hepáticas entre $5 \%-10 \%$, que puede considerarse marginal y clínicamente no relevante (1 metanálisis, 5 EC, duración 3-6 meses, 1.117 mujeres) ${ }^{243}$. 
Cimifuga Racemosa se mostró significativamente más eficaz que Calidad placebo para reducir los sofocos (frecuencia y/o intensidad) y otros muy baja síntomas asociados a la menopausia, tras 12 semanas de tratamiento (1 ECA, 12 semanas, 304 mujeres) $)^{220}$.

* Escala combinada de síntomas (MRS): $\mathrm{p}<0,001$.

* Escala de sofocos (subescala MRS): $\mathrm{p}=0,007$.

\subsubsection{Otros tratamientos de fitoterapia}

Se han identificado dos $\mathrm{RS}^{242,245}$ que analizan la eficacia de otros tratamientos de fitoterapia para mejorar la sintomatología asociada a la menopausia: Hypericum perforatum (entre otros tratamientos) ${ }^{242}$, y Lepidium meyenii ${ }^{245}$. Ninguna de las dos realizó metanálisis de los resultados.

Adicionalmente se han identificado cuatro ECA, no incluidos en las RS anteriores, que analizaron la eficacia de otros tratamientos de fitoterapia para aliviar los síntomas asociados a la menopausia, frente a placebo [Panax ginseng ${ }^{246}$, Pimpinella anisum $^{247}$, regaliz (licorice $)^{248} \mathrm{y}$ valeriana $\left.{ }^{249}\right]$.

\subsection{Hypericum perforatum o Hierba de San Juan (hipérico)}

Una $\mathrm{RS}^{242}$ analizó 12 ECA (1.573 mujeres), para evaluar la eficacia de Cimifuga Racemosa, Hypericum perforatum y Agnus castus, en monoterapia o combinados, en el tratamiento de los síntomas vasomotores, genitales y psicosociales asociados a la menopausia. Los resultados relativos a Hypericum perforatum provienen de un ECA (16 semanas, 100 mujeres) que evaluó su eficacia (asociado a Agnus castus) ${ }^{250}$.

Dos ECA (147 mujeres) que compararon hipérico con placebo Calidad baja mostraron una reducción en el número de sofocos $(-0,88$; IC $95 \%-1,24$ a -0,52) (223-224), pero la intensidad sólo disminuyó en uno de ellos ${ }^{251}$.

Un ECA doble ciego (301 mujeres) evaluó la eficacia de una Calidad baja combinación de Hierba de San Juan con Cimifuga Racemosa. A las 16 semanas, el grupo que recibió tratamiento mostró una reducción más marcada de una escala de síntomas de la menopausia (MRS: Menopause Rating Scale) en comparación con placebo, estadísticamente significativa ${ }^{252}$.

Se han descrito posibles interacciones de hipérico con varios Calidad fármacos metabolizados por un enzima hepático (citocromo P-450) como moderada carbamacepina, ciclosporina, anticonceptivos orales, nifedipina, etc., así como el riesgo de padecer un síndrome serotoninérgico al tomarse conjuntamente con antidepresivos ${ }^{253-256}$.

\subsection{Otras hierbas medicinales}

Una $\mathrm{RS}^{245}$ analizó 4 ECA (dos publicados en un solo artículo), para evaluar la eficacia de Lepidium meyenii (Maca) (2-3,5 g/día) en monoterapia o combinado, frente a placebo, en el tratamiento de los síntomas asociados a la menopausia, en un total de 
202 mujeres ${ }^{257-259}$. La duración de los ECA osciló entre 6 semanas y 3 meses. Las variables analizadas incluyeron síntomas psicológicos, somáticos, vasomotores y sexuales; y las escalas de medida utilizadas (síntomas combinados) fueron: GCS (Greene's Climateric Store) y KMI (Kupperman's Menopausal Index). En la misma RS, un ECA que comparó Dong quai (Angelica sinensis) (71 mujeres) frente a placebo y otro que comparó la hierba del asno (Evening Primrose: Oenothera biennis) (56 mujeres) frente a placebo no encontraron diferencias estadísticamente significativas entre tratamiento activo y placebo. Un ECA que comparó ginseng (Panax ginseng) (384 mujeres) frente a placebo no encontró diferencias en relación a los sofocos entre ambos tratamientos. Otros cuatro ECA más incluidos en la revisión de Huntley analizaron distintas combinaciones de hierbas, y en ninguno de ellos se pudieron concluir beneficios claros del tratamiento activo. Una RS evaluó el efecto de un combinado chino de seis hierbas medicinales, conocido como "decocción ER" y localizó cinco ECA realizados y publicados en China (557 mujeres) $)^{260}$.

Lepidium meyenii se mostró significativamente más eficaz que Calidad placebo para aliviar los síntomas asociados a la menopausia según las muy baja escalas combinadas de síntomas GCS: $\mathrm{p}<0,05^{257}, \mathrm{p}<0,001^{259}$; y la escala KMI: $\mathrm{p}<0,001^{259}, \mathrm{p}<0,05^{258}$ (1 revisión sistemática, $4 \mathrm{EC}$, duración 6 semanas-3 meses, 202 mujeres).

El tratamiento combinado chino de hierbas conocido como la Calidad "decocción ER" mostró mejoras estadísticamente significativas en los muy baja síntomas menopáusicos en comparación con tratamientos no hormonales, pero no comparando con tratamientos hormonales.

Se identificó un ECA doble-ciego (12 semanas) que evaluó la eficacia de Panax ginseng frente a placebo para mejorar los sofocos y otros síntomas asociados a la menopausia, en 72 mujeres coreanas en la etapa postmenopáusica ${ }^{246}$. Las escalas de medida fueron: Kuppermann index (KI) y Menopause Rating Scale (MRS). También se analizó el efecto sobre factores de riesgo cardiovascular. Los resultados se presentaron como reducción media de los sofocos y de la puntuación global de la sintomatología, para las dos escalas de medida, desde el inicio hasta el final del tratamiento a las 12 semanas.

Panax ginseng se mostró significativamente más eficaz que placebo Calidad para reducir la sintomatología asociada a la menopausia en mujeres muy baja coreanas en el periodo de la postmenopausia, tras 12 semanas de tratamiento (puntuación media global en las dos escalas) (1 ECA, 12 semanas, 72 mujeres $)^{246}$.

* Escala combinada de síntomas:

DME -5,5; $\mathrm{p}=0,032$ (escala KI).

DME -3,15; $\mathrm{p}=0,035$ (escala MRS).

$\mathrm{Su}$ eficacia para reducir los sofocos, presentó resultados variables frente a placebo en función de la escala de medida utilizada. 
* Escala de sofocos:

DME -1,24; $\mathrm{p}=0,046$ (escala KI).

DME - 0,$83 ; \mathrm{p}=0,120$ (escala MRS).

Un ECA doble-ciego (8 semanas), que evaluó la eficacia de Pimpinella anisum frente a placebo, para reducir los sofocos (frecuencia e intensidad), en 72 mujeres de Irán en etapa de postmenopausia ${ }^{247}$. Las variables evaluadas fueron la reducción media de la frecuencia y la intensidad de los sofocos desde el inicio hasta el final del tratamiento a las 8 semanas. El estudio incluyó pocos pacientes y fue de corta duración.

Pimpinella anisum resultó significativamente más eficaz que placebo Calidad para reducir la frecuencia $(\mathrm{DM}-2,62 ; \mathrm{p}<0,001)$ y la intensidad (DM - muy baja 33,34; $\mathrm{p}<0,001)$ de los sofocos en mujeres de Irán, tras 8 semanas de tratamiento (1 ECA, 8 semanas, 72 mujeres) $)^{247}$.

Un ECA doble-ciego (12 semanas), evaluó la eficacia del regaliz (Liquorice) frente a placebo para reducir los sofocos (frecuencia e intensidad) en 90 mujeres de Irán ${ }^{248}$. La intensidad de los sofocos se valoró con un cuestionario basado en una escala linear visual ("pain ruler"). Las variables evaluadas se presentaron como reducción media de la frecuencia de sofocos desde el inicio hasta las semanas 8 y 12 y la distribución porcentual de pacientes en función de la gravedad de los sofocos (leves, moderados o graves), antes del tratamiento y en las semanas 8 y 12 .

El regaliz ("Liquorice") resultó significativamente más eficaz que Calidad placebo para reducir la frecuencia diaria de sofocos, tras 8 semanas de muy baja tratamiento, pero no mostró diferencias significativas tras 12 semanas: DM -1,3; $\mathrm{p}<0,001$ (semana 8); DM -0,09; (no significativo) (semana 12) (1 ECA, 12 semanas, 90 mujeres $)^{248}$.

Se identificó un ECA doble-ciego ( 8 semanas) evaluó la eficacia de la valeriana frente a placebo, para reducir la frecuencia y la intensidad de los sofocos en 76 mujeres de Irán. Los resultados se expresaron como frecuencia diaria e intensidad media de los sofocos al final del tratamiento en la semana 8. No se especificó la medida de la intensidad.

La valeriana resultó significativamente más eficaz que placebo para Calidad reducir la frecuencia diaria (DME -2,92; $\mathrm{p}<0,001)$ y la intensidad de los muy baja sofocos $(\mathrm{DME}-4,63 ; \mathrm{p}<0,001)$ tras 8 semanas de tratamiento $(1 \mathrm{ECA}$, 8 semanas, 76 mujeres $)^{249}$.

\section{Resumen de la evidencia - fitoterapia}

\section{Extracto de isoflavonas de trébol rojo}

\begin{tabular}{c|c}
$\begin{array}{c}\text { Calidad } \\
\text { muy baja }\end{array}$ & $\begin{array}{l}\text { La evidencia sobre el efecto del extracto estandarizado de isoflavonas } \\
\text { de trébol rojo en relación a la reducción de la frecuencia de sofocos /día } \\
\text { /moderada }\end{array}$ \\
y sudores nocturnos y la mejora de la sintomatología de la menopausia
\end{tabular}




\begin{tabular}{|c|c|}
\hline & $\begin{array}{l}\text { en comparación a placebo no ha sido concluyente, encontrando estudios } \\
\text { a favor del tratamiento }{ }^{201} \text { y estudios en los que no existen diferencias } \\
\text { estadísticamente significativas }{ }^{120,261,262} \text {. }\end{array}$ \\
\hline $\begin{array}{l}\text { Calidad } \\
\text { muy baja/ } \\
\text { moderada }\end{array}$ & $\begin{array}{l}\text { No se han encontrado diferencias estadísticamente significativas entre el } \\
\text { extracto de isoflavonas de trébol rojo (Promensil®) y el placebo en la } \\
\text { proporción de mujeres que experimentaron algún efecto indeseado, ni } \\
\text { en la incidencia de infecciones del tracto respiratorio, cefalea, mialgias, } \\
\text { náuseas, artralgias, diarrea o pérdidas vaginales, así como en el grosor } \\
\text { endometrial (evaluado por ecografía) }{ }^{120} \text {. }\end{array}$ \\
\hline \multicolumn{2}{|c|}{ Isoflavonas de soja } \\
\hline $\begin{array}{l}\text { Calidad } \\
\text { muy baja }\end{array}$ & $\begin{array}{l}\text { Los resultados globales del metanálisis y los resultados por subgrupos } \\
\text { según el tipo de isoflavona considerado (en forma de suplementos } \\
\text { dietéticos, extractos o concentrados) han mostrado una diferencia } \\
\text { estandarizada media a favor de las isoflavonas de soja frente a placebo } \\
\text { en mujeres en la etapa de la peri o postmenopausia }{ }^{198} \text {. }\end{array}$ \\
\hline \multicolumn{2}{|c|}{ 1. Suplementos proteicos de soja } \\
\hline $\begin{array}{l}\text { Calidad } \\
\text { muy baja- } \\
\text { moderada }\end{array}$ & 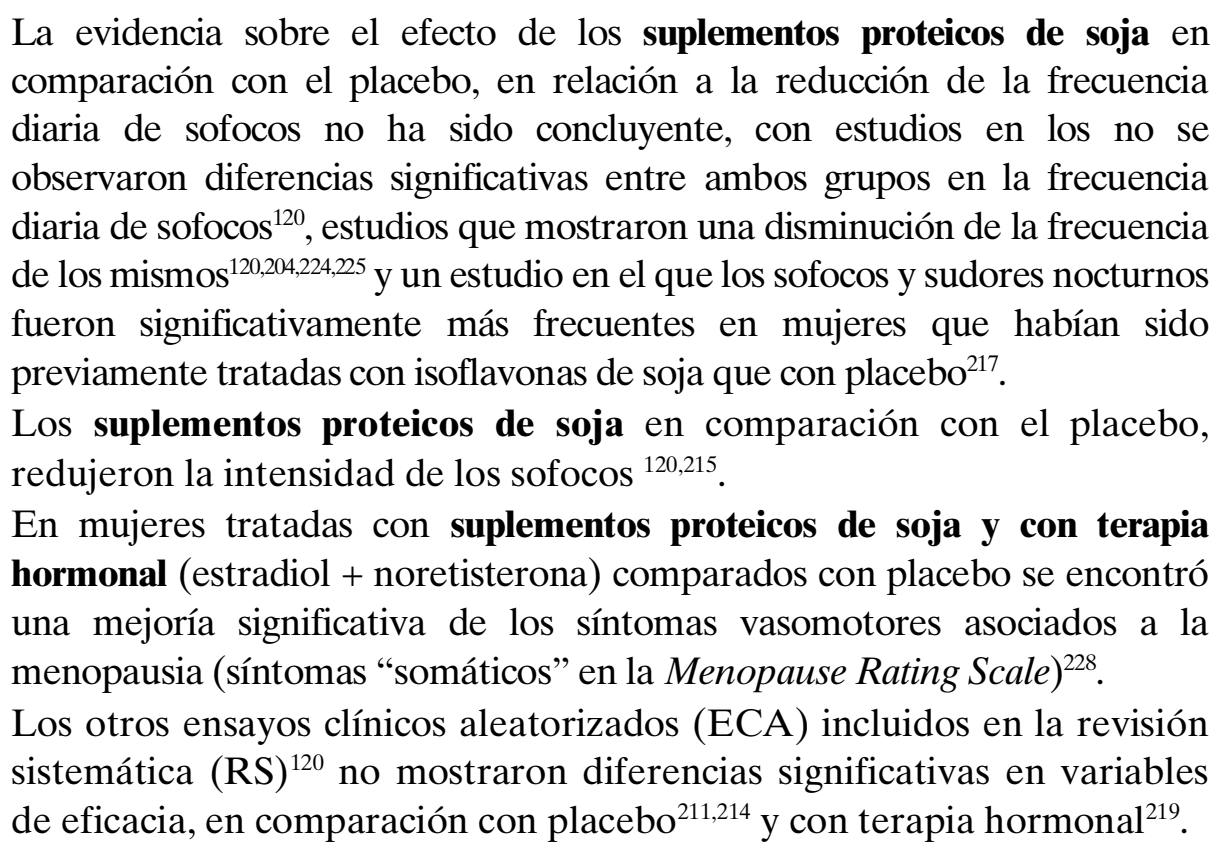 \\
\hline \multicolumn{2}{|c|}{ 2. Suplementos dietéticos de soja } \\
\hline $\begin{array}{l}\text { Calidad } \\
\text { muy baja- } \\
\text { baja- } \\
\text { moderada }\end{array}$ & $\begin{array}{l}\text { La evidencia en relación a los suplementos dietéticos de soja no es } \\
\text { concluyente, con resultados a favor de la reducción de la intensidad de } \\
\text { los sofocos con una dieta enriquecida en soja frente a una dieta normal } \\
\text { (mediante un cuestionario realizado a las mismas) }{ }^{120,207,224-226,228,229} \text {, con un }\end{array}$ \\
\hline
\end{tabular}




\begin{tabular}{|c|c|}
\hline & $\begin{array}{l}\text { producto lácteo rico en soja (asociado o no al ejercicio físico) }{ }^{120} \text { y } \\
\text { resultados similares entre el grupo tratado con suplementos dietéticos } \\
\text { de soja y el grupo control }{ }^{230,231232-236} \text {. }\end{array}$ \\
\hline \multicolumn{2}{|c|}{ 3. Extractos de isoflavonas de soja } \\
\hline $\begin{array}{l}\text { Calidad } \\
\text { baja o muy } \\
\text { baja }\end{array}$ & $\begin{array}{l}\text { El S-(-) equol (suplemento de isoflavonas de soja) se mostro superior de } \\
\text { forma estadísticamente significativa a placebo para reducir la frecuencia } \\
\text { diaria y la intensidad de sofocos, tras } 12 \text { semanas de tratamiento }{ }^{203} \text {. }\end{array}$ \\
\hline $\begin{array}{c}\text { Calidad } \\
\text { muy baja }\end{array}$ & $\begin{array}{l}\text { El S-equol (suplemento de isoflavonas de soja) no mostró diferencias } \\
\text { significativas frente a isoflavonas de soja (extracto) para reducir la frecuencia } \\
\text { diaria de sofocos, tras } 8 \text { semanas de tratamiento, independientemente de la } \\
\text { dosis }^{205} \text {. }\end{array}$ \\
\hline $\begin{array}{c}\text { Calidad } \\
\text { moderada }\end{array}$ & $\begin{array}{l}\text { Los extractos de isoflavonas de soja (naturales o sintéticos) se } \\
\text { mostraron significativamente más eficaces que placebo para reducir la } \\
\text { frecuencia y la gravedad (intensidad) de sofocos en mujeres en la etapa } \\
\text { de la peri y postmenopausia }{ }^{199} \text {. }\end{array}$ \\
\hline $\begin{array}{c}\text { Calidad } \\
\text { muy baja }\end{array}$ & $\begin{array}{l}\text { La terapia hormonal se mostró más eficaz que los extractos de isoflavonas } \\
\text { de soja (estandarizados o concentrados) para aliviar los sofocos (frecuencia } \\
\text { y gravedad) en mujeres en la etapa de la postmenopausia }{ }^{200} \text {. }\end{array}$ \\
\hline $\begin{array}{c}\text { Calidad } \\
\text { muy baja }\end{array}$ & $\begin{array}{l}\text { Las isoflavonas de soja (extracto) combinadas con erberena se mostraron } \\
\text { significativamente superiores a placebo para reducir la intensidad de los } \\
\text { sofocos y la sudoración nocturna en mujeres en climaterio con dislipemia, } \\
\text { tras } 12 \text { semanas de tratamiento }{ }^{202} \text {. }\end{array}$ \\
\hline $\begin{array}{c}\text { Calidad } \\
\text { muy baja }\end{array}$ & $\begin{array}{l}\text { Las isoflavonas de soja (germen) fueron significativamente superiores a } \\
\text { placebo para reducir la frecuencia semanal de sofocos y mejorar la } \\
\text { sintomatología asociada a la menopausia tras } 24 \text { semanas de tratamiento }{ }^{204} \text {. }\end{array}$ \\
\hline $\begin{array}{c}\text { Calidad } \\
\text { muy baja }\end{array}$ & $\begin{array}{l}\text { La combinación de isoflavonas de soja con Lactobacillus sporogenes no } \\
\text { mostró diferencias significativas frente a la misma combinación con } \\
\text { magnolia, para reducir la intensidad de los sofocos y la sudoración } \\
\text { nocturna tras } 12 \text { semanas de tratamiento }\end{array}$ \\
\hline \multicolumn{2}{|c|}{ 4. Concentrado de genisteína } \\
\hline $\begin{array}{l}\text { Calidad } \\
\text { muy baja- } \\
\text { moderada }\end{array}$ & $\begin{array}{l}\text { El tratamiento con un concentrado de genisteína ( } 54 \mathrm{mg} / \text { día) se asoció a } \\
\text { una disminución significativa del número e intensidad de los sofocos en } \\
\text { comparación con placebo a los } 12 \text { y } 24 \text { meses }^{222} \text {. } \\
\text { Los concentrados de genisteína ( } 30 \text { y } 60 \mathrm{mg} / \mathrm{d} \text { ) en comparación con } \\
\text { placebo redujeron significativamente la frecuencia diaria de sofocos } \\
\text { pero no la intensidad de los mismos }{ }^{120} \text {. }\end{array}$ \\
\hline
\end{tabular}




\begin{tabular}{|c|c|}
\hline $\begin{array}{l}\text { Calidad } \\
\text { muy baja- } \\
\text { moderada }\end{array}$ & $\begin{array}{l}\text { En mujeres tratadas con un concentrado de genisteína a dosis de } \\
54 \mathrm{mg} / \text { día en comparación a placebo, se observó una disminución del } \\
24 \% \text { en los sofocos diarios frente al placebo al año de seguimiento } \\
(\mathrm{p}<0,01) \text {. Comparando el tratamiento con un concentrado de } \\
\text { genisteína con el tratamiento hormonal, se observó una reducción del } \\
30 \% \text { a favor de la terapia hormonal }(\mathrm{p}<0,05)(1 \mathrm{ECA} ; 90 \text { mujeres })^{221} \text {. }\end{array}$ \\
\hline \multicolumn{2}{|c|}{ 5. Otros fitoestrógenos } \\
\hline $\begin{array}{l}\text { Calidad } \\
\text { baja o muy } \\
\text { baja }\end{array}$ & $\begin{array}{l}\text { Los extractos o suplementos dietéticos de linaza a diferentes dosis } \\
\text { diarias no mostraron diferencias significativas frente a placebo, en la } \\
\text { reducción de la frecuencia o la intensidad de los sofocos, de sudores } \\
\text { nocturnos y otra la sintomatología asociada a la menopausia, así como } \\
\text { en la reducción de la puntuación en la escala combinada de sofocos } \\
\text { (frecuencia e intensidad) tras } 6 \text { semanas de tratamiento }\end{array}$ \\
\hline $\begin{array}{l}\text { Calidad } \\
\text { baja o muy } \\
\text { baja }\end{array}$ & $\begin{array}{l}\text { Los extractos de lúpulo no mostraron diferencias significativas frente a } \\
\text { placebo en la intensidad de los sofocos (escala Kupperman })^{237} \text {. }\end{array}$ \\
\hline $\begin{array}{l}\text { Calidad } \\
\text { baja o muy } \\
\text { baja }\end{array}$ & $\begin{array}{l}\text { El extracto ERr } 731 \text { de ruibarbo (Rheum rhaponticum) comparado con } \\
\text { placebo ha mostrado una reducción significativa de la intensidad y la } \\
\text { frecuencia de sofocos y sudores nocturnos }{ }^{238} \text {. }\end{array}$ \\
\hline $\begin{array}{c}\text { Calidad } \\
\text { muy baja }\end{array}$ & $\begin{array}{l}\text { La incidencia global de efectos indeseados de los fitoestrógenos fue } \\
\text { similar a la del grupo control, salvo en el caso de los efectos } \\
\text { gastrointestinales que fueron más frecuentes en el grupo tratado con } \\
\text { fitoestrógenos }{ }^{239} \text {. }\end{array}$ \\
\hline $\begin{array}{c}\text { Calidad } \\
\text { muy baja }\end{array}$ & $\begin{array}{l}\text { Se ha observado una reducción estadísticamente significativa del riesgo } \\
\text { de cáncer de mama en el grupo con un elevado consumo de lignanos, si } \\
\text { bien, esta reducción de riesgo fue significativa en el subgrupo de } \\
\text { mujeres en la etapa de la postmenopausia }{ }^{240} \text {. }\end{array}$ \\
\hline $\begin{array}{c}\text { Calidad } \\
\text { moderada/ } \\
\text { muy baja }\end{array}$ & $\begin{array}{l}\text { Cimicífuga racemosa no ha mostrado eficacia en el tratamiento de los } \\
\text { síntomas vasomotores asociados a la menopausia }{ }^{241} \text {, aunque un estudio } \\
\text { ha mostrado la eficacia de Cimicífuga racemosa en la reducción de } \\
\text { frecuencia e intensidad de sofocos y otros síntomas asociados a la } \\
\text { menopausia, tras } 12 \text { semanas de tratamiento }{ }^{244} \text {. }\end{array}$ \\
\hline $\begin{array}{c}\text { Calidad } \\
\text { BAJA o } \\
\text { MUY BAJA }\end{array}$ & $\begin{array}{l}\text { Se han descrito casos aislados de toxicidad hepática tras el tratamiento con } \\
\text { Cimifuga Racemosa pero no se ha podido establecer una clara relación de } \\
\text { causalidad }{ }^{243,263} \text {. La AEMPS publicó una alerta desaconsejando su uso en } \\
\text { las mujeres con antecedentes de enfermedad hepática o en las que } \\
\text { siguiendo un tratamiento con Cimicífuga racemosa experimenten signos o } \\
\text { síntomas de alteración de la función hepática como astenia, náuseas, } \\
\text { coluria e ictericiaa }\end{array}$ \\
\hline
\end{tabular}




\begin{tabular}{|c|c|}
\hline \multicolumn{2}{|c|}{ 6. Otras hierbas medicinales } \\
\hline $\begin{array}{c}\text { Calidad } \\
\text { BAJA }\end{array}$ & $\begin{array}{l}\text { La hierba de San Juan ha mostrado efectos discretos en los síntomas } \\
\text { vasomotores de las mujeres durante la postmenopausia sin antecedentes } \\
\text { de cáncer de mama (número e intensidad de los sofocos) } \text { ) }^{251,252,264} \text {. }\end{array}$ \\
\hline $\begin{array}{c}\text { Calidad } \\
\text { moderada }\end{array}$ & $\begin{array}{l}\text { Se han descrito posibles interacciones de hipérico con varios fármacos } \\
\text { metabolizados por un enzima hepático (citocromo P-450) como } \\
\text { carbamacepina, ciclosporina, anticonceptivos orales, nifedipina, etc., así } \\
\text { como el riesgo de padecer un síndrome serotoninérgico al tomarse } \\
\text { conjuntamente con antidepresivos }{ }^{253-256} \text {. }\end{array}$ \\
\hline $\begin{array}{c}\text { Calidad } \\
\text { baja }\end{array}$ & $\begin{array}{l}\text { La combinación de Hierba de San Juan con Cimifuga Racemosa } \\
\text { mostró una reducción estadísticamente significativa de síntomas } \\
\text { asociados a la menopausia (MRS: Menopause Rating Scale) en } \\
\text { comparación con placebo }{ }^{252} \text {. }\end{array}$ \\
\hline $\begin{array}{c}\text { Calidad } \\
\text { muy baja }\end{array}$ & $\begin{array}{l}\text { Se ha encontrado una disminución de los síntomas asociados a la } \\
\text { menopausia, medidos según diferentes escalas combinadas, en mujeres } \\
\text { tratadas con Lepidium meyenii }{ }^{252,258,259} \text {, con Panax ginseng }{ }^{246} \text {, con } \\
\text { Pimpinella anisum }{ }^{247} \text {, con regaliz ("Liquorice") }{ }^{248} \text { y con valeriana }{ }^{249} \text {, en } \\
\text { comparación con placebo. Igualmente, el tratamiento combinado chino } \\
\text { de hierbas conocido como la "decocción ER" ha mostrado una } \\
\text { disminución de los síntomas asociados a la menopausia en comparación } \\
\text { con tratamientos no hormonales, pero no comparando con tratamientos } \\
\text { hormonales. }\end{array}$ \\
\hline
\end{tabular}

\section{Recomendaciones}

\begin{tabular}{|c|l|}
\hline DÉBIL & $\begin{array}{l}\text { En mujeres en etapa peri y postmenopáusica que no realizan } \\
\text { tratamiento hormonal (o que éste está contraindicado) y que } \\
\text { presentan síntomas vasomotores que afectan a su calidad de vida, } \\
\text { se sugiere valorar el tratamiento con fitoestrógenos (extractos } \\
\text { naturales o sintéticos de soja, concentrados de genisteína* o } \\
\text { suplementos dietéticos de soja). En la elección del preparado, } \\
\text { debe considerarse y conocerse la composición así como la dosis } \\
\text { empleada. }\end{array}$ \\
\hline DÉBIL & $\begin{array}{l}\text { Se sugiere no utilizar el tratamiento con otros fitoestrógenos, Cimifuga } \\
\text { Racemosa y otros tratamientos de hierbas medicinales en mujeres con } \\
\text { síntomas vasomotores en etapa peri y postmenopáusica. }\end{array}$ \\
\hline
\end{tabular}




\begin{tabular}{|l|l|}
\hline FUERTE & $\begin{array}{l}\text { No se debe administrar Cimifuga Racemosa a aquellas mujeres } \\
\text { con enfermedad hepática conocida o en tratamiento con fármacos } \\
\text { hepatotóxicos. }\end{array}$ \\
\hline
\end{tabular}

* Los concentrados de genisteína no se encuentran comercializados en España. 


\subsection{Intervenciones no farmacológicas}

\subsubsection{Acupuntura}

\subsubsection{Acupuntura tradicional frente a placebo (acupuntura simulada)}

Una RS (6 ECA, 309 participantes) evaluó la eficacia de la acupuntura en el tratamiento de los sofocos en mujeres perimenopáusicas en comparación con acupuntura simulada ${ }^{265}$.

La eficacia de la acupuntura en la frecuencia de sofocos se evaluó en tres ECA (125 participantes).

En un ECA más reciente (51 mujeres en etapa postmenopáusica) se comparó el efecto de la acupuntura tradicional china frente a placebo (acupuntura simulada) sobre los síntomas vasomotores tras 12 semanas de tratamiento ${ }^{266}$.

Otro ECA ( $\mathrm{n}=55$ mujeres en etapa postmenopáusica) comparó la eficacia de la acupuntura tradicional china frente a acupuntura simulada sobre diversos síntomas asociados a la menopausia después de 10 sesiones de tratamiento ( 5 semanas $)^{267}$.

En un ECA piloto para determinar la viabilidad de realizar un ensayo clínico posterior más amplio, se seleccionaron 60 mujeres en etapas peri y postmenopáusica (de las que sólo 33 completaron el estudio) que se distribuyeron en tres grupos: acupuntura tradicional, acupuntura simulada y grupo control ${ }^{268}$.

La eficacia de la acupuntura en relación a la frecuencia de sofocos no Calidad baja mostró diferencias entre las intervenciones (3 ECAs, 125 mujeres).

No se encontraron diferencias significativas entre los grupos, ya que Calidad en ambos casos se observó una mejora de los sofocos, tanto en su muy baja frecuencia (que disminuyó una media de 2,6 con acupuntura real frente a 2,5 con placebo) como en la puntuación de escala de síntomas (disminución media de 2,4 en ambos grupos) (1 ECA, 51 mujeres) ${ }^{266}$.

La intensidad de los sofocos se evaluó en dos ECA (76 participantes) Calidad baja mediante una escala analógica visual: un estudio no mostró diferencias entre los dos grupos mientras que en el otro, el grupo acupuntura mostró una reducción significativa respecto a los valores basales pero no frente a la acupuntura simulada. No se compararon estadísticamente los grupos de intervención.

Otro estudio encontró que la gravedad de los sofocos (utilizando la escala Menopause Rating Scale - MRS) disminuyó significativamente en el grupo tratado con acupuntura real una media de 3,2 $\pm 2,1$ frente a $8,8 \pm 2,3$ $(p=0,001)$ en el grupo control ${ }^{267}$.

La evaluación de los sofocos mediante un índice que incluyó Calidad baja frecuencia e intensidad fue analizado como variable en tres ECA (188 participantes): en dos estudios no hubo diferencias entre los grupos en los valores finales o en el cambio respecto al basal; en un tercer estudio 
el grupo acupuntura se mostró superior a la acupuntura simulada en la reducción respecto al basal del índice de intensidad pero no para el índice de frecuencia.

Tras 12 semanas de tratamiento los resultados mostraron una mayor Calidad mejoría de los síntomas vasomotores en los grupos de acupuntura muy baja tradicional y simulada comparados frente al grupo control $(\Delta-3,5 \pm 3,00$ vs. $-4,1 \pm 3,79$ vs. $-1,2 \pm 2,4$; respectivamente; $\mathrm{p}=0,20)$ y una mejora significativa de los síntomas vasomotores según el cuestionario MENQOL $(\Delta-1,5 \pm 2,02 \text { vs. }-1,8 \pm 1,52 \text { vs. } 0,3 \pm 0,64 \text {; respectivamente; } \mathrm{p}=0,04)^{268}$.

Los efectos indeseados fueron poco frecuentes y de escasa intensidad Calidad baja en los dos grupos de intervención.

\subsubsection{Acupuntura tradicional frente a otros tratamientos}

Una RS muy similar evaluó los mismos estudios que la anterior y añadió Calidad baja cinco estudios adicionales en los que la acupuntura se comparó con un tratamiento activo (terapia hormonal o fitoestrógenos) ${ }^{269}$. Los estudios presentaron limitaciones en el diseño y la ejecución.

Los cinco estudios adicionales incluyeron un total de 455 participantes, fueron realizados en China y la valoración de la eficacia se realizó a través de la escala de Kupperman. En dos de los tres estudios la acupuntura se mostró significativamente superior a la terapia hormonal mientras que un tercero no observó diferencias entre las intervenciones.

Los resultados no mostraron diferencias significativas entre los Calidad grupos, ya que en ambos casos se mejoraron los sofocos, tanto en su muy baja frecuencia (que disminuyó una media de 2,6 con acupuntura real frente a 2,5 con placebo) como en la puntuación de escala de síntomas (disminución media de 2,4 en ambos grupos) ${ }^{266}$.

Un ECA posterior (167 participantes) de 12 semanas de duración Calidad baja que comparó 10 sesiones de acupuntura más consejos de autocuidado frente a consejos de autocuidado solo ${ }^{270}$, mostró mejores resultados para la acupuntura que para el grupo control con una disminución mayor en la frecuencia media diaria de los sofocos $(-5,8 v s$. - 3,7) y de la intensidad de los mismos (3,2 unidades vs. 1,8), diferencias ambas estadísticamente significativas, pero el estudio presentaba importantes limitaciones metodológicas (no comparación con acupuntura simulada y no ciego).

La moxibustión es una técnica tradicional china consistente en la Calidad baja aplicación de calor generado por la combustión de un principio botánico (Artemisa vulgaris) en los puntos de acupuntura. Un ECA (51 mujeres) de cuatro semanas de duración mostró una reducción de la frecuencia y la intensidad de los sofocos con moxibustión en comparación con control (lista de espera) $)^{271}$. 


\subsubsection{Otros tipos de acupuntura}

En un ECA (40 mujeres en etapas peri y postmenopáusica) se comparó el Calidad efecto de acupuntura láser frente a acupuntura simulada sobre la muybaja reducción de la frecuencia de los sofocos (diurnos y nocturnos), mediante sesiones quincenales durante 12 semanas ${ }^{272}$.

Al final del tratamiento, el número de sofocos diurnos por semana disminuyó una media de 33,6 \% en el grupo placebo y 36,6 \% en el grupo de intervención; y en cuanto a la reducción en la frecuencia de los sofocos nocturnos la media fue de $30,0 \%$ y $29,8 \%$ respectivamente. Aunque las reducciones fueron significativas con respecto a los valores basales, no hubo diferencias significativas en cuanto a los cambios observados entre el placebo y el tratamiento activo ${ }^{272}$.

En otro ECA ( $\mathrm{n}=54$ mujeres en etapas peri y postmenopáusica) se Calidad comparó el efecto de la acupuntura koreana frente a acupuntura simulada muy baja (7 semanas de tratamiento) sobre los sofocos, evaluando también el seguimiento a las 11 semanas $^{273}$.

Se evaluaron como variables principales la escala de sofocos, la frecuencia y la gravedad de los mismos, no detectándose diferencias significativas entre los grupos, excepto la gravedad de los sofocos a las 7 semanas, en la que se obtuvieron reducciones medias de $-1,0 \pm 0,9$ en el grupo sometido a acupuntura real y $-0,4 \pm 0,6$ en el grupo placebo $(p=0,0150)$.

En un pequeño ECA $(n=46)$ realizado en menopáusicas ovariectomizadas Calidad se comparó el efecto de acupuntura china+digitopresión auricular frente a muy baja tibolona oral durante 12 semanas, realizando un seguimiento 4 semanas después de finalizar el tratamiento ${ }^{274}$.

En todos los grupos la frecuencia e intensidad de los sofocos se redujo significativamente con respecto a los valores obtenidos antes del tratamiento, y se obtuvieron mejores resultados de eficacia en el grupo tratado con tibolona. Así, a las 12 semanas de tratamiento se obsevó que la diferencia no fue significativa en cuanto a la gravedad de los sofocos entre el grupo de la intervención $(3,86 \pm 0,84)$ y el de tibolona $(3,71 \pm 0,65 ; \mathrm{p}>0,05)$; sin embargo la reducción de los sofocos fue significativamente menor en el grupo de la intervención $(10,32 \pm 3,13)$ que en el grupo tratado con tibolona $(7,48 \pm 2,69 ; \mathrm{p}<0,05)$.

\subsubsection{Terapias dirigidas a la educación en la patología}

\subsubsection{Intervenciones psicológicas y educacionales}

Una RS evaluó las intervenciones psicoeducacionales para los síntomas Calidad baja vasomotores $^{275}$ e incluyó dos ECA que evaluaron la eficacia de la terapia cognitivo-conductual en mujeres durante la postmenopausia sin historia de cáncer de mama ${ }^{276,277}$. El primero de ellos aleatorizó 73 mujeres a recibir tratamiento hormonal con o sin terapia cognitivo-conductual adicional. 
$\mathrm{Al}$ final del seguimiento de seis meses ambos grupos experimentaron una reducción de los síntomas vasomotores (escala de Kupperman) siendo más importante la reducción en el grupo que recibió ambas intervenciones ${ }^{277}$. El segundo estudio incluyó a 19 mujeres durante la postmenopausia (sin tratamiento hormonal) a recibir tratamiento cognitivo-conductual de forma inmediata o diferida durante ocho semanas. Dos de las ocho mujeres que recibieron la intervención diferida abandonaron el estudio (25\%). Sólo el grupo de intervención inmediata presentó una reducción significativa de los síntomas vasomotores aunque no se compararon los dos grupos directamente ${ }^{276}$.

Un ECA más reciente (110 mujeres con sofocos y sudoraciones Calidad nocturnas) comparó la eficacia de la Reducción del Estrés Basada en la muy baja Atención Plena (Mindfulness-Based Stress Reduction - MBSR) frente a un grupo control para reducir el grado de molestia de los sofocos ${ }^{278}$. El MBSR es un programa estructurado que utiliza técnicas orientadas para aliviar el dolor y mejorar el bienestar físico y emocional ante un determinado trastorno o enfermedad y en este estudio se ofrecieron clases semanales de 2,5 horas durante 8 semanas, realizando un seguimiento durante 3 meses adicionales (total: 20 semanas). La variable principal incluía tanto sofocos como sudoraciones nocturnas y la intensidad diurna de los sofocos fue considerada como variable secundaria.

Los cambios observados en las molestias debidas a los sofocos mostraron diferencias significativas por brazo de tratamiento (interacción semana $\mathrm{x}$ brazo de tratamiento $\mathrm{p}=0,042)$. Al finalizar el tratamiento ( $9^{\mathrm{a}}$ semana) las molestias disminuyeron un $14,77 \%$ en el grupo de la intervención frente a $6,79 \%$ en el grupo control. A las 20 semanas la reducción total fue de $21,62 \%$ en el grupo de la intervención vs. $10,50 \%$ en el control. Los cambios ajustados para las condiciones iniciales en cuanto a la intensidad de los sofocos no difirieron entre los brazos de tratamiento (interacción semana $\mathrm{x}$ brazo de tratamiento $\mathrm{p}=0,692$ ).

\subsubsection{Intervenciones de entrenamiento en relajación}

Se ha localizado una RS reciente que evaluó las terapias de relajación en mujeres durante la postmenopausia con o sin cáncer de mama. Un total de cinco ECAs incluyeron mujeres sin cáncer de mama, en tres de ellos el comparador fue placebo. Las limitaciones metodológicas, en su conjunto, fueron importantes y las técnicas, los comparadores y el tiempo de seguimiento difirieron de forma considerable entre los estudios ${ }^{279}$.

En un ECA abierto ( $\mathrm{n}=60$ en etapa postmenopáusica) se evaluó, frente a un grupo control, la eficacia de la relajación aplicada (10 sesiones durante 12 semanas) que es una técnica basada en la terapia cognitivo-conductual, enfocada a la relajación muscular, utilizando la respiración para favorecer la relajación ${ }^{280}$.

De los tres estudios frente a placebo incluidos en la RS identificada, Calidad baja un estudio mostró una reducción de la severidad de los sofocos del 21,9\% para la técnica de relajación y del $9 \%$ en el grupo control (lista de 
espera). No se compararon estadísticamente los resultados ${ }^{281}$. Otro ECA (33 mujeres) evaluó la frecuencia de sofocos con una monitorización de 24 horas usando un transductor cutáneo y mostró una reducción significativa a las cuatro semanas del 38,9 \% para el grupo de relajación (control de la respiración) en comparación con el grupo de entrenamiento a la relajación muscular $(4,2 \%)$ y el grupo control (aumento del $16,5 \%)^{282}$. En un último estudio (27 mujeres), la intervención (relajación con sonidos de baja frecuencia) mostró una reducción en el número y frecuencia de síntomas en comparación con control a las dos semanas ${ }^{283}$.

Dos estudios compararon técnicas de relajación frente a una intervención activa. Un ECA (28 mujeres en etapa postmenopáusica) mostró una reducción significativa de la frecuencia de sofocos favorable a la relajación $(54,5 \%)$ frente a técnicas de retroalimentación (incremento de $18,6 \%)$ a las seis semanas ${ }^{284}$. Un segundo ECA (58 mujeres) mostró una mayor reducción de los síntomas vasomotores valorados a través del índice de Kupperman (76,5\% frente a $41,9 \%$ ) y de una escala analógica visual $(72,7 \%$ frente a $57,8 \%)$ en el grupo de tratamiento hormonal en comparación con el grupo que realizó técnicas de relajación ${ }^{285}$.

A las 12 semanas de tratamiento, en el grupo de mujeres sometidas a Calidad relajación aplicada (10 sesiones durante 12 semanas), la frecuencia diaria muy baja de sofocos disminuyó 5,0 puntos frente a 1,9 en el grupo control $(\mathrm{p}<0,001)$, disminución que se mantuvo a los 3 meses de seguimiento $(\mathrm{p}<0,001)$. La reducción de los sofocos en el grupo de la intervención fue del $55 \%$ (semana 12 ) y del $52 \%$ (a los 3 meses de seguimiento), mientras que en el grupo control fue de $20 \%$ y $18 \%$ respectivamente ${ }^{280}$.

\subsubsection{Otros tratamientos}

\subsubsection{Reflexología}

Un ECA (69 mujeres) comparó la reflexología podal frente al masaje Calidad inespecífico en los pies no mostrando diferencias significativas entre los muy baja dos grupos en cuanto a la intensidad de los sofocos y las sudoraciones nocturnas a las 19 semanas $^{286}$.

\subsubsection{Aromaterapia}

Un estudio abierto que incluyó 60 mujeres durante la postmenopausia Calidad asignó de forma probablemente no aleatoria a recibir masajes de muybaja aromaterapia o control (lista de espera) durante 8 semanas ( 8 sesiones). Un total de ocho mujeres no completaron el estudio y se excluyeron del análisis $(13,3 \%)$. A las ocho semanas el grupo que recibió aromaterapia mostró una puntuación global de la escala de Kupperman estadísticamente inferior (mostrando mejoría) al grupo control ${ }^{287}$. 


\subsubsection{Vitamina $E$}

Un ECA (51 mujeres) evaluó la eficacia de los suplementos de Vitamina E Calidad (400 UI/día) para el tratamiento de los sofocos en mujeres en etapa muy baja postmenopáusica. Se evaluó la intensidad y la frecuencia de los sofocos con una escala analógica visual ( 0 a 10$)$. El tratamiento con Vitamina E redujo la frecuencia $(3,19 \pm 2,74$ frente a $5 \pm 3,34)$ y la intensidad $(1,8 \pm 0,87$ frente a $2,37 \pm 0,74)$ de los sofocos en comparación con placebo ${ }^{288}$.

\subsubsection{Suplementos de ácidos grasos omega-3}

Un ECA evaluó el beneficio de administrar suplementos de ácidos Calidad grasos omega-3 durante ocho semanas, en 91 mujeres de mediana edad muy baja (40 a 50 años) con sofocos. El estudio mostró una diferencia significativa en la reducción del número de sofocos al día respecto placebo $-1,58$ (IC $95 \%-2,18$ a $-0,98$ ) pero no en la reducción de la intensidad 0,50 (IC $95 \%-1,20$ a 0,20$)^{289}$.

\subsubsection{Suplementos de aminoácidos}

Un ECA incluyó a 100 mujeres en etapa postmenopáusica a recibir Calidad suplementos de isoleucina (5 g/día, 10 cápsulas diarias) o placebo durante muy baja 12 semanas. La reducción relativa de la frecuencia e intensidad de los sofocos fue superior en el grupo placebo aunque no de forma significativa ${ }^{290}$.

\subsubsection{Suplementos de micronutrientes}

Un ECA piloto realizado en 54 mujeres en etapa postmenopáusica asignó Calidad a recibir una cápsula diaria de micronutrientes (vitaminas y minerales) o muy baja placebo durante 3 meses para evaluar la eficacia sobre diversos síntomas menopáusicos, entre ellos sofocos y sudoración nocturna ${ }^{291}$.

Un $65 \%$ de las mujeres del grupo tratado con micronutrientes mostró una mejora casi completa de los sofocos a los 90 días de tratamiento frente a un $15 \%$ del grupo placebo $(\mathrm{p}=0,001)$. En cuanto a las sudoraciones nocturnas, a los 60 días de tratamiento el 85,7\% de las mujeres en el grupo de la intervención mostró una mejora de moderada a buena frente a un $50 \%$ en el grupo placebo $(\mathrm{p}=0,04)$.

\section{Resumen de la evidencia - tratamientos no farmacológicos}

\begin{tabular}{|c|c|}
\hline $\begin{array}{c}\text { Calidad } \\
\text { MUY } \\
\text { BAJA/BAJA }\end{array}$ & $\begin{array}{l}\text { Existe evidencia en cuanto a los potenciales beneficios de la } \\
\text { acupuntura sobre la frecuencia e intensidad de los síntomas } \\
\text { vasomotores de mujeres en etapa postmenopáusica, aunque ésta no } \\
\text { es concluyente }^{265-267,269-274} \text {. }\end{array}$ \\
\hline
\end{tabular}




\begin{tabular}{|c|c|}
\hline $\begin{array}{c}\text { Calidad } \\
\text { MUY } \\
\text { BAJA/BAJA }\end{array}$ & $\begin{array}{l}\text { Existe evidencia sobre los potenciales beneficios de la educación } \\
\text { (como la terapia cognitivo-conductual y la reducción del estrés } \\
\text { basada en la atención plena) sobre la reducción de los síntomas } \\
\text { vasomotores de mujeres en etapa postmenopáusica, si bien no es } \\
\text { concluyente }{ }^{275-277,279,281-285} \text {. }\end{array}$ \\
\hline $\begin{array}{c}\text { Calidad } \\
\text { MUY BAJA }\end{array}$ & $\begin{array}{l}\text { La eficacia de otras terapias alternativas (reflexología }{ }^{286} \text {, aromaterapia }{ }^{287} \text {, } \\
\text { vitamina } \mathrm{E}^{288} \text {, omega- } 3^{289} \mathrm{o} \text { aminoácidos }{ }^{290} \mathrm{o} \text { suplementos con aminoácidos }{ }^{290} \\
\text { o micronutrientes }{ }^{291} \text { ) sobre los síntomas vasomotores de mujeres en } \\
\text { etapa postmenopáusica es muy incierta. }\end{array}$ \\
\hline
\end{tabular}

\section{Recomendaciones}

\begin{tabular}{|l|l|}
\hline DÉBIL & $\begin{array}{l}\text { En mujeres con síntomas vasomotores durante la etapa de la peri y } \\
\text { postmenopausia, se sugiere valorar como tratamientos complementarios } \\
\text { la acupuntura o las intervenciones educativas (terapia cognitivo- } \\
\text { conductual y la reducción del estrés basada en la atención plena) sobre } \\
\text { los síntomas vasomotores. }\end{array}$ \\
\hline
\end{tabular}





\section{Tratamiento de los síntomas vaginales}

\section{Preguntas para responder}

En mujeres con síntomas vaginales durante la peri y postmenopausia,

- ¿Cuál es la eficacia y la seguridad de la modificación de los estilos de vida?

- ¿Cuál es la eficacia y la seguridad de los estrógenos?

- ¿Cuál es la eficacia y la seguridad de los gestágenos?

- ¿Cuál es la eficacia y la seguridad de la tibolona?

- ¿Cuál es la eficacia y la seguridad de la fitoterapia?

- ¿Cuál es la eficacia y la seguridad de los moduladores selectivos de los receptores estrogénicos (SERM)?

- ¿Cuál es la eficacia y la seguridad de los lubricantes e hidratantes vaginales?

La atrofia vaginal se asocia con la menopausia y su prevalencia aumenta en la transición de la premenopausia a la postmenopausia ${ }^{7}$. En el estudio $\mathrm{WHI}^{9}$ se constataron las siguientes frecuencias de diversos síntomas vaginales como: sequedad $(27,0 \%)$, irritación o picor $(18,6 \%)$, flujo $(11,1 \%)^{292}$. Estos síntomas pueden tener un impacto significativo en la calidad de vida y la actividad sexual, por la dispareunia que genera la sequedad vaginal, pudiendo ocasionar una pérdida de interés sexual ${ }^{293}$.

En este apartado se revisan las distintas intervenciones propuestas para mejorar los síntomas vaginales.

\subsection{Intervenciones en los estilos de vida}

El estudio observacional 'Study of Women's Health Across the Nation ${ }^{\text {,294 }}$ ha Calidad baja mostrado una asociación entre determinados factores de riesgo y estilos de vida con los síntomas vaginales. Algunos determinantes sociales identificados como factores de riesgo (origen cultural, edad, factores socioeconómicos, etc.) no son modificables, mientras que otros (estilos de vida o IMC) sí son susceptibles de ser sometidos a unas estrategias de prevención.

Este estudio mostró que no realizar ejercicio físico se asocia con un riesgo mayor de padecer síntomas vaginales (OR 1,64; IC $95 \%$ 1,29 a 2,08), en comparación con realizar ejercicio físico intenso. El IMC $>27 \mathrm{~kg} / \mathrm{m}^{2}$ también se ha asociado con la presencia de sequedad vaginal. Por el contrario, el tabaco no ha mostrado una asociación significativa con la presencia de atrofia vaginal ${ }^{294}$. 
Resumen de la evidencia - intervenciones en los estilos de vida

\begin{tabular}{|c|l|}
\hline \multicolumn{2}{|c|}{ Ejercicio físico } \\
\hline $\begin{array}{c}\text { Calidad } \\
\text { BAJA }\end{array}$ & $\begin{array}{l}\text { No realizar ejercicio físico se asocia con un mayor riesgo de } \\
\text { presentar síntomas vaginales }\end{array}$ \\
\hline \multicolumn{2}{|c|}{ Obesidad } \\
\hline $\begin{array}{c}\text { Calidad } \\
\text { BAJA }\end{array}$ & $\begin{array}{l}\text { La obesidad se asocia a un mayor riesgo de presentar síntomas } \\
\text { vaginales }\end{array}{ }^{294}$. \\
\hline \multicolumn{1}{|c|}{ Tabaco } \\
\hline $\begin{array}{l}\text { Calidad } \\
\text { BAJA }\end{array}$ & $\begin{array}{l}\text { El tabaquismo no se asocia a un mayor riesgo de presentar } \\
\text { síntomas vaginales }\end{array}$ \\
\hline
\end{tabular}

\section{Recomendaciones}

\begin{tabular}{|l|l|}
\hline FUERTE & $\begin{array}{l}\text { En mujeres durante la peri y postmenopausia se recomienda evitar la } \\
\text { obesidad y realizar ejercicio físico para prevenir la aparición de } \\
\text { síntomas vaginales. }\end{array}$ \\
\hline
\end{tabular}

\subsection{Intervenciones farmacológicas}

\subsubsection{Tratamiento hormonal}

\subsubsection{Estrógenos}

Una RS (19 ECA, 4.162 mujeres) comparó la efectividad, seguridad y aceptabilidad de preparaciones estrogénicas locales para mujeres con atrofia vaginal ${ }^{295}$. Los ECA incluidos midieron variables distintas, fueron de pequeño tamaño y mostraron heterogeneidad significativa en los resultados, por lo que sus resultados deben interpretarse con precaución. Además se identificaron dos estudios ${ }^{296,297}$ que evaluaron el efecto de los estrógenos orales en mujeres en la etapa de la postmenopausia sobre la atrofia vaginal y dos estudios posteriores a la revisión sistemática ${ }^{298,299}$ que evaluaron el efecto de los estrógenos en comprimidos vaginales en comparación con los estrógenos en crema sobre la sequedad, dolor e irritación vaginales. Un ECA evaluó los efectos de los estrógenos transdérmicos frente a placebo $^{300}$ para el alivio de los síntomas vulvovaginales en mujeres durante la etapa de la postmenopausia. 
Se han identificado además tres estudios que evaluaron la eficacia de los estrógenos en el tratamiento de los síntomas vaginales asociados a la menopausia: un análisis secundario del estudio Women's Health Initiative (WHI) sobre estrógenos orales ${ }^{85} \mathrm{y}$ tres ECA (dos publicados de forma conjunta) sobre estrógenos transdérmicos ${ }^{95,301}$.

\subsection{Estrógenos orales frente a placebo}

Dos estudios ${ }^{296,297}$ evaluaron el efecto de los estrógenos orales frente a placebo en mujeres en la etapa de la postmenopausia sobre la atrofia vaginal.

Además, el análisis secundario del estudio WHI 1998 (10.739 mujeres) ${ }^{302}$, evaluó la eficacia de los estrógenos conjugados equinos (ECC $0,625 \mathrm{mg} / \mathrm{d}$ ) para reducir los sofocos y sudores nocturnos frente a placebo, en mujeres histerectomizadas en la etapa de la postmenopausia, tras un año de tratamiento. También analizó el efecto del tratamiento sobre la sequedad vaginal y otros síntomas asociados a la menopausia. De forma complementaria, se realizó un seguimiento (estudio de cohorte) de las mujeres que continuaron con el tratamiento hormonal tras la finalización del estudio WHI, analizando la evolución de la sintomatología, los efectos de la suspensión del mismo y los posibles factores influyentes en su evolución. Los resultados se expresaron como la proporción de mujeres que presentaron cada uno de los síntomas o efectos analizados tras un año de tratamiento, calculados en función de la presencia o ausencia de sintomatología basal; lo que dificultó la realización de comparaciones con otros estudios. La metodología no se especificó de forma detallada al tratarse de un análisis secundario de otro estudio.

Dos estudios con estrógenos orales para la atrofia vaginal ${ }^{296,297}$ mostraron mejorías significativas en la sequedad vaginal y en la intensidad de los síntomas, respectivamente, en comparación con placebo.

El análisis secundario del estudio $\mathrm{WHI}^{302}$ mostró que los estrógenos equinos conjugados $(0,625 \mathrm{mg} / \mathrm{d})$ fueron significativamente más eficaces que el placebo $(\mathrm{p}<0,001)$ para reducir la proporción de mujeres con sequedad vaginal tras un año de tratamiento (1 estudio, 1 año, 10.739 mujeres):

- Mujeres sin sintomatología basal: RR 0,79; IC 95 \% 0,65 a 0,96.

- Mujeres con sintomatología basal: RR 0,80; IC 95 \% 0,68 a 0,93.

\subsection{Estrógenos en comprimidos vaginales frente a placebo}

Dos ensayos clínicos evaluaron la eficacia de dosis bajas y ultrabajas de estradiol vaginal en comprimidos para el tratamiento de la vaginitis atrófica frente a placebo ${ }^{297,299}$.

Estos estudios mostraron una mejoría significativa de los síntomas vaginales (sequedad, dolor e irritación) con los estrógenos en comprimidos en comparación con placebo ${ }^{297,299}$. Así mismo se observó

\section{Calidad} muy baja un caso de hiperplasia endometrial con los comprimidos vaginales de 17beta-estradiol $^{299}$ y dos sangrados vaginales con estrógenos conjugados $^{297}$ (no estadísticamente significativos).

\section{Calidad moderada}


También se observaron diferencias significativas en el sangrado vaginal a favor del placebo en un ECA que comparó comprimidos con placebo (1.612 mujeres, OR 10,48; IC $95 \%$ 0,58 a 189,92).

\subsection{Estrógenos en óvulos frente a estrógenos en crema}

Dos estudios posteriores a la revisión sistemática ${ }^{298,299}$ evaluaron el efecto de los estrógenos en comprimidos vaginales en comparación con los estrógenos en crema sobre la sequedad, el dolor e irritación vaginales.

Estos estudios mostraron una mejoría significativa de los síntomas (sequedad, dolor e irritación) con los estrógenos en comprimidos vaginales (óvulos) en comparación con los estrógenos en crema ${ }^{298,299}$.

Se observaron casos no estadísticamente significativos de hiperplasia endometrial con el anillo, la crema y los comprimidos vaginales (con 17beta-estradiol).

\section{Calidad moderada}

\section{Calidad moderada}

\subsection{Estrógenos en gel vaginal frente a placebo}

Uno de los tres ECAs identificados, a doble-ciego de 12 semanas de seguimiento, evaluó la eficacia de un gel vaginal de estriol al $0,005 \%$ frente a placebo, en 167 mujeres en la etapa de la postmenopausia con síntomas y signos de atrofia vaginal $^{301}$. Las variables de medida de la atrofia vaginal fueron: la maduración del epitelio vaginal (variable principal), la disminución media del $\mathrm{pH}$ vaginal, la sequedad vaginal y el Global Symptom Score, además de otros signos de la atrofia vaginal. El ECA presentó limitaciones relacionadas principalmente con su diseño, que reducen su calidad y la aplicabilidad de sus resultados y conclusiones.

Este estudio mostró que el estriol en gel vaginal $(0,005 \%)$ fue significativamente superior a placebo, para mejorar la maduración del epitelio vaginal (variable principal) tras 12 semanas de tratamiento: $26,9 \pm 23.33$ vs. $3,2 \pm 16,48(\mathrm{p}<0,001)$, así como la sequedad vaginal $(\mathrm{p}=0,001)$, el Global Symptom Score $(\mathrm{p}=0,018)$ y reducir el $\mathrm{pH}$ vaginal $(-1,4 \pm 1,4$ vs. $0,3 \pm 1,2 ; \mathrm{p}<0,001) \quad(1 \mathrm{ECA}, 167$ mujeres, 12 semamas) ${ }^{301}$.

\subsection{Anillo de estrógenos frente a placebo}

La revisión sistemática ${ }^{295}$ evaluó los efectos del anillo de estradiol frente a placebo sobre los síntomas vaginales en mujeres durante la etapa de la postmenopausia.

El anillo de estradiol mostró diferencias significativas en relación a la dispareunia en comparación con placebo (1 ECA, 64 mujeres; OR 12,67; IC $95 \% 3,23$ a 49,67) ${ }^{295}$.

\section{Calidad muy baja}

\section{Calidad moderada}




\subsection{Estrógenos transdérmicos frente a placebo}

Un ECA evaluó los efectos de los estrógenos transdérmicos frente a placebo ${ }^{300}$ para el alivio de los síntomas vulvovaginales en mujeres durante la etapa de la postmenopausia.

Los estrógenos transdérmicos constataron un porcentaje Calidad baja significativamente superior de mujeres con mejorías en los síntomas de atrofia vaginal comparado con placebo ${ }^{300}$.

\subsection{Estrógenos transdérmicos a diferentes dosis frente a placebo}

Dos ECAs doble-ciego, también de 12 semanas de seguimiento publicados de forma conjunta ${ }^{95}$, evaluaron la eficacia de diferentes dosis de estradiol transdérmico (gel) en mujeres en la etapa de la postmenopausia, para determinar la dosis más eficaz y segura en el tratamiento de los síntomas vasomotores y la atrofia vulvovaginal (citología). El primer estudio en fase $3(\mathrm{n}=221)$, evaluó el gel de estradiol $(0,06 \%)$ en dosis de $1,5 \mathrm{mg}$ $(2,5 \mathrm{~g}$ de gel) y $0,75 \mathrm{mg}(1,25 \mathrm{~g}$ de gel $)$; y el segundo en fase $4(\mathrm{n}=351)$, el gel de estradiol $(0,03 \%)$ en dosis de $0,375 \mathrm{mg}(1,25 \mathrm{~g}$ de gel) y $0,27 \mathrm{mg}(0,9 \mathrm{~g}$ de gel $)$. En ambos estudios, la medida de la atrofia vulvovaginal se consideró una variable secundaria, expresada como la diferencia en el Índice de Maduración Vaginal (IVM) y/o en el Valor de Maduración (VM) desde el inicio hasta el final del tratamiento. El cálculo de ambos parámetros se realizó a partir de la citología vaginal, en función de los porcentajes de células parabasales, intermedias y superficiales. El estudio presentó limitaciones metodológicas, que dificultan el establecimiento de conclusiones definitivas y reducen la aplicabilidad de sus resultados. Los resultados se presentaron de forma confusa y no se correspondían exactamente con las variables de medida planteadas. No se presentaron resultados numéricos, ni se dispone de datos que comparen las diferentes dosis de estradiol y sólo se especificó la significación estadística de la diferencia en el IVM o del VM (según la dosis) frente a placebo. Sólo se presentaron datos numéricos relativos al VM para la dosis de $0,75 \mathrm{mg}$, pero no se presentaron resultados globales sino en función de los valores basales del VM (bajos moderados, altos).

En mujeres en la etapa de la postmenopausia, el estradiol Calidad transdérmico a dosis de $1,5 \mathrm{mg}$ y $0,75 \mathrm{mg}$ (gel $0,06 \%$ ) y de $0,3 \mathrm{mg}$ (gel muy baja $0,03 \%)$ fue significativamente más eficaz que placebo $(\mathrm{p}<0,001)$ para mejorar la atrofia vaginal (Índice de Maduración vaginal y/o Valor de Maduración), tras 12 semanas de tratamiento. La atrofia vaginal no mejoró con la dosis de $0,27 \mathrm{mg}(0,03 \%)$ (2 ECA en fase 3 y 4 , 221 mujeres en fase 3 y 351 en fase 4,12 semanas $)^{95}$.

\subsection{Anillo de estrógenos frente a crema de estrógenos}

La revisión sistemática ${ }^{295}$ evaluó los efectos del anillo de estradiol frente a la crema de estrógenos sobre los síntomas vaginales en mujeres durante la etapa de la postmenopausia.

El anillo de estrógenos en comparación con la crema de Calidad estrógenos mostró una mejoría significativa en relación al prurito moderada (2 ECA, 341 mujeres; OR 2,71; IC 95 \% 1,66 a 4,43) ${ }^{295}$. 
El anillo en comparación con la crema mostró un menor riesgo de Calidad sangrado (dos ECA, 274 mujeres; OR 0,29; IC $95 \%$ 0,11 a 0,78) 295.

\subsection{Anillo de estrógenos frente a comprimidos vaginales de estrógenos}

La revisión sistemática ${ }^{295}$ evaluó los efectos del anillo de estradiol frente a los comprimidos de estrógenos sobre los síntomas vaginales en mujeres durante la etapa de la postmenopausia.

El anillo de estrógenos en comparación con los comprimidos Calidad vaginales mostró una mejoría en la sequedad vaginal (2 ECA, 397 moderada mujeres; OR 0,40; IC 95 \% 0,24 a 0,64), dispareunia (3 ECA, 567 mujeres; OR 0,53; IC $95 \% 0,36$ a 0,78) y polaquiuria (2 ECA, 421 mujeres; OR 0,63; IC $95 \% 0,41$ a 0,95 ) a favor de los comprimidos vaginales ${ }^{295}$.

\subsection{Balance beneficios/riesgos}

Ver apartado 5.2.1.1.5 correspondiente al capítulo 5.2.1 sobre Tratamiento Hormonal.

\section{Resumen de la evidencia - estrógenos}

\begin{tabular}{|c|l|}
\hline $\begin{array}{c}\text { Calidad } \\
\text { muy baja }\end{array}$ & $\begin{array}{l}\text { Las preparaciones estrogénicas de aplicación local para mujeres con } \\
\text { síntomas de atrofia vaginal se han mostrado efectivas frente a } \\
\text { placebo para la mejoría de la sequedad, dolor, irritabilidad y } \\
\text { dispareunia vaginales }\end{array}$ \\
\hline $\begin{array}{c}\text { Calidad,299,301. } \\
\text { moderada }\end{array}$ & $\begin{array}{l}\text { Dentro de las distintas presentaciones de las preparaciones } \\
\text { estrogénicas, los óvulos de estrógenos se han mostrado más efectivos } \\
\text { que los estrógenos en crema para la mejora de la sequedad, dolor e } \\
\text { irritación vaginales en mujeres en la etapa de la postmenopausia } \\
\text { Los, óvulos de estrógenos, en las dosis habituales, no han mostrado un } \\
\text { incremento del riesgo de proliferación o hiperplasia endometrial frente } \\
\text { a placebo, aunque han mostrado mayor riesgo de sangrado vaginal que } \\
\text { el placebo }{ }^{297,299} .\end{array}$ \\
\hline $\begin{array}{c}\text { Calidad } \\
\text { moderada }\end{array}$ & $\begin{array}{l}\text { El anillo de estradiol se ha mostrado más efectivo para la mejora del } \\
\text { prúrito y la disminución del sangrado vaginal frente a la crema de } \\
\text { estrógenos y para mejorar la sequedad vaginal, la dispareunia y la } \\
\text { polaquiuria frente a los óvulos de estrógenos }\end{array}$ \\
\hline $\begin{array}{c}\text { Calidad } \\
\text { muy baja }\end{array}$ & $\begin{array}{l}\text { Los estrógenos orales } \\
\text { eficaces frente a placebo para mejorar la atrofia vaginal y la } \\
\text { sequedad vaginal en mujeres en la etapa de la postmenopausia. }\end{array}$ \\
\hline MODERADA & $\begin{array}{l}\text { En cuanto a la seguridad, la terapia hormonal combinada continua } \\
\text { aumenta significativamente el riesgo de tromboembolismo venoso, }\end{array}$ \\
\hline
\end{tabular}




\begin{tabular}{|l|l|}
\hline eventos cardiacos (infarto de miocardio o muerte cardiaca) e \\
incontinencia urinaria después de un año de uso ${ }^{81,82}$. Con este \\
tratamiento, el riesgo de ictus aumenta a los tres años y el de cáncer \\
de mama, trastornos de la vesícula biliar y mortalidad por cáncer de \\
pulmón a los 3-5,6 años. En mujeres mayores de 65 años, aumenta la \\
incidencia de demencia tras 4 años de uso \\
El. \\
$\begin{array}{l}\text { El uso a largo plazo de estrógenos (sin gestágenos) en mujeres durante } \\
\text { la etapa de la peri y postmenopausia aumenta significativamente el } \\
\text { riesgo de sufrir tromboembolismo venoso, ictus, trastornos de la } \\
\text { vesícula biliar e incontinencia urinaria (este último en mujeres } \\
\text { histerectomizadas y en mujeres con enfermedad coronaria arterial no } \\
\text { histerectomizadas), pero estudios recientes no han encontrado } \\
\text { asociación entre el uso de estrógenos (sin gestágenos) y el riesgo de } \\
\text { cáncer de mama }{ }^{81,82} \text {. No hay evidencias suficientes de que la terapia } \\
\text { hormonal tenga un impacto clínicamente significativo en la incidencia } \\
\text { de cáncer colorrectal }{ }^{82} \text {. La terapia hormonal con estradiol transdérmico } \\
\text { no modifica significativamente la incontinencia urinaria }{ }^{81} .\end{array}$ \\
\hline
\end{tabular}

\section{Recomendaciones}

\begin{tabular}{|c|l|}
\hline \multirow{2}{*}{ FUERTE } & $\begin{array}{l}\text { En mujeres en la etapa de postmenopausia con síntomas vaginales } \\
\text { moderados o intensos, se recomienda ofrecer la posibilidad de } \\
\text { realizar tratamiento con estrógenos vaginales en forma de crema, } \\
\text { óvulos o anillo vaginal, informando con claridad sobre su efectividad } \\
\text { para reducir los síntomas y sobre todos los riesgos asociados, } \\
\text { facilitando que sea la mujer quien decida si iniciar el tratamiento con } \\
\text { esta información. }\end{array}$ \\
\hline BPC & $\begin{array}{l}\text { En mujeres que conservan el útero no se requiere el uso de } \\
\text { gestágenos para evitar la hiperplasia endometrial si se emplean } \\
\text { estrógenos locales a dosis habituales. }\end{array}$ \\
\hline
\end{tabular}

\subsubsection{Gestágenos}

No se ha identificado ningún ECA que evalúe el efecto de los gestágenos sobre la atrofia vaginal. 


\subsubsection{Tibolona}

\subsection{Mujeres sanas en la etapa de la postmenopausia}

Se han identificado dos ECA que evalúan el tratamiento con tibolona para la atrofia vaginal. El ECA de Hammar et al., de $2007^{151}$ incluyó un total de 572 mujeres sanas en la etapa de la postmenopausia con sintomatología vaginal y comparó el tratamiento con tibolona oral frente al tratamiento con 17beta-estradiol más acetato de noretisterona durante 48 semanas. El ECA de Swanson et al., de 2006, con importantes limitaciones metodológicas ${ }^{153}$, incluyó un total de 396 mujeres sanas en la etapa de la postmenopausia con síntomas vasomotores intensos y atrofia genital y comparó el tratamiento con tibolona a dosis de $1,25 \mathrm{mg}$ o de $2,5 \mathrm{mg}$ frente a placebo durante 12 semanas. Además, dos estudios ${ }^{149,155}$ (una RS y un documento de síntesis de evidencia) evaluaron los efectos adversos a largo plazo derivados del empleo de tibolona en mujeres durante la etapa de la postmenopausia.

Además se identificó otro ECA que evaluó el efecto de la tibolona frente a placebo en mujeres durante la etapa de la postmenopausia para prevenir la fractura vertebral ${ }^{303} \mathrm{y}$ un estudio de cohortes $^{304}$ que investigó los efectos de tipos específicos de terapia hormonal sustitutiva sobre la incidencia de cáncer de mama.

El tratamiento con tibolona oral frente al tratamiento con 17beta- Calidad estradiol mostró una reducción significativa de la atrofia vaginal (medida moderada con índices de citología vaginal) en un grado similar en comparación a la situación basal, sin diferencias entre ellos ${ }^{151}$.

En comparación con el placebo, la tibolona a dosis de $1,25 \mathrm{mg}$ o de Calidad baja 2,5 mg aumentó de forma significativa el valor de maduración vaginal desde el inicio del estudio ${ }^{153}$.

El tratamiento con tibolona oral frente a placebo redujo Calidad significativamente el riesgo de cáncer de mama invasivo (25 eventos; moderada relative hazard 0,32 ; IC $95 \% 0,13$ a 0,80) y mostró una reducción en las fracturas no vertebrales (288 eventos; relative hazard 0,74; IC $95 \% 0,58$ a $0,93$ ) y vertebrales (196 eventos; relative hazard 0,55 ; IC $95 \% 0,41$ a 0,74$)^{303}$.

Los diferentes ECA no muestran a corto plazo efectos adversos Calidad importantes derivados del empleo de tibolona. Se ha descrito un aumento moderada del riesgo de sangrados irregulares y una disminución de las lipoproteínas de alta densidad y algunos estudios muestran efectos adversos androgénicos (hirsutismo, piel grasa), pero sin mostrar resultados consistentes $^{149,155}$. El estudio que comparó el efecto de la tibolona frente a placebo en mujeres durante la etapa de la postmenopausia para prevenir la fractura vertebral se suspendió prematuramente debido a un aumento del riesgo de accidente cerebrovascular (41 eventos; relative hazard 2,19; IC $95 \% 1,14$ a 4,23) $)^{303}$. Además, el estudio de cohortes mostró un incremento significativo de la incidencia de cáncer de mama en el grupo de mujeres que recibían tibolona (RR 1,45; IC $95 \%$ 1,25-1,68) ${ }^{304}$. 


\subsection{Mujeres en la etapa de la postmenopausia con cáncer de mama}

Un ECA evaluó el efecto de la tibolona en mujeres con cáncer de mama ${ }^{305}$ para tratar los síntomas vasomotores.

Este estudio se suspendió prematuramente debido a un aumento del Calidad riesgo de recurrencia de cáncer de mama (402 eventos; hazard ratio 1,40; moderada IC $95 \% 1,14$ a 1,70$)^{305}$.

\section{Resumen de la evidencia - tibolona}

\begin{tabular}{|c|l|}
\hline $\begin{array}{c}\text { Calidad } \\
\text { baja }\end{array}$ & $\begin{array}{l}\text { En comparación con placebo, la tibolona es más efectiva para } \\
\text { aumentar la maduración vaginal }{ }^{153} \text {. }\end{array}$ \\
\hline $\begin{array}{c}\text { Calidad } \\
\text { moderada }\end{array}$ & $\begin{array}{l}\text { La tibolona oral se ha mostrado tan efectiva como los estrógenos } \\
\text { orales para mejorar los síntomas de la atrofia vaginal }^{151} \text {. }\end{array}$ \\
\hline $\begin{array}{c}\text { Calidad } \\
\text { moderada }\end{array}$ & $\begin{array}{l}\text { La tibolona puede aumentar el riesgo de accidente cerebrovascular } \\
\text { y parece disminuir las fracturas no vertebrales y vertebrales }\end{array}{ }^{303}$. Los \\
resultados sobre la relación de la tibolona y el riesgo de cáncer de \\
mama no son concluyentes
\end{tabular}

\section{Recomendaciones}

\begin{tabular}{|l|l|}
\hline \multirow{2}{*}{ DÉBIL } & $\begin{array}{l}\text { En mujeres en la etapa de la postmenopausia, con síntomas } \\
\text { vaginales moderados o intensos y las que el tratamiento hormonal } \\
\text { con estrógenos no haya sido efectivo o no se tolere, se sugiere } \\
\text { valorar el tratamiento con tibolona para los síntomas vaginales, } \\
\text { teniendo en cuenta el riesgo/beneficio de esta intervención. }{ }^{\text {d }}\end{array}$ \\
\hline
\end{tabular}

\subsubsection{Fitoterapia}

Pocos estudios han evaluado el efecto de la fitoterapia sobre los síntomas vaginales como objetivo primario. Diversos estudios han evaluado los síntomas relacionados con la menopausia con escalas que pueden incluir o no síntomas relacionados con atrofia vaginal aunque en muy pocos estudios el resultado de estas escalas se presenta desagregado por síntomas.

\footnotetext{
d Ver Recomendación de BPC referida a Tibolona en "Tratamiento de los síntomas vasomoteres".
} 


\subsubsection{Fitoestrógenos}

Se han identificado varias RS que evaluaron el tratamiento con fitoestrógenos para los síntomas vasomotores y algunos de los ECA incluidos valoraron también síntomas vaginales $^{120,165,195,306,307}$. Se localizaron adicionalmente estudios posteriores o no incluidos en las RS que evaluaron los síntomas vaginales.

\subsection{Isoflavonas de trébol rojo (Trifolium pratense; red clover)}

Un ECA cruzado, doble ciego, que incluyó a 60 mujeres con síntomas relacionados con la menopausia aleatorizadas a recibir trébol rojo o placebo en dos periodos de tres meses de duración, mostró una mejora estadísticamente significativa en el índice de maduración vaginal (una medida objetiva de la mejora de la atrofia) respecto al placebo ${ }^{308}$.

\subsection{Isoflavonas de soja: extractos, concentrados, suplementos dietéticos}

Un ECA reciente (236 mujeres con sofocos en las que se evaluaron los síntomas vaginales) mostró que el tratamiento con extractos de soja (genisteína) no produjo diferencias significativas en el índice de maduración vaginal en comparación con el placebo ${ }^{222}$.

Un estudio, no publicado, no mostró diferencias frente a placebo respecto al índice de maduración vaginal ${ }^{210}$. Otro estudio comparó los extractos estandarizados de soja con estrógenos y mostró una mejora significativa en el índice de maduración vaginal en el grupo de tratamiento con estrógenos pero no en el de tratamiento con soja ${ }^{219}$.

Un ECA cruzado (36 mujeres) evaluó los efectos de una dieta rica en soja o una dieta libre de productos de soja durante 12 semanas. La sequedad vaginal se incrementó significativamente tras 12 semanas de dieta libre de soja. Tras 12 semanas de una dieta rica en soja, la sequedad vaginal disminuyó, aunque no de forma significativa ${ }^{309}$.

Otros dos ECA que evaluaron los suplementos dietéticos de soja no mostraron diferencias respecto a placebo en el índice de maduración vaginal $^{232,233}$. Un tercer estudio mostró una mejora significativa respecto al inicio en la valoración subjetiva de sequedad vaginal en el grupo intervención pero no en el grupo placebo, aunque no se compararon ambos grupos ${ }^{234}$.

Un ECA (60 mujeres en etapa postmenopáusica 40-60 años), evaluó la eficacia de los suplementos dietéticos de soja ( $90 \mathrm{mg} / \mathrm{d}$ de isoflavonas) frente a placebo y frente a terapia hormonal (estradiol $1 \mathrm{mg} / \mathrm{d}$ + noretisterona $0,5 \mathrm{mg} / \mathrm{d}$ ), para reducir los síntomas urogenitales, psicológicos y somáticos asociados a la menopausia, tras 16 semanas de tratamiento ${ }^{310}$.

Las isoflavonas de soja no modificaron significativamente la maduración vaginal, mostrando resultados más desfavorables que la terapia hormonal (DM -18,1) y que el placebo (DM -3,3). Redujeron

Calidad baja

\section{Calidad \\ muy baja}


significativamente la sequedad vaginal frente a placebo (Menopause

Rating Scale $)(\mathrm{p}=0,02)$, pero no frente a la terapia hormonal $(\mathrm{p}=0,28)$.

\subsubsection{Cimifuga Racemosa (Black cohosh)}

Se han identificado dos revisiones sistemáticas sobre Cimifuga Racemosa que analizaron su eficacia para aliviar los síntomas asociados a la menopausia, incluyendo los síntomas vaginales ${ }^{311,312}$.

Una revisión sistemática/metanálisis ${ }^{311}$, analizó 16 ECA (duración media 23 semanas), en un total de 2.027 mujeres en etapas peri y postmenopáusica, para evaluar la eficacia y/o seguridad de Cimifuga Racemosa en monoterapia (dosis media $40 \mathrm{mg} /$ día) en el tratamiento de la sintomatología asociada a la menopausia. Los síntomas vulvovaginales se analizaron en 4 ECA frente a placebo, que midieron la frecuencia o intensidad de la atrofia vaginal (sequedad, hemorragia y/o prurito). En la RS no se presentan resultados de dichos ECA al considerarlos insuficientes para realizar un análisis adecuado y se concluyó que la evidencia disponible resultaba insuficiente y/o inadecuada para justificar el uso de Cimicífuga racemosa en los síntomas vaginales asociados a la menopausia.

Otra revisión sistemática ${ }^{312}$ analizó 12 ECA (12 semanas-12 meses), en un total de 1.573 mujeres, para evaluar la eficacia de Cimicífuga racemosa, Hypericum perforatum y Agnus castus, en monoterapia o combinados, en el tratamiento de los síntomas vasomotores, vaginales y psicosociales asociados a la menopausia. No se realizó metanálisis de los ECA al considerar los datos insuficientes y heterogéneos. Los datos relativos al efecto de Cimifuga Racemosa en los síntomas vaginales (atrofia vaginal) proceden de cuatro ECA frente a placebo, dos de ellos en combinación con Hypericum perforatum.

Un ECA (351 mujeres) no mostró diferencias significativas en el análisis citológico (índice de maduración vaginal) entre las mujeres que recibieron Cimifuga Racemosa o placebo a los 12 meses ni en el índice de sequedad vaginal ${ }^{313}$.

Dos ECA (190 mujeres) mostraron una reducción significativa de los síntomas relacionados con la atrofia vaginal de la MRS (Menopause Rating Scale) en las mujeres tratadas con Cimifuga Racemosa, en comparación con placebo ${ }^{314,315}$.

\subsubsection{Pueraria mirifica}

Un ECA (86 mujeres) mostró una sequedad vaginal significativamente superior en el grupo placebo en comparación con el grupo de Calidad baja tratamiento con Pueraria mirifica $(1,45 \pm 1,19$ a $1,08 \pm 1,07)$. No se mostraron diferencias para otros síntomas urogenitales ${ }^{309}$. 


\section{Resumen de la evidencia - fitoterapia}

\begin{tabular}{|c|c|}
\hline $\begin{array}{c}\text { Calidad } \\
\text { BAJA o } \\
\text { MUY BAJA }\end{array}$ & $\begin{array}{l}\text { La soja (en forma de extractos estandarizados o como suplemento } \\
\text { dietético) }{ }^{210,219,222,232-234,309,310} \text {, el trébol rojo }{ }^{308} \text {, la Cimifuga Racemosa }{ }^{311-315} \text { y } \\
\text { la Pueraria mirifica } \text { R }^{309} \text { no parecen mejorar los síntomas vaginales ni } \\
\text { tener efectos sobre la maduración del epitelio vaginal en mujeres en } \\
\text { etapa postmenopáusica. }\end{array}$ \\
\hline
\end{tabular}

\section{Recomendaciones}

DÉBIL

Se sugiere no utilizar el tratamiento con fitoestrógenos, Cimifuga Racemosa o Pueraria mirifica para tratar los síntomas vaginales en las mujeres durante la peri y postmenopausia. 


\subsubsection{Moduladores selectivos de los receptores estrogénicos (SERM)}

Los moduladores selectivos de los receptores estrogénicos (SERM) son un grupo heterogéneo de fármacos que tienen un mecanismo de acción complejo que implica una acción agonista o antagonista de los receptores estrogénicos en diferentes tejidos. En los estudios identificados se ha evaluado la eficacia del ospemifeno (en fase de investigación ya que aunque está aprobado por la Food and Drug Administration, no está aprobado por la European Medicines Agency), el bazedoxifeno y el raloxifeno, para tratar los síntomas relacionados con la atrofia vaginal.

\subsubsection{Ospemifeno}

Con el objetivo de evaluar la seguridad del ospemifeno a largo plazo, se ha identificado un ECA (826 mujeres) que aleatorizó las mujeres en tres grupos de estudio según recibieran $60 \mathrm{mg}$ de ospemifeno $(\mathrm{N}=276), 30 \mathrm{mg}(\mathrm{N}=282)$ o placebo $(\mathrm{N}=286)$ durante 12 semanas $^{316}$.

Adicionalmente se ha identificado un estudio posterior que realizó un seguimiento adicional de 40 semanas (180 mujeres) ${ }^{317}$ de este ECA inicial ${ }^{316}$, manteniendo los mismos grupos de tratamiento (ospemifeno a dosis de $30 \mathrm{mg} /$ día, ospemifeno a $60 \mathrm{mg} /$ día y placebo). Se cuantificaron los efectos adversos, los cambios en la histología y el grosor endometrial, se realizaron tests cervicales, examen ginecológico, mamografía y palpación de mamas, y pruebas de laboratorio de seguridad clínica. Los resultados muestran los valores iniciales y a las 52 semanas de tratamiento, aunque sin citar la significación estadística (p, IC) ni realizar comparaciones entre los distintos brazos del tratamiento.

En el ECA inicial de 12 semanas, el análisis citológico mostró una Calidad mejoría significativa a las 4 y 12 semanas para los grupos de tratamiento moderada activo frente a placebo, así como para la valoración subjetiva de sequedad vaginal $^{316}$. En cuanto a su seguridad, en el estudio de seguimiento de 40 semanas adicionales, realizado en un total de 180 mujeres, no se observaron efectos adversos clínicamente significativos en ningún grupo de tratamiento, siendo los sofocos el efecto adverso más frecuente ${ }^{317}$.

\subsubsection{Bazedoxifeno}

Se han localizado dos publicaciones de un mismo ECA que ha evaluado el bazedoxifeno en mujeres en etapa postmenopáusica con síntomas de atrofia vaginal. El ECA aleatorizó 664 mujeres en tres brazos de tratamiento con bazedoxifeno $20 \mathrm{mg}$ (dos combinados con estrógenos y uno en monoterapia) y un cuarto brazo de placebo durante un seguimiento total de 12 semanas.

Este ECA mostró que sólo el tratamiento combinado con estrógenos Calidad baja proporcionaba resultados favorables para la sequedad vaginal y otros síntomas relacionados (picor y dolor), respecto a placebo ${ }^{318,319}$. 


\subsubsection{Raloxifeno}

Se ha identificado un ECA a doble ciego que comparaba los efectos urogenitales del raloxifeno, tamoxifeno, estrógenos equinos conjugados y placebo en mujeres sanas durante la etapa de la postmenopausia ${ }^{320}$. Además, se identificaron dos ECA (278 mujeres) que evaluaban el uso de raloxifeno para paliar los síntomas (sequedad, picor, palidez y adelgazamiento de la mucosa, petequias o atrofia labial) que sufrían las mujeres que recibían cremas vaginales con o sin contenido hormonal o un dispositivo liberador tópico de estrógenos. En ambos estudios se aleatorizaban las mujeres a recibir raloxifeno $60 \mathrm{mg}$ o placebo ${ }^{321,322}$. Otros dos estudios ${ }^{323,324}$ analizaron los efectos secundarios asociados al empleo de raloxifeno para tratar la osteoporosis y el perfil lipídico en mujeres en la etapa de la postmenopausia.

El raloxifeno no mostró una mejora de la sintomatología vaginal Calidad baja frente a tamoxifeno, estrógenos equinos conjugados y placebo en mujeres en etapa postmenopáusica sanas. Este beneficio sólo se observó con el empleo de estrógenos ${ }^{320}$.

En ninguno de los dos estudios el raloxifeno modificó el efecto de los estrógenos tópicos o de los lubricantes hormonales ${ }^{321,322}$.

El raloxifeno mostró un aumento del riesgo de tromboembolismo a los cuatro años de seguimiento (RR 2,13; IC 95 \% 1,21 a 3,75). También se han descrito otros efectos adversos con el tratamiento con raloxifeno, como síntomas vasomotores e hiperplasia endometrial, aunque a su vez también ha demostrado reducción de fractura vertebral y de cáncer de mama estrogenodependiente ${ }^{323,324}$.

Resumen de la evidencia - Moduladores selectivos de los receptores estrogénicos (SERM)

\begin{tabular}{|c|l|}
\hline $\begin{array}{c}\text { Calidad } \\
\text { MODERADA }\end{array}$ & $\begin{array}{l}\text { El ospemifeno mejora la sequedad vaginal en mujeres durante la } \\
\text { etapa postmenopáusica frente a placebo y no ha mostrado efectos } \\
\text { adversos clínicamente significativos, siendo los sofocos el síntoma } \\
\text { más frecuente asociado a su empleo. Sin embargo, la comercialización } \\
\text { de este fármaco no está aprobada por la Agencia Española del } \\
\text { Medicamento. }\end{array}$ \\
\hline $\begin{array}{c}\text { Calidad } \\
\text { BAJA }\end{array}$ & $\begin{array}{l}\text { El bazedoxifeno sólo ha mostrado eficacia (disminución de la } \\
\text { sequedad vaginal y otros síntomas relacionados como picor y dolor) } \\
\text { para el tratamiento de los síntomas vaginales de mujeres en etapa } \\
\text { postmenopáusica en combinación con un tratamiento con estrógenos } \\
\text { frente a placebo }\end{array}$ \\
\hline $\begin{array}{c}\text { Calis,319. } \\
\text { BAJA }\end{array}$ & $\begin{array}{l}\text { El raloxifeno no ha mostrado una mejora de la sintomatología vaginal } \\
\text { frente a placebo, tamoxifeno y estrógenos equinos conjugados en } \\
\text { mujeres en etapa postmenopáusica sanas y no parece modificar el }\end{array}$ \\
\hline
\end{tabular}




\begin{tabular}{|l|l|}
\hline $\begin{array}{l}\text { efecto de los estrógenos tópicos o de los lubricantes no } \\
\text { hormonales }^{321,322,324} \text {. El raloxifeno presenta un aumento de riesgo de } \\
\text { tromboembolia y puede presentar otros efectos adversos como } \\
\text { síntomas vasomotores e hiperplasia endometrial }^{323,324} .\end{array}$ \\
\hline
\end{tabular}

\section{Recomendaciones}

\begin{tabular}{|c|l|}
\hline \multirow{2}{*}{ DÉBIL } & $\begin{array}{l}\text { Se sugiere no utilizar el tratamiento con moduladores selectivos de } \\
\text { los receptores estrogénicos (ospemifeno, bafedoxifeno y raloxifeno) } \\
\text { para mejorar los síntomas vaginales en mujeres durante las etapas } \\
\text { peri y postmenopáusica. }\end{array}$ \\
\hline
\end{tabular}




\subsubsection{Lubricantes e hidratantes}

No se dispone de ECAs que comparen la efectividad de los lubricantes con Calidad baja el placebo para los síntomas vaginales. Se han identificado dos ECA que comparan las cremas hidratantes vaginales con estrógenos en crema y que han mostrado una eficacia similar en la dispareunia, aunque los estrógenos fueron más efectivos para la sequedad vaginal que los hidratantes ${ }^{325,326}$.

\section{Resumen de la evidencia - lubricantes e hidratantes}

Calidad

Los lubricantes vaginales han mostrado una eficacia similar que los

BAJA estrógenos en la dispareunia, pero estos últimos son más efectivos para tratar la sequedad vaginal ${ }^{325,326}$.

\section{Recomendaciones}

DÉBIL

En mujeres con síntomas de dispareunia durante la peri y postmenopausia se sugiere valorar el uso de lubricantes e hidratantes vaginales. 


\section{Estrategias diagnósticas y terapéuticas}

Las estrategias terapéuticas presentadas en este capítulo están basadas en las síntesis de evidencia y las recomendaciones proporcionadas por la guía y se han realizado con el consenso de autoras y autores y las personas que revisaron la guía.

\section{Algoritmo 1. Mujeres con síntomas vasomotores}

1. Evaluar la presencia de sofocos, así como su intensidad, frecuencia y repercusión sobre la calidad de vida. Preguntar por la presencia de sudoraciones nocturnas y por posibles alteraciones del sueño. Evaluar posible presencia de síntomas vaginales.

2. Si los síntomas son percibidos como importantes, valorar con la mujer la posibilidad de iniciar tratamiento. Explicar la naturaleza autolimitada y la benignidad de los síntomas así como los riesgos y beneficios derivados del tratamiento. Recomendar medidas para no exacerbar la sintomatología (procurar un ambiente fresco, disminuir el consumo de cafeína, bebidas calientes, alcohol y comidas picantes, así como controlar el peso, realizar ejercicio físico y suprimir el tabaco) y recomendar tratamiento hormonal (estrógenos, con o sin gestágenos o tibolona).

3. Si la mujer decide la opción del tratamiento y no presenta ninguna contraindicación para el mismo (antecedentes de cáncer de mama, enfermedad coronaria, tromboembolismo e ictus o bien con alto riesgo de padecer estas enfermedades, así como hepatopatía aguda o crónica, enfermedad pancreática y de vesícula biliar), iniciar el tratamiento con la menor dosis eficaz y valorar cada seis semanas si es preciso un aumento de la dosis hasta la remisión de la sintomatología. Las mujeres no histerectomizadas deben recibir un gestágeno además del estrógeno.

Una vez alcanzado el efecto deseado, mantener el tratamiento por periodos entre seis meses y un año. A partir de entonces puede intentarse un proceso inverso de disminución gradual de la dosis. En caso de observarse la reaparición de la sintomatología, puede volverse a la dosis mínima eficaz.

4. Si la mujer no acepta el tratamiento hormonal, valorar el tratamiento con fitoestrógenos o con antidepresivos (paroxetina o desvenlafaxina) o la gabapentina. Estos fármacos no tienen indicación registrada en nuestro país para el tratamiento de los síntomas vasomotores.

5. Si la sintomatología es leve, comentar la benignidad y la naturaleza autolimitada de los mismos y recomendar las medidas para no exacerbar la sintomatología. 
6. Si persiste la sintomatología o la mujer desea iniciar tratamiento, se puede valorar el uso de fitoestrógenos.

7. Si persisten los síntomas o se intensifican, valorar inicio de tratamiento hormonal y proceder según punto 1.3.

\section{Notas prácticas sobre el tratamiento hormonal}

- Antes de iniciar el tratamiento hormonal se debe realizar una historia clínica.

- Valorar realizar una mamografía en mujeres en tratamiento hormonal con estrógenos y gestágenos menores de 50 años. En las mujeres de más de 50 años la periodicidad de la mamografía debería ser cada 2 años (sobre todo en las mujeres en tratamiento hormonal con estrógenos sin gestágenos).

- A los 3-6 meses de iniciado el tratamiento, valorar la respuesta clínica y posibles efectos adversos.

- En la pauta continua se consideran aceptables las hemorragias irregulares durante el primer año de tratamiento. Si éstas persisten, realizar un estudio endometrial.

- En la pauta cíclica se consideran normales aquellas hemorragias que aparecen a partir del día 10 tras iniciar el progestágeno y, cuya cantidad y duración es similar o inferior a las menstruaciones de la etapa fértil. En caso contrario, ajustar la dosis del progestágeno aumentando los días y/o la dosis diaria. Si éstas persisten, realizar un estudio endometrial.

- El seguimiento de la terapia hormonal debe ser clínico (en función de la persistencia o no de la sintomatología por la que se indica, y en función de los efectos secundarios del tratamiento, básicamente metrorragias) y no se debe basar en la evaluación de los niveles hormonales, por lo que no es preciso realizar una analítica hormonal antes de iniciar el tratamiento ni durante el curso del mismo.

- No es preciso realizar un estudio endometrial, salvo que el patrón de sangrado sea anómalo, en cuyo caso se podría utilizar la ecografía transvaginal para una primera aproximación diagnóstica, y decidir la conducta a seguir en función del grosor del endometrio.

- La administración de los estrógenos sistémicos debería limitarse a los cinco años y posteriormente, el tratamiento/indicación debería ser individualizado. 
Algoritmo 1. Mujeres con síntomas vasomotores

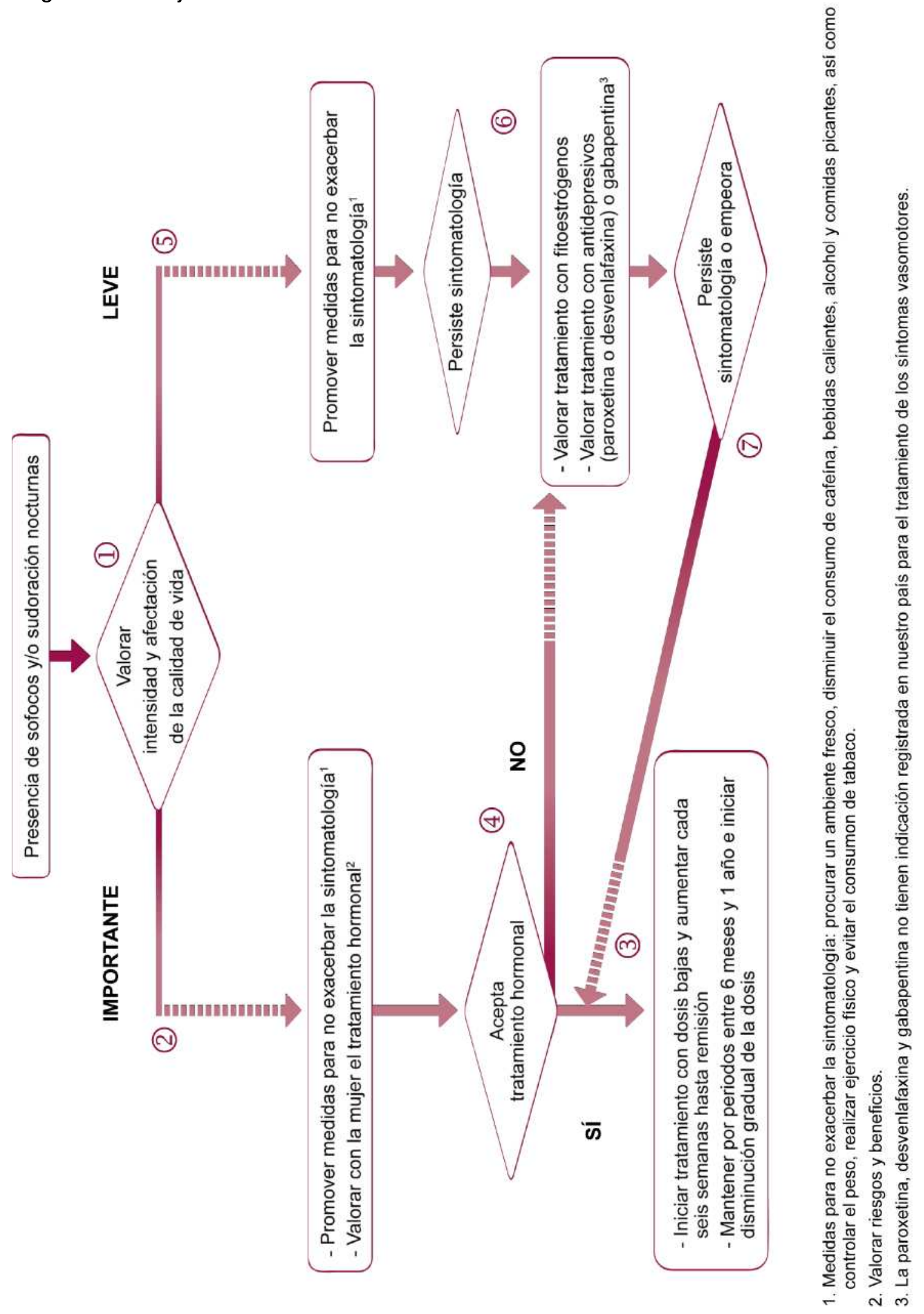




\section{Algoritmo 2. Mujeres con síntomas vaginales}

1. Valorar presencia de síntomas vaginales (sequedad, dispareunia, irritación, disuria) e intensidad.

2. Si la sintomatología es importante y no cede con lubricantes y/o hidratantes vaginales, recomendar el tratamiento con estrógenos tópicos (en crema u óvulos). Administrar diariamente durante 2 a 3 semanas y a continuación de 2 a 3 veces por semana. En las mujeres que conservan el útero no es necesario asociar gestágenos al tratamiento.

3. Revisar la sintomatología al mes de tratamiento y ajustar la dosis en caso necesario.

4. Si la sintomatología ha remitido, mantener terapia de mantenimiento durante unas semanas y suspender. Valorar el uso de lubricante o crema hidratante en caso necesario. Si aparecen de nuevo los síntomas, plantear reinicio del tratamiento. Si la sintomatología intensa no remite con estrógenos tópicos, valorar remitir a consulta de ginecología para descartar otras patologías y/o plantear tratamiento sistémico.

5. Si la sintomatología inicial es leve o la mujer no acepta el tratamiento hormonal, valorar el uso de un lubricante o crema hidratante.

6. Revisar sintomatología al mes de tratamiento y en caso de que no haya mejoría, valorar el posible tratamiento con estrógenos tópicos (valorar el tratamiento con gel, óvulos o anillo) y proceder según punto 2.2.

7. Si la sintomatología remite, mantener el tratamiento a demanda. 
Algoritmo 2. Mujer con síntomas vaginales

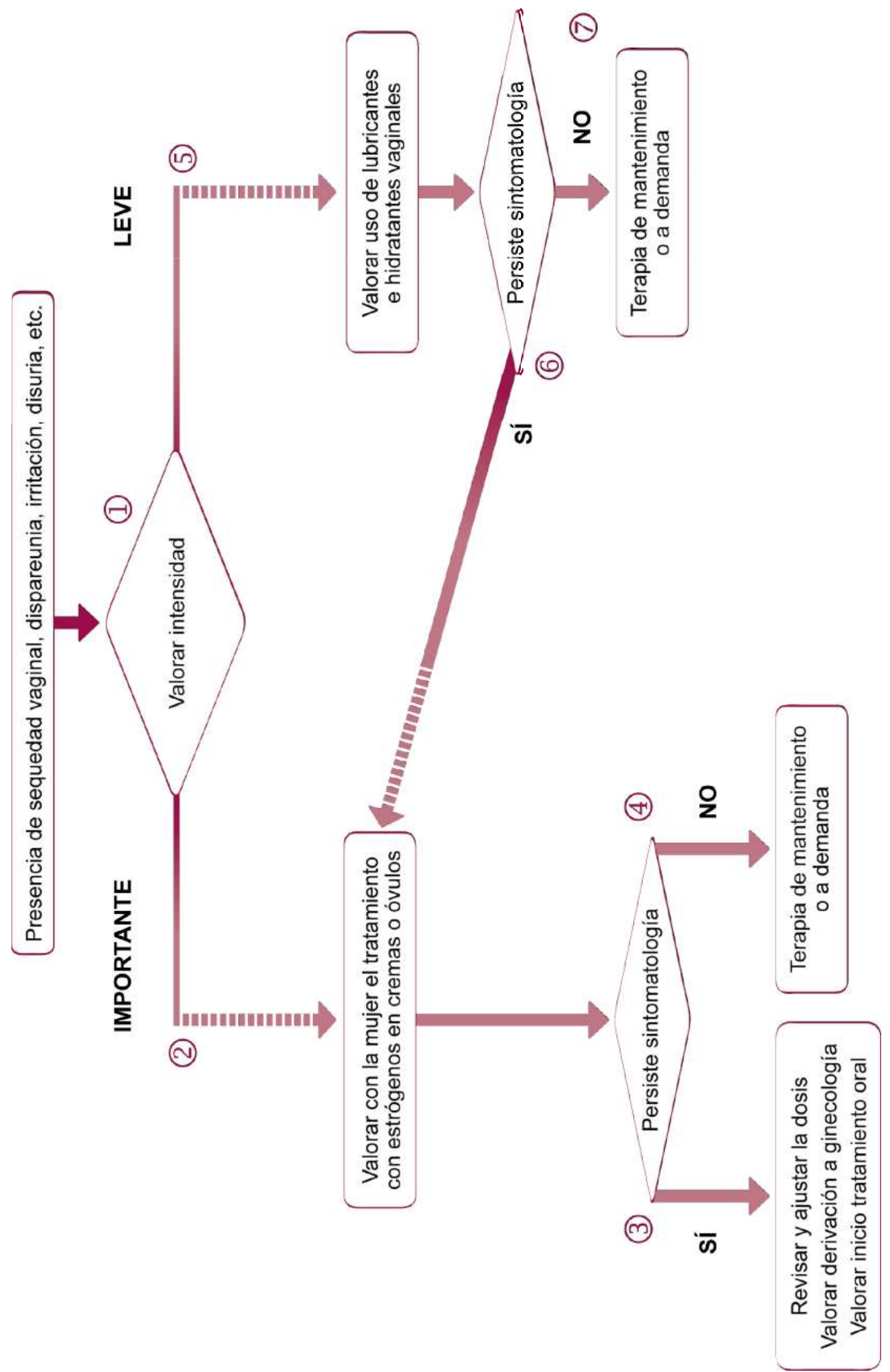





\section{Difusión e implementación}

\subsection{Formatos de la guía, difusión e implementación}

La GPC se presenta en versión completa y resumida. Además se ha elaborado una guía de información para mujeres. Todas las versiones están disponibles en formato HTLM y en formato PDF en la página web de Guía Salud (www.guiasalud.es).

Las estrategias de difusión e implementación comprenden:

1. Presentación oficial de la guía por parte de las autoridades sanitarias.

2. Envío individualizado de ejemplares a profesionales y a potenciales personas usuarias.

3. Distribución de material para mujeres.

4. Difusión de la guía en formato electrónico en las páginas web de los servicios de salud y de las sociedades implicadas en el proyecto.

5. Presentación de la guía en actividades científicas (jornadas, congresos, reuniones, etc.).

6. Publicación de la guía en revistas médicas.

\subsection{Propuesta de indicadores}

El equipo elaborador de esta GPC, junto con la ayuda de colaboradores y revisores externos, han diseñado unos indicadores con la finalidad de proveer unas herramientas que sirvan para evaluar el grado de cumplimiento de las principales recomendaciones de esta GPC. Estos indicadores se han elaborado a partir de aquellas recomendaciones con mayor nivel de evidencia y/o mayor consenso entre los autores de la guía.

\section{En las consultas sanitarias:}

- Porcentaje de consultas que disponen/ofrecen a las mujeres información resumida basada en la guía (folletos, hojas,...).

- Porcentaje de profesionales que siguen las recomendaciones de la guía en sus decisiones clínicas (adherencia):

- Porcentaje de mujeres en etapa postmenopaúsica que reciben información sobre consejos sobre estilos de vida y se recoge en la historia clínica.

- Porcentaje de mujeres con síntomas intensos que afectan su calidad de vida que reciben tratamiento hormonal o terapia no farmacológica y se recoge en la historia clínica.

- Porcentaje de mujeres en tratamiento con algún fármaco o terapia no farmacológica cuyo uso es desaconsejado en la guía. 
- Porcentaje de mujeres en tratamiento con algún fármaco o terapia no farmacológica cuyo uso es desaconsejado en la guía.

- Porcentaje de mujeres en tratamiento con terapia hormonal que han sido reevaluadas sobre la necesidad de la misma o se ha suspendido el tratamiento. 


\section{Líneas de investigación futura}

\section{Manifestaciones clínicas de la menopausia}

Estudios longitudinales sobre manifestaciones clínicas de la menopausia en mujeres de distintas etnias y grupos raciales.

Estudios observacionales que determinen el papel del ejercicio regular, la nutrición óptima, el apoyo social en la presentación de los síntomas asociados con la menopausia.

Estudios osbservacionales que determinen el papel de suprimir determinadas acciones en la presentación de síntomas vasomotores (como perder peso en mujeres con obesidad, abandonar el consumo de tabaco en mujeres fumadoras, etc.)

\section{Terapia hormonal}

ECAs que permitan determinar las dosis óptimas efectivas, la combinación de regímenes y el tiempo de tratamiento.

ECAs que examinen la seguridad endometrial del uso a corto plazo $(\leq 1$ año) de estrógenos solos a dosis bajas.

ECAs que analicen los riesgos y beneficios de los dispositivos intrauterinos que liberan gestágenos en combinación con dosis bajas de estrógenos orales para la terapia a corto y largo plazo en mujeres durante las etapas peri y postmenopáusicas.

ECAs que evalúen cómo suspender el tratamiento cuando los síntomas desaparecen, incluyendo la efectividad de reducir progresivamente la dosis y/o reemplazarla con intervenciones no farmacológicas.

ECAs con comparaciones directas de distintas formulaciones, régimenes y dosis tanto de estrógenos como de gestágenos.

Estudios que permitan determinar el papel de los gestágenos (clase de gestágeno y régimen de administración) en el cáncer de mama y otras variables de resultado.

Identificación de los mecanismos que conllevan los efectos diferenciales de la terapia hormonal en el riesgo cardiovascular en mujeres jóvenes en etapa postmenopáusica recientes en comparación con mujeres mayores en etapa postmenopáusica desde hace años.

ECAs que evalúen la eficacia de las hormonas bioidénticas.

Estudios en mujeres con características específicas que no se hayan evaluado previamente como mujeres con fallo ovárico prematuro, mujeres en tratamiento con los nuevos y futuros SERM, mujeres con IMC muy alto o muy bajo o con estilos de vida que puedan influenciar la aparición de síntomas. 


\section{Otros tratamientos}

ECA que permitan conocer la eficacia y la seguridad de los tratamientos no hormonales.

ECA que evalúen los efectos de la relajación y de las terapias cuerpo-mente en el manejo de los síntomas vasomotores. 


\section{Anexos}

\section{Anexo 1. Evaluación de la calidad de la evidencia y la graduación de las recomendaciones mediante el sistema GRADE}

Cuando se formulan recomendaciones, el grupo elaborador de la guía debe plantearse hasta qué punto se puede confiar en que llevar a cabo una recomendación conllevará más beneficios que daños. Esta decisión no es sencilla y está influida por múltiples factores que convierten esta etapa en una de las más complejas de la elaboración de una guía.

Los sistemas de formulación de recomendaciones se comenzaron a proponer hace más de dos décadas. Estos sistemas diferenciaban ya inicialmente el nivel de evidencia científica (la adecuación de los diferentes diseños de estudios para contestar a los diferentes tipos de preguntas) de la fuerza de las recomendaciones. Desde entonces los diferentes sistemas han ido evolucionando e incorporando otros aspectos más allá del diseño de los estudios que hay que tener en cuenta a la hora de formular recomendaciones.

Para clasificar la calidad de la evidencia y graduar la fuerza de las recomendaciones se han seguido las directrices del grupo de trabajo GRADE. Este grupo de trabajo intenta establecer un método de elaboración de recomendaciones explícito y transparente, de fácil manejo por los equipos elaboradores de GPC, con el objetivo de superar los inconvenientes de otros sistemas de elaboración de recomendaciones ${ }^{12}$.

Las principales etapas que conforman el sistema GRADE (Grading of Recommendations of Assessment Development and Evaluation) se describen a continuación:

\section{A) Clasificación de la importancia relativa de las variables de resultado}

En esta etapa el sistema GRADE recomienda que en la etapa inicial de la formulación de las preguntas clínicas el grupo elaborador establezca de forma explícita las variables de resultado de interés para las preguntas y clasifique su importancia relativa. Se recomienda clasificar su importancia mediante la siguiente escala de nueve puntos:

1-3: variable de resultado no importante. No se deben incluir en la tabla de evaluación de la calidad o de resultados. Estas variables de resultado no jugarán un papel importante enx ${ }^{\mathrm{o}}$ la formulación de las recomendaciones.

4-6: variable de resultado importante pero no clave para la toma de decisiones.

7 - 9: variable de resultado clave para la toma de decisiones.

La importancia relativa de las variables de resultado se establece mediante consenso. 


\section{B) Evaluación de la calidad de la evidencia científica}

La evaluación de la calidad se realiza para cada una de las variables de resultado seleccionadas. Por tanto, para una pregunta clínica habrá muy probablemente variables de resultado con diferentes calificaciones de calidad. Inicialmente se debe evaluar la evidencia científica por el diseño de los estudios y su adecuación para responder a cada tipo de pregunta de la guía. Inicialmente, se considera como "calidad alta" a los ECA y como "calidad baja" a los estudios observacionales. No obstante se sugieren una serie de aspectos que pueden disminuir la calidad de los ECA o que la pueden aumentar en el caso de los estudios observacionales. La calidad de la evidencia científica se califica como alta, moderada, baja o muy baja.

El grupo elaborador ha clasificado la calidad de la evidencia para el conjunto de variables que se han considerado para formular cada recomendación. En la clasificación de la calidad global de la evidencia, el grupo elaborador ha considerado:

- Principalmente las variables críticas para la toma de decisiones.

- Cuando éstas han mostrado un resultado inconsistente (hacia un beneficio y hacia un daño) se ha considerado la calidad global como la más baja entre las diferentes variables.

- Cuando éstas han mostrado un resultado consistente (hacia un beneficio o un daño) se ha considerado la calidad global como la más alta entre las diferentes variables.

- Cuando éstas han mostrado un resultado consistente pero el balance entre los beneficios y los perjuicios es incierto se ha considerado la calidad global como la más baja entre las diferentes variables.

Aspectos que pueden disminuir la calidad de los ECA:

- Limitaciones en el diseño o en la ejecución: como pueden ser la ausencia de ocultamiento de la secuencia de aleatorización, enmascaramiento inadecuado, pérdidas importantes, ausencia de análisis por intención de tratar, finalización del estudio antes de lo previsto por razones de beneficio.

- Resultados inconsistentes: estimaciones discrepantes del efecto de un tratamiento (heterogeneidad o variabilidad en los resultados) en los estudios disponibles sugieren diferencias reales en dichas estimaciones. Estas pueden deberse a diferencias en la población, en la intervención, en las variables de resultado o en la calidad de los estudios. Cuando exista heterogeneidad no explicada de manera razonable la calidad disminuye.

- Ausencia de evidencia científica directa: en el caso de ausencia de comparaciones directas entre dos tratamientos (comparación de cada tratamiento frente a placebo, pero no entre ellos) o la extrapolación de los resultados de un estudio con un determinado fármaco al resto de fármacos de su misma familia en ausencia de un efecto de clase demostrado, también se considera evidencia científica indirecta. Es frecuente que existan grandes diferencias entre la 
población en la que se aplicarán las recomendaciones y la incluida en los estudios evaluados. Por último, deben ser también valorados los aspectos de la potencial aplicabilidad en nuestro entorno o la validez externa de la evidencia científica disponible.

- Imprecisión: cuando los estudios disponibles incluyen relativamente pocos eventos y pocos/as pacientes y, por tanto, presentan intervalos de confianza amplios, la calidad disminuye.

- Sesgo de notificación: la calidad puede disminuir si se tiene la duda razonable sobre si la autoría ha incluido o no todos los estudios (por ejemplo, el sesgo de publicación en el contexto de una RS) o si se han incluido o no todas las variables relevantes de resultado (outcome reporting bias).

Aspectos que pueden aumentar la calidad en los estudios observacionales:

- Efecto importante: cuando el efecto observado muestra una asociación fuerte $(\mathrm{RR}>2 \mathrm{o}<0,5)$ o muy fuerte $(\mathrm{RR}>5 \mathrm{o}<0,2)$ y consistente, basada en estudios sin factores de confusión. En estas ocasiones se puede considerar la calidad como moderada o incluso alta.

- La presencia de un gradiente dosis-respuesta.

Situaciones en las cuales todos los posibles factores de confusión podrían haber reducido la asociación observada. En los casos en que el grupo de pacientes que reciben la intervención de interés presentan un peor pronóstico y, aún así, presentan mejores resultados que el grupo control, es probable que el efecto observado real sea mayor.

\section{C) Graduación de la fuerza de las recomendaciones}

La fuerza de las recomendaciones nos informa de en qué medida el llevar a cabo una recomendación conlleva más beneficios que riesgos. Existen una serie de factores que hay que tener en cuenta en la graduación de las recomendaciones:

- Balance entre beneficios y riesgos: Para realizar una adecuada valoración del balance entre los beneficios y los riesgos es necesario tener en cuenta el riesgo basal de la población a la que va dirigida la recomendación, y el efecto tanto en términos relativos como absolutos.

- Calidad de la evidencia científica: antes de llevar a cabo una recomendación es necesario conocer la certidumbre observada sobre la estimación del efecto. Si la calidad de la evidencia científica no es alta, a pesar de que la magnitud sea importante, debería disminuir la confianza y por tanto la fuerza con la que se lleve a cabo una recomendación.

- Valores y preferencias: la incertidumbre sobre los valores y las preferencias de la población diana a la cual va dirigida la GPC, será otro de los factores que hay que tener en cuenta. El personal sanitario, el colectivo de pacientes o la sociedad en general deben ver reflejados sus valores y sus preferencias que deberían influir en la graduación de las recomendaciones. 
- Costes: a diferencia de otras variables de resultado, los costes son mucho más variables en el tiempo, en distintas áreas geográficas y en función de diversas implicaciones. Así, aunque un coste elevado disminuye la probabilidad de graduar como fuerte una recomendación, el contexto será crítico en la valoración final.

La graduación de la fuerza de las recomendaciones considera dos categorías: recomendaciones fuertes y recomendaciones débiles.

En las recomendaciones fuertes el grupo elaborador confía en que los efectos beneficiosos superan a los perjudiciales o viceversa, que los daños superan a los beneficios. En el primer caso la recomendación es fuerte a favor. En el segundo es fuerte en contra. La recomendación se redacta con la expresión 'Se recomienda'.

Las recomendaciones débiles también pueden ser a favor o en contra. Una recomendación es débil a favor cuando el grupo elaborador concluye que los efectos beneficiosos de llevar a cabo la recomendación probablemente superan los perjudiciales, aunque no está completamente seguro. En cambio la recomendación es débil en contra, cuando los efectos adversos probablemente superan a los beneficiosos. La recomendación se redacta con la expresión 'Se puede valorar' o 'Se sugiere'.

Para las intervenciones de las que no se dispone de evidencia y el grupo elaborador quiere resaltar un determinado aspecto, se dispone de las recomendaciones de buena práctica clínica (BPC), basadas en la experiencia clínica y el consenso del grupo elaborador. 


\section{Anexo 2. Tratamientos farmacológicos}

En este anexo se presentan los tratamientos disponibles para la menopausia y postmenopausia, con el nombre del principio activo, el formato o presentación en la que está autorizada su comercialización en España y la dosis recomendada. Esta información de acceso gratuito puede consultarse en la base de datos del Consejo Oficial del Colegio de Farmacéuticos (http://www.portalfarma.com/home.nsf) y las fichas técnicas pueden consultarse en la web de la Agencia Española de Medicamentos y Productos Sanitarios (AEMPS. Ministerio de Sanidad, Servicios Sociales e Igualdad) (https://sinaem4.agemed.es/consaem/pestanias.do?metodo=presentacionInicial).

\section{Tratamientos farmacológicos abordados en esta guía}

En las tablas siguientes se relacionan los tratamientos farmacológicos que se abordan en esta guía. Se estructuran por subgrupos terapéuticos, según la clasificación ATC (Anatomical, Therapeutic, Chemical classification system) y vía de administración, todos los principios activos y sus presentaciones disponibles a fecha de abril de 2015. La información presentada es la necesaria para efectuar una prescripción por principio activo en receta. Adicionalmente, se recoge la dosis recomendada para cada principio activo, según forma farmacéutica.

\begin{tabular}{|c|c|}
\hline \multicolumn{2}{|c|}{ Grupo G03C: ESTROGENOS } \\
\hline \multicolumn{2}{|c|}{ Oral } \\
\hline $\begin{array}{l}\text { Estradiol } 1 \mathrm{mg}, 20 \text { comprimidos } \\
\text { Estradiol } 1 \mathrm{mg}, 28 \text { comprimidos } \\
\text { Estradiol } 2 \mathrm{mg}, 28 \text { comprimidos } \\
\text { Estradiol } 500 \text { mcg, } 100 \text { comprimidos }\end{array}$ & 1-2 mg / día \\
\hline Estrógenos conjugados 625 mcg, 28 comprimidos & $\begin{array}{l}\text { 0,625-1,25 mg / día en administración cíclica o } \\
\text { continua }\end{array}$ \\
\hline Tibolona 2,5 mg, 28 comprimidos & 2,5 mg / día \\
\hline \multicolumn{2}{|c|}{ Transdérmico y percutáneo } \\
\hline $\begin{array}{l}\text { Estradiol } 25 \mathrm{mcg} 8 \text { parches } \\
\text { Estradiol } 50 \mathrm{mcg} 8 \text { parches } \\
\text { Estradiol } 75 \mathrm{mcg} 8 \text { parches } \\
\text { Estradiol } 100 \mathrm{mcg} 8 \text { parches }\end{array}$ & $\begin{array}{l}1 \text { parche de } 50 \text { mcg / 3-4 días; modificar según } \\
\text { respuesta; dosis máxima de mantenimiento: } 1 \\
\text { parche de } 100 \text { mcg / día }\end{array}$ \\
\hline Estradiol 600 mcg / g 80 g gel & $\begin{array}{l}150 \text { mg / día en ciclos de } 3 \text { semanas y } 1 \text { de } \\
\text { descanso }\end{array}$ \\
\hline \multicolumn{2}{|c|}{ Vaginal } \\
\hline Estradiol 10 mcg, 18 comprimidos vaginales & $\begin{array}{l}\text { Inicialmente, } 10 \text { mcg / día durante } 2 \text { semanas; dosis } \\
\text { de mantenimiento: } 10 \text { mcg dos veces por semana. }\end{array}$ \\
\hline $\begin{array}{l}\text { Estriol } 500 \text { mcg, } 10 \text { óvulos } \\
\text { Estriol } 500 \mathrm{mcg}, 30 \text { óvulos }\end{array}$ & $\begin{array}{l}500 \text { mcg / día durante 2-3 semanas, } \\
\text { posteriormente } 500 \text { mcg / 2-3 días al acostarse. }\end{array}$ \\
\hline Estriol $1 \mathrm{mg} / \mathrm{g} 15 \mathrm{~g}$ crema vaginal & $\begin{array}{l}1 \text { aplicación / día durante 2-3 semanas, } \\
\text { posteriormente } 1 \text { aplicación / 2-3 días al acostarse. }\end{array}$ \\
\hline Promestrieno 10mg / g 30g crema vaginal & 1-2 aplicaciones / día durante 20 días. \\
\hline
\end{tabular}




\begin{tabular}{|c|c|}
\hline \multicolumn{2}{|c|}{ Grupo G03F: ESTRÓGENOS Y PROGESTÁGENOS } \\
\hline \multicolumn{2}{|c|}{ Oral } \\
\hline $\begin{array}{l}\text { Drospirenona } 2 \mathrm{mg} \text { / Estradiol } 1 \mathrm{mg}, 28 \\
\text { comprimidos } \\
\text { Drospirenona } 2 \mathrm{mg} \text { / Estradiol } 1 \mathrm{mg}, 84 \\
\text { comprimidos }\end{array}$ & 1 comprimido / día \\
\hline $\begin{array}{l}\text { Estradiol } 1 \mathrm{mg} \text { / Noretisterona } 500 \text { mcg, } 28 \\
\text { comprimidos }\end{array}$ & 1 comprimido / día \\
\hline $\begin{array}{l}\text { Estradiol } 500 \mathrm{mcg} / \text { Noretisterona } 100 \mathrm{mcg}, 28 \\
\text { comprimidos }\end{array}$ & 1 comprimido / día \\
\hline $\begin{array}{l}\text { Estradiol } 1 \mathrm{mg} / \text { Noretisterona } 1 \mathrm{mg}, 28 \\
\text { comprimidos }\end{array}$ & $\begin{array}{l}\text { Estradiol } 1 \mathrm{mg} \text { / día durante } 16 \text { días; Estradiol } \\
1 \mathrm{mg} \text { - Noretisterona } 1 \mathrm{mg} \text { / día durante } 12 \text { días }\end{array}$ \\
\hline $\begin{array}{l}\text { Estradiol } 1 \mathrm{mg} \text { y } 2 \mathrm{mg} \text { / Noretisterona } 1 \mathrm{mg}, 28 \\
\text { comprimidos }\end{array}$ & $\begin{array}{l}\text { Estradiol } 2 \text { mg / día durante } 12 \text { días; Estradiol } \\
2 \text { mg - Noretisterona } 1 \mathrm{mg} \text { / día durante } 10 \text { días; } \\
\text { Estradiol } 1 \mathrm{mg} \text { / día durante } 6 \text { días }\end{array}$ \\
\hline $\begin{array}{l}\text { Estradiol } 2 \text { mg - Norgestrel } 500 \text { mcg, } 21 \\
\text { comprimidos }\end{array}$ & $\begin{array}{l}\text { Estradiol } 2 \text { mg / día durante } 11 \text { días; Estradiol } \\
2 \mathrm{mg} \text { - Norgestrel } 500 \mathrm{mcg} \text { / día durante } 10 \text { días }\end{array}$ \\
\hline Estradiol 2 mg / Dienogest 2 mg, 28 comprimidos & 1 comprimido / día \\
\hline $\begin{array}{l}\text { Estradiol } 2 \mathrm{mg} / \text { Medroxiprogesterona 10mg, } 21 \\
\text { comprimidos }\end{array}$ & $\begin{array}{l}\text { Estradiol } 2 \text { mg / día durante } 11 \text { días; Estradiol } \\
2 \text { mg - Medroxiprogesterona } 10 \text { mg / día durante } \\
10 \text { días }\end{array}$ \\
\hline \multicolumn{2}{|c|}{ Transdérmico } \\
\hline $\begin{array}{l}\text { Estradiol } 50 \text { mcg / Noretisterona } 250 \text { mcg, } 8 \\
\text { parches }\end{array}$ & 1 parche / 3-4 días \\
\hline $\begin{array}{l}\text { Estradiol } 50 \mathrm{mcg} / \text { Estradiol } 50 \mathrm{mcg} \text { - } \\
\text { Noretisterona } 250 \mathrm{mcg} 8 \text { parches (fase I + fase II) }\end{array}$ & $\begin{array}{l}1 \text { parche estradiol } 50 \mathrm{mcg} \text { / 3-4 días durante } 14 ; 1 \\
\text { parche estradiol } 50 \mathrm{mcg} \text { / 3-4 días durante } 14 \\
\text { días }\end{array}$ \\
\hline
\end{tabular}




\section{Anexo 3. Principales guías de práctica clínica y otros recursos de utilidad}

Para la elaboración de la guía de práctica clínica sobre menopausia y postmenopausia se han tenido en cuenta algunas guías y consensos publicados sobre este tema como fuentes secundarias de bibliografía. A continuación se proporciona un listado y el enlace al texto completo de los principales documentos que pueden constituir una importante fuente de consulta para las personas usuarias de la presente guía.

\section{Guías de práctica clínica sobre menopausia}

Título: Menoguía. Asociación Española para el estúdio de la menopausia. 2012

\section{Enlace web:}

http://www.aeem.es/documentos/menoguias/MENOGUIAPERIMENOPAUSIA.pdf

Título: Management of Menopausal Symptoms Obstetrics \& gynecology. vol. 123, no. 1, january 2014.

\section{Enlace web:}

http://journals.lww.com/greenjournal/Abstract/2014/01000/Practice_Bulletin_No_141_ _Management_of.37.aspx

Título: The 2013 British Menopause Society \& Women's Health Concern recommendations on hormone replacement therapy

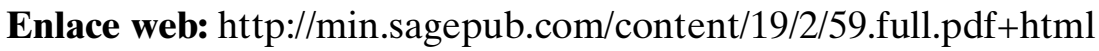

Título: Menopause and Osteoporosis Update 2009. SOGC Clinical Practice Guideline. JOGC. 2009:31(1):S1-46.

Enlace web: http://www.sogc.org/guidelines/

Título: Colegio Mexicano de Especialistas en Ginecología y Obstetricia. Estudio del climaterio y la menopausia. Ginecol Obstet Mex. 2009;77(9):S253-76.

Enlace web: http://www.comego.org.mx/

Título: Menopause and Hormone Therapy (HT): Collaborative Decision-Making and Management. Institute for Clinical Systems Improvement, 2008.

Enlace web: http://www.icsi.org/

Título: Nelson HD, Haney E, Humphrey L, Miller J, Nedrow A, Nicolaidis C, Vesco K, Walker M, Bougatsos C, Nygren P. Management of Menopause-Related Symptoms. Evidence Report/Technology Assessment No. 120. (Prepared by the Oregon Evidencebased Practice Center, under Contract No. 290-02-0024.) AHRQ Publication No. 05E016-2. Rockville, MD: Agency for Healthcare Research and Quality. March 2005.

Enlace web: http://www.ahrq.gov/clinic/tp/menopstp.htm 
Título: Grupo de trabajo de menopausia y postmenopausia. Guía de práctica clínica sobre la menopausia y postmenopausia. Barcelona: Sociedad Española de Ginecología y Obstetricia, Asociación Española para el Estudio de la Menopausia, Sociedad Española de Medicina de Familia y Comunitaria y Centro Cochrane Iberoamericano; 2004.

Enlace web: http://www.guiasalud.es/

\section{Consensos}

Título: Global Consensus Statement on menopausal hormone therapy. Maturitas 2013 Apr; 74(4):391-392.

\section{Enlace web:}

http://www.maturitas.org/article/S0378-5122 \%2813 \%2900046-7/pdf

Título: The North American Menopause Society. Estrogen and progestogen use in postmenopausal women: 2010 position statement of The North American Menopause Society. Menopause. 2010;17(2):242-55.

Enlace web: http://www.menopause.org/

Título: Gompel A, Rozenberg S, Barlow DH, the EMAS board members. The EMAS 2008 update on clinical recommendations on postmenopausal hormone replacement therapy. Maturitas. 2008;61:227-32.

Enlace web: http://www.emas-online.org/

Título: Board of the International Menopause Society. IMS Updated Recommendations on postmenopausal hormone therapy. Climacteric. 2007;10:181-94.

Enlace web: http://www.imsociety.org/

Título: American Association of Clinical Endocrinologists. Medical guidelines for clinical practice for the diagnosis and treatment of menopause. Endocr Pract. 2006;12(3):315-37.

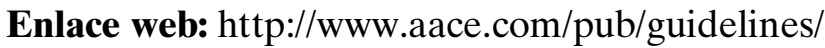

Título: National Institutes of Health State-of-the-Science Conference Statement on Management of Menopause-Related Symptoms. NIH Consensus and State-of-theScience Statements. 2005;22(1):1-44.

Enlace web: http://consensus.nih.gov/ 


\section{Anexo 4. Acrónimos y abreviaturas}

BPC: Buena práctica clínica.

DME: Diferencia de medias estandarizada.

DMP: Diferencias de medias ponderada.

ECA: $\quad$ Ensayo clínico aleatorizado.

GPC: Guía de Práctica Clínica.

GRADE: Grading of Recommendations of Assessment Development and Evaluations.

IC: $\quad$ Intervalo de confianza.

IK: Índice de Kupperman.

ITU: Infección del tracto urinario.

IMC: Índice de masa corporal.

MRS: $\quad$ Menopause Rating Scale.

NICE: $\quad$ National Institute for Clinical Excellence.

OR: $\quad$ Odds ratio.

PICO: Paciente, Intervención, Comparación y resultado (Outcome).

RR: $\quad$ Riesgo relativo.

RS: $\quad$ Revisión sistemática.

SERM: Moduladores selectivos de los receptores estrogénicos.

SIGN: $\quad$ Scottish Intercollegiate Guidelines Network.

SNS: $\quad$ Sistema Nacional de Salud. 



\section{Anexo 5. Glosario}

Climaterio: Periodo de la vida de la mujer que se extiende desde 2-8 años antes de la fecha de la menopausia hasta 2-6 años después de la última menstruación.

Menopausia: Cese permanente de la menstruación que resulta de la pérdida de la actividad folicular ovárica. Es la fecha de la última regla y suele ocurrir aproximadamente a los 50 años. Se reconoce tras 12 meses consecutivos de amenorrea, sin que exista ninguna otra causa patológica o fisiológica.

Menopausia artificial: Menopausia que ocurre como consecuencia de la extirpación de los ovarios, con o sin histerectomía, o por las radiaciones y la quimioterapia que causan destrucción de las células germinales.

Menopausia espontánea o natural: Menopausia que ocurre de forma gradual y progresiva por el normal envejecimiento ovárico.

Menopausia precoz (también llamada 'fallo ovárico prematuro'): Menopausia que ocurre antes de los 40 años.

Menopausia tardía: Menopausia que ocurre después de los 55 años.

Perimenopausia: Incluye el periodo anterior a la menopausia (cuando comienzan las manifestaciones endocrinológicas, biológicas y clínicas indicativas de que se aproxima la menopausia) y el primer año tras la menopausia.

Premenopausia: Incluye el periodo de 2-6 años previo a la menopausia, que puede cursar con sintomatología.

Postmenopausia establecida: Incluye el periodo a partir de los 12 meses de la fecha de última regla.

Postmenopausia inmediata: Incluye el periodo desde la última menstruación hasta un año después. 



\section{Anexo 6. Declaración de conflictos de interés}

La declaración de los intereses de los autores, revisores y colaboradores expertos ha sido llevada a cabo mediante un formulario predefinido incluido en el Manual Metodológico para la elaboración de Guías de Práctica Clínica del Sistema Nacional de Salud.

\section{Autores:}

Jacobo Cambil Martín, M. ${ }^{a}$ José Iglesias Piñeiro, Alberto López García-Franco, Laia Llort i Samsó, Emilia Bailón Muñoz, Victoria Jiménez Espínola, Juliana Ester Martín López, Teresa Molina López, Núria Nadal Braqué, Maria Teresa Nieto Rodríguez, Sonia Peláez Moya, Esther Redondo Margüello, Maria de las Nieves Respaldiza Salas, Mercedes Ricote Belinchon, Maria Ángeles Ruiz Alora, Dolores Ruiz Berdún, Juan José Sagarzazu Goenaga y Carmen Suárez Alemán han declarado ausencia de conflictos de interés.

Francesc Baró Mariné ha participado como ponente en conferencias organizadas por MSD (2009) y en cursos de formación continuada de Roche (2010). También ha recibido financiación para participar en estudios de MSD y Amgen (2009-10). Joaquín Calaf Alsina ha participado en actividades educacionales o de investigación clinica y formado parte de comités asesores de forma remunerada para las compañias: MSDOrganon, Bayer- Schering, Isdin, Lilly, Casen Fleet y Amgen. M. Jesús Cancelo Hidalgo ha participado como ponente en congresos nacionales y regionales por invitación de la organización de los mismos y en cursos de formación continuada en fitoterapia (2010). También ha recibido financiación para la elaboración de material docente para curso de formación continuada en fitoterapia por parte de la industria farmacéutica (2009). M. ${ }^{a}$ Jesús Cornellana Puigarnau ha recibido honorarios como ponente en varios cursos y simposiums. M. ${ }^{a}$ Dolores Juliá Mollá ha participado como ponente en congresos nacionales y locales (2009-10) y en un curso de formación continuada en fitoterapia (2010). También ha recibido financiación para la elaboración de material docente para curso de formación continuada en fitoterapia por parte de la industria farmacéutica (2009). M. ${ }^{a}$ Dolores Saavedra Llobregat ha recibido financiación para la asistencia a varios congresos por parte de los Laboratorios ARBORA/AUSONIA (2008-10) y honorarios como ponente en unas Jornadas de Enfermería (2008). Rafael Sánchez Borrego ha recibido financiación para realizar Cursos Diatros en Salud de la Mujer, por participar como ponente en varios cursos a nivel nacional e internacional y en varios ensayos clínicos. También ha sido miembro del Advisory Board de Amgen y Pfizer. 



\section{Bibliografía}

1. Nelson HD. Menopause. Lancet. 2008;371:760-70.

2. Jacobsen BK, Heuch I, Kvale G. Age at natural menopause and all-cause mortality: a 37-year follow-up of 19,731 Norwegian women. Am J Epidemiol. 2003;157:923-9.

3. Gold, EB, Colvin, A, Avis, N, et al. Longitudinal analysis of the association between vasomotor symptoms and race/ethnicity across the menopausal transition: study of women's health across the nation. Am J Public Health. 2006;96:1226-35.

4. De la Gándara J, Sánchez J, Díez MA, Monje E. Influencia de las actitudes, expectativas y creencias en la adaptación a la menopausia. Anales de Psiquiatría. 2003;8:329-336.

5. Melby MK, Lock M, Kaufert P. Culture and symptom reporting at menopause. Hum Reprod Update. 2005;11:495-512.

6. Sommer B, Avis N, Meyer P, Ory M, Madden T, Kagawa-Singer M, et al. Attitudes toward menopause and aging across ethnic/racial groups. Psychosom Med. 1999; 61:868-875.

7. Dennerstein L, Dudley EC, Hopper JL, Guthrie JR, Burger HG. A prospective population-based study of menopausal symptoms. Obstet Gynecol. 2000;96:351-358.

8. Robinson D, Cardozo L. The menopause and HRT. Urogenital effects of hormone therapy. Best Pract Res Clin Endocrinol Metab. 2003;17:91-104.

9. Group for the Women's Health Initiative Investigators. Risk and Benefits of Estrogen Plus Progestin in Healthy Postmenopausal Women. Principal Results from the women's Health Inititive Randomized Controlled Trial. JAMA. 2002;288:321-333.

10. Grupo de trabajo sobre GPC. Elaboración de Guías de Práctica Clínica en el Sistema Nacional de Salud. Manual Metodológico. Madrid: Plan Nacional para el SNS del MSC. Instituto Aragonés de Ciencias de la SaludI+CS; 2007. Guías de Práctica Clínica en el SN.

11. Grupo de trabajo de menopausia y postmenopausia. Guía de práctica clínica sobre la menopausia y postmenopausia. Barcelona: Sociedad Española de Ginecología y Obstetricia, Asociación Española para el Estudio de la Menopausia, Sociedad Española de Medicina Familiar y Comunitaria. 
12. Schünemann HJ, Jaeschke R, Cook DJ, Bria WF, El-Solh AA, Ernst A, et al. ATS Documents Development and Implementation Committee. An official ATS statement: grading the quality of evidence and strength of recommendations in ATS guidelines and recommendations. Am J Respir Crit Care Med. 2006 Sep 1;174:605-14.

13. World Health Organization. Research on the menopause in the 1990s. WHO Tech Rep Ser No. 866. Geneva: WHO, 1996.

14. Cuadros J, Llaneza P, Mateu S. Demografía y epidemiología del climaterio en España. En Comino R. Ed. Libro Blanco de la Menoapusia en España. EMISA Ed. Madrid 2000;13-33.

15. Gold EB, Bromberger J, Crawford S, Samuels S, Greendale GA, Harlow $\mathrm{SD}$ et al. Factors associated with age at natural menopause in a multiethnic sample of midlife women. Am J Epidemiol. 2001;153:865-74.

16. Greene JG. A factor analytic study of climacteric symptoms. J Psychosom Res. 1976;20:425-30.

17. Kupperman HS, Blatt MHG, Wiesbader H, Filler W: Comparative clinical evaluation of estrogen preparations by the menopausal and amenorrhoea indices. J Clin Endocrinol. 1953,13:688-703.

18. Kupperman HS, Wetchler BB, Blatt MHG: Contemporary therapy of the menopausal syndrome. JAMA. 1959,171:1627-37.

19. Hilditch JR, Lewis J, Peter A, van Maris B, Ross A, Franssen E et al. A menopause-specific quality of life questionnaire: development and psychometric properties. Maturitas. 1996;24:161-75.

20. Palacios S, Ferrer-Barriendos J, Parrilla JJ, Castelo-Branco C, Manubens $\mathrm{M}$, Alberich $\mathrm{X}$ et al. [Health-related quality of life in the Spanish women through and beyond menopause. Development and validation of the Cercantes Scale]. Med Clin (Barc). 2004;122:205-11.

21. Stearns V, Ullmer L, Lopez JF, Smith Y, Isaacs C, Hayes DF. Hot flushes. Lancet. 2002;360:1851-1861.

22. Erlik Y, Tataryn IV, Meldrum DR, Lomax P, Bajorek JG, Judd HL. Association of waking episodes with menopausal hot flushes. JAMA. 1981;245:1741-4.

23. Huang AJ, Grady D, Jacoby VL, Blackwell TL, Bauer DC, Sawaya GF. Persistent hot flushes in older postmenopausal women. Arch Intern Med. 2008;168:840-6.

24. Politi MC, Schleinitz MdD, Col NF. Revisiting the duration of vasomotor symptoms of menopause: a meta-analysis. J Gen Intern Med. 2008;23:1507-13.

25. Freeman EW, Sammel MD, Lin H, Gracia CR, Pien GW, Nelson DB et al. Symptoms associated with menopausal transition and reproductive hormones in midlife women. Obstet Gynecol. 2007;110(2 Pt 1):230-40. 
26. Brown WJ, Mishra GD, Dobson A. Changes in physical symptoms during the menopause transition. Int J Behav Med. 2002; 9:53-67.

27. Hardy R, Kuh D. Change in psychological and vasomotor symptom reporting during the menopause. Soc Sci Med. 2002;55:1975-88.

28. Haas S, Schiff I. Síntomas de déficit de estrógenos. En Studd JWW, Whitehead M, editors. Menopausia. Barcelona: Carlos Alejandra editor, 1990; 17-26.

29. Dennerstein L, Guthrie J, Birkhäuser M, Sherman S. Symptoms and the menopause. International Position Paper on Women's Health and Menopause: A comprehensive Approach. National Institutes of Health 2002; 43-63.

30. Mohyi D, Tabassi K, Simon J. Differential diagnosis of hot flashes. Maturitas. 1997;27:203-14.

31. Robinson D, Cardozo L. The menopause and HRT. Urogenital effects of hormone therapy. Best Pract Res Clin Endocrinol Metab. 2003;17:91-104.

32. Grady D. Management of menopausal symptoms. New England Journal of Medicine. 2006;355:2338-47.

33. Foxman B, Barlow R, D'Arcy H, Guillespie B, Sobel JD. Urinary tract infection: self reported incidence and associated costs. Annals of Epidemiology. 2000;10:509-15.

34. Raz R, Gennesin Y, Wasser J, Stoler Z, Rosenfeld S, Rottensterich E, et al.Recurrent urinary tract infections in postmenopausal women. Clinical Infectious Diseases. 2000;30:152-6.

35. Sherburn M, Guthrie JR, Dudley EC, O'Connell HE, Dennerstein L. Is incontinence associated with menopause? Obstetrics \& Gynecology. 2001:98:628-33.

36. Cody JD, Richardson K, Moehrer B, Hextall A, Glazener CMA. Oestrogen therapy for urinary incontinence in post-menopausal women. Cochrane Database of Systematic Reviews 2009, Issue 4. Art. No.: CD001405. DOI:10.1002/14651858.CD001405.pub2.

37. Hickey M, Davis SR, Sturdee DW. Treatment of menopausal symptoms: what shall we do now? Lancet. 2005;366:409-21.

38. Nelson H, Haney E, Humphrey L, et al. Management of MenopauseRelated Symptoms: Evidence Report/Technology Assessment No. 120. Rockville, Md: Agency for Healthcare Research and Quality; 2005.

39. Rees M, Purdie DW (Eds.). Management of the menopause: the handbook. 4th edn. London/Marlow, 2006: Royal Society of Medicine Press Ltd \& British Menopause Society Publications Ltd. 
40. Roberts H. Managing the menopause. BMJ. 2007:334:736-41.

41. Avis NE, Bramblia D, MacKinlay SM, Vass K. A longitudinal analysis of the association between menopause and depression: the results from the Massachusetts Women's Health Study. Ann Epidemiol 1994;4:214-20.

42. Dennerstein L, Guthrie J, Clark M, Lehert P, Henderson V. A populationbased study of depressed mood in middle-aged Australian-born women. Menopause. 2004;11:563-8.

43. Vesco KK, Haney EM, Humphrey L, Fu R, Nelson HD. Influence of menopause on mood: a systematic review of cohort studies. Climacteric. 2007;10:448-65.

44. Dennerstein L, Lehert P, Dudley E, Guthrie J. Factors contributing to positive mood during the menopausal transition. J Nerv Ment Dis. 2001;189:84-89.

45. Freeman E, Samuel M, Lin H, Nelson D. Associations of hormones and menopausal status with depressed mood in women with no history of depression. Arch Gen Psychiatry. 2006;63:375-82.

46. Cohen L, Soares C, Vitonis A, Otto M, Harlow B. Risk of new onset of depression during the menopausal transition: the Harvard Study of Moods and Cycles. Arch Gen Psychiatry. 2006;63:385-90.

47. Bromberger J, Matthews K, Schott LL, Brockwell S, Avis NE, Kravitz HM, et al. Depressive symptoms during the menopausal transition: the Study of Women's Health Across the Nation (SWAN). J Affect Disord. 2007;103:267-72.

48. Woods NF, Smith-DiJulio K, Percival DB, Tao EY, Mariella A, Mitchell ES. Depressed mood during the menopausal transition and early postmenopause: observations from the Seattle Midlife Women's Health study. Menopause. 2008;15:223-32.

49. Maartens LW, Knottnerus JA, Pop VJ. Menopausal transition and increased depressive symptomatology: a community-based prospective study. Maturitas. 2002;42:195-200.

50. Yaffe K, Sawaya G, Lieberburg I, Grady D. Estrogen therapy in postmenopausal women. Journal of the American Medical Association. 1998;279:688-695.

51. Hogervorst E, Williams J, Budge M, Riedel W, Jolles J. The nature of the effect of female gonadal hormone replacement therapy on cognitive function in post-menopausal women: a meta-analysis. Neuro-science. 2000;101:485-512.

52. Lethaby A, Hogervorst E, Richards M, Yesufu A, Yaffe K. Hormone replacement therapy for cognitive function in postmenopausal women. Cochrane Database of Systematic Reviews 2008, Issue 1. Art. No.: CD003122. DOI:10.1002/14651858.CD003122.pub2. 
53. Hogervorst E, Yaffe K, Richards M, Huppert FAH. Hormone replacement therapy to maintain cognitive function in women with dementia. Cochrane Database of Systematic Reviews 2009, Issue 1. Art. No.: CD003799. DOI:10.1002/14651858.CD003799.pub2.

54. Maki PM, Sundermann E. Hormone therapy and cognitive function. Human Reproduction Update. 2009;15:667-81.

55. Dennerstein L, Dudley E, Burger H. Are changes in sexual functioning during midlife due to aging or menopause? Fertil Steril. 2001;76:456-60.

56. Dennerstein L, Randolph J, Taffe J, Dudley E, Burger H. Hormones, mood, sexuality and the menopausal transition. Fertil Steril. 2002;77(suppl 4):S42-8.

57. Dennerstein L, Lehert P, Burger H, Guthrie J. Sexuality. Am J Med. 2005;118(suppl 2):59-63.

58. Panay N, Dutta R, Ryan A. Crash course: obstetrics and gynaecology. Edinburgh, 2004: Mosby.

59. Lauritzen C, Studd J. (Eds.) Current management of the menopause. Abingdon, Oxon, 2005: Taylor \& Francis.

60. The Society of Obstetricians and Gynaecologists of Canada. Menopause and osteoporosis update 2009. JOGC. 2009;31(suppl 1):1-46.

61. Ferrara CM, Lynch NA, Nicklas BJ, Ryan AS, Berman DM. Differences in adipose tissue metabolism between postmenopausal and perimenopausal women. J Clin Endocrinol Metab 2002;87:4166-70.

62. Toth MJ, Tchernof A, Sites CK, Poehlman ET. Effect of menopausal status on body composition and abdominal fat distribution. Int $\mathrm{J}$ Obes Relat Metab Disord. 2000;24:226-31.

63. Poehlman ET, Toth MJ, Gardner AW. Changes in energy balance and body composition at menopause: a controlled longitudinal study. Ann Intern Med. 1995;123:673-75.

64. Crawford SL, Casey VA, Avis NE, McKinlay SM. A longitudinal study of weight and the menopause transition: results from the Massachusetts Women's Health Study. Menopause. 2000;7:96-104.

65. Gold EB, Sternfeld B, Kelsey JL, Brown C, Mouton C, Reame N, et al. Relation of demographic and lifestyle factors to symptoms in a multiracial/ethnic population of women 40-55 years of age. Am J Epidemiol [Internet]. 2000 Sep 1 [cited 2014 Sep 29];152:463-73. Available from: http://www.ncbi.nlm.nih.gov/pubmed/10981461

66. Gold EB, Block G, Crawford S, Lachance L, FitzGerald G, Miracle H, et al. Lifestyle and demographic factors in relation to vasomotor symptoms: baseline results from the Study of Women's Health Across the Nation. Am J Epidemiol [Internet]. 2004 Jun 15 [cited 2014 Sep 29];159(12):1189-99. Available from: http://www.ncbi.nlm.nih.gov/pubmed/15191936 
67. Greendale GA, Gold EB. Lifestyle factors: are they related to vasomotor symptoms and do they modify the effectiveness or side effects of hormone therapy? Am J Med [Internet]. 2005 Dec 19 [cited 2014 Sep 22];118 Suppl 148-54. Available from: http://www.ncbi.nlm.nih.gov/pubmed/16414341

68. Thurston RC, Sowers MR, Chang Y, Sternfeld B, Gold EB, Johnston JM, et al. Adiposity and reporting of vasomotor symptoms among midlife women: the study of women's health across the nation. Am J Epidemiol [Internet]. 2008 Jan 1 [cited 2014 Sep 29];167(1):78-85. Available from: http://www.ncbi.nlm.nih.gov/ pubmed/17881385

69. Thurston RC, Sowers MR, Sternfeld B, Gold EB, Bromberger J, Chang Y, et al. Gains in body fat and vasomotor symptom reporting over the menopausal transition: the study of women's health across the nation. Am J Epidemiol [Internet]. 2009 Sep 15 [cited 2014 Sep 21];170(6):766-74. Available from: http://www.pubmedcentral.nih.gov/articlerender.fcgi? $\operatorname{artid}=2768523 \&$ tool $=p$ mcentrez\&rendertype $=$ abstract

70. Thurston RC, Sowers MR, Sutton-Tyrrell K, Everson-Rose SA, Lewis TT, Edmundowicz D, et al. Abdominal adiposity and hot flashes among midlife women. Menopause [Internet]. [cited 2014 Sep 21];15(3):429-34. Available from: http://www.pubmedcentral.nih.gov/articlerender.fcgi? $\operatorname{artid}=2875947 \&$ tool $=$ pm centrez\&rendertype $=$ abstract

71. Huang AJ, Subak LL, Wing R, West DS, Hernandez AL, Macer J, Grady D; Program to Reduce Incontinence by Diet and Exercise Investigators. An intensive behavioral weight loss intervention and hot flushes in women. Arch Intern Med. 2010;170:1161-7.

72. Daley A, Stokes-Lampard H, Mutrie N, MacArthur C. Exercise for vasomotor menopausal symptoms. Cochrane Database of Systematic Reviews 2007, Issue 4. Art. No.: CD006108. DOI: 10.1002/14651858.CD006108.pub2.

73. Lindh-Åstrand L, Nedstrand E, Wyon, et al.Vasomotor symptoms and quality of life in previously sedentary postmenopausal women randomised to activity or estrogen therapy. Maturitas. 2003;48:97-105.

74. Moriyama CK, Oneda B, Bernardo FR, et al. A randomized, placebocontrolled trial of the effects of physical exercises and estrogen therapy on health-related quality of life in postmenopausal women. Menopause. 2008;15:613-8.

75. Luoto R, Moilanen J, Heinonen R, Mikkola T, Raitanen J, Tomas E, Ojala K, Mansikkamäki K, Nygård $\mathrm{CH}$. Effect of aerobic training on hot flushes and quality of life--a randomized controlled trial. Ann Med. 2012;44:616-26.

76. Moilanen JM, Mikkola TS, Raitanen JA, Heinonen RH, Tomas EI, Nygård $\mathrm{CH}$, Luoto RM. Effect of aerobic training on menopausal symptoms--a randomized controlled trial. Menopause. 2012;19:691-6. 
77. Mansikkamäki K, Raitanen J, Nygård $\mathrm{CH}$, Heinonen R, Mikkola T, EijaTomás, Luoto R. Sleep quality and aerobic training among menopausal women--a randomized controlled trial. Maturitas. 2012;72:339-45.

78. Lee MS, Kim JI, Ha JY, Boddy K, Ernst E. Yoga for menopausal symptoms: a systematic review. Menopause. 2009; 16:602-8.

79. Stearns V, Ullmer L, Lopez JF, Smith Y, Isaacs C, Hayes DF. Hot flushes. Lancet. 2002;360:1851-1861.

80. MacLennan AH, Broadbent JL, Lester S, Moore V. Oral oestrogen and combined oestrogen/progestogen therapy versus placebo for hot flushes. Cochrane Database of Systematic Reviews 2004, Issue 4. Art. No.: CD002978. DOI: 10.1002/14651858.CD002978.pub2.

81. Nelson HD, Miranda W, Zakher B, Mitchell J. Menopausal hormone therapy for the primary prevention of chronic conditions: a systematic review to update the U.S. Preventive Services Task Force recommendations. Ann Intern Med. 2012; 157: 104-13.

82. Marjoribank J, Farquhar C, Roberts H, Lethaby A. Long term hormone therapy for perimenopausal and postmenopausal women. Cochrane Database of Systematic Reviews. 2012, issue 7. Art. No.: CD004143. DOI: 10.1002/ 14651858.CD004143.pub4.

83. Greiser CM, Greiser EM, Dören M. Menopausal hormone therapy and risk of lung cancer-Systematic review and meta-analysis. Maturitas. 2010; 65: 198-204.

84. Liu JH, Reape KZ, Hait HI. Synthetic conjugated estrogens-b and postmenopausal nocturnal vasomotor symptoms: A randomized controlled trial. Obstet Gynecol. 2012;119(1):78-84.

85. Brunner RL, Aragaki A, Barnabei V, Cochrane BB, Gass M, Hendrix S, et al. Menopausal symptom experience before and after stopping estrogen therapy in the Women's Health Initiative randomized, placebo-controlled trial. Menopause. 2010;17(5):946-54.

86. Speroff L, Haney AF, Gilbert RD, Ellman H; Estradiol Acetate Investigator Group. Efficacy of a new, oral estradiol acetate formulation for relief of menopause symptoms. Menopause. 2006;13:442-50.

87. Utian WH, Speroff L, Ellman H, Dart C. Comparative controlled trial of a novel oral estrogen therapy, estradiol acetate, for relief of menopause symptoms. Menopause. 2005;12:708-15.

88. Liu J, Allgood A, Derogatis LR, Swanson S, O'Mahony M, Nedoss B et al. Safety and efficacy of low-dose esterified estrogens and methyltestosterone, alone or combined, for the treatment of hot flashes in menopausal women: A randomized, double-blind, placebo-controlled study. Fertil Steril. 2011 Jan;95:366-8. Epub 2010 Sep 17. 
89. Cieraad D, Conradt C, Jesinger D, Bakowski M. Clinical study comparing the effects of sequential hormone replacement therapy with oestradiol/dydrogesterone and conjugated equine oestrogen/norgestrel on lipids and symptoms. Arch Gynecol Obstet. 2006;274:74-80.

90. Endrikat J, Graeser T, Mellinger U, Ertan K, Holz C. A multicenter, prospective, randomized, double-blind, placebo-controlled study to investigate the efficacy of a continuous-combined hormone therapy preparation containing $1 \mathrm{mg}$ estradiol valerate/ $2 \mathrm{mg}$ dienogest on hot flushes in postmenopausal women. Maturitas. 2007; 58:201-7.

91. Gambacciani M, Spielmann D, Genazzani AR. Efficacy on climacteric symptoms of a continuous combined regimen of $1 \mathrm{mg}$ 17beta-estradiol and trimegestone versus two regimens combining 1 or $2 \mathrm{mg}$ 17beta-estradiol and norethisterone acetate. Gynecol Endocrin. Gynecol Endocrinol. 2005;21:65-73.

92. Mattsson LA, Skouby SO, Heikkinen J, Vaheri R, Mäenpää J, Timonen U. A low-dose start in hormone replacement therapy provides a beneficial bleeding profile and few side-effects: randomized comparison with a conventional-dose regimen. Climacteric. 2004;7:59-69.

93. Schürmann R, Holler T, Benda N. Estradiol and drospirenone for climacteric symptoms in postmenopausal women: a double-blind, randomized, placebocontrolled study of the safety and efficacy of three dose regimens. Climacteric. 2004;7:189-96.

94. Yu H, Racketa J, Chines AA, Mirkin S. Hot flush symptom-free days with bazedoxifene/conjugated estrogens in postmenopausal women. Climacteric. 2013;16:252-7.

95. Archer DF, Pickar JH, MacAllister DC, Warren MP. Transdermal estradiol gel for the treatment of symptomatic postmenopausal women. Menopause. 2012;19:622-9.

96. Simon JA, Bouchard C, Waldbaum A, Utian W, Zborowski J, Snabes MC. Low dose of transdermal estradiol gel for treatment of symptomatic postmenopausal women: a randomized controlled trial. Obstet Gynecol. 2007;109:588-96.

97. Hedrick RE, Ackerman RT, Koltun WD, Halvorsen MB, Lambrecht LJ. Transdermal estradiol gel $0.1 \%$ for the treatment of vasomotor symptoms in postmenopausal women. Menopause. 2009;16:132-40.

98. Haines C, Yu SL, Hiemeyer F, Schaefers M. Micro-dose transdermal estradiol for relief of hot flushes in postmenopausal Asian women: a randomized controlled trial. Climacteric. 2009;12:419-26.

99. Bachmann GA, Schaefers M, Uddin A, Utian WH. Lowest effective transdermal 17beta-estradiol dose for relief of hot flushes in postmenopausal women: a randomized controlled trial. Obstet Gynecol. 2007;110:771-9. 
100. Group for the Women's Health Initiative Investigators. Risk and Benefits of Estrogen Plus Progestin in Healthy Postmenopausal Women. Principal Results from the women's Health Inititive Randomized Controlled Trial. JAMA. 2002;288:321-333.

101. Davis SR, Davison SL, Wilson S, Shepherd J, Lawton B. Intranasal versus transdermal matrix oestrogen replacement in Australasian women. Maturitas. 2005;51:163-71.

102. Odabasi AR, Yuksel H, Demircan SS, Kacar DF, Culhaci N, Ozkara EE. A prospective randomized comparative study of the effects of intranasal and transdermal $17 \beta$-estradiol on postmenopausal symptoms and vaginal cytology. J Postgrad Med. 2007;53:221-7.

103. Studd J, Pornel B, Marton I, Bringer J, Varin C, Tsouderos Y, et al. Efficacy and acceptability of intranasal 17 beta-oestradiol for menopausal symptoms: randomised dose-response study. Aerodiol Study Group. Lancet. 1999; 353: 1574-8.

104. Panay N, Toth K, Pelissier C, Studd J. Dose-ranging studies of a novel intranasal estrogen replacement therapy. Maturitas. 2001;38 Suppl 1:S15-22.

105. Mattsson LA, Christiansen C, Colau JC, Palacios S, Kenemans P, Bergeron $\mathrm{C}$, et al. Clinical equivalence of intranasal and oral 17beta-estradiol for postmenopausal symptoms. Am J Obstet Gynecol. 2000;182:545-552.

106. Doren M, Azzawi FA, Donnez J, Van der Mooren MJ, Villero J, Gompel A. Therapeutic value and long-term safety of pulsed estrogen therapy. Maturitas. 2001;38 Suppl 1: S23-30.

107. Ozsoy M, Oral B, Ozsoy D. Clinical equivalence of intranasal estradiol and oral estrogens for postmenopausal symptoms. Int J Gynaecol Obstet. 2002;79:143-6.

108. Gordon SF, Thompson KA, Ruoff GE, Imig JR, Lane PJ, Schwenker CE. Efficacy and safety of a seven-day, transdermal estradiol drug-delivery system: comparison with conjugated estrogens and placebo. The Transdermal Estradiol Patch Study Group. Int J Fertil Menopausal Stud. 1995;40:126-34.

109. Studd JW, McCarthy K, Zamblera D, Burger HG, Silberberg S, Wren B, et al. Efficacy and tolerance of Menorest compared to Premarin in the treatment of postmenopausal women. A randomised, multicentre, doubleblind, double-dummy study. Maturitas. 1995;22:105-14.

110. Pornel B. Efficacy and safety of Menorest in two positive-controlled studies. Eur J Obstet Gynecol Reprod Biol. 1996;64 Suppl:S35-7.

111. Good WR, John VA, Ramirez M, Higgins JE. Comparison of Alora estradiol matrix transdermal delivery system with oral conjugated equine estrogen therapy in relieving menopausal symptoms. Alora Study Group. Climacteric. 1999;2:29-36. 
112. De Franciscis P, Cobellis L, Fornaro F, Sepe E, Torella M, Colacurci N. Low-dose hormone therapy in the perimenopause. Int J Gynaecol Obstet. 2007;98:138-42.

113. Drapier-Faure E, Azoulay C, Abramovici Y. Acceptability of continuous combined versus cyclical HRT: a French multicentric randomized clinical study. Gynecol Obstet Fertil. 2005;33:498-504.

114. Cameron ST, Glasier AF, Gebbie A, Dewart H, Baird DT. Comparison of a transdermal continuous combined and an interrupted progestogen HRT. Maturitas. 2006;53:19-26.

115. Von Holst T, Salbach B. Efficacy of a new 7-day transdermal sequential estradiol/levonorgestrel patch in women. Maturitas. 2002;41:231-42.

116. Cunha EP, Azevedo LH, Pompei LM, Strufaldi R, Steiner ML, Ferreira JA, et al. Effect of abrupt discontinuation versus gradual dose reduction of postmenopausal hormone therapy on hot flushes. Climacteric. 2010;13:362-7.

117. Lindh-Astrand L, Bixo M, Hirschberg AL, Sundström-Poromaa I, Hammar M. A randomized controlled study of taper-down or abrupt discontinuation of hormone therapy in women treated for vasomotor symptoms. Menopause. 2010;17:72-9.

118. Aslan E, Bagis T, Kilicdag EB, Tarim E, Erkanli S, Kuscu E. How best is to discontinue postmenopausal hormone therapy: immediate or tapered? Maturitas. 2007;56:78-83.

119. Haimov-Kochman R, Barak-Glantz E, Arbel R, Leefsma M, Brzezinski A, Milwidsky A, et al. Gradual discontinuation of hormone therapy does not prevent the reappearance of climacteric symptoms: a randomized prospective study. Menopause. 2006;13:370-6.

120. Lethaby A, Marjoribanks J, Fronenberg F, Roberts H, Eden J, Brown J. Phytoestrogens formenopausal vasomotor symptoms. Cochrane Database of Systematic Reviews 2013, Issue 12. Art. No.: CD001395. DOI: 10.1002/14651858.CD001395.pub4.

121. Hogervorst E, Yaffe K, Richards M, Huppert FAH. Hormone replacement therapy to maintain cognitive function in women with dementia. Cochrane Database of Systematic Reviews 2009, Issue 1. Art. No.: CD003799. DOI:10.1002/14651858.CD003799.pub2.

122. Farquhar C, Marjoribanks J, Lethaby A, Suckling JA, Lamberts Q. Long term hormone therapy for perimenopausal and postmenopausal women. Cochrane Database of Systematic Reviews 2009, Issue 2. Art. No.: CD004143. DOI: 10.1002/14651858.CD004143.pub3.

123. Shah NR, Borenstein J, Dubois RW. Postmenopausal hormone therapy and breast cancer: a systematic review and meta-analysis. Menopause. 2005; 12:668-78. 
124. Greiser CM, Greiser EM, Doren M. Menopausal hormone therapy and risk of ovarian cancer: systematic review and meta-analysis. Hum Reprod Update. 2007; 13:453-63.

125. Furness S, Roberts H, Marjoribanks J, Lethaby A, Hickey M, Farquhar C. Hormone therapy in postmenopausal women and risk of endometrial hyperplasia. Cochrane Database of Systematic Reviews 2009, Issue 2. Art. No.:CD000402. DOI: 10.1002/14651858.CD0004.

126. Sare GM, Gray LJ, Bath PM. Association between hormone replacement therapy and subsequent arterial and venous vascular events: a metaanalysis. Eur Heart J. 2008;29:2031-41.

127. Canonico M, Plu-Bureau G, Lowe GD, Scarabin PY. Hormone replacement therapy and risk of venous thromboembolism in postmenopausal women: systematic review and meta-analysis. BMJ. 2008; 336:1227-31.

128. Kongnyuy EJ, Norman RJ, Flight IHK, ReesMC. Oestrogen and progestogen hormone replacement therapy for peri-menopausal and postmenopausal women: weight and body fat distribution. Cochrane Database of Systematic Reviews 1999, Issue 3. Art. No.: CD0010.

129. Greising SM, Baltgalvis KA, Lowe DA, Warren GL. Hormone therapy and skeletal muscle strength: a meta-analysis. J Gerontol A Biol Sci Med Sci. 2009; 64:1071-81.

130. Allen IE, Monroe M, Connelly J, Cintron R, Ross SD. Effect of postmenopausal hormone replacement therapy on dental outcomes: systematic review of the literature and pharmacoeconomic analysis. Managed Care Interface. 2000;13:93-9.

131. The North American Menopause Society. Estrogen and progestogen use in postmenopausal women: 2010 position statement of The North American Menopause Society. Menopause. 2010;17:242-55.

132. Bullock JL, Massey FM, Gambrell D Jr. Use of medroxyprogesterone acetate to prevent menopausal symptoms. Obstetrics and Gynecology. 1975;46:165-8.

133. Morrison JC, Martin DC, Blair RA, Anderson GD, Kincheloe BW, Bates $\mathrm{GW}$, et al. The use of medroxyprogesterone acetate for relief of climacteric symptoms. Am J Obstet Gynecol. 1980;138:99-104.

134. Schiff I, Tulchinsky D, Cramer D, Ryan KJ. Oral medroxyprogesterone in the treatment of postmenopausal symptoms. JAMA. 1980;244:1443-5.

135. Albrecht BH, Schiff I, Tuchinsky D, Ryan KJ. Objective evidence that placebo and oral medroxyprogesterone acetate therapy diminish menopausal vasomotor flushes. Am J Obstet Gynecol. 1981;139:631-5. 
136. Loprinzi CL, Michalak JC, Quella SK, O'Fallon JR, Hatfield Ak, Nelimark R A, et al. Megestrol acetate for the prevention of hot flashes. N Engl J Med. 1994:331:347-52.

137. Aslaksen K, Frankendal B. Effect of oral medroxyprogesterone acetate on menopausal symptoms in patients with endometrial carcinoma. Acta Obstet Gynecol Scand. 1982;61:423-8.

138. Spark MJ, Willis J. Systematic review of progesterone use by midlife and menopausal women. Maturitas. 2012. p. 192-202.

139. Prior JC, Hitchcock CL. Progesterone for vasomotor symptoms: a 12-week randomized, masked placebo-controlled trial in healthy, normal-weight women 1-20 years since final menstrual flow. Endocrine Rev. 2010;31(Suppl. 1):S51.

140. Hitchcock CL, Prior JC. Oral micronized progesterone for vasomotor symptoms-a placebo-controlled randomized trial in healthy postmenopausal women. Menopause. 2012; 19:886-93.

141. Prior JC, Hitchcock CL. Progesterone for hot flush and night sweat treatment effectiveness for severe vasomotor symptoms and lack of withdrawal rebound. Gynecol Endocrinol 2012;28(Suppl. 2):7-11.

142. Lobo RA, McCormick W, Singer F, Roy S. Depo-medroxyprogesterone acetate compared with conjugated estrogens for the treatment of postmenopausal women. Obstet Gynecol. 1984;63:1-5.

143. Prior JC, Nielsen JD, Hitchcock CL, Williams LA, Vigna YM, Dean CB. Medroxyprogesterone and conjugated oestrogen are equivalent for hot flushes: a 1-year randomized double-blind trial following premenopausal ovariectomy. Clin Sci (Lond). 2007;112.

144. Benster B, Carey A, Wadsworth F, Vashisht A, Domoney C, Studd J. A double-blind placebo-controlled study to evaluate the effect of progestelle progesterone cream on postmenopausal women. Menopause Int. 2009;15:63-9.

145. Somboonporn W, Bell RJ, Davis SR. Testosterone for peri and postmenopausal women. Cochrane Database of Systematic Reviews 2005, Issue 4. Art. No.: CD004509. DOI: 10.1002/14651858.CD004509.pub2.

146. Matthews KA, Owens JF, Salomon K, Harris KF, Berga SL. Influence of hormone therapy on the cardiovascular responses to stress of postmenopausal women. Biological Psychology. 2005;69:39-56.

147. Warnock JK, Swanson SG, Borel RW, Zipfel LM, Brennan JJ. Combined esterified estrogens andmethyltestosterone versus esterified estrogens alone in the treatment of loss of sexual interest in surgically menopausal women. Menopause. 2005;12:374-84.

148. Rymer J, Morris EP. Extracts from "Clinical evidence": Menopausal symptoms. BMJ. 2000;321:1516-9. 
149. Modelska K, Cummings S. Tibolone for postmenopausal women: systematic review of randomized trials. J Clin Endocrinol Metab. 2002;87:16-23.

150. Landgren MB, Bennink HJ, Helmond FA, Engelen S. Dose-response analysis of effects of tibolone on climacteric symptoms. BJOG. 2002;109:1109-14.

151. Hammar ML, van de Weijer P, Franke HR, Pornel B, von Mauw EM, Nijland EA. Tibolone and low-dose continuous combined hormone treatment: vaginal bleeding pattern, efficacy and tolerability. BJOG. 2007;114:1522-9.

152. Somunkiran A, Erel CT, Demirci F, Senturk ML. The effect of tibolone versus 17 beta-estradiol on climacteric symptoms in women with surgical menopause: a randomized, cross-over study. Maturitas. 2007;56:61-8.

153. Swanson SG, Drosman S, Helmond FA, Stathopoulos VM. Tibolone for the treatment of moderate to severe vasomotor symptoms and genital atrophy in postmenopausal women: a multicenter, randomized, double-blind, placebo-controlled study. Menopause. 2006;13:917-25.

154. Meeuwsen IB, Samson MM, Duursma SA, Verhaar HJ. The influence of tibolone on quality of life in postmenopausal women. Maturitas. 2002;41:35-43.

155. Rymer J, Morris EP. Extracts from "Clinical evidence": Menopausal symptoms. BMJ. 2000;321:1516-9.

156. Modelska K, Cummings S. Tibolone for postmenopausal women: systematic review of randomized trials. J Clin Endocrinol Metab. 2002;87:16-23.

157. Baracat EC, Barbosa IC, Giordano MG, Haidar MA, Marinho RM, Menegocci JC, et al. A randomized, open-label study of conjugated equine estrogens plus medroxyprogesterone acetate versus tibolone: effects on symptom control, bleeding pattern, lipid profile and tolerability.Climacteric. 2002 Mar;5(1):60-9.

158. Formoso G, Perrone E, Maltoni S, Bladuzzi S, D’Amico R, Bassi C, Basevi V, Marata AM, Magrini N, Maestri E. Short and long term effectos of tibolone in postmenopausal women (Review). Cochrane Database of Systematic Reviews 2012, Issue 2. Art. No.: CD 0085.

159. Bouchard P, Panay N, de Villiers TJ, Vincendon P, Bao W, Cheng RF, Constantine G. Randomized placebo- and active-controlled study of desvenlafaxine for menopausal vasomotor symptoms. Climacteric. 2012;15:12-20.

160. Beral V. Million Women Study Collaborators. Breast cancer and hormonereplacement therapy in the Million Women Study. Lancet. 2003;362:419-27.

161. Shams T, Firwana B, Habib F, Alshahrani A, Alnouh B, Murad MH, Ferwana M. SSRIs for Hot Flashes: A Systematic Review and MetaAnalysis of Randomized Trials. J Gen Intern Med. 2013 Jul 26 [Epub ahead or print]. doi:10.1007/s11606-013-2535-9. PubMed. 
162. Pinkerton JV, Constantine G, Hwang E, Cheng RF. Desvenlafaxine compared with placebo for treatment of menopausal vasomotor symptoms: A 12-week, multicenter, parallel-group, randomized, double-blind, placebocontrolled efficacy trial. Menopause. 2013;20:28-37.

163. Pinkerton JV, Archer DF, Guico-Pabia CJ, Hwang E, Cheng RF. Maintenance of the efficacy of desvenlafaxine in menopausal vasomotor symptoms: a 1-year randomized controlled trial. Menopause. 2013;20:38-46.

164. Archer DF, Pinkerton JV, Guico-Pabia CJ, Hwang E, Cheng RF; Study 3353 Investigators. Cardiovascular, cerebrovascular, and hepatic safety of desvenlafaxine for 1 year in women with vasomotor symptoms associated with menopause. Menopause. 2013;20:47-56.

165. Nelson HD, Vesco KK, Haney E, Fu R, Nedrow A, Miller J et al. Nonhormonal therapies for menopausal hot flashes: systematic review and meta-analysis. JAMA. 2006;295:2057-71.

166. Soares CN, Joffe H, Viguera AC, Petrillo L, Rydzewski M, Yehezkel R et al. Paroxetine versus placebo for women in midlife after hormone therapy discontinuation. Am J Med. 2008;121:159-62.

167. Stearns V, Beebe KL, Iyengar M, Dube E. Paroxetine controlled release in the treatment of menopausal hot flashes: a randomized controlled trial. JAMA. 2003;289:2827-34.

168. Stearns V, Slack R, Gree N, et al. Paroxetine is an effective treatment for hot flashes: results form a prospective randomized clinical trial. J Clin Oncol. 2005;23:6919-30.

169. Kelly CM, Juurlink DN, Gomes T, Duong-Hua M, Pritchard KI, Austin PC et al. Selective serotonin reuptake inhibitors and breast cancer mortality in women receiving tamoxifen: a population based cohort study. BMJ. 2010;340:c693.

170. Simon JA, Portman DJ, Kaunitz AM, Mekonnen H, Kazempour K, Bhaskar S, et al. Low-dose paroxetine $7.5 \mathrm{mg}$ for menopausal vasomotor symptoms: two randomized controlled trials. Menopause [Internet]. 2013 Oct [cited 2014 Oct 24];20(10):1027-35. Available from: http://www.ncbi.nlm.nih.gov/pubmed/ 24045678

171. Suvanto-Luukkonen E, Koivunen R, Sundstrom H, Bloigu R, Karjalainen E, Haiva-Mallinen $\mathrm{L}$ et al. Citalopram and fluoxetine in the treatment of postmenopausal symptoms: a prospective, randomized, 9-month, placebocontrolled, double-blind study. Menopause. 2005;12:18-26.

172. Loprinzi CL, Sloan JA, Perez EA, Quella SK, Stella PJ, Mailliard JA et al. Phase III Evaluation of Fluoxetine for treatment of hot flashes. J Clin Oncol. 2002;20:1578-83. 
173. Loprinzi CL, Sloan J, Stearns V, Slack R, Iyengar M, Diekmann B et al. Newer antidepressants and gabapentin for hot flashes: an individual patient pooled analysis. J Clin Oncol. 2009;27:2831-7.

174. Wu M F, Hilsenbeck SG, Tham YL, Kramer R, Elledge RM, Chang J et al. The efficacy of sertraline for controlling hot flashes in women with or at high risk of developing breast cancer. Breast Cancer Res Treat. 2009; 118:369-75.

175. Grady D, Cohen B, Tice J, Kristof M, Olyaie A, Sawaya GF. Ineffectiveness of sertraline for treatment of menopausal hot flushes. Randomized controlled trial. The American College of Obstetricians and Gynecologists. 2007;109:823-30.

176. Gordon PR, Kerwin JP, Green-Boesen K, Senf J. Sertraline to treat hot flashes: a randomized controlled, double-blind, crossover trial in a general population. Menopause. 2006;13:568-75.

177. Buijs C, Mom CH, Willemse PHB, Boezen HM, Maurer JM, Wymenga ANM et al. Venlafaxine versus clonidine for the treatment of hot flashes in breast cancer patients: a double-blind, randomized cross-over study. Breast Cancer Res Treat. 2009;115:573-80.

178. Loibl S, Schwedler k, von Minckwitz G, Strohmeier R, Mehta KM, Kaufmann M. Venlafaxine is superior to clonidine as treatment of hot flashes in breast cancer patients. A double blind randomized study. Annals of Oncology. 2007;18:689-93.

179. Carpenter JS, Storniolo AM, Johns S, Monahan PO, Azzouz F, Elam JL et al. Randomized, double-blind, placebo-controlled crossover trials of venlafaxine for hot flashes after breast cancer. Oncologist 2007;12:124-35.

180. Loprinzi CL, Kugler JW, Sloan JA, Mailliard JA, LaVasseur BI, Barton DL et al. Venlafaxine in management of hot flashes in survivors of breast cancer: a randomised controlled trial. The Lancet. 2000;356:2059-63.

181. Buijs C, Mom CH, Willemse PHB, Boezen HM, Maurer JM, Wymenga ANM et al. Venlafaxine versus clonidine for the treatment of hot flashes in breast cancer patients: a double-blind, randomized cross-over study. Breast Cancer Res Treat. 2009;115:573-80.

182. Sun Z, Hao Y, Zhang M. Efficacy and safety of desvenlafaxine treatment for hot flashes associated with menopause: a meta-analysis of randomized controlled trials. Gynecol Obstet Invest [Internet]. 2013 Jan [cited 2014 Oct 24];75(4):25562. Available from: http://www.ncbi.nlm.nih.gov/pubmed/23548358

183. Barton DL, Lavasseur BI, Sloan JA, Stawis AN, Flynn KA, Dyar M, et al. Phase III, Placebo-Controlled Trial of Three Doses of Citalopram for the Treatment of Hot Flashes: NCCTG Trial N05C9. J Clin Oncol. 2010; 28:3278-83. 
184. Aguirre W, Chedraui P, Mendoza J, Ruilova I. Gabapentin vs. low-dose transdermal estradiol for treating post-menopausal women with moderate to very severe hot flushes. Gynecol Endocrinol. 2010;26:333-7.

185. Patorno E, Bohn RL, Wahl PM, Avorn J, Patrick AR, Liu J et al. Anticonvulsant medications and the risk of suicide, attempted suicide, or violent death. JAMA. 2010;303:1401-9.

186. Loprinzi CL, Kugler JW, Barton DL, Dueck AC, Tschetter LK, Nelimark RA et al. Phase III Trial of Gabapentin Alone or in Conjunction With an Antidepressant in the Management of Hot Flashes in Women Who Have Inadequate Control With an antidepressant alone: NCCTG N03C5. J Clin Oncol. 2007 Jan 20;25:308-12.

187. Nesheim BI, Saetre T. Reduction of menopausal hot flashes by methyldopa: a double-blind crossover trial. Eur J Clin Pharmacol. 1981;20:413-6.

188. Hammond MG, Hatley L, Talbert LM. A double blind study to evaluate the effect of methyldopa on menopausal vasomotor flushes. J Clin Endocrinol Metab. 1983; 58:1158-60.

189. Andersen O, Engebretsen T, Solberg V M, Orbo A. Alpha-methyldopa for climacteric hot flushes. Acta Obstet Gynecol Scand. 1986;65:405-9.

190. Butt DAM, Lock MMCF, Lewis JEMMF, Ross SBMPM, Moineddin RP. Gabapentin for the treatment of menopausal hot flashes: a randomized controlled trial. Menopause. 2008;15:310-8.

191. Kimmick GG, Lovato J, McQuellon R, Robinson E, Muss HB. Randomized, double-blind, placebo-controlled, crossover study of sertraline (Zoloft) for the treatment of hot flashes in women with early stage breast cancer taking tamoxifen. The Breast J. 2006;12:114-22.

192. Reddy SYM, Warner HM, Guttuso TJM, Messing SM, DiGrazio WM, Thornburg LM et al. Gabapentin, Estrogen, and Placebo for Treating Hot Flushes: A Randomized Controlled Trial. Obstet Gynecol. 2006;108:41-8.

193. Guttuso T, Kurlan R, McDermott MP, Kieburtz K. Gabapentin's effects on hot flashes in postmenopausal women: a randomized controlled trial. Obstetrics \& Gynecology. 2003;101:337-45.

194. Pandya KJ, Morrow GR, Roscoe JA, Zhao H, Hickok JT, Pajon E et al. Gabapentin for hot flashes in 420 women with breast cancer: a randomised double-blind placebo-controlled trial. The Lancet. 2005;366:818-24.

195. Knight DC, Eden JA. A review of the clinical effects of phytoestrogens. Obstet Gynecol [Internet]. 1996 May [cited 2014 Sep 23];87(5 Pt 2):897-904. Available from: http://www.ncbi.nlm.nih.gov/pubmed/8677131

196. Melby MK, Lock M, Kaufert P. Culture and symptom reporting at menopause. Hum Reprod Update [Internet]. [cited 2014 Sep 15];11(5):495512. Available from: http://www.ncbi.nlm.nih.gov/pubmed/15919681 
197. Nedrow A, Miller J, Walker M, Nygren P, Huffman LH, Nelson HD. Complementary and alternative therapies for the management of menopauserelated symptoms: a systematic evidence review. Arch Intern Med [Internet]. 2006 Jul 24 [cited 2014 Sep 23];166(14):1453-65. Available from: http://www.ncbi.nlm.nih.gov/pubmed/16864755

198. Bolaños R, Del Castillo A, Francia J. Soy isoflavones versus placebo in the treatment of climacteric vasomotor symptoms: Systematic review and metaanalysis. Menopause. 2010. 17: 660-6.

199. Taku K, Melby MK, Kronenberg F, Kurzer MS, Messina M. et al. Extracted or synthesized soybean isoflavones reduce menopausal hot flash frequency and severity: systematic review and meta-analysis of randomized controlled trials. Menopause. 2012;19:776-90.

200. Bolaños-Díaz R, Zavala-Gonzales JC, Mezones-Holguín E, Francia-Romero J. Soy extracts versus hormone therapy for reduction of menopausal hot flushes: Indirect comparison. Menopause. 2011; 18: 825-9.

201. Lipovac M, Chedraui P, Gruenhut C, Gocan A, Kurz C, Neuber B, et al. The effect of red clover isoflavone supplementation over vasomotor and menopausal symptoms in postmenopausal women. Gynecol Endocrinol. 2012; 28: 203-7.[Incluído en T].

202. Ciaci A, Cicero AFG, Colacurci N, Matarazzo MG, De Leo V. Activity of isoflavones and berberine on vasomotor symptoms and lipid profile in menopausal women. Gynecol Endocrinol. 2012; 28: 699-702.

203. Aso T, Uchiyama S, Matsumura Y, Taguchi M, Nozaki M, Takamatsu K, et al. A natural S-equol supplement alleviates hot flushes and other menopausal symptoms in equol nonproducing postmenopausal Japanese women. J Women's Health. 2012;21:92-100.

204. Ye YB, Wang Z-L, Zhuo S-Y, Lu W, Liao H-F, Verbruggen M, et al. Soy germ isoflavones improve menopausal symptoms but have no effect on blood lipids in early postmenopausal Chinese women: A randomized placebo-controlled trial. Menopause. 2012;19:791-8.

205. Jou HJ, Wu SC, Chang FW, Ling PY, Chu KS, Wu WH. Effect of intestinal production of equol on menopausal symptoms in women treated with soy isoflavones. Int J Gynecol Obstetr 2008;102: 44-9.

206. Colli MC, Bracht A, Soares AA, de Oliveira AL, Boer CG, de Souza CGM, et al. Evaluation of the efficacy of flaxseed meal and flaxseed extract in reducing menopausal symptoms. J Med Food 2012; 15: 840-5.

207. Hanachi P, Golkho S. Assessment of soy phytoestrogens and exercise on lipid profiles and menopause symptoms in menopausal women. J Biological Sci 2008;8:789-93. 
208. Ferrari A. Soy extract phytoestrogens with high dose of isoflavones for menopausal symptoms. Journal of Obstetric and Gynaecological Research 2009;35:1083-90.

209. Del Giorno C, Maggio da Fonseca A, Bagnoli VR, Serrano de Assis J, Soares $\mathrm{JM}$, Baracat EC. Effects of trifolium pratense on climacteric and sexual symptoms in postmenopausal women. Revista da Associacao Medica Brasileira 2010;56:558-62.

210. Bicca MN, De O,Horta BL, Lethaby AE. Double-blind ranbdomized clinical trial to assess the effectiveness of soy isoflavones in the relieve of climateric symptoms (Unpublished study).

211. Campagnoli C, Abbà C, Ambroggio S, Peris C, Perona M, Sanseverino P. Polyunsaturated fatty acids (PUFAs) might reduce hot flushes: an indication from two controlled trials on soy isoflavones alone and with a PUFA supplement. Maturitas [Internet]. 2005 Jun 16 [cited 2014 Sep 24];51(2):127-34. Available from: http://www.sciencedirect.com/science/article/ pii/ S0378512204003627

212. Han KK, Soares JM, Haidar MA, de Lima GR, Baracat EC. Benefits of soy isoflavone therapeutic regimen on menopausal symptoms. Obstet Gynecol [Internet]. 2002 Mar [cited 2014 Sep 24];99(3):389-94. Available from: http://www.ncbi.nlm.nih.gov/pubmed/11864664

213. Khaodhiar L, Ricciotti HA, Li L, Pan W, Schickel M, Zhou J, et al. Daidzein-rich isoflavone aglycones are potentially effective in reducing hot flashes in menopausal women. Menopause [Internet]. [cited 2014 Sep 24];15(1):125-32. Available from: http://www.pubmedcentral.nih.gov/articlerender.fcgi? artid $=3207039 \&$ tool $=$ pmce ntrez\&rendertype $=$ abstract

214. Penotti M, Fabio E, Modena AB, Rinaldi M, Omodei U, Viganó P. Effect of soy-derived isoflavones on hot flushes, endometrial thickness, and the pulsatility index of the uterine and cerebral arteries. Fertil Steril [Internet]. 2003 May [cited 2014 Sep 24];79(5):1112-7. Available from: http://www.ncbi.nlm.nih.gov/pubmed/12738504

215. Upmalis DH, Lobo R, Bradley L, Warren M, Cone FL, Lamia CA. Vasomotor symptom relief by soy isoflavone extract tablets in postmenopausal women: a multicenter, double-blind, randomized, placebocontrolled study. Menopause [Internet]. [cited 2014 Sep 24];7(4):236-42. Available from: http://www.ncbi.nlm.nih.gov/pubmed/10914616

216. Nahas EAP, Nahas-Neto J, Orsatti FL, Carvalho EP, Oliveira MLCS, Dias R. Efficacy and safety of a soy isoflavone extract in postmenopausal women: a randomized, double-blind, and placebo-controlled study. Maturitas [Internet]. 2007 Nov 20 [cited 2014 Sep 23];58(3):249-58. Available from: http://www.ncbi.nlm.nih.gov/pubmed/17913408 
217. Levis S, Strickman-Stein N, Ganjei-Azar P, Xu P, Doerge D, Krischer J. Soy isoflavones in the prevention of menopausal bone loss and menopausal symptoms. Arch Intern Med 2011;171(15):1363-8.

218. Faure ED, Chantre P, Mares P. Effects of a standardized soy extract on hot flushes: a multicenter, double-blind, randomized, placebo-controlled study. Menopause. 9(5):329-34.

219. Kaari C, Haidar MA, Júnior JMS, Nunes MG, Quadros LG de A, Kemp C, et al. Randomized clinical trial comparing conjugated equine estrogens and isoflavones in postmenopausal women: a pilot study. Maturitas [Internet]. 2006 Jan 10 [cited 2014 Sep 26];53(1):49-58. Available from: http://www.ncbi.nlm.nih.gov/pubmed/16257151

220. Evans M, Ellito JG, Sharma P, Berman R, Guthrie N. The effect of synthetic genistein on menopause symptom management in healthy postmenopausal women: a multicenter, randomised, placebo-controlled study. Maturitas 2011;68:189-96.

221. Crisafulli A, Marini H, Bitto A, Altavilla D, Squadrito G, Romeo A, et al. Effects of genistein on hot flushes in early postmenopausal women: a randomized, double-blind EPT- and placebo-controlled study. Menopause [Internet]. [cited 2014 Sep 15];11(4):400-4. Available from: http://www.ncbi.nlm.nih.gov/pubmed/15243277

222. D'Anna R, Cannata ML, Marini H, Atteritano M, Cancellieri F, Corrado F, et al. Effects of the phytoestrogen genistein on hot flushes, endometrium, and vaginal epithelium in postmenopausal women: a 2-year randomized, doubleblind, placebo-controlled study. Menopause [Internet]. [cited 2014 Sep 26];16(2):301-6. Available from: http://www.ncbi.nlm.nih.gov/pubmed/19034051

223. Agosta $\mathrm{C}$, Atlante $\mathrm{M}$, Benvenuti C. Randomized controlled study on clinical efficacy of isoflavones plus Lactobacillus sporogenes, associated or not with a natural anxiolytic agent in menopause. Minerva Ginecol [Internet]. 2011 Mar [cited 2014 Sep 29];63(1):11-7. Available from: http://www.ncbi.nlm.nih.gov/pubmed/21311416

224. Cheng G, Wilczek B, Warner M, Gustafsson J-A, Landgren B-M. Isoflavone treatment for acute menopausal symptoms. Menopause [Internet]. [cited 2014 Sep 26];14(3 Pt 1):468-73. Available from: http://www.ncbi.nlm.nih.gov/pubmed/17290160

225. Albertazzi P, Pansini F, Bonaccorsi G, Zanotti L, Forini E, De Aloysio D. The effect of dietary soy supplementation on hot flushes. Obstet Gynecol [Internet]. 1998 Jan [cited 2014 Sep 29];91(1):6-11. Available from: http://www.ncbi.nlm.nih.gov/pubmed/9464712

226. Brzezinski A, AdlercreutzH, Shaoul R, Rosler A, Shmueli A, Tanos V, Schenker JG. Short-term effects of phytoestrogen-rich diet on postmenopuasal women. Menopause: The Journal of the North American Menopuase Society. 1997;4:89-94. 
227. Cheng G, Wilczek B, Warner M, Gustafsson J-A, Landgren B-M. Isoflavone treatment for acute menopausal symptoms. Menopause [Internet]. [cited 2014 Sep 26];14(3 Pt 1):468-73. Available from: http://www.ncbi.nlm.nih.gov/ pubmed/17290160

228. Carmigiani LO, Pedro AO, Cost-Paiva LH, Pinto-Neto AM. The effect of dietary soy supplementation compared to estrogen and placebo on menopausal symptoms: a randomised controlled trial. Maturitas 2010;67:262-9.

229. Radhakrishnan G, Agarwal N, Vaid N. Evaluation of isoflavone rich soy protein supplementation for postmenopausal therapy. Pakistan Journal of Nutrition. 2009;8:1009-17. Radhakrishnan G, Agarwal N, Vaid N. Evaluation of isoflavone rich soy protein supple.

230. Balk JL, Whiteside DA, Naus G, DeFerrari E, Roberts JM. A pilot study of the effects of phytoestrogen supplementation on postmenopausal endometrium. J Soc Gynecol Investig [Internet]. [cited 2014 Sep 29];9(4):23842. Available from: http://www.ncbi.nlm.nih.gov/pubmed/12113884

231. Burke GL, Legault C, Anthony M, Bland DR, Morgan TM, Naughton MJ, et al. Soy protein and isoflavone effects on vasomotor symptoms in periand postmenopausal women: the Soy Estrogen Alternative Study. Menopause [Internet]. [cited 2014 Sep 29];10(2):147-53. Available from: http://www.ncbi.nlm.nih.gov/pubmed/12627040

232. Dalais FS, Rice GE, Wahlqvist ML, Grehan M, Murkies AL, Medley G, et al. Effects of dietary phytoestrogens in postmenopausal women. Climacteric [Internet]. 1998 Jun [cited 2014 Sep 29];1(2):124-9. Available from: http://www.ncbi.nlm.nih.gov/pubmed/11907915

233. Knight DC, Howes JB, Eden JA, Howes LG. Effects on menopausal symptoms and acceptability of isoflavone-containing soy powder dietary supplementation. Climacteric [Internet]. 2001 Mar [cited 2014 Sep 29];4(1):13-8. Available from: http://www.ncbi.nlm.nih.gov/pubmed/11379373

234. Kotsopoulos D, Dalais FS, Liang YL, McGrath BP, Teede HJ. The effects of soy protein containing phytoestrogens on menopausal symptoms in postmenopausal women. Climacteric [Internet]. 2000 Sep [cited 2014 Sep 29];3(3):161-7. Available from: http://www.ncbi.nlm.nih.gov/pubmed/11910617

235. Lewis JE, Nickell LA, Thompson LU, Szalai JP, Kiss A, Hilditch JR. A randomized controlled trial of the effect of dietary soy and flaxseed muffins on quality of life and hot flashes during menopause. Menopause [Internet]. [cited 2014 Sep 22];13(4):631-42. Available from: http://www.ncbi.nlm.nih.gov/pubmed/16837885

236. St Germain A, Peterson CT, Robinson JG, Alekel DL. Isoflavone-rich or isoflavone-poor soy protein does not reduce menopausal symptoms during 24 weeks of treatment. Menopause [Internet]. [cited 2014 Sep 16];8(1):1726. Available from: http://www.ncbi.nlm.nih.gov/pubmed/11201510 
237. Heyerick A, Vervarcke S, Depypere H, Bracke M, De Keukeleire D. A first prospective, randomized, double-blind, placebo-controlled study on the use of a standardized hop extract to alleviate menopausal discomforts. Maturitas [Internet]. 2006 May 20 [cited 2014 Sep 29];54(2):164-75. Available from: http://www.ncbi.nlm.nih.gov/pubmed/16321485

238. Heger M, Ventskovskiy BM, Borzenko I, Kneis KC, Rettenberger R, Kaszkin-Bettag M, et al. Efficacy and safety of a special extract of Rheum rhaponticum (ERr 731) in perimenopausal women with climacteric complaints: a 12-week randomized, double-blind, placebo-controlled trial. Menopause [Internet]. [cited 2014 Sep 29];13(5):744-59. Available from: http://www.ncbi.nlm.nih.gov/pubmed/16894335

239. Tempfer CB, Froese G, Heinze G, Bentz E-K, Hefler LA, Huber JC. Side effects of phytoestrogens: a meta-analysis of randomized trials. Am J Med [Internet]. 2009 Oct [cited 2014 Sep 25];122(10):939-46.e9. Available from: http://www.ncbi.nlm.nih.gov/ pubmed/19786161

240. Velentzis LS, Cantwell MM, Cardwell C, Keshtgar MR, Leathem AJ, Woodside $\mathrm{J}$ V. Lignans and breast cancer risk in pre- and post-menopausal women: metaanalyses of observational studies. Br J Cancer [Internet]. 2009 May 5 [cited 2014 Sep 29];100(9):1492-8. Available from: http://www.pubmedcentral.nih.gov/ articlerender.fcgi artid $=2694431 \&$ tool $=$ pmcentrez\&rendertype $=$ abstract

241. Leach MJ, Moore V. Black cohosh (Cimicifuga spp.) for menopausal symptoms. Cochrane Database of Systematic Reviews. 2012, issue 9. Art. No.: CD007244. DOI: 10.1002/14651858.CD007244.pub2.

242. Laakmann E, Grajecki D, Doege K, zu Eulenburg C, Buhling KJ. Efficacy of Cimifuga Racemosa, Hypericum perforatum and Agnus castus in the treatment of climacteric complaints: a systematic review. Gynecol Endocrinol. 2012; 28: 703-9.

243. Naser B, Schnitker J, Minkin MJ, de Arriba SG, Nolte K-U, Osmers R. Suspected black cohosh hepatotoxicity: no evidence by meta-analysis of randomized controlled clinical trials for isopropanolic black cohosh extract. Menopause. 2011; 18: 366-75.

244. Ross SM. Menopause: a standardized isopropanolic black cohosh extract (remifemin) is found to be safe and effective for menopausal symptoms. Holist Nurs Pract. 2012; 26: 58-61.

245. Lee MS, Shin B-C, Yang EJ, Lim H-J, Ernst E. Maca (Lepidium meyenii) for treatment of menopausal symptoms: A systematic review. Maturitas. 2011; 79: 227-33.

246. Kim SY, Seo SK, Choi YM, Jeon YE, Lim KJ, Cho S, et al. Effects of red ginseng supplementation on menopausal symptoms and cardiovascular risk factors in postmenopausal women: A double-blind randomized controlled trial. Menopause. 2012;19:461-6. 
247. Nahidi F, Kariman N, Simbar M, Mojab F. The study on the effects of Pimpinella anisum on relief and recurrence of menopausal hot flashes. Iranian J Pharmaceutical Res. 2012;11:1079-85.

248. Nahidi F, Zare E, Mojab F, Alavi-Majd H. Effects of licorice on relief and recurrence of menopausal hot flashes. Iranian J Pharmaceutical Res.2012; 11:541-8.

249. Mirabi P, Mojab F. The effects of valerian root on hot flashes in menopausal women. Iranian J Pharmaceutical Res. 2013;12:217-22.

250. Van Die MD, Burger HG, Bone KM, Cohen MM, Teede HJ. Hypericum perforatum with Vitex agnus-castus in menopausal symptoms: a randomized, controlled trial. Menopause [Internet]. [cited 2014 Sep 29];16(1):156-63. Available from: http://www.ncbi.nlm.nih.gov/pubmed/18791483

251. Abdali K, Khajehei M, Tabatabaee HR. Effect of St John's wort on severity, frequency, and duration of hot flashes in premenopausal, perimenopausal and postmenopausal women: a randomized, double-blind, placebo-controlled study. Menopause [Internet]. 2010 Mar [cited 2014 Sep 29];17(2):326-31. Available from: http://www.ncbi.nlm.nih.gov/pubmed/20216274

252. Uebelhack R, Blohmer J-U, Graubaum H-J, Busch R, Gruenwald J, Wernecke K-D. Black cohosh and St. John's wort for climacteric complaints: a randomized trial. Obstet Gynecol [Internet]. 2006 Mar [cited 2014 Sep 29];107(2 Pt 1):247-55. Available from: http://www.ncbi.nlm.nih.gov/pubmed/16449108

253. Ernst E. Second thoughts about safety of St John's wort. Lancet [Internet]. 1999 Dec 11 [cited 2014 Sep 29];354(9195):2014-6. Available from: http://www.ncbi.nlm.nih.gov/pubmed/10636361

254. Hammerness P, Basch E, Ulbricht C, Barrette E-P, Foppa I, Basch S, et al. St John's wort: a systematic review of adverse effects and drug interactions for the consultation psychiatrist. Psychosomatics [Internet]. [cited 2014 Sep 29];44(4):271-82. Available from: http://www.ncbi.nlm.nih.gov/pubmed/12832592

255. Knüppel L, Linde K. Adverse effects of St. John's Wort: a systematic review. J Clin Psychiatry [Internet]. 2004 Nov [cited 2014 Sep 29];65(11):1470-9. Available from: http://www.ncbi.nlm.nih.gov/pubmed/15554758

256. Whitten DL, Myers SP, Hawrelak JA, Wohlmuth H. The effect of St John's wort extracts on CYP3A: a systematic review of prospective clinical trials. $\mathrm{Br} \mathrm{J}$ Clin Pharmacol [Internet]. 2006 Nov [cited 2014 Sep 29];62(5):512-26. Available from: http://www.pubmedcentral.nih.gov/articlerender.fcgi? artid $=1885170 \&$ tool $=$ pmce ntrez\&rendertype $=$ abstract

257. Brooks NA, Wilcox G, Walker KZ, Ashton JF, Cox MB, Stojanovska L. Beneficial effects of Lepidium meyenii (maca) on psychological symptoms and measures of sexual dysfunction in postmenopausal women are not related to estrogen or androgen content. Menopause. 2008;15:1157-62. 
258. Meissner HO, Reich-Bilinska H, Mscisz A, Kedzia B. Therapeutic effects of pre-gelatinized organic maca (Lepidium peruvanum chacon) used as a nonhormonal alternative to HRT in perimenopausal women-clinical pilot study. Int J Biomed Sci 2006;2:143-59.

259. Meissner HO, Mscisz A, Reich-Bilinska H, Kapczynski W, Mrozikiewicz P, Bobkiewicz-Kozlowska T, Kedzia B, Lowicka A, Barchia I. HormoneBalancing Effect of Pre-Gelatinized Organic Maca (Lepidium peruvianum Chacon): (II) Physiological and Symptomatic Responses of EarlyPostmenopausal Women to Standardized doses of Maca in Double Blind, Randomized, Placebo-Controlled, Multi-Centre Clinical Study. Int J Biomed Sci. 2006 Dec;2(4):360-74.

260. Chen HY, Cho WCS, Sze SCW, Tong Y. Treatment of menopausal symptoms with Er-xian decoction: a systematic review. Am J Chin Med [Internet]. 2008 Jan [cited 2014 Sep 29];36(2):233-44. Available from: http://www.ncbi.nlm.nih.gov/pubmed/18457358

261. Jeri A.The Use of an Isoflavone Supplement to Relieve Hot Flushes [Internet]. [cited 2014 Sep 29]. Available from: http://promensil.ca/ en/hcp/PDF/jeriPromensil.pdf

262. Tice JA, Ettinger B, Ensrud K, Wallace R, Blackwell T, Cummings SR. Phytoestrogen supplements for the treatment of hot flashes: the Isoflavone Clover Extract (ICE) Study: a randomized controlled trial. JAMA [Internet]. 2003 Jul 9 [cited 2014 Sep 29];290(2):207-14. Available from: http://www.ncbi.nlm.nih.gov/pubmed/12851275

263. Agencia Española de Medicamentos y Productos Sanitarios [Internet]. Comunicación sobre riesgos de medicamentos para profesionales sanitarois; ref:2006/06 [citado 20 Jul 2006]. Nota Informativa: extracto de la raíz de "Cimifuga Racemosa" y lesiones hepáticas.

264. Al-Akoum M, Maunsell E, Verreault R, Provencher L, Otis H, Dodin S. Effects of Hypericum perforatum (St. John's wort) on hot flashes and quality of life in perimenopausal women: a randomized pilot trial. Menopause [Internet]. [cited 2014 Sep 29];16(2):307-14. Available from: http://www.ncbi.nlm.nih.gov/pubmed/19194342

265. Lee MS, Shin BC, Ernst E. Acupuncture for treating menopausal hot flushes: a systematic review. Climacteric. 2009;12:16-25.

266. Venzke L, Calvert JF Jr, Gilbertson B. A randomized trial of acupuncture for vasomotor symptoms in post-menopausal women. Complement Ther Med. 2010;18:59-66.

267. Sunay D, Ozdiken M, Arslan H, Seven A, Aral Y. The effect of acupuncture on postmenopausal symptoms and reproductive hormones: a sham controlled clinical trial. Acupunct Med. 2011;29:27-31. 
268. Painovich JM, Shufelt CL, Azziz R, Yang Y, Goodarzi MO, Braunstein GD, Karlan BY, Stewart PM, Merz CN. A pilot randomized, single-blind, placebo-controlled trial of traditional acupuncture for vasomotor symptoms and mechanistic pathways of menopause. Menopause. 2012;19:54-61.

269. Cho SH, Whang WW. Acupuncture for vasomotor menopausal symptoms: a systematic review. Menopause. 2009;16:1065-73.

270. Borud EK, Alraek T, White A, Fonnebo V, Eggen AE, Hammar M, Astrand LL, Theodorsson E, Grimsgaard S. The Acupuncture on Hot Flushes Among Menopausal Women (ACUFLASH) study, a randomized controlled trial. Menopause. 2009;16:484-93.

271. Park JE, Lee MS, Jung S et al. Moxibustion for treating menopausal hot flashes: a randomized clinical trial. Menopause. 2009;16:660-5.

272. O'Brien KA, Varigos E, Black C, Komesaroff PA. Laser acupuncture does not improve menopausal symptoms. Menopause. 2010;17:636-41.

273. Kim DI, Jeong JC, Kim KH, Rho JJ, Choi MS, Yoon SH, et al. Acupuncture for hot flushes in perimenopausal and postmenopausal women: a randomised, sham-controlled trial. Acupunct Med. 2011;29:249-56.

274. Zhou J, Qu F, Sang X, Wang X, Nan R. Acupuncture and auricular acupressure in relieving menopausal hot flashes of bilaterally ovariectomized chinese women: a randomized controlled trial. Evid Based Complement Alternat Med. 2011;2011:713274. doi: 10.1093/e.

275. Tremblay A, Sheeran L, Aranda SK. Psychoeducational interventions to alleviate hot flashes: a systematic review. Menopause. 2008;15:193-202.

276. Keefer L, Blanchard EB. A behavioral group treatment program for menopausal hot flashes: results of a pilot study. Appl Psychophysiol Biofeedback. 2005; 30:21-30.

277. Anarte MT, Cuadros JL, Herrera J. Hormonal and psychological treatment: therapeutic alternative for menopausal women? Maturitas. 1998;29:203-13.

278. Carmody JF, Crawford S, Salmoirago-Blotcher E, Leung K, Churchill L, Olendzki N. Mindfulness training for coping with hot flashes: results of a randomized trial. Menopause. 2011;18:611-20.

279. Innes KE, Selfe TK, Vishnu A. Mind-body therapies for menopausal symptoms: A systematic review. Maturitas. 2010;66:135-49.

280. Lindh-Åstrand L, Nedstrand E. Effects of applied relaxation on vasomotor symptoms in postmenopausal women: a randomized controlled trial. Menopause. 2013;20:401-8.

281. Irvin JH, Domar AD, Clark C, Zuttermeister PC, Friedman R. The effects of relaxation response training on menopausal symptoms. Journal of Psychosomatic Obstetrics and Gynaecology. 1996;17:202-7. 
282. Freedman RR, Woodward S. Behavioral treatment of menopausal hot flushes: evaluation by ambulatory monitoring. American Journal of Obstetrics and Gynecology. 1992;167:436-9.

283. Rankin M. Effect of low frequency sound on menopausal symptoms. Journal of Holistic Nursing. 1989;7:34-41.

284. Germaine LM, Freedman RR. Behavioral treatment of menopausal hot flashes: evaluation by objective methods. Journal of Consulting and Clinical Psychology.1984;52:1072-9.

285. Nedstrand E, Wijma K, Wyon Y, Hammar M. Applied relaxation and oral estradiol treatment of vasomotor symptoms in postmenopausal women. Maturitas. 2005;51:154-62.

286. Williamson J., White A., Hart A. \& Ernst E. Randomised controlled trial of reflexology for menopausal symptoms. BJOG. 2002;109:1050-5.

287. Hur MH, Yang YS, Lee MS. Aromatherapy massage affects menopausal symptoms in korean climacteric women: a pilot-controlled clinical trial. Evid Based Complement Alternat Med. 2008;5:325-8.

288. Ziaei S, Kazemnejad A, Zareai M. The effect of vitamin $\mathrm{E}$ on hot flashes in menopausal women. Gynecol Obstet Invest. 2007;64:204-7.

289. Lucas M, Asselin G, Merette C, Poulin MJ, Dodin S. Effects of ethyleicosapentaenoic acid omega-3 fatty acid supplementation on hot flashes and quality of life among middle-aged women: a double-blind, placebocontrolled, randomized clinical trial. Menopause. 2009;16:357-66.

290. Guttuso T, McDermott MP, Su H, Kieburtz K. Effects of L-isoleucine and L-valine on hot flushes and serum homocysteine: a randomized controlled trial. Obstet Gynecol. 2008;112:109-15.

291. Pandit S, Umbardand S, Ghodake V, Vats U, Tayade H, Rathod O. Evaluation Of The Efficacy And Tolerability Of Micronutrient Supplementation In Treatment Of Post Menopausal Symptoms. Intern J Genomics Proteomics. 2012; 6. [4 p]. Disponible en: http://isp.

292. Pastore LM, Carter RA, Hulka BS, Wells E. Self-reported urogenital symptoms in postmenopausal women: Women's Health Initiative. Maturitas. 2004;49:292-303.

293. Stenberg A, Heimer G, Ulmsten U, Cnattingius S. Prevalence of genitourinary and other climacteric symptoms in 61-year-old women. Maturitas. 1996;24:31-6.

294. Gold EB, Sternfeld B, Kelsey JL, Brown C, Mouton C, Reame N, et al. Relation of demographic and lifestyle factors to symptoms in a multiracial/ethnic population of women 40-55 years of age. Am J Epidemiol. 2000;152:463-73. 
295. Suckling JA,Kennedy R, Lethaby A, RobertsH. Local oestrogen for vaginal atrophy in postmenopausal women. Cochrane Database of Systematic Reviews 2006, Issue 4. Art. No.: CD001500. DOI: 10.1002/14651858.CD001500.pub2.

296. Galhardo CL, Soares JM Jr, Simoes RS, Haidar MA, Rodrigues de Lima G, Baracat EC. Estrogen effects on the vaginal $\mathrm{pH}$, flora and cytology in late postmenopause after a long period without hormone therapy. Clin Exp Obstet Gynecol. 2006; 33:85-9.

297. Simon JA, Reape KZ, Wininger S, Hait H. Randomized, multicenter, doubleblind, placebo-controlled trial to evaluate the efficacy and safety of synthetic conjugated estrogens B for the treatment of vulvovaginal atrophy in healthy postmenopausal women. Fertil Steril. 2008;90:1132-8. Epub 2007 Dec 3.

298. Freedman M, Kaunitz AM, Reape KZ, Hait H, Shu H. Twice-weekly synthetic conjugated estrogens vaginal cream for the treatment of vaginal atrophy. Menopause. 2009; 16:735-41.

299. Bachmann G, Lobo RA, Gut R, Nachtigall L, Notelovitz M. Efficacy of low-dose estradiol vaginal tablets in the treatment of atrophic vaginitis: a randomized controlled trial. Obstet Gynecol. 2008; 111:67-76.

300. Bachmann GA, Schaefers M, Uddin A, Utian WH. Microdose transdermal estrogen therapy for relief of vulvovaginal symptoms in postmenopausal women. Menopause. 2009; 16:877-82.

301. Cano A, Estévez J, Usandizaga R, Gallo JL, Guinot M, Delgado JL et al. The therapeutic effect of a new ultra low concentration estriol gel formulation ( $0.005 \%$ estriol vaginal gel) on symptoms and signs of postmenopausal vaginal atrophy: results from a pivotal phase III study. Menopause. 2012;19:1130-9.

302. Brunner RL, Aragaki A, Barnabei V, Cochrane BB, Gass M, Hendrix S, et al. Menopausal symptom experience before and after stopping estrogen therapy in the Women's Health Initiative randomized, placebo-controlled trial. Menopause. 2010;17:946-54.

303. Cummings SR, Ettinger B, Delmas PD, Kenemans P, Stathopoulos V, Verweij $\mathrm{P}$ et al. The effects of tibolone in older postmenopausal women. $\mathrm{N}$ Engl J Med. 2008;359:697-708.

304. Beral V. Million Women Study Collaborators. Breast cancer and hormonereplacement therapy in the Million Women Study. Lancet. 2003;362:419-427.

305. Kenemans P, Bundred NJ, Foidart JM, Kubista E, von Schoultz B, Sismondi $\mathrm{P}$ et al. Safety and efficacy of tibolone in breast-cancer patients with vasomotor symptoms: a double-blind, randomised, non-inferiority trial. Lancet Oncol. 2009;10:135-46.

306. Nedrow A, Miller J, Walker M, Nygren P, Huffman LH, Nelson HD. Complementary and alternative therapies for the management of menopauserelated symptoms. A systematic evidence review. Arch Intern med. 2006;166:1453-65. 
307. Howes LG, Howes JB, Knight DC. Isoflavone therapy for menopausal flushes: a systematic review and meta-analysis. Maturitas. 2006;55:203-11.

308. Hidalgo LA, Chedraui PA, Morocho N, Ross S, San Miguel G. The effect of red clover isoflavones on menopausal symptoms, lipids and vaginal cytology in menopausal women: a randomized, doubleblind, placebocontrolled study. Gynecol Endocrinol. 2005;21:257-64.

309. Manonai J, Songchitsomboon S, Chanda K, Hong JH, Komindr S. The effect of a soy-rich diet on urogenital atrophy: a randomized crossover trial. Maturitas. 2006;54:135-40.

310. Carmignani LO, Pedro AO, Costa-Paiva LH, Pinto-Neto AM. The effect of dietary soy supplementation compared to estrogen and placebo on menopausal symptoms: a randomized controlled trial. Maturitas. 2010;67:262-9.

311. Leach MJ, Moore V. Black cohosh (Cimicifuga spp.) for menopausal symptoms. Cochrane Database of Systematic Reviews. 2012, issue 9. Art. No.: CD007244. DOI: 10.1002/14651858.CD007244.pub2.

312. Laakmann E, Grajecki D, Doege K, zu Eulenburg C, Buhling KJ. Efficacy of Cimifuga Racemosa, Hypericum perforatum and Agnus castus in the treatment of climacteric complaints: a systematic review. Gynecol Endocrinol. 2012;28:703-9.

313. Reed SD, Newton KM, LaCroix AZ, Grothaus LC, Grieco VS, Ehrlich K. Vaginal, endometrial, and reproductive hormone findings: randomized, placebo-controlled trial of black cohosh, multibotanical herbs, and dietary soy for vasomotor symptoms: the Herbal Alternatives for Menopause (HALT) Study. Menopause. 2008;15:51-8.

314. Wuttke W, Seidlová-Wuttke D, Gorkow C. The Cimicifuga preparation BNO 1055 vs. conjugated estrogens in a double-blind placebo-controlled study: effects on menopause symptoms and bone markers. Maturitas. 2003;44:67-77.

315. Osmers R, Friede M, Liske E, Schnitker J, Freudenstein J, Henneicke-von Zepelin HH. Efficacy and safety of isopropanolic black cohosh extract for climacteric symptoms. Obstet Gynecol. 2005;105:1074-83.

316. Bachmann GA, Komi JO. Ospemifene effectively treats vulvovaginal atrophy in postmenopausal women: results from a pivotal phase 3 study. Menopause. 2010;17:480-6.

317. Simon JA, Lin VH, Radovich C, Bachmann GA; Ospemifene Study Group. One-year long-term safety extension study of ospemifene for the treatment of vulvar and vaginal atrophy in postmenopausal women with a uterus. Menopause. 2013;20:418-27.

318. Kagan R, Williams RS, Pan K, Mirkin S, Pickar JH. A randomized, placeboand active-controlled trial of bazedoxifene/conjugated estrogens for treatment of moderate to severe vulvar/vaginal atrophy in postmenopausal women. Menopause. 2010;17:281-9. 
319. Bachmann G, Bobula J, Mirkin S. Effects of bazedoxifene/conjugated estrogens on quality of life in postmenopausal women with symptoms of vulvar/vaginal atrophy. Climacteric. 2010;13:132-40.

320. Vardy MD, Lindsay R, Scotti RJ, Mikhail M, Richart RM, Nieves J, et al. Short-term urogenital effects of raloxifene, tamoxifen and estrogen. Am J Obstet Gynecol. 2003;189:81-8.

321. Pinkerton JV, Shifren JL, La Valleur J, Rosen A, Roesinger M, Siddhanti S. Influence of raloxifene on the efficacy of an estradiol-releasing ring for treating vaginal atrophy in postmenopausal women. Menopause. 2003;10:45-52.

322. Parsons A, Merritt D, Rosen A, Heath H 3rd, Siddhanti S, Plouffe L Jr. Effect of raloxifene on the response to conjugated estrogen vaginal cream or nonhormonal moisturizers in postmenopausal vaginal atrophy. Obstet Gynecol. 2003; 101:346-52.

323. Lufkin EG, Whitaker MD, Nickelsen T, Argueta R, Caplan RH, Knickerbocker RK, et al. Treatment of established postmenopausal osteoporosis with raloxifene: a randomized trial. J Bone Miner Resm. 1998; 13:1747-54.

324. Draper MW, Flowers DE, Huster WJ, Neild JA, Harper KD, Arnaud C. A controlled trial of raloxifene (LY139481) HCl: impact on bone turnover and serum lipid profile in healthy postmenopausal women. Journal of Bone and Mineral Research. 1996;11:835-42.

325. Bygdeman M, Swahn ML. Replens versus dienoestrol cream in the symptomatic treatment of vaginal atrophy in postmenopausal women. Maturitas. 1996; 23:259-63.

326. Nachtigall LE. Comparative study: Replens versus local estrogen in menopausal women. Fertil Steril. 1994; 61: 178-180.

327. Foxman B, Barlow R, D'Arcy H, Guillespie B, Sobel JD. Urinary tract infection: self reported incidence and associated costs. Annals of Epidemiology. 2000;10:509-15.

328. Raz R, Gennesin Y, Wasser J, Stoler Z, Rosenfeld S, Rottensterich E, et al. Recurrent urinary tract infections in postmenopausal women. Clinical Infectious Diseases. 2000;30:152-6.

329. Waetjen LE, Ye J, Feng WY, et al. Association between menopausal transition and the development of urinary incontinence. Obstet Gynecol. 2009;114:989-98.

330. Perrotta C, Aznar M, Mejia R, Albert X, Ng CW. Oestrogens for preventing recurrent urinary tract infection in postmenopausal women. Cochrane Database of Systematic Reviews 2008, Issue 2. Art. No.: CD005131. DOI: 10.1002/14651858.CD005131.pub2. 
331. Raz R, Stamm WE. A controlled trial of intravaginal estriol in postmenopausal women with recurrent urinary tract infections. New England Journal of Medicine 1993;329:753-6.

332. Eriksen B. A randomized, open, parallel-group study on the preventive effect of an estradiol-releasing vaginal ring (Estring) on recurrent urinary tract infections in postmenopausal women. American Journal of Obstetrics \& Gynecology 1999;180:1072-9.

333. Raz R, Colodner R, Rohana Y, Battino S, Rottensterich E,Wasser I, et al. Effectiveness of estriol-containing vaginal pessaries and nitrofurantoin macrocrystal therapy in the prevention of recurrent urinary tract infection in postmenopausal women. Clin Infect Dis. 2003;36:1362-8. Epub 2003 May 21.

334. $\mathrm{Xu} \mathrm{R}, \mathrm{Wu} \mathrm{Y}, \mathrm{Hu} \mathrm{Y}$. Prevention and treatment of recurrent urinary system infection with estrogen cream in postmenopausal women. Chung-Hua Fu Chan Ko Tsa Chih [Chinese Journal of Obstetrics \& Gynecology]. 2001;36:531-3.

335. Hendrix SL, Cochrane BB, Nygaard IE, Handa VL, Barnabei VM, Iglesia C, et al. Effects of estrogen with and without progestin on urinary incontinence. JAMA 2005;293:935-48. 




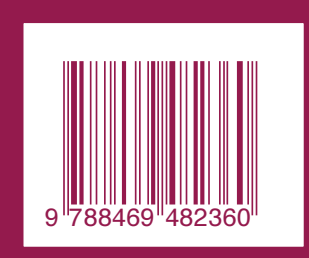

